\title{
Traveltime-Based True-Amplitude Migration
}

\author{
Dissertation \\ zur Erlangung des Doktorgrades \\ der Naturwissenschaften im Fachbereich \\ Geowissenschaften \\ der Universität Hamburg
}

\author{
vorgelegt von \\ Claudia Vanelle
}

aus

Hamburg

Hamburg

2002 
Als Dissertation angenommen vom Fachbereich Geowissenschaften der Universität Hamburg auf Grund der Gutachten von Prof. Dr. Dirk Gajewski,

Dr. Christian Hanitzsch

und Prof. Dr. Peter Hubral

Hamburg, den 3.April 2002

Prof. Dr. Ulrich Bismayer

Dekan

des Fachbereiches Geowissenschaften 
To my parents,

Heide and Gérard Vanelle. 

Don't forget the three virtues of a Geophysicist, namely:

\author{
True Love, \\ True Friendship, \\ True Amplitudes.
}

(Martin Tygel) 



\section{Abstract}

Amplitude-preserving migration based on a weighted diffraction stack is a task of high computational costs. Three factors determine the major contributions to the computational effort: The diffraction time surface must be computed for each image point as well as proper weight functions. Finally, the summation is carried out over the whole aperture of the experiment.

I propose a new strategy for amplitude-preserving migration that is entirely based on traveltimes, thus called traveltime-based true-amplitude migration. Its foundation is a hyperbolic traveltime expansion. High accuracy is achieved because second-order spatial derivatives are included in order to acknowledge the curvature of the wavefront. The algorithm permits the determination of the interpolation coefficients from traveltime tables sampled on a coarse grid, thus reducing the requirements in data storage. The method also provides a tool for the interpolation between sources. Application to various velocity models confirms that savings up to a factor of $10^{5}$ are possible in data storage with no significant loss in accuracy. Also, the interpolation is 5-6 times faster than the calculation of traveltime tables using a fast finite differences eikonal solver.

Since it is possible to express true-amplitude weight functions in terms of second-order traveltime derivatives a traveltime-based relationship for the weights can be established. As a consequence, the weight functions can be directly computed from the interpolation coefficients. This reduces the need in computational time, and particularly storage, because the weights are computed on-the-fly. Application of the weight functions shows good agreement between numerical and analytical results for the simple type of models considered in this work. For complex models the good accuracy of the geometrical spreading, which can be computed using the same interpolation coefficients as for the weight functions, was also demonstrated. This indicates that the migration technique will also perform well for complex models.

A further significant reduction of the computational effort involved in the migration can be achieved if the summation is carried out only over those traces that really contribute to the stack, i.e. by limiting the migration aperture. Moreover, the optimum migration aperture can also be determined from the traveltime coefficients. Examples show that limited aperture migration reduces the requirements in computational time by $80 \%$ in 2 $\mathrm{D}$, and by more than $90 \%$ in 3 -D media. At the same time, the image quality is enhanced by suppressing migration noise.

Since the foundation of the method, the hyperbolic traveltime expansion, is not limited to isotropic models the technique has a high potential to be extended to anisotropic media. 


\section{Contents}

1 Introduction 1

2 A Review of the Ray Method 5

2.1 The Elastodynamic Equation and Ray Series . . . . . . . . . . . . . . 5

2.2 The Eikonal Equations . . . . . . . . . . . . . . . . . . . . 7

2.3 The Transport Equations . . . . . . . . . . . . . . . . . . . . . . . . . . 9

2.4 Ray Tracing . . . . . . . . . . . . . . . . . . . . . . . . 12

2.5 Ray Centred Coordinates . . . . . . . . . . . . . . . . . . . . . 14

2.6 Propagator Matrices . . . . . . . . . . . . . . . . . . . . . . 18

2.7 Paraxial Traveltimes . . . . . . . . . . . . . . . . . . . 20

2.8 Interfaces . . . . . . . . . . . . . . . . . . . . . . . . 23

2.9 The Bortfeld Propagator . . . . . . . . . . . . . . . . . . . 27

3 Traveltime Interpolation $\quad 31$

3.1 Parabolic Traveltime Expansion . . . . . . . . . . . . . . . . . . . 32

3.2 Hyperbolic Traveltime Expansion . . . . . . . . . . . . . . . . . . 36

3.3 Accuracy of the Coefficients . . . . . . . . . . . . . . . . . 38

3.4 Examples . . . . . . . . . . . . . . . . . . . . . 45

3.5 Influence of the Grid spacing . . . . . . . . . . . . . . . . 55

3.6 Triplications . . . . . . . . . . . . . . . . 56

4 A $6 \times 6$ Propagator? $\quad 59$

4.1 The Matrix $\hat{\mathbf{I}} \ldots \ldots \ldots \ldots \ldots \ldots \ldots \ldots$

4.2 Curved Surfaces . . . . . . . . . . . . . . . . . . . . 60

4.3 Reflected Events . . . . . . . . . . . . . . . . . 63

5 Geometrical Spreading $\quad 65$

5.1 Introduction . . . . . . . . . . . . . . . . . 66 66

5.2 Spreading and Traveltime Derivatives . . . . . . . . . . . . 67

5.3 Examples ............................. 70

6 Amplitude Preserving Migration $\quad 77$

6.1 Introduction to Seismic Migration . . . . . . . . . . . . . . . 78

6.2 Derivation of True-Amplitude Weight Functions . . . . . . . . . . . . . . . 80

6.3 The Matrix $\underline{\mathrm{H}}_{\mathrm{F}} \ldots \ldots \ldots \ldots$. . . . . . . . . . . . . . . . . . . . . . 83

6.4 Geometrical Spreading and Final Weight Function . . . . . . . . . . . . 84 
6.5 Weight Functions for 2.5-D and 2-D . . . . . . . . . . . 86

6.6 Application to PP Data . . . . . . . . . . . . . . . . . . 88

6.7 Application to PS Converted Waves . . . . . . . . . . . . . . . . 94

6.8 Complex Models? . . . . . . . . . . . . . . . . . . 97

7 Limited Aperture Migration $\quad 103$

7.1 Optimum Migration Aperture . . . . . . . . . . . . . . . . . . . . 104

7.2 Examples . . . . . . . . . . . . . . . . . . . 106

7.3 Potential Savings in Computational Time . . . . . . . . . . . . . . 111

8 A General NMO Formula $\quad 113$

8.1 Introduction to Classical Formulae . . . . . . . . . . . . . . . . . . 114

8.2 The General NMO Formula . . . . . . . . . . . . . . . . . . . . . 114

8.3 The CRS Formula . . . . . . . . . . . . . . . . . . 117

9 Conclusions $\quad 121$

$\begin{array}{ll}10 \text { Outlook } & 123\end{array}$

10.1 Sensitivity to Velocity Errors . . . . . . . . . . . . . . . . . . . . 123

10.2 Migration with Angular Parametrisation . . . . . . . . . . . . . 125

10.3 Anisotropy . . . . . . . . . . . . . . . . . . 126

$\begin{array}{lr}\text { A Nomenclature } & 131\end{array}$

A.1 Indices, Vectors and Matrices _. . . . . . . . . . . . . . . . 131

A.2 List of Symbols . . . . . . . . . . . . . . . . . . . . . . . 131

A.2.1 Latin Letters . . . . . . . . . . . . . . . . . . . . . 132

A.2.2 Greek Letters . . . . . . . . . . . . . . . . . . . . . . . 134

B Measurement Configuration Matrices 135

$\begin{array}{ll}\text { C Analytic Coefficients } & 137\end{array}$

C.1 Constant Velocity Gradient Model . . . . . . . . . . . . . . . . . . . 137

C.2 Homogeneous Isotropic Model . . . . . . . . . . . . . . . . . . . . 138

C.3 Homogeneous Model with Elliptical Anisotropy . . . . . . . . . . . . . 140

$\begin{array}{ll}D \text { Coefficients } & 143\end{array}$

D.1 Parabolic Coefficients for Isotropic Media . . . . . . . . . . . . . . . . 144

D.2 Hyperbolic Coefficients for Isotropic Media . . . . . . . . . . . . . . 146

D.3 Coefficients for Anisotropic Media . . . . . . . . . . . . . . . . . . 148

E Reflector Independent Weight Functions $\quad 151$

$\begin{array}{ll}\text { Bibliography } & 157\end{array}$

$\begin{array}{ll}\text { Acknowledgements } & 163\end{array}$ 


\section{List of Figures}

2.1 Ray tube and geometrical spreading . . . . . . . . . . . . . . . . . 11

2.2 Behaviour of a ray tube at caustic points . . . . . . . . . . . . . . 13

2.3 Ray centred coordinates . . . . . . . . . . . . . . . . 15

2.4 Central and paraxial ray . . . . . . . . . . . . . . . . . 21

2.5 Interface coordinates . . . . . . . . . . . . . . . . . 24

2.6 Projection of the slowness into the reference plane . . . . . . . . . . . . 28

3.1 Determination of interpolation coefficients . . . . . . . . . . . . 34

3.2 Coefficients for a gradient model . . . . . . . . . . . . . . . . . 39

3.3 Errors of coefficients from analytic traveltimes . . . . . . . . . . . . . . 42

3.4 Errors of coefficients from numerical traveltimes . . . . . . . . . . . . . 43

3.5 Errors of numerical traveltimes . . . . . . . . . . . . . . . . . 44

3.6 Traveltime errors for a homogeneous model (analytic input) . . . . . . . . 46

3.7 Traveltime errors for a homogeneous model (Vidale input) . . . . . . . . . 47

3.8 Traveltime errors for a constant velocity gradient model (analytic input) . 49

3.9 Traveltime errors for a constant velocity gradient model (Vidale input) . . 50

3.10 The Marmousi velocity model . . . . . . . . . . . . . . . . . . . 52

3.11 Relative traveltime errors for the Marmousi model . . . . . . . . . . . . . 53

3.12 Absolute traveltime errors for the Marmousi model . . . . . . . . . . . . 54

3.13 Influence of the coarse grid on the accuracy . . . . . . . . . . . . 55

3.14 Triplication of a wavefront . . . . . . . . . . . . . . 56

3.15 Triplication of a traveltime curve . . . . . . . . . . . . . . . 57

3.16 Second derivatives and triplications in the Marmousi model . . . . . . . . 58

4.1 Coordinate systems of 2-D and 3-D propagators . . . . . . . . . . . 62

5.1 Geometrical spreading . . . . . . . . . . . . . . . . 66

5.2 Energy flux through a surface . . . . . . . . . . . . . . . 67

5.3 Angles in the Cartesian coordinate system . . . . . . . . . . . . . . . 68

5.4 Errors in geometrical spreading for a homogeneous model . . . . . . . . . . 72

5.5 Errors of Vidale traveltimes . . . . . . . . . . . . . . . . . . 73

5.6 Errors in geometrical spreading for a gradient model . . . . . . . . . . . . . 74

5.7 Comparison of spreading from two different algorithms . . . . . . . . . . 75

5.8 Geometrical spreading for the Marmousi model . . . . . . . . . . . . . . 75

6.1 Principle of Kirchhoff migration . . . . . . . . . . . . . . . . . . 79 
6.2 Superposition of point scatterers . . . . . . . . . . . . . . . . . 79

6.3 Common shot section for a horizontal reflector . . . . . . . . . . . . . . 89

6.4 Migrated depth section of a horizontal reflector . . . . . . . . . . . . . 89

6.5 Errors in reflection coefficients for a horizontal reflector . . . . . . . . . . . 91

6.6 Common shot section for an inclined reflector . . . . . . . . . . . . 92

6.7 Migrated depth section of an inclined reflector . . . . . . . . . . . . 92

6.8 Errors in reflection coefficients for an inclined reflector . . . . . . . . . . 93

6.9 Common shot section for a converted waves example . . . . . . . . . . 95

6.10 Migrated depth section for a converted wave example . . . . . . . . . . . 95

6.11 Recovered P-S reflection coefficients . . . . . . . . . . . . . . . . 96

6.12 Traveltimes for the PICROCOL model . . . . . . . . . . . . . . . . . . 98

6.13 Two-layer medium and substitute . . . . . . . . . . . . . . . . . 99

6.14 Weight function errors for a smoothed model . . . . . . . . . . . . . . 100

6.15 Two-layer medium and coarse grid . . . . . . . . . . . . . . . . 101

6.16 Weight function errors by interpolation . . . . . . . . . . . . . . 102

7.1 Diffraction and reflection traveltimes in a seismic section . . . . . . . . 105

7.2 Minimum migration aperture and Fresnel zone . . . . . . . . . . . 105

7.3 Synthetic section for a $63^{\circ}$ reflector . . . . . . . . . . . . . . . . 107

7.4 Recovered reflection coefficients for a $63^{\circ}$ reflector (noise-free) . . . . . . . 107

7.5 Exact and paraxial optimum migration aperture . . . . . . . . . . . . . 108

7.6 Recovered reflection coefficients for a horizontal reflector . . . . . . . . . . 109

7.7 Recovered reflection coefficients for a $63^{\circ}$ reflector (noised) . . . . . . . . . 109

7.8 Migrated depth sections for the $63^{\circ}$ reflector . . . . . . . . . . . . . . . 110

7.9 Potential savings in computational time . . . . . . . . . . . . . . . 112

10.1 Migration with angular parametrisation . . . . . . . . . . . . 125

10.2 Anisotropic traveltime interpolation errors . . . . . . . . . . . . . . 128

10.3 Geometrical spreading for an elliptical model . . . . . . . . . . . . . . . . . 129

D.1 Traveltimes designation on grid points . . . . . . . . . . . . . . . 144

E.1 Definition of ray centred coordinates . . . . . . . . . . . 153 


\section{List of Tables}

3.1 Coefficient errors for a constant velocity model . . . . . . . . . . . . . . . 40

3.2 Coefficient errors for a gradient model . . . . . . . . . . . . . . . . . 41

3.3 Traveltime errors for a homogeneous model . . . . . . . . . . . . . . . 48

3.4 Traveltime errors for a constant velocity gradient model . . . . . . . . . . . 51

3.5 Traveltime errors for a second constant velocity gradient model . . . . . . . 51

3.6 Traveltime errors for the Marmousi model . . . . . . . . . . . . . . . . . 52

5.1 Relative errors in geometrical spreading . . . . . . . . . . . . . 71 


\section{Chapter 1}

\section{Introduction}

The aim of this thesis is to provide an efficient strategy for the inversion of seismic reflection data. The motivation for a seismic experiment is to get information about the subsurface: what does it consist of, i.e. what are the material parameters, and how is it stratified. The result of the experiment is a seismic section, a time image of the subsurface. This image is, however, a distorted image that can not be directly translated into velocities and structure: the reflections present in the section do not show the correct positions and inclinations of the corresponding reflectors. A corrected map of the subsurface can be obtained by inverting the seismic reflection data. One specific inversion process is the seismic migration, which moves ${ }^{1}$ the reflections in the time section to the correct reflector positions, thus the migration output provides a focused image of the subsurface. A specific type of migration, amplitude-preserving migration, also allows the reconstruction of the reflection amplitudes. Since reflection coefficients for one wave-type (e.g., a $\mathrm{P}$ wave) depend on the $\mathrm{P}$ and $\mathrm{S}$ velocities of the media above and below the reflector, the reconstructed reflection coefficient is a prime source of information: it provides estimates on the $\mathrm{P}$ and $\mathrm{S}$ velocities also below the reflector. Thus, information about shear properties can even be obtained without measuring shear waves, from PP reflections. This feature makes amplitude preserving migration one of the key techniques in seismic imaging.

Amplitude-preserving migration is carried out in terms of a weighted summation of the seismic traces, which are stacked along the diffraction time surface for the image point under consideration. The stacking process yields the structural image whereas the weight functions take care of the amplitudes. Unfortunately this process is very expensive with respect to computational time and storage, because

1. the diffraction time surface for each subsurface point must be computed and stored,

2. individual weight functions must be computed and stored for each source-subsurfacereceiver point combination,

3. the summation stack has to be carried out over the whole experiment's aperture.

\footnotetext{
${ }^{1}$ to migrate means to move
} 
Therefore an efficient strategy is of utmost importance when dealing with migration, especially if amplitudes are concerned.

Different approaches have been published to overcome these problems. A variety of fast traveltime tools have been developed (for an overview, see, e.g., Leidenfrost et al. 1999). Several methods for traveltime interpolation have also been proposed, e.g., parabolic and hyperbolic approximations (Ursin, 1982; Gjøystdal et al., 1984; Schleicher et al., 1993b) or Fourier (sinc) interpolation (Brokešová, 1996) to name only a few. Since the standard method to compute the weight functions is to perform dynamic ray tracing for each point under consideration, much effort has gone into finding simplified, more economic expressions for them, as shown by, e.g., Dellinger et al. (2000) or Zhang et al. (2000). Hanitzsch et al. (2001) introduce an interesting method that applies the weight after the stacking. This solves the problem associated with the computation and storage of large weight function tables, but not with the traveltimes and the aperture. Schleicher et al. (1997) have published a technique to carry out limited aperture migration. They compute the optimum migration aperture together with the weight functions using dynamic ray tracing.

I present an approach to amplitude preserving migration that leads to considerable savings, up to a factor of $10^{-5}$, in storage, as well as in CPU requirements. My strategy uses coarse gridded traveltime tables as only input information. These traveltime tables are in any event needed for the construction of the diffraction time surface. The information that is contained in the traveltimes provides a solution to all of the three major problems stated above:

- a fast and accurate traveltime interpolation algorithm,

- an efficient technique for the determination of the weight functions,

- a technique to determine the optimum migration aperture.

The traveltime interpolation coefficients are determined from the coarse gridded input traveltimes. The coefficients are the input for the computation of the weight functions and the size of the optimum aperture. Since all quantities can be computed on-the-fly no additional storage is required. The following agenda gives a brief overview of the steps involved.

Since the method that I present in this thesis is based on the ray concept the opening chapter, Chapter 2, summarises the basic principles of wave propagation in the high frequency limit, the asymptotic ray method. That chapter is mainly intended to recollect those foundations that are crucial for this thesis, focusing on the ray propagator formalism and the paraxial ray method.

One result from the paraxial ray method is a formula for traveltime interpolation. In the following chapter, Chapter 3, I introduce two methods of traveltime interpolation based on a hyperbolic and a parabolic traveltime expansion, where the latter is closely related to 
the paraxial traveltimes. The interpolation coefficients of the traveltime equation are the fundament on which the remaining chapters of this thesis are based. Therefore emphasis is given to their determination from coarse gridded traveltime tables and to the accuracy of the coefficients that can be achieved. I demonstrate the traveltime interpolation with examples ranging from velocity models with analytic solutions to a 3-D extension of the highly complex Marmousi model. Parts of this chapter were published in Vanelle and Gajewski (2002a).

As stated above, the ray propagator concept is a key element for this thesis. It describes dynamic wavefield properties in terms of second order traveltime derivatives, which are also provided by the traveltime expansion from Chapter 3. In Chapter 4 I make use of the formal similarity between the parabolic traveltime interpolation and the paraxial formula to introduce a relationship between the traveltime coefficients and the ray propagator matrix. As a consequence the complete ray propagator can be determined from coarse gridded traveltimes. Since the quantities required for amplitude preserving migration can be expressed by the ray propagator, the results from this chapter together with the traveltime expressions provide the foundation for the traveltime-based true-amplitude migration.

The traveltime interpolation introduced in Chapter 3 is exact up to second order, thus acknowledging the wavefront curvature. This property translates directly into an expression for the geometrical spreading, which is a consequence of the wavefront curvature. I derive expressions for the spreading in Chapter 5. Examples for simple and complex velocity models illustrate this technique for the computation of geometrical spreading. Some of these results have also been published in Vanelle and Gajewski (1999).

With the results from the previous chapters it is now possible to formulate the technique of traveltime-based true-amplitude migration. Chapter 6 begins with a review of the concept of amplitude-preserving migration. I derive an expression for the weight functions in terms of the traveltime coefficients and give examples for the reconstruction of amplitudes for PP reflections and reflections from PS converted waves. The chapter concludes with remarks concerning the application of the method to complex models. Results from this chapter are also published in Vanelle and Gajewski (2001b) and Vanelle and Gajewski (2002b).

Although the computational effort required for amplitude-preserving migration can be considerably reduced by applying the traveltime-based technique introduced in Chapter 6, a further significant reduction is possible by limiting the migration aperture. Therefore, limited-aperture migration is considered in the following chapter, Chapter 7. I explain why use of a limited aperture is possible and how an optimum aperture can be defined, as well as determined. As for the migration weights, the traveltime coefficients provide a tool to estimate the optimum aperture. Examples illustrate the limited aperture migration and confirm the high potential savings in computational time as well as an enhanced image quality. Parts of this chapter are published in Vanelle and Gajewski (2001a). 
The applications so far were based on traveltime tables as source for the required quantities in terms of traveltime coefficients. Chapter 8 suggests an alternative process to determine the coefficients directly from data. The process is based on the commonreflection-surface (CRS) stack (Jäger et al., 2001; Zhang et al., 2001). The operator used for this stack is a hyperbolic formula describing the reflection move-out. The chapter begins with introducing the hyperbolic traveltime expression from Chapter 3 as a general move-out formula which is then related to other existing NMO equations, and, finally, the CRS operator. Parts of this chapter have been published in Gajewski and Vanelle (2001).

Chapter 9 draws the final conclusions of the work presented in this thesis. It is followed by an outlook in Chapter 10. In this outlook I consider three major aspects of possible future work. One topic that was marginally brushed in the thesis - and to my knowledge nowhere else so far - is the influence of errors in the velocity model on the recovered amplitudes. Another future aspect is a migration implementation that allows for a better illumination of the image point in the subsurface. It was suggested by Brandsberg-Dahl et al. (2001) and is especially suited for a traveltime based implementation. The third point considers the extension of my method to anisotropic media.

A summary of the nomenclature used in this work is given in Appendix A. Appendix $\mathrm{B}$ explains the configuration matrices that describe source and receiver coordinates in terms of the trace coordinates associated with the measurement configuration (e.g., common-shot). In Appendix C I give analytic traveltime coefficients for simple types of media (constant velocity gradient, homogeneous model), where an analytic solution for the traveltimes exists. Finally, Appendix D contains the explicit expressions for the determination of the coefficients from traveltimes.

After the original thesis was written, I have derived an alternative formulation for the weight functions which does not any longer require a priori information on the reflector orientation. Since this new formulation is a major improvement of the technique, I have included it in an additional appendix, Appendix E. 


\section{Chapter 2}

\section{A Review of the Ray Method}

This chapter gives an overview of the concept on that much of this work is based. It introduces the basic equations of asymptotic ray theory and the paraxial ray method. Beginning with the elastodynamic equation, the eikonal equations, and the transport equations for arbitrary elastic anisotropic media will be derived and discussed. This is followed by a section on kinematic and dynamic ray tracing in Cartesian and ray centred coordinates to solve the eikonal and transport equations. The ray propagator formalism and its relation to the paraxial ray approximation is introduced for two different concepts. The first approach is based on a traveltime expansion into the wavefront. In the second, the traveltimes are expanded into reference surfaces, as, e.g., registration surfaces or reflectors.

Since there exists a large variety of texts on the basic concepts of the ray method, I have only given a few representative references at the end of each section. Those are by no means a complete list.

In this thesis I consider amplitude preserving migration in isotropic media. In order to retain the overview character of this chapter, some results in the following sections are derived for isotropic media only, although according formulations for anisotropic media exist. Often, e.g., for the ray propagator formalism, the anisotropic relations have the same form as in the isotropic case but I have not explicitly pointed this out in every case. However, corresponding relationships for anisotropic media can in detail be found in Červený (2001).

\subsection{The Elastodynamic Equation and Ray Series}

Consider a perfectly elastic, inhomogeneous medium that is described by the density $\rho$ and the elasticity tensor $c_{i j k l}$. These are assumed to be continuous functions of space only and to have continuous first and piece-wise continuous second order derivatives. In this case the elastodynamic equation for the displacement $\hat{\boldsymbol{u}}$ (without voluminal forces) reads

$$
\sigma_{i j, j}=\rho u_{i, t t},
$$


where the stress tensor $\sigma_{i j}$ is given by

$$
\sigma_{i j}=c_{i j k l} \varepsilon_{k l}
$$

and the strain tensor $\varepsilon_{k l}$ by

$$
\varepsilon_{k l}=\frac{1}{2}\left(u_{k, l}+u_{l, k}\right)
$$

Both, stress and strain tensors are symmetric tensors. Their symmetry and energy considerations lead to symmetric properties for the elasticity tensor:

$$
c_{i j k l}=c_{j i k l}=c_{i j l k}=c_{k l i j}
$$

As a consequence, the number of independent elastic parameters is reduced from 81 $\left(=3^{4}\right)$ to 21 .

Inserting Equations (2.1.2) and (2.1.3) into (2.1.1) leads to

$$
\left(c_{i j k l} u_{k, l}\right)_{, j}=\rho u_{i, t t}
$$

Equation (2.1.5) is the elastodynamic equation for a perfectly elastic, inhomogeneous, anisotropic medium. One possible way to solve this equation is the ray series solution, an asymptotic series in powers of inverse frequency, which approximates the displacement

$$
u_{i}\left(x_{j}, t\right)=\mathrm{e}^{-i \omega\left(t-\tau\left(x_{j}\right)\right)} \sum_{n=0}^{\infty} \frac{1}{(-i \omega)^{n}} U_{i}^{(n)}\left(x_{j}\right) .
$$

The vectorial amplitude $U_{i}^{(n)}$ is of the order $n$ term (the superscripted $(n)$ does not denote differentiation) and can be complex valued. The quantity $\tau$ is a real scalar called the eikonal or phase function. Surfaces of constant $\tau$ with $\tau(\hat{\boldsymbol{x}})=t_{0}$ represent the wavefront for a specified time $t_{0}$. Since $\tau$ and $U_{i}$ are functions of space only, Equation (2.1.6) separates the spatial dependency from the temporal dependency of the displacement, making (2.1.5) easier to handle. Equation (2.1.6) is a high frequency solution of (2.1.5), where "high" is large in a relative sense. This means, that the variation of the amplitude $u_{i}$, over the distance of a wavelength $\lambda=2 \pi V / \omega$ - more precisely, the maximum wavelength - must be negligible compared to the value of $u_{i}$. This also applies to other related quantities, as, e.g., the phase velocity $V$, and the slowness vector $p_{i}$ which will be explained later. As for the displacement, their variation must also not take place on a smaller scale than that given by the maximum wavelength. This condition reads

$$
\lambda \ll \frac{u_{i}}{\left|\vec{\nabla} u_{i}\right|} \quad, \quad \lambda \ll \frac{p_{i}}{\left|\vec{\nabla} p_{i}\right|} \quad, \quad \text { and } \quad \lambda \ll \frac{V}{|\vec{\nabla} V|} .
$$

Denoting the minimum of the right hand sides in (2.1.7) by the scale length $L$, the three relations (2.1.7) can be summarised to

$$
\frac{\lambda}{L} \ll 1
$$


If in this sense $\omega$ is large enough, only the leading term of the series (2.1.6), i.e. $n=0$, is of practical interest. This is the case for most applications, including those addressed in this work. Therefore from now on only the zero order solution will be considered and the index (0) will be omitted

$$
u_{i}=U_{i}^{(0)} \mathrm{e}^{-i \omega(t-\tau)}=U_{i} \mathrm{e}^{-i \omega(t-\tau)} .
$$

Inserting Equation (2.1.9) into (2.1.5) leads to

$$
(i \omega)^{2} N_{i}(\hat{\boldsymbol{U}})+i \omega M_{i}(\hat{\boldsymbol{U}})+L_{i}(\hat{\boldsymbol{U}})=0
$$

introducing the three vector operators

$$
\begin{aligned}
N_{i}(\hat{\boldsymbol{U}}) & =\frac{c_{i j k l}}{\rho} \tau_{, j} \tau_{, l} U_{k}-U_{i}, \\
M_{i}(\hat{\boldsymbol{U}}) & =\frac{c_{i j k l}}{\rho} \tau_{, j} U_{k, l}+\frac{1}{\rho}\left(c_{i j k l} \tau_{, l} U_{k}\right)_{, j}, \\
L_{i}(\hat{\boldsymbol{U}}) & =\frac{1}{\rho}\left(c_{i j k l} U_{k, l}\right)_{, j} .
\end{aligned}
$$

Since $\omega$ is assumed to be sufficiently large, the $L_{i}(\hat{\boldsymbol{U}})$ term in $(2.1 .10)$ can be neglected. Then $\tau\left(x_{j}\right)$ and $U_{i}\left(x_{j}\right)$ can be determined independent of the frequency by solving

$$
\begin{aligned}
& N_{i}(\hat{\boldsymbol{U}})=0 \\
& M_{i}(\hat{\boldsymbol{U}})=0 .
\end{aligned}
$$

Equation (2.1.14) leads to equations for $\tau$, the eikonal equations, which will be discussed in the following section. The amplitude $U_{i}$ obeys the transport equations that are derived from (2.1.15) and discussed thereafter.

Literature for Section 2.1:

- Červený (1972),

- Pšenčík (1994).

\subsection{The Eikonal Equations}

Equation (2.1.14) can be rewritten to formulate an eigenvalue problem. Introducing the Christoffel matrix $\Gamma_{i k}$ with

$$
\Gamma_{i k}=\frac{c_{i j k l}}{\rho} \tau_{, j} \tau_{, l}
$$

Equation (2.1.14) becomes

$$
\left(\Gamma_{i k}-\delta_{i k}\right) U_{k}=0
$$

Nontrivial solutions of (2.2.2) require that

$$
\left|\Gamma_{i k}-\delta_{i k}\right|=0
$$


Therefore, the $m$ eigenvalues $G^{(m)}$ given by

$$
\left|\Gamma_{i k}-G^{(m)} \delta_{i k}\right|=0
$$

must equal 1. The corresponding eigenvectors $g_{k}^{(m)}$ describe the polarisation. They are determined from

$$
\left(\Gamma_{i k}-G^{(m)} \delta_{i k}\right) g_{k}^{(m)}=0
$$

Expression (2.2.4) yields a cubic equation, meaning that three independent solutions exist for $m=1,2,3$ with eigenvectors that are perpendicular to each other. If two eigenvalues coincide (degeneration, e.g., for the shear wave in an isotropic medium, see below), only the plane that contains the corresponding eigenvectors can be determined. It is perpendicular to the third eigenvector.

Multiplication of Equation (2.2.5) with $g_{k}^{(m)}$ leads to the eikonal equation for anisotropic media

$$
G^{(m)}=1=\Gamma_{i k} g_{i}^{(m)} g_{k}^{(m)}=\frac{c_{i j k l}}{\rho} \tau_{, j} \tau_{, l} g_{i}^{(m)} g_{k}^{(m)}
$$

Let us take a look at the isotropic case now. In isotropic media, the elasticity tensor reduces to

$$
c_{i j k l}=\lambda \delta_{i j} \delta_{k l}+\mu\left(\delta_{i k} \delta_{j l}+\delta_{i l} \delta_{j k}\right)
$$

with the Lamé parameters $\lambda$ and $\mu$ and Kronecker $\delta_{i j}$. A more appealing form is (with $m \neq n)$

$$
\begin{aligned}
c_{n n n n} & =\lambda+2 \mu, \\
c_{m n m n}=c_{m n n m} & =\mu, \\
c_{m m n n} & =\lambda
\end{aligned}
$$

(no summation convention is applied here). The remaining elements of $c_{i j k l}$ are zero. With (2.2.8), Equation (2.1.14) can be rewritten to

$$
\frac{\lambda+\mu}{\rho} \tau_{, i} \tau_{, k} U_{k}+\frac{\mu}{\rho} \tau_{, k} \tau_{, k} U_{i}-U_{i}=0
$$

or, in vectorial form

$$
\frac{\lambda+\mu}{\rho}(\hat{\boldsymbol{U}} \cdot \vec{\nabla} \tau) \vec{\nabla} \tau+\frac{\mu}{\rho}\left(\vec{\nabla}^{2} \tau\right) \hat{\boldsymbol{U}}-\hat{\boldsymbol{U}}=0
$$

To determine the eikonal equations, the scalar product and the vector product of Equation (2.2.10) with $\vec{\nabla} \tau$ is built. The quantity

$$
\hat{\boldsymbol{p}}=\vec{\nabla} \tau
$$

is the slowness vector perpendicular to the wavefront. Its scalar and vector product with $(2.2 .10)$ yields

$$
\begin{aligned}
& \hat{\boldsymbol{N}}(\hat{\boldsymbol{U}}) \cdot \vec{\nabla} \tau=\left[\left(-\rho+(\lambda+2 \mu)(\vec{\nabla} \tau)^{2}\right](\hat{\boldsymbol{U}} \cdot \vec{\nabla} \tau)=0\right. \\
& \hat{\boldsymbol{N}}(\hat{\boldsymbol{U}}) \times \vec{\nabla} \tau=\left[\left(-\rho+\mu(\vec{\nabla} \tau)^{2}\right](\hat{\boldsymbol{U}} \times \vec{\nabla} \tau)=0 .\right.
\end{aligned}
$$

Both Equations (2.2.12) and (2.2.13) must be fulfilled for all $\hat{\boldsymbol{U}}$ and $\tau$. This leads to two independent solutions 
1. $\hat{\boldsymbol{U}} \times \vec{\nabla} \tau=0$ and

$$
(\vec{\nabla} \tau)^{2}=\frac{\lambda+2 \mu}{\rho}=\frac{1}{V_{\mathrm{P}}^{2}}
$$

The first condition demands that $\hat{\boldsymbol{U}}$ is parallel to $\vec{\nabla} \tau$. The polarisation vector $g_{k}^{(3)}=\tau_{, k} / V_{\mathrm{P}}$ is the tangent vector ( $m$ was assigned to be 3 as usual in the literature). This solution describes a $\mathrm{P}$-wave with the propagation velocity $V_{\mathrm{P}}$.

2. $\hat{\boldsymbol{U}} \cdot \vec{\nabla} \tau=0$ and

$$
(\vec{\nabla} \tau)^{2}=\frac{\mu}{\rho}=\frac{1}{V_{\mathrm{S}}^{2}}
$$

Here, the first condition demands that $\hat{\boldsymbol{U}}$ is perpendicular to $\vec{\nabla} \tau$, corresponding to a shear wave that has two components with the polarisation vectors $g_{k}^{(1)}$ and $g_{k}^{(2)}$ and the propagation velocity $V_{\mathrm{S}}$. As mentioned before, in this case the polarisation vectors can be chosen arbitrarily, perpendicular to each other, in the plane perpendicular to $\vec{\nabla} \tau$. One particular choice are the base vectors $\overrightarrow{\boldsymbol{e}}_{1}$ and $\overrightarrow{\boldsymbol{e}}_{2}$ from the ray centred coordinate system (see below) which has advantages concerning the transport equations that will be derived in the next section.

Equations (2.2.14) and (2.2.15) are the isotropic eikonal equations for $\mathrm{P}$ and $\mathrm{S}$ waves, respectively. Equation (2.2.6) for isotropic media reads

$$
G^{(m)}=1=V_{m}^{2} \tau_{, k} \tau_{, k}
$$

Here, the velocity $V_{m}$ is the phase velocity of the corresponding wave, i.e., $V_{3}=V_{\mathrm{P}}$ and $V_{1}=V_{2}=V_{\mathrm{S}}$.

Literature for Section 2.2:

- Červený and Ravindra (1971),

- Gajewski and Pšenčík (1987).

\subsection{The Transport Equations}

The procedure for the determination of the transport equations in inhomogeneous anisotropic media is similar to the derivation of the eikonal equations in the previous section. The displacement vectors $U_{i}^{(m)}\left(x_{j}\right)$ can be written as

$$
U_{i}^{(m)}\left(x_{j}\right)=U^{(m)}\left(x_{j}\right) g_{i}^{(m)}\left(x_{j}\right)
$$

Since this applies to all three waves, $m=1,2,3$, the index $m$ will be omitted from now on. Building the scalar product of the polarisation vector $g_{i}$ and Equation (2.1.15) leads to

$$
M_{i}(\hat{\boldsymbol{U}}) g_{i}=2 U_{, j} v_{j}+\frac{U}{\rho} v_{j} \rho_{, j}+U v_{j, j}=0
$$


introducing the group velocity vector

$$
v_{i}=\frac{\mathrm{d} x_{i}}{\mathrm{~d} \tau}=\frac{c_{i j k l}}{\rho} \tau_{, l} g_{j} g_{k}
$$

In isotropic media, the group velocity $v=|\hat{\boldsymbol{v}}|$ equals the phase velocity $V$. Note that for shear waves $(m=1,2)$ the choice of polarisation vectors is important. For arbitrary choice, there are additional terms in (2.3.2), leading to two coupled transport equations for $m=1$ and $m=2$. Only if $\mathrm{d} g_{i}^{(1,2)} / \mathrm{d} s \propto p_{i}$ is fulfilled (as it is the case for the ray centred coordinates, see Section 2.5 below), Equations (2.3.2) decouple. Equations (2.3.2) for $m=1,2,3$ are the transport equations. They can be rewritten in terms of $\tau$ derivatives which is easier to be solved. To the first two terms in (2.3.2)

$$
\Phi_{, j} v_{j}=\vec{\nabla} \Phi \cdot \frac{\mathrm{d} \hat{\boldsymbol{x}}}{\mathrm{d} \tau}=\frac{\mathrm{d} \Phi}{\mathrm{d} \tau}
$$

applies. The divergence of the group velocity in the third term of (2.3.2) is given by

$$
v_{j, j}=\lim _{\Delta V_{\tau} \rightarrow 0} \frac{1}{\Delta V_{\tau}} \oint \hat{\boldsymbol{v}} \cdot \mathrm{d} \hat{\boldsymbol{S}}_{\tau}=\lim _{\Delta V_{\tau} \rightarrow 0} \frac{1}{\Delta V_{\tau}} \oint \hat{\boldsymbol{v}} \cdot \hat{\boldsymbol{p}} V \mathrm{~d} S_{\tau}
$$

where the integration is carried out over the surface of a piece of a ray tube that is limited by the phase fronts $t=\tau_{0}$ and $t=\tau_{0}+\mathrm{d} \tau=\tau$ (see Figure 2.1). A vectorial surface element $\mathrm{d} \boldsymbol{S}_{\gamma_{3}}$ is the cross-sectional area of the ray tube: the part of the surface $\gamma_{3}=$ const. that is cut out by the ray tube. It is defined as

$$
\mathrm{d} \hat{\boldsymbol{S}}_{\gamma_{3}}=\left(\frac{\partial \hat{\boldsymbol{x}}}{\partial \gamma_{1}} \times \frac{\partial \hat{\boldsymbol{x}}}{\partial \gamma_{2}}\right)_{\gamma_{3}} \mathrm{~d} \gamma_{1} \mathrm{~d} \gamma_{2}
$$

introducing the ray coordinates (ray parameters) $\gamma_{i}$, see also Figure 2.1. The corresponding volume element is given by

$$
\mathrm{d} V_{\gamma_{3}}=\mathcal{J}_{\gamma_{3}} \mathrm{~d} \gamma_{1} \mathrm{~d} \gamma_{2} \mathrm{~d} \gamma_{3}=\mathrm{d} \hat{\boldsymbol{S}}_{\gamma_{3}} \cdot \frac{\mathrm{d} \hat{\boldsymbol{x}}}{\mathrm{d} \gamma_{3}} \mathrm{~d} \gamma_{3}
$$

where $\mathcal{J}_{\gamma_{3}}$ is the determinant of the Jacobian $\underline{\hat{X}}$

$$
X_{i j}=\frac{\partial x_{i}}{\partial \gamma_{j}}
$$

Choice of the arc length $s$ for $\gamma_{3}$ leads to

$$
\mathcal{J}_{s} \mathrm{~d} \gamma_{1} \mathrm{~d} \gamma_{2}=\mathrm{d} \hat{\boldsymbol{S}}_{s} \cdot \frac{\mathrm{d} \hat{\boldsymbol{x}}}{\mathrm{d} s}=\mathrm{d} \hat{\boldsymbol{S}}_{s} \cdot \frac{\hat{\boldsymbol{v}}}{v}=\mathrm{d} S_{s}
$$

and for $\gamma_{3}=\tau$

$$
\begin{aligned}
\mathcal{J}_{\tau} \mathrm{d} \gamma_{1} \mathrm{~d} \gamma_{2} & =\mathrm{d} \hat{\boldsymbol{S}}_{\tau} \cdot \frac{\mathrm{d} \hat{\boldsymbol{x}}}{\mathrm{d} s}=v \mathrm{~d} \hat{\boldsymbol{S}}_{\tau} \cdot \frac{\mathrm{d} \hat{\boldsymbol{x}}}{\mathrm{d} s}=v \mathrm{~d} S_{s} \\
& =\mathrm{d} S_{\tau} V \hat{\boldsymbol{p}} \cdot \hat{\boldsymbol{v}}=V \mathrm{~d} S_{\tau}=\Omega V \mathrm{~d} \gamma_{1} \mathrm{~d} \gamma_{2}
\end{aligned}
$$




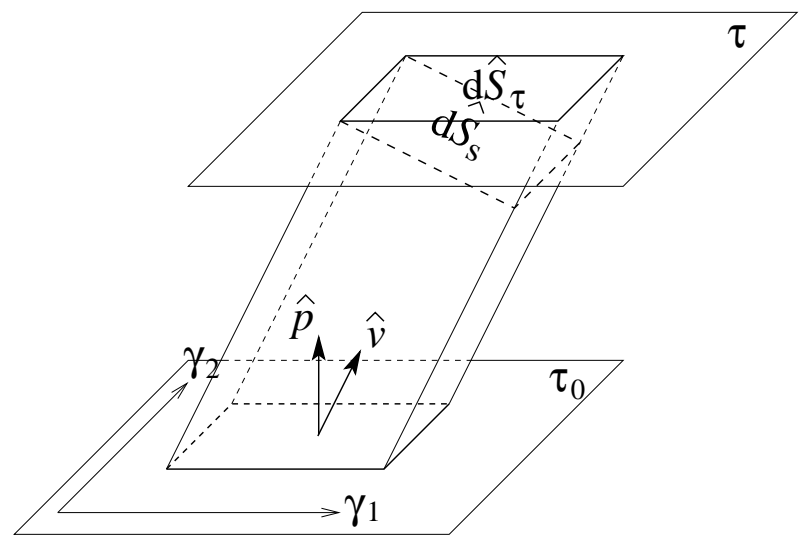

Figure 2.1: A segment of a ray tube limited by cross sections with the phase front at $t=\tau=\tau_{0}+\mathrm{d} \tau$ and $t=\tau_{0}$. The corners of the surface element $\mathrm{d} \hat{\boldsymbol{S}}_{\gamma_{3}}$ are given by rays with the ray parameters $\left(\gamma_{1}, \gamma_{2}\right),\left(\gamma_{1}+\mathrm{d} \gamma_{1}, \gamma_{2}\right),\left(\gamma_{1}+\gamma_{2}+\mathrm{d} \gamma_{2}\right)$, and $\left(\gamma_{1}+\mathrm{d} \gamma_{1}, \gamma_{2}+\mathrm{d} \gamma_{2}\right)$.

introducing the quantity $\Omega$

$$
\Omega=\left(\frac{\partial \hat{\boldsymbol{x}}}{\partial \gamma_{1}} \times \frac{\partial \hat{\boldsymbol{x}}}{\partial \gamma_{2}}\right)_{\tau} \cdot \hat{\boldsymbol{p}} V=\frac{\mathrm{d} S_{\tau}}{\mathrm{d} \gamma_{1} \mathrm{~d} \gamma_{2}}
$$

Now the volume element $\Delta V_{\tau}$ can be written as

$$
\Delta V_{\tau}=V \mathrm{~d} S_{\tau} \Delta \tau=V \mathrm{~d} S_{\tau}\left(\tau-\tau_{0}\right)
$$

Figure 2.1 shows that $\hat{\boldsymbol{v}} \mathrm{d} \hat{\boldsymbol{S}}_{\tau}$ has only non-vanishing contributions at the cross sections of the ray tube with the phase fronts $t=$ const.

$$
\text { at } \quad \tau: \quad \hat{\boldsymbol{v}} \cdot \mathrm{d} \hat{\boldsymbol{S}}_{\tau}=V(\tau) \mathrm{d} S_{\tau}=\mathcal{J}_{\tau}(\tau) \mathrm{d} \gamma_{1} \mathrm{~d} \gamma_{2},
$$

and

$$
\text { at } \quad \tau_{0}: \quad \hat{\boldsymbol{v}} \cdot \mathrm{d} \hat{\boldsymbol{S}}_{\tau}=-V\left(\tau_{0}\right) \mathrm{d} S_{\tau}=-\mathcal{J}_{\tau}\left(\tau_{0}\right) \mathrm{d} \gamma_{1} \mathrm{~d} \gamma_{2}
$$

Inserting these and (2.3.13) into (2.3.5) yields

$$
\begin{aligned}
v_{j, j} & =\lim _{\tau \rightarrow \tau_{0}} \frac{\mathcal{J}_{\tau}(\tau)-\mathcal{J}_{\tau}\left(\tau_{0}\right)}{\mathcal{J}_{\tau} \cdot\left(\tau-\tau_{0}\right)} \\
& =\frac{1}{\mathcal{J}_{\tau}} \frac{\mathrm{d} \mathcal{J}_{\tau}}{\mathrm{d} \tau} .
\end{aligned}
$$

With (2.3.16) and (2.3.4), Equation (2.3.2) becomes

$$
2 \frac{\mathrm{d} U}{\mathrm{~d} \tau}+\frac{U}{\rho} \frac{\mathrm{d} \rho}{\mathrm{d} \tau}+\frac{U}{\mathcal{J}_{\tau}} \frac{\mathrm{d} \mathcal{J}_{\tau}}{\mathrm{d} \tau}=0
$$

or

$$
\frac{\mathrm{d}}{\mathrm{d} \tau}\left(\sqrt{\rho \mathcal{J}_{\tau}} U\right)=0
$$


The solution of Equation (2.3.18) is straightforward

$$
U(\tau)=U\left(\tau_{0}\right) \sqrt{\frac{\rho\left(\tau_{0}\right) \mathcal{J}_{\tau}\left(\tau_{0}\right)}{\rho(\tau) \mathcal{J}_{\tau}(\tau)}} .
$$

Equation (2.3.19), however may lead to problems if, e.g., a point source at $\tau_{0}$ is considered. For this case it is better to use the following solution of (2.3.18)

$$
U(\tau)=\frac{\Psi\left(\gamma_{1}, \gamma_{2}\right)}{\sqrt{\rho(\tau) \mathcal{J}_{\tau}(\tau)}}=\frac{\Psi\left(\gamma_{1}, \gamma_{2}\right)}{\sqrt{\rho(\tau) V(\tau) \Omega(\tau)}}
$$

where the function $\Psi\left(\gamma_{1}, \gamma_{2}\right)$ is constant along the ray specified by the ray parameters $\left(\gamma_{1}, \gamma_{2}\right)$, e.g., the take-off angles. If the arc length $s$ is chosen as $\gamma_{3}$ instead of $\tau$, Equation (2.3.20) becomes

$$
U(\tau)=\frac{\Psi\left(\gamma_{1}, \gamma_{2}\right)}{\sqrt{\rho(\tau) v(\tau) \mathcal{J}_{s}(\tau)}},
$$

since $\mathcal{J}_{\tau}=v \mathcal{J}_{s}$ follows from (2.3.10) and (2.3.9). At this point the relative geometrical spreading $L$ is introduced as

$$
L=\sqrt{\Omega} .
$$

Since the sign of $\mathcal{J}_{\gamma_{3}}$ may change, it is suitable to take the square root of $\mathcal{J}_{\gamma_{3}}$ as follows

$$
\sqrt{\mathcal{J}_{\gamma_{3}}}=\sqrt{\left|\mathcal{J}_{\gamma_{3}}\right|} \mathrm{e}^{-i \frac{\pi}{2} \kappa}
$$

where the quantity $\kappa$, the so-called KMAH-index is an index of the ray trajectory. It gives the number of points at which the sign of $\mathcal{J}_{\gamma_{3}}$ has changed. These points are called caustic points. A first order caustic increments $\kappa$ by one, a second order caustic by two. The behaviour of a ray tube at caustic points of first and second order is illustrated in Figure 2.2 .

Literature for Section 2.3:

- Červený (1972),

- Pšenčík (1994).

\section{$2.4 \quad$ Ray Tracing}

The phase function $\tau\left(x_{i}\right)$ can be found by solving the kinematic ray tracing (KRT) equations. They are easily obtained from applying the method of characteristics (Courant and Hilbert, 1962) to the eikonal Equation (2.2.6). If a Hamiltonian $\mathcal{H}\left(x_{i}, p_{i}, \tau\right)=0$ can be found, the characteristics in terms of the parameter $u$ are described by

$$
\frac{\mathrm{d} x_{i}}{\mathrm{~d} u}=\frac{\partial \mathcal{H}}{\partial p_{i}} \quad, \quad \frac{\mathrm{d} p_{i}}{\mathrm{~d} u}=-\frac{\partial \mathcal{H}}{\partial x_{i}} \quad, \quad \frac{\mathrm{d} \tau}{\mathrm{d} u}=p_{i} \frac{\partial \mathcal{H}}{\partial p_{i}} .
$$

One possible choice of a Hamiltonian is

$$
\mathcal{H}\left(x_{i}, p_{i}\right)=G-1=a_{i j k l} p_{j} p_{l} g_{i} g_{k}=0,
$$



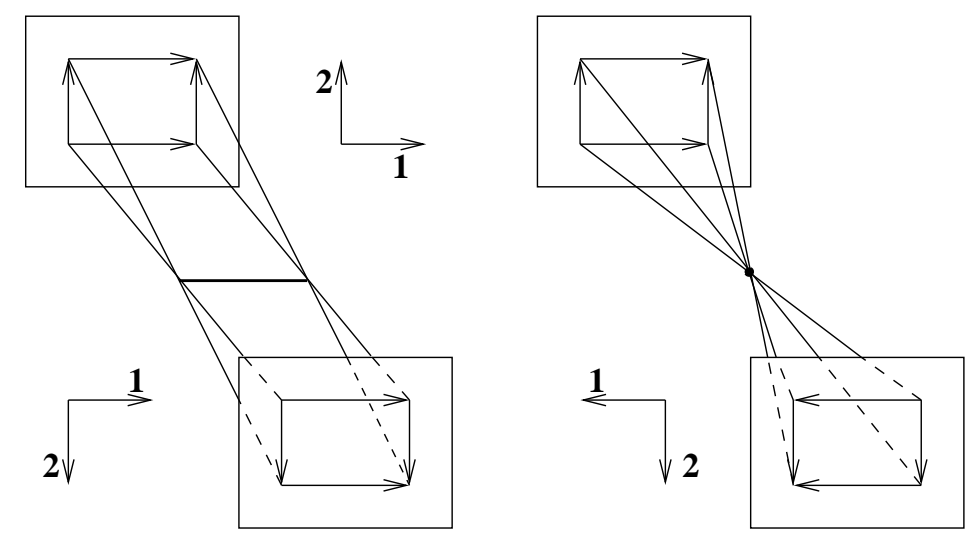

Figure 2.2: Behaviour of a ray tube at caustic points: at a first order caustic (line caustic) the ray tube shrinks to an arc perpendicular to the direction of propagation (left). At a second order caustic (point caustic) the ray tube shrinks to a point (right). The vectors indicate that after going through a line caustic the direction of the surface element is changed, leading to a change in sign of $\mathcal{J}_{\gamma_{3}}$. If the ray passes a point caustic, the sign changes twice (resulting in the original sign).

using an eigenvalue of the eikonal equation, $G$, and $a_{i j k l}=c_{i j k l} / \rho$, leading to the following KRT for the parameter $\tau$

$$
v_{i}=\frac{\mathrm{d} x_{i}}{\mathrm{~d} \tau}=a_{i j k l} p_{l} g_{j} g_{k} \quad, \quad \frac{\mathrm{d} p_{i}}{\mathrm{~d} \tau}=-\frac{1}{2} a_{n j k l, i} p_{n} p_{l} g_{j} g_{k}
$$

where $v_{i}$ is the $i$-th component of the group velocity vector. With $\mathrm{d} s / \mathrm{d} \tau=v$, the KRT system for the parameter $s$ reads

$$
\frac{\mathrm{d} x_{i}}{\mathrm{~d} s}=\frac{1}{v} a_{i j k l} p_{l} g_{j} g_{k} \quad, \quad \frac{\mathrm{d} p_{i}}{\mathrm{~d} s}=-\frac{1}{2 v} a_{n j k l, i} p_{n} p_{l} g_{j} g_{k} \quad .
$$

For isotropic media the systems (2.4.3) and (2.4.4) reduce to

$$
\begin{array}{lll}
\frac{\mathrm{d} x_{i}}{\mathrm{~d} \tau}=V^{2} p_{i} & , & \frac{\mathrm{d} p_{i}}{\mathrm{~d} \tau}=-\frac{1}{V} \frac{\partial V}{\partial x_{i}} \\
\frac{\mathrm{d} x_{i}}{\mathrm{~d} s}=V p_{i} & , & \frac{\mathrm{d} p_{i}}{\mathrm{~d} s}=-\frac{1}{V^{2}} \frac{\partial V}{\partial x_{i}}
\end{array} .
$$

To compute the ray amplitude, the quantity $\mathcal{J}_{\gamma_{3}}=\operatorname{det} \underline{\hat{X}}$ needs to be determined. Defining the matrix $\underline{\hat{Y}}$

$$
Y_{i j}=\frac{\partial p_{i}}{\partial \gamma_{j}}
$$

the dynamic ray tracing (DRT) equations are obtained from differentiating the KRT Equations (2.4.3) or (2.4.4) with respect to the ray parameters $\gamma_{J}(J=1,2)$. For, e.g., $\gamma_{3}=\tau$ this yields

$$
\begin{aligned}
\frac{\mathrm{d} X_{i J}}{\mathrm{~d} \tau} & =\frac{1}{2}\left[\frac{\partial^{2} G}{\partial p_{i} \partial x_{k}} X_{k J}+\frac{\partial^{2} G}{\partial p_{i} \partial p_{k}} Y_{k J}\right], \\
\frac{\mathrm{d} Y_{i J}}{\mathrm{~d} \tau} & =-\frac{1}{2}\left[\frac{\partial^{2} G}{\partial x_{i} \partial x_{k}} X_{k J}+\frac{\partial^{2} G}{\partial x_{i} \partial p_{k}} Y_{k J}\right] .
\end{aligned}
$$


The elements $X_{i 3}=\frac{\partial x_{i}}{\partial \tau}$ and $Y_{i 3}=\frac{\partial p_{i}}{\partial \tau}$ can be determined directly from the ray tracing Equations (2.4.3).

For isotropic media the DRT system looks as follows

$$
\begin{aligned}
\frac{\mathrm{d} X_{i J}}{\mathrm{~d} \tau} & =2 V \frac{\partial V}{\partial x_{k}} p_{i} X_{k J}+V^{2} Y_{i J} \\
\frac{\mathrm{d} Y_{i J}}{\mathrm{~d} \tau} & =\left[\frac{1}{V^{2}} \frac{\partial V}{\partial x_{i}} \frac{\partial V}{\partial x_{k}}-\frac{1}{V} \frac{\partial^{2} V}{\partial x_{i} \partial x_{k}}\right] X_{k J} .
\end{aligned}
$$

This equation and (2.4.5) require that the velocity function $V$ must have continuous derivatives up to second order. The behaviour of rays at interfaces where this is not the case is discussed further below.

Literature for Section 2.4:

- Červený (1972),

- Gajewski and Pšenčík (1990).

\subsection{Ray Centred Coordinates}

Sometimes it is convenient to work with a ray centred coordinate system instead of global Cartesian coordinates. This is for example the case if so-called paraxial rays, rays in the near vicinity of a central ray, are to be computed. The radius vector in the ray centred system is given by

$$
\hat{\boldsymbol{r}}\left(q_{1}, q_{2}, s\right)=\hat{\boldsymbol{r}}(0,0, s)+\overrightarrow{\boldsymbol{e}}_{1}(s) q_{1}+\overrightarrow{\boldsymbol{e}}_{2}(s) q_{2} \quad
$$

On the central ray $q_{1}=q_{2}=0$. Whether a ray is in a "near vicinity" of the central ray depends on the model under consideration. The vectors

$$
\begin{aligned}
& \overrightarrow{\boldsymbol{e}}_{1}=\overrightarrow{\boldsymbol{n}} \cos \theta-\overrightarrow{\boldsymbol{b}} \sin \theta \\
& \overrightarrow{\boldsymbol{e}}_{2}=\overrightarrow{\boldsymbol{n}} \sin \theta+\overrightarrow{\boldsymbol{b}} \cos \theta
\end{aligned}
$$

and the tangent vector $\overrightarrow{\boldsymbol{t}}$ form an orthogonal base. Vector $\overrightarrow{\boldsymbol{t}}$ is perpendicular to the wavefront, $\overrightarrow{\boldsymbol{e}}_{I}(I=1,2)$ lie in the wavefront. The vectors $\overrightarrow{\boldsymbol{n}}$ and $\overrightarrow{\boldsymbol{b}}$ are the normal and binormal vector (see Figure 2.3). They and $\overrightarrow{\boldsymbol{t}}$ obey Frenet's formulae. The angle $\theta$ between $\overrightarrow{\boldsymbol{e}}_{1}$ and $\overrightarrow{\boldsymbol{b}}$, as between $\overrightarrow{\boldsymbol{e}}_{2}$ and $\overrightarrow{\boldsymbol{n}}$ can be obtained from the integration over the torsion $T$ along the ray

$$
\theta(s)=\theta\left(s_{0}\right)+\int_{s_{0}}^{s} T(\sigma) \mathrm{d} \sigma
$$


The scale parameters $h_{1}$ and $h_{2}$ (with $\left.h_{I}=\left|\mathrm{d} \hat{\boldsymbol{r}} / \mathrm{d} q_{I}\right|\right)$ are equal to one, and $h_{3}=h$, with the curvature of the ray $K$, is

$$
h_{3}=h=\left|\frac{\mathrm{d} \hat{\boldsymbol{r}}}{\mathrm{d} s}\right|=1-K \cos \theta q_{1}-K \sin \theta q_{2} \quad .
$$

Derivation of the base vectors yields

$$
\begin{array}{ll}
\frac{\mathrm{d} \overrightarrow{\boldsymbol{e}}_{1}}{\mathrm{~d} s}= & -K \cos \theta \overrightarrow{\boldsymbol{t}}=\left(\frac{\mathrm{d} \overrightarrow{\boldsymbol{t}}}{\mathrm{d} s} \cdot \overrightarrow{\boldsymbol{e}}_{1}\right) \overrightarrow{\boldsymbol{t}} \\
\frac{\mathrm{d} \overrightarrow{\boldsymbol{e}}_{2}}{\mathrm{~d} s}=-K \sin \theta \overrightarrow{\boldsymbol{t}}=\left(\frac{\mathrm{d} \overrightarrow{\boldsymbol{t}}}{\mathrm{d} s} \cdot \overrightarrow{\boldsymbol{e}}_{2}\right) \overrightarrow{\boldsymbol{t}} \\
\frac{\mathrm{d} \overrightarrow{\boldsymbol{t}}}{\mathrm{d} s}=K \cos \theta \overrightarrow{\boldsymbol{e}}_{1}+K \sin \theta \overrightarrow{\boldsymbol{e}}_{2}=\left(\frac{\mathrm{d} \overrightarrow{\boldsymbol{t}}}{\mathrm{d} s} \cdot \overrightarrow{\boldsymbol{e}}_{I}\right) \overrightarrow{\boldsymbol{e}}_{I}
\end{array}
$$

The derivatives of $\overrightarrow{\boldsymbol{e}}_{I}$ point in the direction of $\overrightarrow{\boldsymbol{t}}$. This means that - unlike the vectors $\overrightarrow{\boldsymbol{n}}$ and $\overrightarrow{\boldsymbol{b}}$ - the vectors $\overrightarrow{\boldsymbol{e}}_{I}$ do not rotate during propagation along the central ray. Comparison of (2.5.5) to (2.5.4) leads to an expression for $h$

$$
h=1-\left(\frac{\mathrm{d} \overrightarrow{\boldsymbol{t}}}{\mathrm{d} s} \cdot \overrightarrow{\boldsymbol{e}}_{I}\right) q_{I} .
$$

The scale parameter $h$ can be expressed in terms of derivatives of the phase velocity. Because the ray tracing Equations for isotropic media, (2.4.6), are employed in the following step, the resulting relation holds for isotropic media only. Using $\overrightarrow{\boldsymbol{t}}=V \hat{\boldsymbol{p}}$ and the ray tracing Equations (2.4.6), the derivative of the tangent vector in isotropic media becomes

$$
\frac{\mathrm{d} \hat{\boldsymbol{t}}}{\mathrm{d} s}=-\left.\frac{1}{V} \frac{\partial V}{\partial q_{I}}\right|_{q_{K}=0} \overrightarrow{\boldsymbol{e}}_{I}
$$
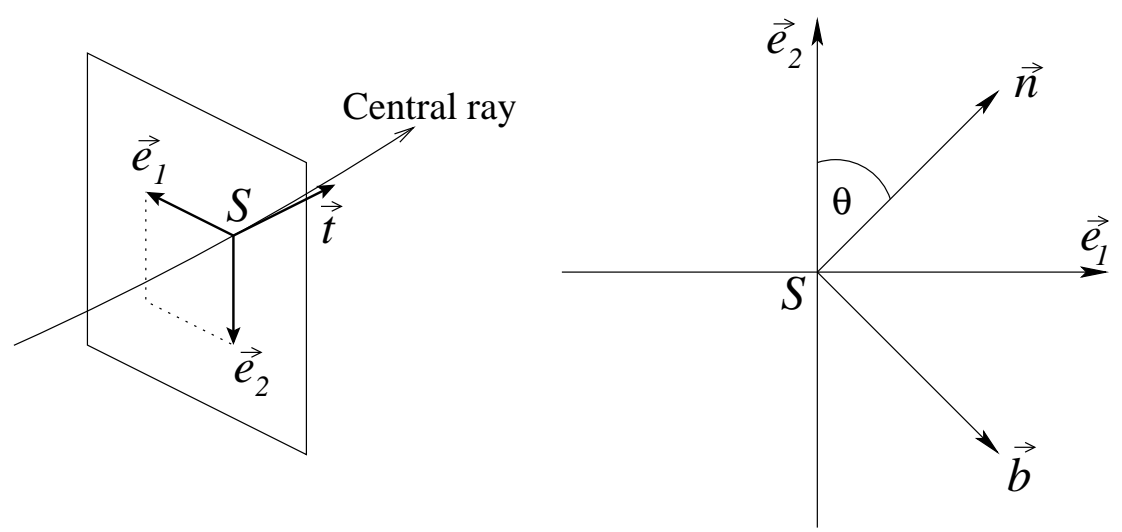

Figure 2.3: Definition of the ray centred coordinate system: position of the base vectors with respect to the central ray and the wavefront. The surface indicates the tangent plane to the wavefront, perpendicular to the central ray (left). Right: unit vectors $\overrightarrow{\boldsymbol{e}}_{1}$ and $\overrightarrow{\boldsymbol{e}}_{2}$, and normal and binormal vectors, $\overrightarrow{\boldsymbol{n}}$ and $\overrightarrow{\boldsymbol{b}}$, respectively. 
and therefore

$$
h=1+\left.\frac{1}{V} \frac{\partial V}{\partial q_{I}}\right|_{q_{K}=0} q_{I} .
$$

On the central ray, the coordinates $q_{I}$ are zero and therefore $h=1$.

In order to derive the ray tracing equations in ray centred coordinates, the Hamilton equations (2.4.1) can be used. I will derive the ray tracing equations for isotropic media only, however, use of a Hamiltonian for anisotropic media will lead to an equivalent formulation for anisotropic media, see, e.g., Červený (2001). A suitable Hamiltonian for isotropic media in the ray centred coordinate system is

$$
\mathcal{H}\left(q_{I}, p_{I}^{(q)}\right)=-\frac{h}{V}\left[1-V^{2} p_{I}^{(q)} p_{I}^{(q)}\right]^{1 / 2}
$$

where $p_{I}^{(q)}$ is the $I$-th slowness component in ray centred coordinates. To derive the ray tracing system for paraxial rays, this Hamiltonian is expanded in $p_{I}^{(q)}$ and $q_{I}$ neglecting terms of higher than second order

$$
\mathcal{H}\left(q_{I}, p_{I}^{(q)}\right)=-\frac{1}{V}+\frac{1}{2} V p_{I}^{(q)} p_{I}^{(q)}+\frac{1}{2 V^{2}} \frac{\partial^{2} V}{\partial q_{I} \partial q_{J}} q_{I} q_{J}
$$

leading to the kinematic ray tracing equations

$$
\begin{aligned}
\frac{\mathrm{d} q_{I}}{\mathrm{~d} s} & =\frac{\partial \mathcal{H}}{\partial p_{I}^{(q)}}=V p_{I}^{(q)} \\
\frac{\mathrm{d} p_{I}^{(q)}}{\mathrm{d} s} & =-\frac{\partial \mathcal{H}}{\partial q_{I}}=-\frac{1}{V} \frac{\partial^{2} V}{\partial q_{I} \partial q_{J}} q_{J} .
\end{aligned}
$$

For the determination of the ray amplitude, the matrix $\underline{\hat{X}}$ is expressed as a product of two matrices $\underline{\hat{H}}$ and $\underline{\hat{Q}}$. The Jacobian of the transformation from Cartesian to ray centred coordinates along the central ray $\left(q_{I}=0\right)$ is denoted by $\underline{\hat{H}}$. Then, the elements of $\underline{\hat{H}}$ are given by

$$
H_{i j}=\frac{\partial x_{i}}{\partial q_{j}}=\frac{\partial q_{j}}{\partial x_{i}}
$$

where $q_{3}=s$. On the central ray, $h=1$, and therefore $\operatorname{det} \underline{\hat{H}}=1$. The matrix $\underline{\hat{Q}}$ is the Jacobian for the transformation from ray centred coordinates to ray coordinates on the central ray with

$$
Q_{i j}=\frac{\partial q_{i}}{\partial \gamma_{j}}
$$

Since $q_{I}=0$ along the central ray, the components $Q_{13}=Q_{23}=0$. Specifying $\gamma_{3}=s$ yields $Q_{33}=1$ and thus

$$
\operatorname{det} \underline{\hat{X}}=\operatorname{det} \underline{\hat{H}} \cdot \operatorname{det} \underline{\hat{Q}}=Q_{11} Q_{22}-Q_{12} Q_{21}=\operatorname{det} \underline{Q} .
$$

This means that the ray amplitude is determined by $\underline{Q}$, which is the upper left $2 \times 2$ submatrix of $\underline{\hat{Q}}$. For the geometrical spreading this means that

$$
L=\sqrt{\operatorname{det} \underline{Q}} .
$$


Note that Equation (2.5.15) is also valid in anisotropic media.

Introducing also the $2 \times 2$ matrix $\underline{P}$ with

$$
P_{I J}=\frac{\partial p_{I}^{(q)}}{\partial \gamma_{J}}
$$

and differentiation of $Q_{I J}$ and $P_{I J}$ with respect to $s$ leads to the DRT system in ray centred coordinates

$$
\frac{\mathrm{d} Q_{I J}}{\mathrm{~d} s}=V P_{I J} \quad, \quad \frac{\mathrm{d} P_{I J}}{\mathrm{~d} s}=-\frac{1}{V^{2}} V_{J K} P_{K J} .
$$

The $2 \times 2$ matrix $\underline{\mathbb{V}}$ contains second order derivatives of the velocity

$$
V_{I J}=\left.\frac{\partial^{2} V}{\partial q_{I} \partial q_{J}}\right|_{q_{K}=0}
$$

The DRT system $(2.5 .17)$ can be rewritten to another form, if the matrix $\underline{M}=\underline{P} \underline{Q}^{-1}$ is introduced. Differentiation of $\underline{M}$ leads to

$$
\frac{\mathrm{d}}{\mathrm{d} s} \underline{\mathrm{M}}+V \underline{\mathrm{M}} \underline{\mathrm{M}}+\frac{1}{V^{2}} \underline{\mathrm{V}}=\underline{0}
$$

which is known as the Ricatti equation. Equation (2.5.19) can also be derived from the paraxial eikonal equation. The eikonal equation in ray centred coordinates reads

$$
\tau_{, 1}^{2}+\tau_{, 2}^{2}+\frac{1}{h^{2}} \tau_{, s}^{2}=\frac{1}{V^{2}}
$$

Taking into consideration that $\tau_{, I}=p_{I}^{(q)},(2.5 .20)$ can be solved for $\tau_{, s}$

$$
\tau_{, s}=\frac{h}{V} \sqrt{1-V^{2} p_{I}^{(q)} p_{I}^{(q)}}=-H\left(q_{I}, p_{I}^{(q)}\right)
$$

where $H\left(q_{I}, p_{I}^{(q)}\right)$ is the Hamiltonian from Equation (2.5.9). Using the approximation of the Hamiltonian (2.5.10) and inserting $\tau_{, I}$ and $\tau_{, s}$ into (2.5.20), this leads to the paraxial eikonal equation

$$
\left(\frac{\partial \tau}{\partial q_{1}}\right)^{2}+\left(\frac{\partial \tau}{\partial q_{2}}\right)^{2}+\left(\frac{\partial \tau}{\partial s}\right)^{2}=\frac{1}{V^{2}}-\frac{1}{V^{3}} V_{I J} q_{I} q_{J}
$$

where the matrix $\underline{\mathrm{V}}$ was defined in (2.5.18). For the following step an expansion of the eikonal $\tau$ is required. Expansion of $\tau$ in $q_{I}$ yields

$$
\tau\left(q_{1}, q_{2}, s\right)=\tau(0,0, s)+\frac{1}{2} M_{I J} q_{I} q_{J}
$$

where the matrix $\underline{\mathrm{M}}(s)$ is the second order derivative matrix of $\tau$ with respect to $q_{I}$

$$
M_{I J}=\left.\frac{\partial^{2} \tau}{\partial q_{I} \partial q_{J}}\right|_{q_{K}=0}
$$


From now on, $\tau(s)$ is used to abbreviate $\tau(0,0, s)$. The first order derivatives disappear because the expansion is carried out in the wavefront, where $\tau$ is constant. Building the derivative of (2.5.23) with respect to $s$ yields

$$
\frac{\partial \tau\left(q_{1}, q_{2}, s\right)}{\partial s}=\frac{\partial \tau(s)}{\partial s}+\frac{1}{2} \frac{\mathrm{d} M_{I J}}{\mathrm{~d} s} q_{I} q_{J}=\frac{1}{V}+\frac{1}{2} \frac{\mathrm{d} M_{I J}}{\mathrm{~d} s} q_{I} q_{J} .
$$

Similarly, derivation of $(2.5 .23)$ with respect to $q_{I}$ yields

$$
\left(\frac{\partial \tau}{\partial q_{1}}\right)^{2}+\left(\frac{\partial \tau}{\partial q_{2}}\right)^{2}=M_{I K} M_{K J} q_{I} q_{J}
$$

Inserting the found expressions for $\tau_{, s}$ and $\tau_{, I}$ into (2.5.22) gives again the Ricatti equation. Therefore, the matrix $\underline{M}$ from (2.5.19) equals the second order derivative matrix introduced in (2.5.23) with

$$
M_{I J}=P_{I K} Q_{K J}^{-1}=\left.\frac{\partial^{2} \tau}{\partial q_{I} \partial q_{J}}\right|_{q_{K}=0} .
$$

Literature for Section 2.5:

- Popov and Pšenčík (1978),

- Červený and Hron (1980).

\subsection{Propagator Matrices}

Introducing the $4 \times 1$ column matrix $\underline{\mathrm{W}}$

$$
\underline{\mathbf{W}}=\left(q_{1}, q_{2}, p_{1}^{(q)}, p_{2}^{(q)}\right)^{\top},
$$

the ray tracing system (2.5.11) can be written as

$$
\frac{\mathrm{d}}{\mathrm{d} s} \underline{\mathrm{W}}=\underline{\underline{\mathrm{S}}} \underline{\mathrm{W}}
$$

where the $4 \times 4$ matrix $\underline{\underline{\mathrm{S}}}$ is given by

$$
\underline{\underline{S}}=\left(\begin{array}{cc}
\underline{0} & \underline{1} \\
-\frac{1}{V^{2}} \underline{V} & \underline{0}
\end{array}\right) .
$$

The matrices $\underline{0}$ and $\underline{1}$ are the zero and unit matrix $(2 \times 2$ here). A ray tracing system for anisotropic media that corresponds to the system (2.5.11) can be derived yielding also Equation (2.6.2). In this case the matrix $\underline{\underline{\mathrm{S}}}$ is not given by Equation (2.6.3), but the following considerations also apply to anisotropic media.

A $4 \times 4$ matrix $\underline{=}$ is called an integral matrix, if it satisfies the relation

$$
\frac{\mathrm{d}}{\mathrm{d} s} \underline{\underline{\mathrm{A}}}=\underline{\underline{\mathrm{S}}} \underline{\underline{\mathrm{A}}}
$$


meaning that each column of $\underline{\underline{A}}$ obeys Equation (2.6.2). If $\underline{\underline{A}}=\underline{\underline{1}}$ for $s=s_{0}$, $\underline{\underline{A}}$ is also a propagator matrix (from $s_{0}$ ). If $\underline{\underline{A}}$ is an integral matrix formed by four linear independent solutions of (2.6.2), it is called a fundamental matrix.

The ray propagator matrix $\underline{\underline{\Pi}}\left(s, s_{0}\right)$ is a fundamental matrix formed as follows

$$
\underline{\underline{\Pi}}\left(s, s_{0}\right)=\left(\begin{array}{ll}
\underline{\mathrm{Q}}_{1}\left(s, s_{0}\right) & \underline{\mathrm{Q}}_{2}\left(s, s_{0}\right) \\
\underline{\mathrm{P}}_{1}\left(s, s_{0}\right) & \underline{\mathrm{P}}_{2}\left(s, s_{0}\right)
\end{array}\right),
$$

where $\underline{Q}_{1}, \underline{Q}_{2}, \underline{P}_{1}$ and $\underline{P}_{2}$ are $2 \times 2$ matrices with the following meaning: $\underline{Q}_{1}$ and $\underline{P}_{1}$ are solutions of the dynamic ray tracing Equations (2.5.17) for the initial conditions

$$
\underline{\mathrm{Q}}_{1}\left(s_{0}\right)=\underline{1} \text { and } \underline{\mathrm{P}}_{1}\left(s_{0}\right)=\underline{0},
$$

that describe a line source. $\underline{Q}_{2}$ and $\underline{P}_{2}$ also solve the dynamic ray tracing Equations (2.5.17), but for initial conditions of a point source

$$
\underline{\mathrm{Q}}_{2}\left(s_{0}\right)=\underline{0} \text { and } \underline{\mathrm{P}}_{2}\left(s_{0}\right)=\underline{1} \text {. }
$$

If $\underline{\underline{S}}$ is continuous, the ray propagator matrix satisfies the chain rule

$$
\underline{\underline{\Pi}}\left(s, s_{0}\right)=\underline{\underline{\Pi}}\left(s, s_{1}\right) \underline{\Pi}\left(s_{1}, s_{0}\right) \text {. }
$$

With

$$
\underline{\underline{\Pi}}\left(s_{0}, s_{0}\right)=\underline{\underline{\Pi}}\left(s_{0}, s_{1}\right) \underline{\underline{\Pi}}\left(s_{1}, s_{0}\right)=\underline{\underline{1}},
$$

it follows that

$$
\underline{\underline{\Pi}}^{-1}\left(s_{1}, s_{0}\right)=\underline{\underline{\Pi}}\left(s_{0}, s_{1}\right)
$$

which leads to

$$
\underline{\underline{\Pi}}^{-1}\left(s, s_{0}\right)=\left(\begin{array}{cc}
\underline{\mathrm{P}}_{2}^{\top}\left(s, s_{0}\right) & -\underline{\mathrm{Q}}_{2}^{\top}\left(s, s_{0}\right) \\
-\underline{\mathrm{P}}_{1}^{\top}\left(s, s_{0}\right) & \underline{\mathrm{Q}}_{1}^{\top}\left(s, s_{0}\right)
\end{array}\right) .
$$

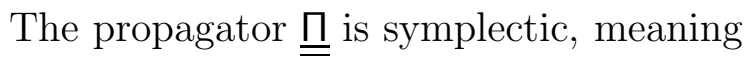

$$
\underline{\underline{\underline{n}}}^{\top} \underline{\underline{\underline{J}}} \underline{\underline{\underline{\Pi}}}=\underline{\underline{\mathrm{J}}}
$$

with the matrix

$$
\underline{\underline{\mathrm{J}}}=\left(\begin{array}{rr}
\underline{0} & \underline{1} \\
-\underline{1} & \underline{0}
\end{array}\right) \text {. }
$$

This property yields the following relations

$$
\begin{aligned}
& \underline{\mathrm{Q}}_{1}^{\top} \underline{\mathrm{P}}_{1}-\underline{\mathrm{P}}_{1}^{\top} \underline{\mathrm{Q}}_{1}=\underline{0} \\
& \underline{\mathrm{Q}}_{2}^{\top} \underline{\mathrm{P}}_{2}-\underline{\mathrm{P}}_{2}^{\top} \underline{\mathrm{Q}}_{2}=\underline{0} \\
& \underline{\mathrm{P}}_{2}^{\top} \underline{\mathrm{Q}}_{1}-\underline{\mathrm{Q}}_{2}^{\top} \underline{\mathrm{P}}_{1}=\underline{1} \\
& \underline{\mathrm{Q}}_{1}^{\top} \underline{\mathrm{P}}_{2}-\underline{\mathrm{P}}_{1}^{\top} \underline{\mathrm{Q}}_{2}=\underline{1}
\end{aligned},
$$


With $\underline{\underline{\Pi}}\left(s, s_{0}\right)$ the solution of Equation (2.6.2) can be written as

$$
\underline{\mathbf{W}}(s)=\underline{\underline{\Pi}}\left(s, s_{0}\right) \underline{\mathbf{W}}\left(s_{0}\right)
$$

for any initial conditions given at $s_{0}$ represented by $\underline{\mathrm{W}}\left(s_{0}\right)$. A similar solution exists for a (2.6.2)-like equation for the $4 \times 2$ matrix $\underline{\mathrm{X}}$

$$
\underline{X}=\left(\frac{Q}{P}\right),
$$

which is not to be mistaken for the matrix $\underline{\hat{X}}$ defined in (2.3.8). The dynamic ray tracing system (2.5.17) can thus be rewritten as

$$
\frac{\mathrm{d}}{\mathrm{d} s} \underline{\mathrm{X}}=\underline{\underline{\mathrm{S}}} \underline{\mathrm{X}}
$$

and is solved by

$$
\underline{\mathrm{X}}(s)=\underline{\underline{\Pi}}\left(s, s_{0}\right) \underline{\mathrm{X}}\left(s_{0}\right)
$$

Literature for Section 2.6:

- Červený (1985),

- Červený (2001).

\subsection{Paraxial Traveltimes}

Using Equation (2.5.23), the traveltime for a point $\left(q_{1}, q_{2}, s\right)$ in a vicinity of a point $\left(0,0, s_{0}\right)$ (see Figure 2.4) can be written as

$$
\tau\left(q_{1}, q_{2}, s\right)=\tau\left(s_{0}\right)+\left.\frac{\partial \tau}{\partial s}\right|_{s_{0}}\left(s-s_{0}\right)+\left.\frac{1}{2} \frac{\partial^{2} \tau}{\partial s^{2}}\right|_{s_{0}}\left(s-s_{0}\right)^{2}+\frac{1}{2} M_{I J}\left(s_{0}\right) q_{I} q_{J} \quad .
$$

The $s$-derivatives are evaluated with (2.5.25). Keeping only terms up to first order leads to

$$
\left.\frac{\partial \tau}{\partial s}\right|_{s_{0}}=\left.\frac{1}{V_{0}} \Rightarrow \frac{\partial^{2} \tau}{\partial s^{2}}\right|_{s_{0}}=-\left.\frac{1}{V_{0}^{2}} \frac{\partial V}{\partial s}\right|_{s_{0}}
$$

where $V_{0}=V\left(s_{0}\right)$. These expressions are inserted into (2.7.1) yielding

$$
\tau\left(q_{1}, q_{2}, s\right)=\tau\left(s_{0}\right)+\frac{1}{V_{0}}\left(s-s_{0}\right)-\left.\frac{1}{2 V_{0}^{2}} \frac{\partial V}{\partial s}\right|_{s_{0}}\left(s-s_{0}\right)^{2}+\frac{1}{2} M_{I J}\left(s_{0}\right) q_{I} q_{J} .
$$

The traveltime for a paraxial ray can also be expressed in local Cartesian coordinates $\hat{\boldsymbol{y}}$ with $y_{I}=q_{I}$ and $y_{3}=h\left(s-s_{0}\right)$. Introducing the $3 \times 3$ matrix $\underline{\hat{M}}$

$$
\underline{\hat{\mathbf{M}}}=\left(\begin{array}{ccc}
M_{11} & M_{12} & -\left.\frac{1}{V_{0}^{2}} \frac{\partial V}{\partial y_{1}}\right|_{s_{0}} \\
M_{21} & M_{22} & -\left.\frac{1}{V_{0}^{2}} \frac{\partial V}{\partial y_{2}}\right|_{s_{0}} \\
-\left.\frac{1}{V_{0}^{2}} \frac{\partial V}{\partial y_{1}}\right|_{s_{0}} & -\left.\frac{1}{V_{0}^{2}} \frac{\partial V}{\partial y_{2}}\right|_{s_{0}} & -\left.\frac{1}{V_{0}^{2}} \frac{\partial V}{\partial y_{3}}\right|_{s_{0}}
\end{array}\right)
$$


and the slowness vector in local Cartesian coordinates

$$
\hat{\boldsymbol{p}}^{(y)}\left(s_{0}\right)=\left(\begin{array}{c}
0 \\
0 \\
\frac{1}{V_{0}}
\end{array}\right),
$$

the paraxial traveltime becomes

$$
\tau\left(y_{i}\right)=\tau\left(s_{0}\right)+p_{i}^{(y)}\left(s_{0}\right) y_{i}+\frac{1}{2} M_{i j} y_{i} y_{j}
$$

To determine the slowness vector $\hat{\boldsymbol{p}}^{(y)}(s)$, the gradient of (2.7.6) with respect to $y$ is taken:

$$
p_{i}^{(y)}(s)=p_{i}^{(y)}\left(s_{0}\right)+M_{i j} y_{j}
$$

This substituted in (2.7.6) yields another expression for $\tau\left(y_{i}\right)$ :

$$
\tau\left(y_{i}\right)=\tau\left(s_{0}\right)+\frac{1}{2}\left(p_{i}^{(y)}(s)+p_{i}^{(y)}\left(s_{0}\right)\right) y_{i} .
$$

In general Cartesian coordinates $\hat{\boldsymbol{x}}$ Equation (2.7.6) becomes

$$
\tau\left(x_{i}\right)=\tau\left(s_{0}\right)+p_{i}^{(x)}\left(s_{0}\right) x_{i}+\frac{1}{2} H_{i k} M_{k l} H_{j l} x_{i} x_{j} \quad .
$$

Suppose now that the propagator matrix $\underline{\underline{\Pi}}\left(r_{0}, s_{0}\right)$ for a ray from $s_{0}$ to $r_{0}$ be known (see Figure 2.4). Let the slowness vectors $\boldsymbol{p}^{(q)}$ at $\boldsymbol{q}^{(r)}$ and $\boldsymbol{q}^{(s)}$ be denoted by the corresponding index. Then the relationship between $\boldsymbol{q}^{(r)}, \boldsymbol{p}^{(r)}, \boldsymbol{q}^{(s)}$ and $\boldsymbol{p}^{(s)}$ is given by (2.6.15):

$$
\begin{gathered}
\boldsymbol{q}^{(r)}=\underline{\mathrm{Q}}_{1}\left(r_{0}, s_{0}\right) \boldsymbol{q}^{(s)}+\underline{\mathrm{Q}}_{2}\left(r_{0}, s_{0}\right) \boldsymbol{p}^{(s)} \\
\boldsymbol{p}^{(r)}=\underline{\mathrm{P}}_{1}\left(r_{0}, s_{0}\right) \boldsymbol{q}^{(s)}+\underline{\mathrm{P}}_{2}\left(r_{0}, s_{0}\right) \boldsymbol{p}^{(s)} .
\end{gathered}
$$

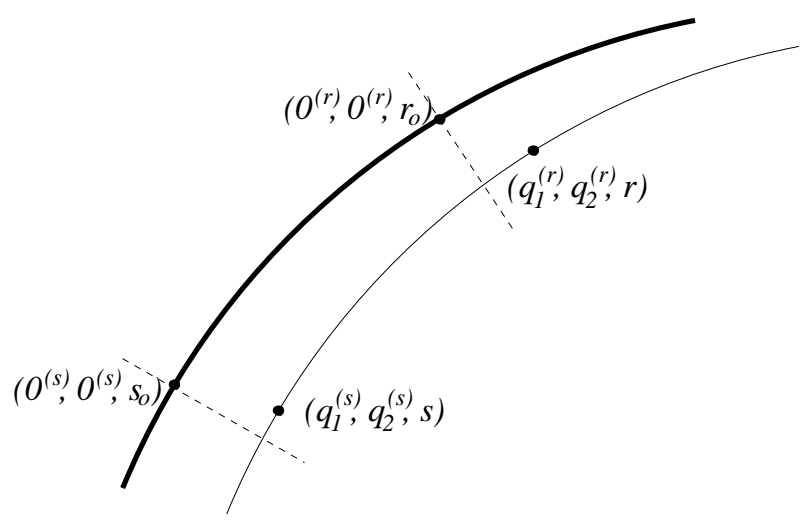

Figure 2.4: Central ray (bold) and a paraxial ray. The dashed lines indicate the wavefronts. 
Using (2.6.14) and $\underline{M}=\underline{P}_{2} \underline{Q}_{2}^{-1}$ for a point source, these equations can be solved for the slownesses, yielding

$$
\begin{aligned}
& \boldsymbol{p}^{(s)}=\underline{\mathrm{Q}}_{2}^{-1}\left(r_{0}, s_{0}\right) \boldsymbol{q}^{(r)}+\underline{\mathrm{M}}\left(s_{0}, r_{0}\right) \boldsymbol{q}^{(s)}, \\
& \boldsymbol{p}^{(r)}=\underline{\mathrm{M}}\left(r_{0}, s_{0}\right) \boldsymbol{q}^{(r)}+\underline{\mathrm{Q}}_{2}^{-\top}\left(r_{0}, s_{0}\right) \boldsymbol{q}^{(s)} .
\end{aligned}
$$

With these expressions for the slownesses in ray centred coordinates, the according formulations in local Cartesian coordinates are

$$
\begin{aligned}
\hat{\boldsymbol{p}}^{(y)}(s) & =\hat{\boldsymbol{p}}^{(y)}\left(s_{0}\right)+\underline{\mathrm{Q}}_{2}^{-1}\left(r_{0}, s_{0}\right) \boldsymbol{y}(r)+\underline{\mathrm{M}}\left(s_{0}, r_{0}\right) \boldsymbol{y}(s)-\left.\frac{1}{V_{0}^{2}} \frac{\partial c}{\partial y_{i}}\right|_{s_{0}} y_{i} \\
& =\hat{\boldsymbol{p}}^{(y)}\left(s_{0}\right)+\underline{\mathrm{Q}}_{2}^{-1}\left(r_{0}, s_{0}\right) \boldsymbol{y}(r)+\underline{\hat{\mathrm{M}}}\left(s_{0}, r_{0}\right) \hat{\boldsymbol{y}}(s) \\
\hat{\boldsymbol{p}}^{(y)}(r) & =\hat{\boldsymbol{p}}^{(y)}\left(r_{0}\right)-\underline{\mathrm{Q}}_{2}^{-\top}\left(r_{0}, s_{0}\right) \boldsymbol{y}(s)+\underline{\hat{\mathrm{M}}}\left(r_{0}, s_{0}\right) \hat{\boldsymbol{y}}(r) .
\end{aligned}
$$

Now $\tau(r, s)=\tau(r)-\tau(s)$ is built by using Equation (2.7.8)for $\tau(r)$ and $\tau(s)$ and inserting (2.7.12). This leads to

$$
\begin{aligned}
\tau(r, s)= & \tau\left(r_{0}\right)-\tau\left(s_{0}\right)+\hat{\boldsymbol{p}}^{(y)}\left(r_{0}\right) \cdot \hat{\boldsymbol{y}}(r)-\hat{\boldsymbol{p}}^{(y)}\left(s_{0}\right) \cdot \hat{\boldsymbol{y}}(s) \\
& +\frac{1}{2} \hat{\boldsymbol{y}}(r) \cdot \underline{\hat{\mathrm{M}}}\left(r_{0}, s_{0}\right) \hat{\boldsymbol{y}}(r)-\frac{1}{2} \hat{\boldsymbol{y}}(s) \cdot \underline{\hat{\mathrm{M}}}\left(s_{0}, r_{0}\right) \hat{\boldsymbol{y}}(s) \\
& -\frac{1}{2}\left[\hat{\boldsymbol{y}}(r) \cdot \underline{\mathrm{Q}}_{2}^{-\top} \hat{\boldsymbol{y}}(s)+\hat{\boldsymbol{y}}(s) \cdot \underline{\mathrm{Q}}_{2}^{-} \hat{\boldsymbol{y}}(r)\right],
\end{aligned}
$$

introducing the matrix $\underline{\mathrm{Q}}_{2}^{-}$and its transposed $\underline{\mathrm{Q}}_{2}^{-\top}$ such that $\underline{\mathrm{Q}}_{2}^{-1}$ is the upper left submatrix of $\underline{\mathbf{Q}}_{2}^{-}$and the elements $\underline{\hat{Q}}_{2_{i 3}^{-}}^{-}$and $\underline{\hat{Q}}_{23 i}^{-}$are zero. With

$$
\hat{\boldsymbol{y}}(r) \cdot \underline{\mathrm{Q}}_{2}^{-\top} \hat{\boldsymbol{y}}(s)=\hat{\boldsymbol{y}}(s) \cdot \underline{\mathrm{Q}}_{2}^{-} \hat{\boldsymbol{y}}(r)
$$

and $\tau_{0}=\tau\left(r_{0}\right)-\tau\left(s_{0}\right)$ the final result for the paraxial traveltime from $s$ to $r$ in local Cartesian coordinates becomes

$$
\begin{aligned}
\tau(r, s)= & \tau_{0}+\hat{\boldsymbol{p}}^{(y)}\left(r_{0}\right) \cdot \hat{\boldsymbol{y}}(r)-\hat{\boldsymbol{p}}^{(y)}\left(s_{0}\right) \cdot \hat{\boldsymbol{y}}(s)-\hat{\boldsymbol{y}}(s) \cdot \underline{\mathrm{Q}}_{2}^{-} \hat{\boldsymbol{y}}(r) \\
& +\frac{1}{2} \hat{\boldsymbol{y}}(r) \cdot \underline{\hat{\mathrm{M}}}\left(r_{0}, s_{0}\right) \hat{\boldsymbol{y}}(r)-\frac{1}{2} \hat{\boldsymbol{y}}(s) \cdot \underline{\hat{\mathrm{M}}}\left(s_{0}, r_{0}\right) \hat{\boldsymbol{y}}(s) .
\end{aligned}
$$

Again, this can also be written in general Cartesian coordinates $\hat{\boldsymbol{x}}$ :

$$
\begin{aligned}
\tau(r, s)= & \tau_{0}+\hat{\boldsymbol{p}}^{(x)}\left(r_{0}\right) \cdot \hat{\boldsymbol{x}}(r)-\hat{\boldsymbol{p}}^{(x)}\left(s_{0}\right) \cdot \hat{\boldsymbol{x}}(s)-\hat{\boldsymbol{x}}(s) \cdot \underline{\hat{\mathrm{H}}}\left(s_{0}\right) \underline{\mathrm{Q}}_{2}^{-} \underline{\hat{\mathrm{H}}}^{\top}\left(r_{0}\right) \hat{\boldsymbol{x}}(r) \\
& +\frac{1}{2} \hat{\boldsymbol{x}}(r) \cdot \underline{\hat{\mathrm{H}}}\left(r_{0}\right) \underline{\hat{\mathrm{M}}}\left(r_{0}, s_{0}\right) \underline{\hat{\mathrm{H}}}^{\top}\left(r_{0}\right) \hat{\boldsymbol{x}}(r) \\
& -\frac{1}{2} \hat{\boldsymbol{x}}(s) \cdot \underline{\hat{\mathrm{H}}}\left(s_{0}\right) \underline{\hat{\mathrm{M}}}\left(s_{0}, r_{0}\right) \underline{\hat{\mathrm{H}}}^{\top}\left(s_{0}\right) \hat{\boldsymbol{x}}(s) .
\end{aligned}
$$

Although isotropic equations were used for the derivation of the resulting traveltime expressions, they are also valid in anisotropic media, provided that the corresponding anisotropic matrices $\underline{\hat{M}}$ and $\underline{Q}_{2}$ are used, see Červený (2001).

Literature for Section 2.7:

- Červený (1985),

- Červený (2001). 


\subsection{Interfaces}

Until now velocities were assumed to be continuous functions with continuous first and second derivatives. This section describes the influence of a smooth curved interface, where the velocity has a discontinuity. If a ray arrives at a boundary between two different media, it will undergo an abrupt change in direction. This results in a discontinuity also in the matrix $\underline{\underline{M}}$. The purpose of this section is to determine the transformation of slowness vectors and matrices $\underline{\hat{M}}$ over interfaces.

Consider the traveltimes of incoming and outgoing (meaning reflected or transmitted) waves described by (2.7.6) in local Cartesian coordinates, where the point $s_{0}=b$ lies on the boundary. Since the direction of the ray changes on impinging on the boundary, the base vectors of the ray centred coordinates associated with the incoming and outgoing wave do not coincide. Therefore, yet another set of Cartesian coordinates $\hat{z}$ is introduced, that is associated with the interface. Its base vectors $\overrightarrow{\boldsymbol{\imath}}_{i}$ are defined as follows (see Figure $2.5)$ :

1. The normal to the interface corresponds to $\overrightarrow{\boldsymbol{\imath}}_{3}$. It points into the medium of the incident wave, meaning that $\hat{\boldsymbol{p}} \cdot \overrightarrow{\boldsymbol{z}}_{3}<0$.

2. The base vector $\overrightarrow{\boldsymbol{\imath}}_{1}$ is oriented along the intersection of the interfaces tangent plane with the plane of incidence. Its direction is such that $\hat{\boldsymbol{p}} \cdot \overrightarrow{\boldsymbol{\imath}}_{1}>0$.

3. Vector $\overrightarrow{\boldsymbol{\imath}}_{2}$ is tangent vector to the interface and perpendicular to the plane of incidence, $\overrightarrow{\boldsymbol{\imath}}_{2}=\overrightarrow{\boldsymbol{\imath}}_{3} \times \overrightarrow{\boldsymbol{\imath}}_{1}$.

The case of vertical incidence is not considered. Note that different directions are used throughout the literature. The transformation between this interface Cartesian coordinate system and the general Cartesian system is described by the matrix $\underline{\underline{Z}}$ with

$$
Z_{i j}=\frac{\partial x_{i}}{\partial z_{j}}=\frac{\partial z_{j}}{\partial x_{i}}
$$

and the transformation from ray centred coordinates to interface Cartesian coordinates by a matrix $\underline{\underline{G}}$. This matrix can be expressed in two fashions, first as a product of the transformation matrices $\underline{\hat{Z}}$ and $\underline{\hat{H}}$

$$
\hat{\underline{G}}=\hat{Z}^{\top} \underline{\hat{H}}=\left(\begin{array}{ccc}
\overrightarrow{\boldsymbol{e}}_{1} \cdot \overrightarrow{\boldsymbol{\imath}}_{1} & \overrightarrow{\boldsymbol{e}}_{2} \cdot \overrightarrow{\boldsymbol{\imath}}_{1} & \overrightarrow{\boldsymbol{t}} \cdot \overrightarrow{\boldsymbol{\imath}}_{1} \\
\overrightarrow{\boldsymbol{e}}_{1} \cdot \overrightarrow{\boldsymbol{\imath}}_{2} & \overrightarrow{\boldsymbol{e}}_{2} \cdot \overrightarrow{\boldsymbol{\imath}}_{2} & 0 \\
\overrightarrow{\boldsymbol{e}}_{1} \cdot \overrightarrow{\boldsymbol{\imath}}_{3} & \overrightarrow{\boldsymbol{e}}_{2} \cdot \overrightarrow{\boldsymbol{\imath}}_{3} & \overrightarrow{\boldsymbol{t}} \cdot \overrightarrow{\boldsymbol{\imath}}_{3}
\end{array}\right)
$$

The matrix $\underline{\hat{G}}=\underline{\hat{G}}^{\|} \underline{\hat{G}}^{\perp}$ can also be expressed by the two rotation matrices $\underline{\hat{G}}^{\|}$and $\underline{\hat{G}}^{\perp}$, where $\theta$ is the incidence angle between $\overrightarrow{\boldsymbol{\imath}}_{3}$ and $\overrightarrow{\boldsymbol{t}}$, and $\phi$ the angle between $\overrightarrow{\boldsymbol{\imath}}_{2}$ and $\overrightarrow{\boldsymbol{e}}_{2}$

$$
\underline{\mathrm{G}}^{\|}=\left(\begin{array}{ccc}
\cos \theta & 0 & \sin \theta \\
0 & 1 & 0 \\
-\sin \theta & 0 & \cos \theta
\end{array}\right) \quad \text { and } \quad \underline{\mathrm{G}}^{\perp}=\left(\begin{array}{ccc}
\cos \phi & -\sin \phi & 0 \\
\sin \phi & \cos \phi & 0 \\
0 & 0 & 1
\end{array}\right) \text {. }
$$


The traveltime expansion (2.7.9) expressed in interface coordinates is

$$
\tau\left(z_{i}\right)=\tau_{0}+p_{i}^{(x)} Z_{i j} z_{j}+\frac{1}{2} G_{i k} M_{k l} G_{j l} z_{i} z_{j}
$$

Now, let the 3-component of a vector $\hat{z}$ be described by

$$
z_{3}=\frac{1}{2} z_{I} D_{I J} z_{J}
$$

with $\underline{D}$ being the curvature matrix of the interface. Inserting this into Equation (2.8.4) and neglecting terms of higher order than two yields

$$
\tau\left(z_{I}\right)=\tau_{0}+p_{i}^{(x)} Z_{i J} z_{J}+\frac{1}{2} F_{I J} z_{I} z_{J}
$$

The matrix $\underline{\mathrm{F}}$ is given by

$$
\underline{\mathrm{F}}=\underline{\mathrm{G}} \underline{\mathrm{M}} \underline{\mathrm{G}}^{\top}+\frac{\cos \theta}{V} \underline{\mathrm{D}}+\underline{\mathrm{E}}
$$

where

$$
\begin{aligned}
E_{I J} & =G_{I 3} G_{J K} M_{3 K}+G_{I 3} G_{J 3} M_{33}+G_{I K} G_{J 3} M_{K 3} \\
& =-\frac{1}{V^{2}}\left(\left.G_{I 3} G_{J K} \frac{\partial V}{\partial q_{K}}\right|_{b}+\left.G_{I 3} G_{J 3} \frac{\partial V}{\partial q_{3}}\right|_{b}+\left.G_{I K} G_{J 3} \frac{\partial V}{\partial q_{K}}\right|_{b}\right)
\end{aligned}
$$

and the matrix $\underline{G}$ is the upper left $(2 \times 2)$ submatrix of $\underline{\hat{G}}$

$$
\underline{G}=\left(\begin{array}{cc}
\cos \theta \cos \phi & -\cos \theta \sin \phi \\
\sin \phi & \cos \phi
\end{array}\right) \text {. }
$$

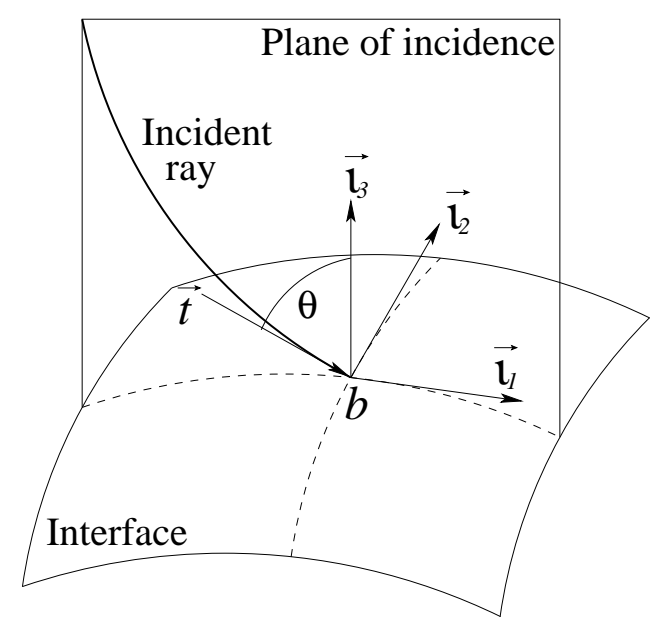

Figure 2.5: Definition of the Cartesian coordinate system associated with an interface: $\overrightarrow{\boldsymbol{\imath}}_{3}$ is normal to the interface, $\overrightarrow{\boldsymbol{\imath}}_{1}$ lies in the incidence plane and the interface tangent plane at $b$, and $\overrightarrow{\boldsymbol{\imath}}_{2}$ is given by $\overrightarrow{\boldsymbol{\imath}}_{3} \times \overrightarrow{\boldsymbol{\imath}}_{1}$. 
The terms in the matrix $\underline{F}$ have simple physical meaning: the matrix $\underline{D}$ describes the geometry of the interface, i.e. the curvature. For a plane reflector $\underline{\mathrm{D}}$ vanishes. The matrix $\underline{E}$ describes the inhomogeneity of the medium. This term vanishes in a constant velocity medium.

Suppose that Equation (2.8.6) describes the traveltime of an incident wave at a point $b$ on the interface, where all quantities in (2.8.6) are given at $b$. A similar equation can be written for reflected/transmitted waves. To distinguish between incident and reflected/transmitted wave, parameters of the reflected/transmitted wave will be denoted by a tilde. Phase matching along the interface in a near vicinity of $b$ requires

$$
\tau+p_{i}^{(x)} Z_{i J} z_{J}+\frac{1}{2} F_{I J} z_{I} z_{J}=\tilde{\tau}+\tilde{p}_{i}^{(x)} Z_{i J} z_{J}+\frac{1}{2} \tilde{F}_{I J} z_{I} z_{J}
$$

If this is to be fulfilled for any $z_{I}$, it leads to

$$
\begin{aligned}
\tau & =\tilde{\tau} \\
p_{i}^{(x)} Z_{i J} & =\tilde{p}_{i}^{(x)} Z_{i J} \\
F_{I J} & =\tilde{F}_{I J} .
\end{aligned}
$$

The second relation yields Snell's law. The third relation

$$
\underline{\mathrm{G}} \underline{\mathrm{M}} \underline{\mathrm{G}}^{\top}+\frac{\cos \theta}{V} \underline{\mathrm{D}}+\underline{\mathrm{E}}=\underline{\tilde{\mathrm{G}}} \underline{\tilde{\mathrm{M}}} \underline{\mathrm{G}}^{\top}+\frac{\cos \tilde{\theta}}{\tilde{V}} \underline{\mathrm{D}}+\underline{\tilde{\mathrm{E}}}
$$

can be rewritten to an expression for the transformation of the matrix $\underline{M}$ across the interface

$$
\underline{\tilde{M}}=\underline{\tilde{G}}^{-1}\left[\underline{G} \underline{M} \underline{G}^{\top}+u \underline{D}+\underline{E}-\underline{\tilde{E}}\right] \underline{\tilde{G}}^{-\top}
$$

where the abbreviation

$$
u=\frac{\cos \theta}{V}-\frac{\cos \tilde{\theta}}{\tilde{V}}
$$

was used.

The following step, the transformation of the matrices $\underline{Q}$ and $\underline{P}$, is straightforward. Since the ray parameters $\gamma_{I}$ must not change for reflection or transmission, they can be matched as was done for the phases in (2.8.11)

$$
\left.\frac{\partial \gamma_{I}}{\partial z_{J}}\right|_{b}=\left.\left.\frac{\partial \gamma_{I}}{\partial q_{K}}\right|_{b} \frac{\partial q_{K}}{\partial z_{J}}\right|_{b}
$$

This relation must be the same for the incident and outgoing wave and therefore

$$
\underline{\mathrm{Q}}^{-1} \underline{\mathrm{G}}^{\top}=\underline{\tilde{\mathrm{Q}}}^{-1} \underline{\tilde{\mathrm{G}}}^{\top} \Rightarrow \underline{\tilde{\mathrm{Q}}}=\underline{\tilde{\mathrm{G}}}^{\top} \underline{\mathrm{G}}^{-\top} \underline{\mathrm{Q}} .
$$

For the geometrical spreading, this means that with $\operatorname{det} \underline{G}=\cos \theta$

$$
\operatorname{det} \underline{\tilde{Q}}=\frac{\cos \tilde{\theta}}{\cos \theta} \underline{Q}
$$


The corresponding relation for $\underline{\tilde{P}}$ follows from $(2.8 .13),(2.8 .16)$, and $\underline{M}=\underline{P} \underline{Q}^{-1}$

$$
\underline{\tilde{P}}=\underline{\tilde{G}}^{-1}\left[\underline{G} \underline{P}+(u \underline{D}+\underline{E}-\underline{\tilde{E}}) \underline{G}^{-\top} \underline{Q}\right] .
$$

Equations (2.8.16) and (2.8.18) can be summarised using the $4 \times 2$ matrix $\underline{\mathrm{X}}$ that was defined in (2.6.16)

$$
\underline{\tilde{X}}=\underline{F} \underline{X}
$$

by introducing a $4 \times 4$ interface matrix $\underline{\underline{F}}$ with

$$
\underline{\underline{F}}=\left(\begin{array}{ll}
\underline{\tilde{G}}^{\top} & \underline{0}^{-1} \\
\underline{\underline{G}} & \underline{\underline{G}}^{-1}
\end{array}\right)\left(\begin{array}{ll}
u \underline{D}+\underline{\underline{E}}-\underline{\tilde{E}} & \underline{1}
\end{array}\right)\left(\begin{array}{ll}
\underline{G}^{-\top} & \underline{0} \\
\underline{0} & \underline{G}
\end{array}\right) .
$$

Similar to (2.6.18), ray propagator matrices can be used to describe the ray from a starting point $b_{0}$ passing the interface at $b$ and arriving at $s$ by

$$
\underline{\mathrm{X}}(s)=\underline{\underline{\Pi}}(s, b) \underline{\mathrm{F}}(b) \underline{\underline{\Pi}}\left(b, b_{0}\right) \underline{\mathrm{X}}\left(b_{0}\right) \text {. }
$$

For the transformation of displacement amplitudes across the interface, the reflection/transmission coefficient needs to be included. This can be done in terms of the matrix $\underline{\hat{R}}$ of reflection/transmission coefficients, yielding

$$
\tilde{\hat{U}}=\underline{\hat{R}} \underline{\hat{G}}^{\perp} \hat{U}
$$

The 1-component of the vector $\hat{\boldsymbol{U}}$ corresponds to a P-wave (as defined in Section 2.2), the 2-component is an SH-wave and the 3-component an SV-wave. This means that, e.g., the 11-component of the matrix $\underline{\hat{R}}$ is the P-P reflection/transmission coefficient. The final expression for the displacement vector reads

$$
\hat{\boldsymbol{U}}(s)=\frac{1}{\sqrt{V(s) \rho(s) \operatorname{det} \underline{\mathrm{Q}}(s)}} \sqrt{\frac{\tilde{V}(b) \tilde{\rho}(b) \operatorname{det} \underline{\tilde{Q}}(b)}{V(b) \rho(b) \operatorname{det} \underline{\mathbf{Q}}(b)}} \hat{\hat{\mathrm{R}}}(b) \underline{\hat{\mathrm{G}}}^{\perp}(b) \hat{\mathbf{\Psi}}\left(b_{0}\right)
$$

If not only one but $N$ interfaces are involved, Equations (2.8.21) and (2.8.23) change as follows: the chain rule (2.6.8) for propagator matrices applied to (2.8.21) immediately leads to

$$
\underline{\mathrm{X}}(s)=\underline{\underline{\Pi}}\left(s, b_{N}\right) \prod_{i=N}^{1}\left[\underline{\underline{\mathrm{F}}}\left(b_{i}\right) \underline{\underline{\Pi}}\left(b_{i}, b_{i-1}\right)\right] \underline{\mathrm{X}}\left(b_{0}\right) \text {. }
$$

The according expression for the displacement becomes

$$
\hat{\boldsymbol{U}}(s)=\frac{\mathrm{e}^{i \delta \tau\left(s, b_{0}\right)}}{\sqrt{V(s) \rho(s) \operatorname{det} \underline{\mathrm{Q}}(s)}} \prod_{i=N}^{1} \sqrt{\frac{\tilde{V}\left(b_{i}\right) \tilde{\rho}\left(b_{i}\right) \operatorname{det} \underline{\tilde{\mathrm{Q}}}\left(b_{i}\right)}{V\left(b_{i}\right) \rho\left(b_{i}\right) \operatorname{det} \underline{\mathbf{Q}}\left(b_{i}\right)}} \prod_{i=N}^{1}\left[\underline{\hat{\mathrm{R}}}\left(b_{i}\right) \underline{\hat{\mathrm{G}}}^{\perp}\left(b_{i}\right)\right] \hat{\mathbf{\Psi}}\left(b_{0}\right)
$$

where $\delta \tau\left(s, b_{0}\right)=-\pi / 2 \kappa\left(s, b_{0}\right)$ is a phase shift due to caustics. The KMAH index $\kappa$ of the ray trajectory was introduced in (2.3.23). It is convenient to rewrite Equation (2.8.25) by 
introducing the following notations: let the scalar quantity $\mathcal{A}(s)$ cover the transmission losses

$$
\mathcal{A}(s)=\frac{1}{\sqrt{V(s) \rho(s)}} \prod_{i=N}^{1} \sqrt{\frac{\tilde{V}\left(b_{i}\right) \tilde{\rho}\left(b_{i}\right)}{V\left(b_{i}\right) \rho\left(b_{i}\right)}} .
$$

The reflection/transmission matrix $\underline{\hat{R}}$ is summarised by

$$
\underline{\hat{\mathrm{R}}}(s)=\prod_{i=N}^{1} \underline{\hat{\mathrm{R}}}\left(b_{i}\right) \underline{\hat{\mathrm{G}}}^{\perp}\left(b_{i}\right)
$$

and the relative geometrical spreading $L(s)$ is given by

$$
L(s)=\left|\operatorname{det} \underline{\mathrm{Q}}(s) \prod_{i=N}^{1} \frac{\cos \theta\left(b_{i}\right)}{\cos \tilde{\theta}\left(b_{i}\right)}\right|^{\frac{1}{2}} \mathrm{e}^{i \delta \tau\left(s, b_{0}\right)}=\left|\operatorname{det} \underline{\mathrm{Q}}(s) \prod_{i=N}^{1} \frac{\operatorname{det} \underline{\mathrm{Q}}\left(b_{i}\right)}{\operatorname{det} \underline{\tilde{Q}}\left(b_{i}\right)}\right|^{\frac{1}{2}} \mathrm{e}^{i \delta \tau\left(s, b_{0}\right)} .
$$

With these, Equation (2.8.25) becomes

$$
\hat{\boldsymbol{U}}(s)=\frac{\mathcal{A}(s)}{L(s)} \hat{\mathrm{R}}(s) \hat{\mathbf{\Psi}}\left(b_{0}\right)
$$

Literature for Section 2.8:

- Červený (1985),

- Červený (2001).

\subsection{The Bortfeld Propagator}

The propagator $\underline{\underline{I}}$ that was introduced by Bortfeld obeys similar relations as the $\underline{\underline{\Pi}}$ prop-

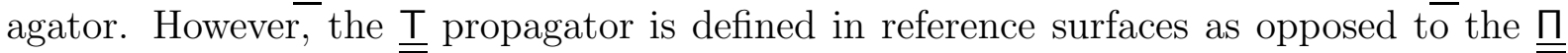
formalism which is based on an expansion into the wavefront. Consider a central ray that emerges from a point $s_{0}$ in a surface, the so-called anterior surface and arrives at a point $r_{0}$ in the posterior surface. Both surfaces have local Cartesian coordinate systems fixed with their origins at $s_{0}$ and $r_{0}$, respectively. Let the orientation of the systems be such that the 3 -component coincides with the normal to the surfaces at $s_{0}$ and $r_{0}$. The corresponding slowness vectors are

$$
\hat{\boldsymbol{p}}\left(s_{0}\right)=\frac{\overrightarrow{\boldsymbol{t}}\left(s_{0}\right)}{V\left(s_{0}\right)} \quad \text { and } \quad \hat{\boldsymbol{p}}\left(r_{0}\right)=\frac{\overrightarrow{\boldsymbol{t}}\left(r_{0}\right)}{V\left(r_{0}\right)} .
$$

A paraxial ray at a point $s$ has the coordinates

$$
\hat{\boldsymbol{x}}(s)=\left(x_{1}, x_{2}, f\left(x_{1}, x_{2}\right)\right)^{\top}
$$

and the slowness

$$
\hat{\boldsymbol{p}}(s)=\frac{\overrightarrow{\boldsymbol{t}}(s)}{V(s)}
$$




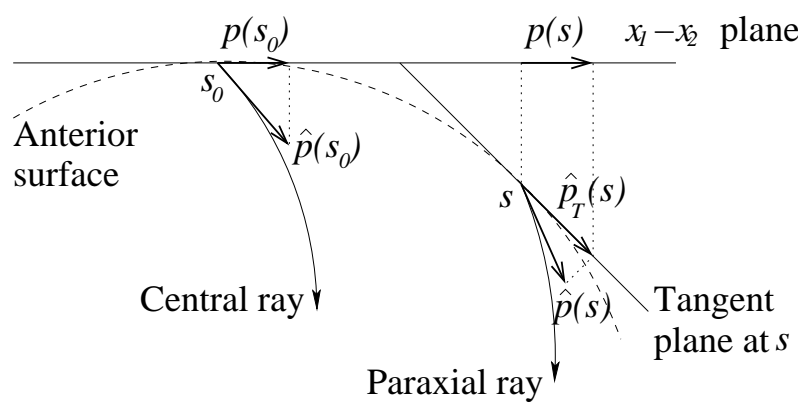

Figure 2.6: Projection of the slowness into the $x_{1}-x_{2}$ plane.

Points in a near vicinity of $s$ are described by $\hat{\boldsymbol{x}}+\mathrm{d} \hat{\boldsymbol{x}}$ with

$$
\mathrm{d} \hat{\boldsymbol{x}}=(\mathrm{d} \boldsymbol{x}, \vec{\nabla} f \cdot \mathrm{d} \boldsymbol{x})^{\top} .
$$

In the $x_{1}-x_{2}$ plane the 3 -D vectors $\hat{\boldsymbol{x}}(s)$ and $\hat{\boldsymbol{p}}(s)$ are represented by 2-D vectors $\boldsymbol{x}(s)$ and $\boldsymbol{p}(s)$. The vector $\boldsymbol{x}(s)$ is simply the projection of $\hat{\boldsymbol{x}}(s)$ onto the $x_{1}-x_{2}$ plane and was already used in Equation (2.9.4). To obtain the slowness vector $\boldsymbol{p}(s)$, two cascaded projections are required (see Figure 2.6): in the first step, $\hat{\boldsymbol{p}}(s)$ is projected onto the tangent plane of the anterior surface at $s$. This yields the tangential slowness vector $\hat{\boldsymbol{p}}_{T}(s)$ parallel to the tangent plane with

$$
\mathrm{d} \hat{\boldsymbol{p}}_{T}=(\mathrm{d} \boldsymbol{p}, \vec{\nabla} f \cdot \mathrm{d} \boldsymbol{p})^{\top} .
$$

The vector $\boldsymbol{p}(s)$ is the projection of $\hat{\boldsymbol{p}}_{T}(s)$ onto the $x_{1}-x_{2}$ plane. The same applies to the properties in the posterior surface.

The relationship between the initial vectors $\boldsymbol{x}(s)$ and $\boldsymbol{p}(s)$ and the final vectors $\boldsymbol{x}(r)$ and $\boldsymbol{p}(r)$ is expressed by a first order approximation that is valid in a near vicinity of the central ray

$$
\begin{aligned}
\boldsymbol{x}(r) & =\underline{\mathrm{A}} \boldsymbol{x}(s)+\underline{\mathrm{B}}\left(\boldsymbol{p}(s)-\boldsymbol{p}\left(s_{0}\right)\right), \\
\boldsymbol{p}(r)-\boldsymbol{p}\left(r_{0}\right) & =\underline{\mathrm{C}} \boldsymbol{x}(s)+\underline{\mathrm{D}}\left(\boldsymbol{p}(s)-\boldsymbol{p}\left(s_{0}\right)\right),
\end{aligned}
$$

where the $2 \times 2$ matrices are the derivatives

$$
\begin{aligned}
& A_{I J}=\left.\frac{\partial x(r)}{\partial x(s)}\right|_{r_{0}, s_{0}}, \quad B_{I J}=\left.\frac{\partial x(r)}{\partial p(s)}\right|_{r_{0}, s_{0}}, \\
& C_{I J}=\left.\frac{\partial p(r)}{\partial x(s)}\right|_{r_{0}, s_{0}} \quad, \quad D_{I J}=\left.\frac{\partial p(r)}{\partial p(s)}\right|_{r_{0}, s_{0}} .
\end{aligned}
$$

Since (2.9.6) bears a close similarity to $(2.7 .10),(2.9 .6)$ can be rewritten to

$$
\left(\begin{array}{c}
\boldsymbol{x}(r) \\
\boldsymbol{p}(r)-\boldsymbol{p}\left(r_{0}\right)
\end{array}\right)=\underline{\mathrm{T}}\left(r_{0}, s_{0}\right)\left(\begin{array}{c}
\boldsymbol{x}(s) \\
\boldsymbol{p}(s)-\boldsymbol{p}\left(s_{0}\right)
\end{array}\right),
$$

introducing the propagator matrix $\underline{\underline{\mathrm{T}}}\left(r_{0}, s_{0}\right)$

$$
\underline{\mathrm{T}}\left(r_{0}, s_{0}\right)=\left(\begin{array}{ll}
\underline{\mathrm{A}}\left(r_{0}, s_{0}\right) & \underline{\mathrm{B}}\left(r_{0}, s_{0}\right) \\
\underline{\mathrm{C}}\left(r_{0}, s_{0}\right) & \underline{\mathrm{D}}\left(r_{0}, s_{0}\right)
\end{array}\right) .
$$


The first order approximation of $\boldsymbol{x}$ and $\boldsymbol{p}$ corresponds to a second order approximation of the traveltime. The total differential of the traveltime is given by

$$
\begin{aligned}
\mathrm{d} \tau & =\vec{\nabla}_{r} \tau \cdot \mathrm{d} \hat{\boldsymbol{x}}(r)+\vec{\nabla}_{s} \tau \cdot \mathrm{d} \hat{\boldsymbol{x}}(s) \\
& =\hat{\boldsymbol{p}}(r) \cdot \mathrm{d} \hat{\boldsymbol{x}}(r)-\hat{\boldsymbol{p}}(s) \cdot \mathrm{d} \hat{\boldsymbol{x}}(s) \\
& =\hat{\boldsymbol{p}}_{T}(r) \cdot \mathrm{d} \hat{\boldsymbol{x}}(r)-\hat{\boldsymbol{p}}_{T}(s) \cdot \mathrm{d} \hat{\boldsymbol{x}}(s) \\
& =\left(\boldsymbol{p}(r), \vec{\nabla}_{r} f \cdot \boldsymbol{p}(r)\right) \cdot\left(\begin{array}{c}
\mathrm{d} \boldsymbol{x}(r) \\
\vec{\nabla}_{r} f \cdot \boldsymbol{x}(r)
\end{array}\right)-\left(\boldsymbol{p}(s), \vec{\nabla}_{s} f \cdot \boldsymbol{p}(s)\right) \cdot\left(\begin{array}{c}
\mathrm{d} \boldsymbol{x}(s) \\
\vec{\nabla}_{s} f \cdot \boldsymbol{x}(s)
\end{array}\right) \\
& \approx \boldsymbol{p}(r) \cdot \mathrm{d} \boldsymbol{x}(r)-\boldsymbol{p}(s) \cdot \mathrm{d} \boldsymbol{x}(s) .
\end{aligned}
$$

The last approximation is motivated by the aim for a second order approximation of $\tau$, therefore terms containing products of $f$ derivatives are neglected. The signs were chosen in a way that the traveltime increases for growing distance in the direction of propagation of the ray. Now $\boldsymbol{p}(r)$ and $\boldsymbol{p}(s)$ are expressed in terms of the $\underline{\underline{T}}$-submatrices from (2.9.6)

$$
\begin{aligned}
& \boldsymbol{p}(s)=\boldsymbol{p}\left(s_{0}\right)-\underline{\mathrm{B}}^{-1} \underline{\mathrm{A}} \boldsymbol{x}(s)+\underline{\mathrm{B}}^{-1} \boldsymbol{x}(r), \\
& \boldsymbol{p}(r)=\boldsymbol{p}\left(r_{0}\right)+\underline{\mathrm{DB}}^{-1} \boldsymbol{x}(r)+\left[\underline{\mathrm{C}}-\underline{\mathrm{DB}}^{-1} \underline{\mathrm{A}}\right] \boldsymbol{x}(s) .
\end{aligned}
$$

Inserting these slownesses into Equation (2.9.10), the latter can be integrated, leading to

$$
\begin{aligned}
\tau= & \tau_{0}+\boldsymbol{p}\left(r_{0}\right) \cdot \boldsymbol{x}(r)-\boldsymbol{p}\left(s_{0}\right) \cdot \boldsymbol{x}(s)-\boldsymbol{x}(s) \cdot \underline{\mathrm{B}}^{-1} \boldsymbol{x}(r) \\
& +\frac{1}{2} \boldsymbol{x}(s) \cdot \underline{\mathrm{B}}^{-1} \underline{\mathrm{A}} \boldsymbol{x}(s)+\frac{1}{2} \boldsymbol{x}(r) \cdot \underline{\mathrm{D}}^{-1} \boldsymbol{x}(r)
\end{aligned}
$$

$\left(\tau_{0}=\tau\left(s_{0}, r_{0}\right)\right)$. This requires that

$$
\underline{\mathrm{B}}^{-1} \underline{\mathrm{A}}=\underline{\mathrm{A}}^{\top} \underline{\mathrm{B}}^{-\top}, \quad \underline{\mathrm{D}}^{\top} \underline{\mathrm{B}}^{-1}=\underline{\mathrm{B}}^{-\top} \underline{\mathrm{D}}^{\top}, \quad \underline{\mathrm{A}}{ }^{\top} \underline{\mathrm{D}}-\underline{\mathrm{C}}^{\top} \underline{\mathrm{B}}=\underline{1},
$$

following from the demand that the order of differentiation of $\tau$ must be arbitrary. These relations yield the symplecticity of $\stackrel{T}{=}$ and

$$
\underline{B}^{\top} \underline{D}=\underline{D}^{\top} \underline{B}, \quad \underline{A}^{\top} \underline{C}=\underline{C}^{\top} \underline{A} .
$$

As for the propagator matrix $\underline{\underline{\underline{n}}}$, the inverse can be computed for $\underline{\underline{T}}$

$$
\underline{\underline{\mathrm{T}}}^{-1}\left(r_{0}, s_{0}\right)=\underline{\mathrm{T}}\left(s_{0}, r_{0}\right)=\left(\begin{array}{rr}
\underline{\mathrm{D}}\left(r_{0}, s_{0}\right)^{\top} & -\underline{\mathrm{B}}\left(r_{0}, s_{0}\right)^{\top} \\
-\underline{\mathrm{C}}\left(r_{0}, s_{0}\right)^{\top} & \underline{\mathrm{A}}\left(r_{0}, s_{0}\right)^{\top}
\end{array}\right) \text {. }
$$

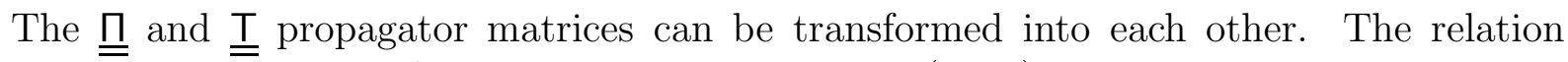
between them can be found by applying Equation (2.7.9) to the anterior and posterior surfaces, leading to

$$
\begin{aligned}
\tau= & \tau_{0}+\boldsymbol{p}\left(r_{0}\right) \cdot \boldsymbol{x}(r)-\boldsymbol{p}\left(s_{0}\right) \cdot \boldsymbol{x}(s)-\boldsymbol{x}(s) \cdot \underline{\mathrm{G}}\left(s_{0}\right) \underline{\mathrm{Q}}_{2}^{-1} \underline{\mathrm{G}}^{\top}\left(r_{0}\right) \boldsymbol{x}(r) \\
& -\frac{1}{2} \boldsymbol{x}(s) \cdot \underline{\mathrm{G}}\left(s_{0}\right) \underline{\mathrm{F}}\left(s_{0}\right) \underline{\mathrm{G}}^{\top}\left(s_{0}\right) \boldsymbol{x}(s)+\frac{1}{2} \boldsymbol{x}(r) \cdot \underline{\mathrm{G}}\left(r_{0}\right) \underline{\mathrm{F}}\left(r_{0}\right) \underline{\mathrm{G}}^{\top}\left(r_{0}\right) \boldsymbol{x}(r) .
\end{aligned}
$$


Comparison of (2.9.13) and (2.9.17) leads to

$$
\underline{\underline{\mathrm{T}}}\left(r_{0}, s_{0}\right)=\underline{\underline{\mathrm{X}}}\left(r_{0}\right) \underline{\underline{\mathrm{G}}}\left(r_{0}\right) \underline{\underline{\Pi}}\left(r_{0}, s_{0}\right) \underline{\underline{\mathrm{G}}}^{\top}\left(s_{0}\right) \underline{\underline{\mathrm{X}}}^{\top}\left(s_{0}\right)
$$

with

$$
\underline{\underline{\mathrm{G}}}\left(r_{0}\right)=\left(\begin{array}{ll}
\underline{\mathrm{G}}^{-1}\left(r_{0}\right) & \underline{0} \\
\underline{\underline{G}} & \underline{\mathrm{G}}^{\top}\left(r_{0}\right)
\end{array}\right) \quad, \quad \underline{\underline{\mathrm{G}}}\left(s_{0}\right)=\left(\begin{array}{ll}
\underline{\mathrm{G}}^{\top}\left(s_{0}\right) & \underline{\underline{0}}^{-1}\left(s_{0}\right)
\end{array}\right),
$$

and

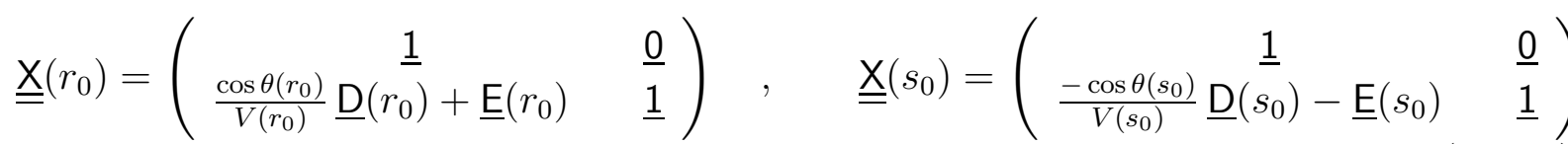

The matrices $\underline{\mathrm{D}}$ and $\underline{\mathrm{E}}$ are taken from (2.8.7) at $s_{0}$ and $r_{0}$ accordingly.

Literature for Section 2.9:

- Bortfeld (1989),

- Hubral et al. (1992a). 


\section{Chapter 3}

\section{Traveltime Interpolation}

Using finite difference (FD) eikonal solvers or the wavefront construction method (for an overview of both, see, e.g., Leidenfrost et al. 1999), traveltime tables can be computed efficiently. This is one foundation for the summation stack along diffraction surfaces for a Kirchhoff type migration. For a 3-D depth migration, however, large amounts of fine gridded traveltime maps are required. The effort in computational time as well as in data storage can be significantly reduced if a fast and accurate traveltime interpolation routine can be applied. Then only few original traveltime tables must be computed and stored on coarse grids, whereas fast interpolation is carried out onto a finer grid during migration of data.

In 1982, Ursin introduced a hyperbolic approximation for reflection traveltimes where the wavefront curvature matrix is determined by dynamic ray tracing. Gjøystdal et al. (1984) compared parabolic and hyperbolic traveltime approximations for a variety of general inhomogeneous 3-D models with curved interfaces, also employing dynamic ray tracing. They concluded that a hyperbolic traveltime approximation is more accurate than a parabolic one. A general second order approximation of traveltimes in seismic systems was established by Bortfeld (1989). His work is based on the paraxial ray approximation (Červený, 2001) and can be used to interpolate traveltimes for sources and receivers which are located in the bordering surfaces of the seismic system. Schleicher et al. (1993b) link the Bortfeld theory to the ray propagator formalism and introduce a hyperbolic variant of the paraxial traveltime interpolation. Both methods are, however, restricted to source and receiver reference surfaces.

Mendes (2000) suggests traveltime interpolation using the Dix hyperbolic equation. Since the Dix equation is only valid for horizontally layered media, this technique is not justified for other models. Brokešová (1996) states the superiority of the paraxial (parabolic) interpolation compared to linear and Fourier (sinc-) interpolation of traveltimes.

Gajewski (1998) not only finds the hyperbolic variant of the paraxial approximation far superior to trilinear interpolation but also introduces a technique to determine the interpolation coefficients directly from traveltimes, therefore providing a means to avoid dynamic ray tracing. That algorithm is, however, restricted to horizontal interpolation. 
Although the procedure can be repeated for vertical receiver lines, this does not allow for data reduction onto vertical coarse grids.

The traveltime interpolation algorithm that I present here is neither restricted to laterally homogeneous media nor to interpolation in reference surfaces respectively horizontal layers only. In contrast to most other methods, it also allows for the interpolation of sources, not only receivers. Since no assumptions are made on the velocities of the medium, the method can be applied to arbitrary 3-D heterogeneous and even anisotropic media.

Following the derivation of a parabolic and hyperbolic traveltime expansion in arbitrary 3-D media, I give a detailed description on the determination of the Taylor coefficients. In the following section I give examples for the coefficients in media where an analytic solution is available. They demonstrate that the coefficients can be determined from traveltimes with high accuracy. Application of the resulting traveltime interpolation to a variety of velocity models is shown in the next section. The models range from examples for which analytic solutions are known to a 3-D extension of the highly complex Marmousi model. The results of both parabolic and hyperbolic interpolation are compared to trilinear interpolation. As expected the hyperbolic variant is superior to the parabolic one. This is not surprising because it confirms that any wavefront can locally be approximated by a sphere. Also, the accuracy of second order interpolation is far better than trilinear interpolation, because the latter neglects the curvature of the wavefront. The required amount of computational time is of the same order for all three interpolation methods. If traveltimes for only every tenth grid point are stored, savings of up to a factor of $10^{6}$ are possible with no significant loss in accuracy. The final sections investigate the influence of the size of the coarse grid spacing and the behaviour of the method in the vicinity of discontinuities in the traveltime data.

\subsection{Parabolic Traveltime Expansion}

The following considerations are based upon the existence of first and second order continuous spatial derivatives for the traveltime fields. Traveltimes that satisfy these conditions can be expanded into a Taylor series up to second degree. Provided that the distance to the expansion point is small, the Taylor series yields a good approximation for the original traveltime function. The size of the vicinity describing "small" distances depends on the scale of velocity variations in the input model.

For the 3-D case, the Taylor expansion has to be carried out in 6 variables: the 3 components of the source position vector $\hat{\boldsymbol{s}}=\left(s_{1}, s_{2}, s_{3}\right)^{\top}$ and those of the receiver position $\hat{\boldsymbol{g}}=\left(g_{1}, g_{2}, g_{3}\right)^{\top}$. The values of $\hat{\boldsymbol{s}}$ and $\hat{\boldsymbol{g}}$ at the expansion point are $\hat{\boldsymbol{s}}_{0}$ and $\hat{\boldsymbol{g}}_{0}$ with the traveltime $\tau_{0}$ from $\hat{\boldsymbol{s}}_{0}$ to $\hat{\boldsymbol{g}}_{0}$. The variations in source and receiver positions $\Delta \hat{\boldsymbol{s}}$ and $\Delta \hat{\boldsymbol{g}}$ are such that $\hat{\boldsymbol{s}}=\hat{\boldsymbol{s}}_{0}+\Delta \hat{\boldsymbol{s}}$ and $\hat{\boldsymbol{g}}=\hat{\boldsymbol{g}}_{0}+\Delta \hat{\boldsymbol{g}}$. The Taylor expansion for $\tau\left(\hat{\boldsymbol{s}}_{0}+\Delta \hat{\boldsymbol{s}}, \hat{\boldsymbol{g}}_{0}+\Delta \hat{\boldsymbol{g}}\right)$ 
up to second order written in index notation $(i, j=1,2,3)$ is

$$
\begin{aligned}
\tau\left(s_{i}, g_{i}\right)= & \tau_{0}+\left.\Delta s_{i} \frac{\partial \tau}{\partial s_{i}}\right|_{\hat{\boldsymbol{s}}_{0}, \hat{\boldsymbol{g}}_{0}}+\left.\Delta g_{i} \frac{\partial \tau}{\partial g_{i}}\right|_{\hat{\boldsymbol{s}}_{0}, \hat{\boldsymbol{g}}_{0}}+\left.\Delta s_{i} \Delta g_{j} \frac{\partial^{2} \tau}{\partial s_{i} \partial g_{j}}\right|_{\hat{\boldsymbol{s}}_{0}, \hat{\boldsymbol{g}}_{0}} \\
& +\left.\frac{1}{2} \Delta s_{i} \Delta s_{j} \frac{\partial^{2} \tau}{\partial s_{i} \partial s_{j}}\right|_{\hat{\boldsymbol{s}}_{0}, \hat{\boldsymbol{g}}_{0}}+\left.\frac{1}{2} \Delta g_{i} \Delta g_{j} \frac{\partial^{2} \tau}{\partial g_{i} \partial g_{j}}\right|_{\hat{\boldsymbol{s}}_{0}, \hat{\boldsymbol{g}}_{0}}+\mathcal{O}(3)
\end{aligned}
$$

(summation convention is applied). I introduce the following notation for the slowness vectors $\hat{\boldsymbol{p}}_{0}$ and $\hat{\boldsymbol{q}}_{0}$ at $\hat{\boldsymbol{s}}_{0}$ and $\hat{\boldsymbol{g}}_{0}$, respectively

$$
p_{i_{0}}=-\left.\frac{\partial \tau}{\partial s_{i}}\right|_{\hat{\boldsymbol{s}}_{0}, \hat{\boldsymbol{g}}_{0}}, \quad q_{i_{0}}=\left.\frac{\partial \tau}{\partial g_{i}}\right|_{\hat{\boldsymbol{s}}_{0}, \hat{\boldsymbol{g}}_{0}}
$$

and the second order derivative matrices $\underline{\hat{S}}, \underline{\hat{G}}$ and $\underline{\hat{N}}$ with

$$
\begin{aligned}
& S_{i j}=-\left.\frac{\partial^{2} \tau}{\partial s_{i} \partial s_{j}}\right|_{\hat{\boldsymbol{s}}_{0}, \hat{\boldsymbol{g}}_{0}}=S_{j i}, \\
& G_{i j}=\left.\frac{\partial^{2} \tau}{\partial g_{i} \partial g_{j}}\right|_{\hat{\boldsymbol{s}}_{0}, \hat{\boldsymbol{g}}_{0}}=G_{j i}, \\
& N_{i j}=-\left.\frac{\partial^{2} \tau}{\partial s_{i} \partial g_{j}}\right|_{\hat{\boldsymbol{s}}_{0}, \hat{\boldsymbol{g}}_{0}} \neq N_{j i} .
\end{aligned}
$$

With these the Taylor series (3.1.1) reads

$$
\begin{aligned}
\tau(\hat{\boldsymbol{s}}, \hat{\boldsymbol{g}})= & \tau_{0}-\hat{\boldsymbol{p}}_{0}^{\top} \Delta \hat{\boldsymbol{s}}+\hat{\boldsymbol{q}}_{0}^{\top} \Delta \hat{\boldsymbol{g}}-\Delta \hat{\boldsymbol{s}}^{\top} \underline{\hat{\mathbf{N}}} \Delta \hat{\boldsymbol{g}} \\
& -\frac{1}{2} \Delta \hat{\boldsymbol{s}}^{\top} \underline{\hat{\mathbf{S}}} \Delta \hat{\boldsymbol{s}}+\frac{1}{2} \Delta \hat{\boldsymbol{g}}^{\top} \underline{\hat{\mathrm{G}}} \Delta \hat{\boldsymbol{g}}+\mathcal{O}(3) .
\end{aligned}
$$

Equation (3.1.4) describes the parabolic traveltime expansion. The signs were chosen in a form, that for growing distance between source and receiver the traveltime (e.g., in a homogeneous medium) increases.

Equation (3.1.4) states that for small variations of $\Delta \hat{\boldsymbol{s}}$ and $\Delta \hat{\boldsymbol{g}}$, traveltimes can be approximated by a parabola with high accuracy. If the $\mathcal{O}(3)$ term is neglected, Equation (3.1.4) can be used for the determination of the coefficients. This requires that traveltimes for certain source-receiver combinations are given, which can be substituted in Equation (3.1.4) and then solved for the coefficients. The aim is to apply this interpolation to migration, where the seismic reflection data is stacked along diffraction traveltime surfaces. To compute these stacking surfaces traveltime tables need to be computed in any event. Therefore I assume that such data is available. Application of the traveltime interpolation will, however, reduce the need to compute these traveltime tables on fine grids. Before discussing the traveltime interpolation I will demonstrate how to obtain the coefficients from traveltimes sampled on a coarse grid. 
In the following, Cartesian grids for both source and receiver positions are used. Sources are located in the $x$ - $y$-plane with $x, y$, and $z$ corresponding to the indices 1,2 , and 3 . To determine the slowness vectors $\hat{\boldsymbol{p}}_{0}$ and $\hat{\boldsymbol{q}}_{0}$ as well as the second order derivative matrices $\underline{\hat{S}}, \underline{\hat{G}}$, and $\underline{\hat{N}}$, tables containing traveltimes from the source to each subsurface coarse grid point are required for nine different source positions: the central source at $\hat{\boldsymbol{s}}_{0}$ and eight surrounding source positions (for a 2-D model the number of additional sources reduces to two). These additional sources are placed on the $x$-y-grid with a distance to the central source that in my example coincides with the coarse grid spacing $\Delta x$ and $\Delta y$. Note that the method is neither restricted to cubical grids nor to equal grid spacing for sources and receivers.

To compute $q_{x_{0}}$ and $G_{x x}$ from the traveltime tables, only the traveltimes $\tau_{0}, \tau_{1}$, and $\tau_{2}$ are required, as shown in Figure 3.1. The traveltimes $\tau_{1}$ and $\tau_{2}$ are inserted into the parabolic expansion (3.1.4), respectively. Building the sum and the difference of the resulting expressions yields

\begin{aligned}$\tau_{1} & =\tau_{0}-q_{x_{0}} \Delta g_{x}+\frac{1}{2} G_{x x} \Delta g_{x}^{2} \\ \tau_{2} & =\tau_{0}+q_{x_{0}} \Delta g_{x}+\frac{1}{2} G_{x x} \Delta g_{x}^{2} \\$\hline Sum: $\quad \tau_{1}+\tau_{2} & =2 \tau_{0} \quad+G_{x x} \Delta g_{x}^{2} \\$ Difference: $\tau_{1}-\tau_{2} & =-2 q_{x_{0}} \Delta g_{x}\end{aligned}$.

This can be solved for $q_{x_{0}}$ and $G_{x x}$

$$
q_{x_{0}}=\frac{\tau_{2}-\tau_{1}}{2 \Delta g_{x}} \quad \text { and } \quad G_{x x}=\frac{\tau_{1}+\tau_{2}-2 \tau_{0}}{\Delta g_{x}^{2}} .
$$

Expressions for the remaining coefficients are given in Appendix D.

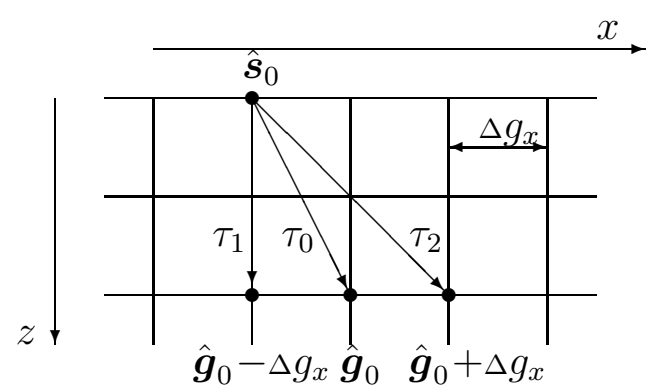

Figure 3.1: Determination of the coefficients $q_{x_{0}}$ and $G_{x x}$ : the traveltimes $\tau_{0}$ from $\hat{\boldsymbol{s}}_{0}$ to $\hat{\boldsymbol{g}}_{0}, \tau_{1}$ from $\hat{\boldsymbol{s}}_{0}$ to $\hat{\boldsymbol{g}}_{0}-\Delta g_{x}$, and $\tau_{2}$ from $\hat{\boldsymbol{s}}_{0}$ to $\hat{\boldsymbol{g}}_{0}+\Delta g_{x}$ are required.

The $y$ - and $z$-components of $\hat{\boldsymbol{q}}_{0}$ and $\underline{\underline{G}}$ can be found in the same way by varying $g_{y}$, respectively $g_{z}$. Varying both $g_{x}$ and $\bar{g}_{y}$ leads to $G_{x y} ; G_{y z}$ and $G_{z x}$ follow accordingly. 
The determination of the $x$ - and $y$-components of $\underline{\hat{S}}$ and $\hat{\boldsymbol{p}}_{0}$ is straightforward: instead of varying the receiver position, different source positions in the $x$ - $y$-surface are used. For the $x x$-, $y y$-, $x y$ - and $y x$-components of $\underline{\hat{N}}$, both source and receiver positions have to be varied in $x$ and $y$. The $z$-components of $\overline{\hat{p}}_{0}, \underline{\hat{S}}$, and $\underline{\hat{N}}$ can be determined from traveltimes for sources at different depths, following the same lines as for the $x$ - and $y$-components. For isotropic media, however, I propose a different approach without having to compute these additional traveltime tables for sources in the subsurface. The eikonal equation

$$
\left(\frac{\partial \tau}{\partial x}\right)^{2}+\left(\frac{\partial \tau}{\partial y}\right)^{2}+\left(\frac{\partial \tau}{\partial z}\right)^{2}=\frac{1}{V^{2}}
$$

can be solved for the $z$-component of the slowness vector $\hat{\boldsymbol{p}}$

$$
p_{z}=\sqrt{\frac{1}{V_{s}^{2}}-p_{x}^{2}-p_{y}^{2}} .
$$

This requires that the source lies in the top surface of the model, otherwise the sign of $p_{z}$ must be taken into account. This case is, however, not considered here. The velocity $V_{s}$ in (3.1.6) is the phase velocity at the source. With the definitions (3.1.2) and (3.1.3), $\underline{\hat{S}}$ and $\underline{\hat{N}}$ can be rewritten to

$$
\begin{aligned}
S_{i j} & =\left.\left(\frac{\partial p_{i}}{\partial s_{j}}\right)\right|_{\hat{\boldsymbol{s}}_{0}, \hat{\boldsymbol{g}}_{0}}, \\
N_{i j} & =-\left.\left(\frac{\partial q_{j}}{\partial s_{i}}\right)\right|_{\hat{\boldsymbol{s}}_{0}, \hat{\boldsymbol{g}}_{0}}=\left.\left(\frac{\partial p_{i}}{\partial g_{j}}\right)\right|_{\hat{\boldsymbol{s}}_{0}, \hat{\boldsymbol{g}}_{0}} .
\end{aligned}
$$

Substituting $p_{z}$ in Equations (3.1.8) and (3.1.9) by (3.1.7), the second order derivatives of $\tau$ with respect to $s_{z}$ can be determined from the already known $x$-y-matrix elements and derivatives of the velocity. If the velocity field under consideration is continuous, the velocity derivatives can be determined with a second order FD operator on the coarse grid. 
The results for the $z$ elements of $\underline{\hat{S}}$ and $\underline{\hat{N}}$ are as follows:

$$
\begin{aligned}
S_{x z} & =\frac{\partial p_{z}}{\partial s_{x}}=\frac{\partial}{\partial s_{x}} \sqrt{\frac{1}{V_{s}^{2}}-p_{x}^{2}-p_{y}^{2}} \\
& =\frac{1}{2 p_{z_{0}}} \frac{\partial}{\partial s_{x}}\left(\frac{1}{V_{s}^{2}}-p_{x}^{2}-p_{y}^{2}\right) \\
& =-\frac{1}{V_{s}^{3} p_{z_{0}}} \frac{\partial V_{s}}{\partial s_{x}}-\frac{p_{x_{0}}}{p_{z_{0}}} \frac{\partial p_{x}}{\partial s_{x}}-\frac{p_{y_{0}}}{p_{z_{0}}} \frac{\partial p_{y}}{\partial s_{x}} \\
& =-\frac{1}{V_{s}^{3} p_{z_{0}}} \frac{\partial V_{s}}{\partial s_{x}}-\frac{p_{x_{0}}}{p_{z_{0}}} S_{x x}-\frac{p_{y_{0}}}{p_{z_{0}}} S_{x y}=S_{z x} \\
S_{y z} & =-\frac{1}{V_{s}^{3} p_{z_{0}}} \frac{\partial V_{s}}{\partial s_{y}}-\frac{p_{x_{0}}}{p_{z_{0}}} S_{x y}-\frac{p_{y_{0}}}{p_{z_{0}}} S_{y y}=S_{z y} \\
S_{z z} & =-\frac{1}{V_{s}^{3} p_{z_{0}}} \frac{\partial V_{s}}{\partial s_{z}}-\frac{p_{x_{0}}}{p_{z_{0}}} S_{x z}-\frac{p_{y_{0}}}{p_{z_{0}}} S_{y z}, \\
N_{z x} & =-\frac{p_{x_{0}}}{p_{z_{0}}} N_{x x}-\frac{p_{y_{0}}}{p_{z_{0}}} N_{y x}, \\
N_{z y} & =-\frac{p_{x_{0}}}{p_{z_{0}}} N_{x y}-\frac{p_{y_{0}}}{p_{z_{0}}} N_{y y} \\
N_{z z} & =-\frac{1}{p_{z_{0}}}\left(p_{x_{0}} N_{x z}+p_{y_{0}} N_{y z}\right)
\end{aligned}
$$

(derivatives are taken at $\hat{\boldsymbol{s}}_{0}, \hat{\boldsymbol{g}}_{0}$ ). These expressions will not yield results if $p_{z_{0}}$ equals zero. This case has, however, no practical relevance for the applications that the method was developed for. Appendix D gives expressions for the determination of all coefficients from traveltimes.

For anisotropic media, the eikonal equation corresponds to Equation (3.1.6), but $V_{s}$ is a function of the phase normal (i.e. $V_{s}$ depends on the direction of wave propagation). To obtain the appropriate derivatives, results of Gajewski (1993) can be used. Until these formulae exist the anisotropic coefficients can be determined from traveltimes, however, for the derivatives with respect to the $z$ component of the source position traveltime tables for sources at different depths are required. Then these derivatives can be computed in the same way as the derivatives with respect to other components of the source and receiver position, as shown above. All expressions are given in Appendix D. The extension of the method to anisotropic media will also be considered in Chapter 10.

\subsection{Hyperbolic Traveltime Expansion}

Considering the simplest medium that we can think of, a homogeneous medium with constant velocity, it appears that there might be an even better approximation than the parabolic one: in a constant velocity medium the wavefronts are circles that translate into hyperbolic traveltimes. Therefore, it should be possible to locally approximate 
wavefronts in heterogeneous media by spheres ${ }^{1}$. This corresponds to a hyperbolic traveltime interpolation. For the constant velocity model the hyperbolic approximation is not only an approximation but equals the exact result.

To derive a hyperbolic traveltime approximation not $\tau$ is expanded into a Taylor series but its square, $\tau^{2}$. As for the parabolic approximation, the expansion is carried out up to order two

$$
\begin{aligned}
\tau^{2}\left(s_{i}, g_{i}\right)= & \tau_{0}^{2}+\left.\Delta s_{i} \frac{\partial \tau^{2}}{\partial s_{i}}\right|_{\hat{\boldsymbol{s}}_{0}, \hat{\boldsymbol{g}}_{0}}+\left.\Delta g_{i} \frac{\partial \tau^{2}}{\partial g_{i}}\right|_{\hat{\boldsymbol{s}}_{0}, \hat{\boldsymbol{g}}_{0}}+\left.\Delta s_{i} \Delta g_{j} \frac{\partial^{2} \tau^{2}}{\partial s_{i} \partial g_{j}}\right|_{\hat{\boldsymbol{s}}_{0}, \hat{\boldsymbol{g}}_{0}} \\
& +\left.\frac{1}{2} \Delta s_{i} \Delta s_{j} \frac{\partial^{2} \tau^{2}}{\partial s_{i} \partial s_{j}}\right|_{\hat{\boldsymbol{s}}_{0}, \hat{\boldsymbol{g}}_{0}}+\left.\frac{1}{2} \Delta g_{i} \Delta g_{j} \frac{\partial^{2} \tau^{2}}{\partial g_{i} \partial g_{j}}\right|_{\hat{\boldsymbol{s}}_{0}, \hat{\boldsymbol{g}}_{0}}+\mathcal{O}(3)
\end{aligned}
$$

Applying the chain rule, $[f(g(x))]^{\prime}=f^{\prime}(g) \cdot g^{\prime}(x)$, and inserting the abbreviations (3.1.2) and (3.1.3) into Equation (3.2.1), this yields

$$
\begin{aligned}
\tau^{2}(\hat{\boldsymbol{s}}, \hat{\boldsymbol{g}})= & \left(\tau_{0}-\hat{\boldsymbol{p}}_{0}^{\top} \Delta \hat{\boldsymbol{s}}+\hat{\boldsymbol{q}}_{0}^{\top} \Delta \hat{\boldsymbol{g}}\right)^{2}+\tau_{0}\left(-2 \Delta \hat{\boldsymbol{s}}^{\top} \underline{\hat{\mathbf{N}}} \Delta \hat{\boldsymbol{g}}\right. \\
& \left.-\Delta \hat{\boldsymbol{s}}^{\top} \underline{\hat{S}} \Delta \hat{\boldsymbol{s}}+\Delta \hat{\boldsymbol{g}}^{\top} \underline{\hat{\mathrm{G}}} \Delta \hat{\boldsymbol{g}}\right)+\mathcal{O}(3) .
\end{aligned}
$$

This equation is the hyperbolic traveltime expansion. As above for the parabolic form, the coefficients can be determined from traveltime tables. I give the same example as above, using the traveltimes $\tau_{0}, \tau_{1}$, and $\tau_{2}$ (cf. Figure 3.1) and inserting them into (3.2.2):

$$
\begin{array}{lrl}
\tau_{1}^{2} & =\tau_{0}^{2}+q_{x_{0}}^{2} \Delta g_{x}^{2}-2 \tau_{0} q_{x_{0}} \Delta g_{x}+\tau_{0} G_{x x} \Delta g_{x}^{2} \\
\tau_{2}^{2} & =\tau_{0}^{2}+q_{x_{0}}^{2} \Delta g_{x}^{2}+2 \tau_{0} q_{x_{0}} \Delta g_{x}+\tau_{0} G_{x x} \Delta g_{x}^{2} \\
\hline \text { Sum: } \quad \tau_{1}^{2}+\tau_{2}^{2} & =2 \tau_{0}^{2}+2 q_{x_{0}}^{2} \Delta g_{x}^{2} & +2 \tau_{0} G_{x x} \Delta g_{x}^{2} \\
\text { Difference: } & \tau_{1}^{2}-\tau_{2}^{2} & =
\end{array}
$$

Solving for $q_{x_{0}}$ and $G_{x x}$ yields

$$
q_{x_{0}}=\frac{\tau_{2}^{2}-\tau_{1}^{2}}{4 \tau_{0} \Delta g_{x}} \quad \text { and } \quad G_{x x}=\frac{\tau_{2}^{2}+\tau_{1}^{2}-2 \tau_{0}^{2}}{2 \tau_{0} \Delta g_{x}^{2}}-\frac{q_{x_{0}}^{2}}{\tau_{0}}
$$

The remaining coefficients can be determined accordingly. For the $z$ derivatives Equations (3.1.7) and (3.1.10) to (3.1.15) apply without changes in isotropic media. Expressions for the hyperbolic coefficients are given in Appendix D for isotropic and anisotropic media.

\footnotetext{
${ }^{1}$ This is a simplification. In $3-\mathrm{D}$, an ellipse would be more appropriate. However, the method approximates the local wavefront by a general surface of second order, meaning, that the two main radii of curvature can even have different sign.
} 


\subsection{Accuracy of the Coefficients}

I have tested the accuracy of the method on two analytic models: a homogeneous model, and a gradient model with $V=a+b z$. The velocity at the source (at depth $z_{s}$ ) is given by $V_{s}=a+b z_{s}$. The coefficients that were determined from the traveltime expansions were compared to analytic coefficients. Expressions for the analytic coefficients for both models are given in Appendix D.

The constant velocity medium has a velocity $V=3000 \mathrm{~m} / \mathrm{s}$, and the gradient model has $a=3000 \mathrm{~m} / \mathrm{s}$ and $b=0.5 \mathrm{~s}^{-1}$. Traveltimes for both models were given on a $100 \mathrm{~m}$ grid, i.e. a grid with $100 \mathrm{~m}$ spacing. To test the accuracy of the method itself analytic traveltimes have been used. Since an analytic solution is not generally available, a second test was carried out with traveltimes computed with a finite differences eikonal solver (FDES) using the Vidale (1990) algorithm. These traveltimes were computed on a 10m fine grid and resampled to the $100 \mathrm{~m}$ grid. This was necessary because FDES need fine grids to provide sufficient accuracy. For both models and both sets of traveltime tables the coefficients were determined twice: from the hyperbolic and the parabolic variant as shown above. The results were compared to the analytic coefficients. For the gradient model the analytic coefficients are shown in Figure 3.2.

Table 3.1 gives the median relative errors for the coefficients of the constant velocity model, in Table 3.2 the same is found for the gradient model. I use median errors rather than average errors because they are less sensitive to outliers. The errors given are relative errors, meaning

$$
\Delta f_{\mathrm{rel}}=\frac{f-f_{0}}{f_{0}},
$$

where $\Delta f_{\text {rel }}$ is the relative error of the quantity $f$ compared with the reference value $f_{0}$. There are regions with small reference value (e.g., if $f$ is a traveltime near the source) where the relative errors appear higher due to the division by the small $f_{0}$. This should be kept in mind when dealing with relative errors.

Tables 3.1 and 3.2 show that the hyperbolic results are superior to the parabolic ones. This confirms that any wavefront can locally be well approximated by a sphere, or, more generally, a surface of second order. The coefficients from the numerically computed input traveltimes are less accurate than for analytic traveltimes. Figure 3.3 demonstrates the spatial distribution of the errors for both hyperbolic and parabolic coefficients from analytic traveltimes and hyperbolic coefficients from analytic traveltimes for the gradient model. Figure 3.4 shows relative errors of the hyperbolic coefficients from Vidale traveltimes, also for the gradient model.

Figure 3.3 also states that the hyperbolic form yields smaller errors than the parabolic one. This is most obvious in the near source region but not restricted to it. The same holds for the coefficients from Vidale traveltimes: Figure 3.4 shows the errors of the hyperbolic coefficients from Vidale traveltimes. In all plots, there exist regions with higher errors, as, for example, the stripe in the front of $G_{z z}$ or the top surface of $q_{z}$ (see Figures 3.3 and 3.4). Comparing these errors with the values of $G_{z z}$ and $q_{z}$ in 

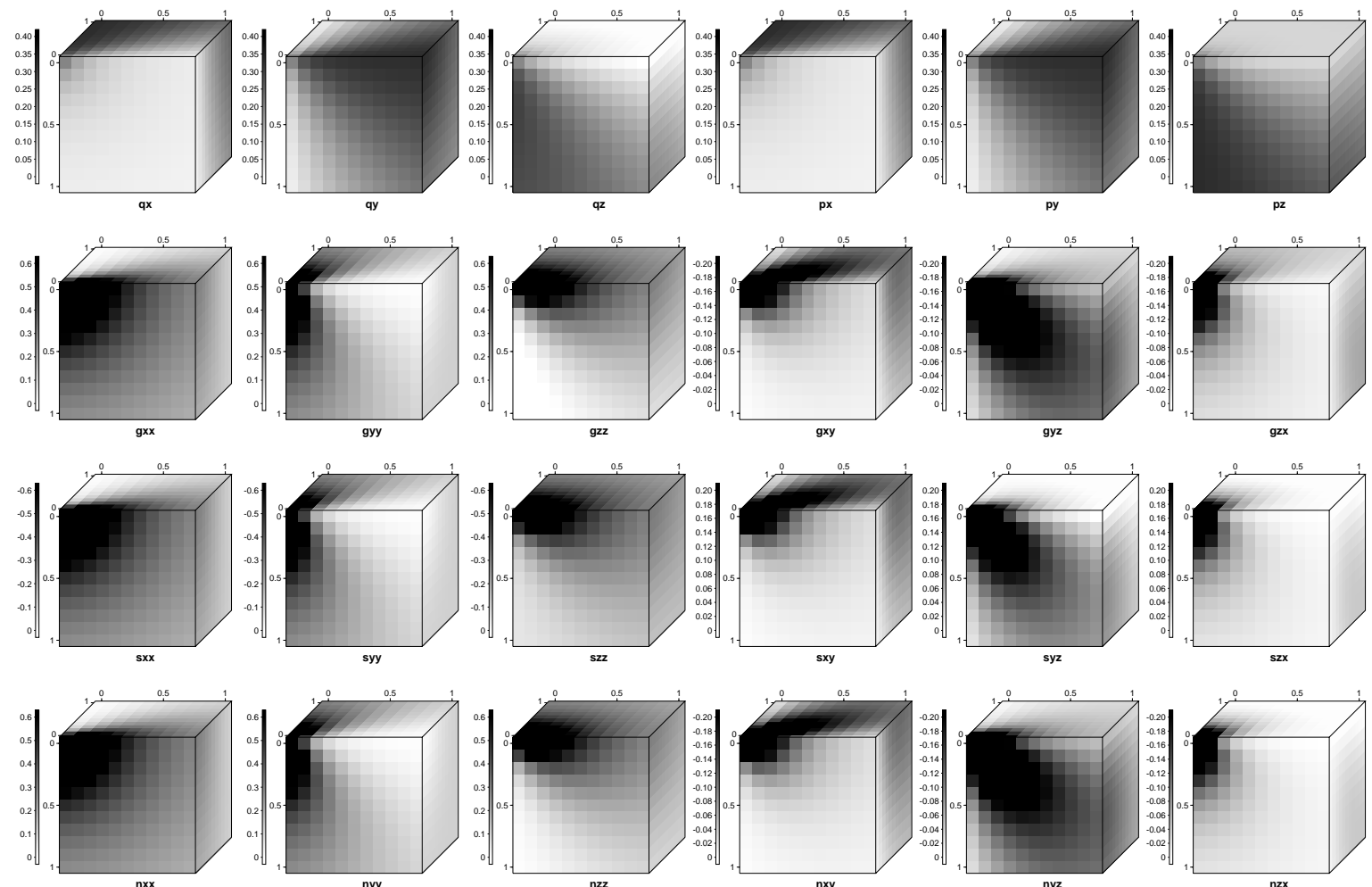

Gradient model, analytic coefficients
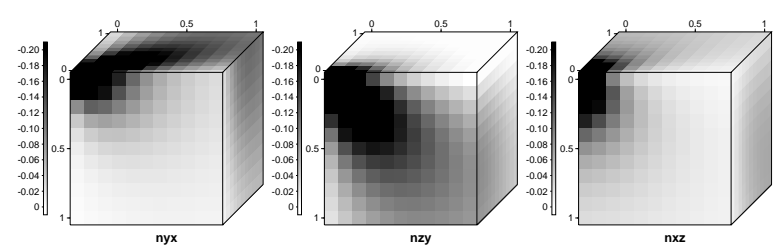

Figure 3.2: Analytic coefficients for a gradient model: the slownesses in [s/km] are given in the top row, followed by the second order derivatives in $\left[\mathrm{s} / \mathrm{km}^{2}\right]$. Absolute values are given. Axis labels are omitted to save space. The $z$ axis corresponds to the vertical direction (depth), the $y$ axis to the horizontal direction. The $x$ axis is perpendicular to the page. Distances are given in $[\mathrm{km}]$. The source is located at $-100 \mathrm{~m}$ in either direction.

Figure 3.2 explains them with the exaggerated impact of very small values of $G_{z z}$ and $q_{z}$ (see the discussion on relative errors above). Figure 3.4 shows that the coefficients from Vidale traveltimes suffer from more errors than those from analytic traveltimes. For nearly all coefficients a region of higher error is visibly present which follows a $45^{\circ}$ line starting from the source position. This region coincides with errors of the input traveltimes from the Vidale algorithm given in Figure 3.5. The error distribution of the parabolic coefficients from Vidale traveltime corresponds to that of the parabolic coefficients from analytic traveltimes with additional contributions due to the errors in the Vidale traveltimes. Since no new insights can be gained from it, the parabolic error distribution of coefficients from Vidale traveltimes is not displayed in a Figure.

Error plots for the homogeneous model are not given here. The spatial distribution of 
Table 3.1: Median of relative errors in percent of the coefficients for the constant velocity model. Hyperbolic coefficient errors from analytic traveltimes reflect the numerical noise of 32 bit words.

\begin{tabular}{|l|l|l|l|l|}
\hline & \multicolumn{2}{|c|}{ Analytic traveltimes } & \multicolumn{2}{|c|}{ Vidale traveltimes } \\
\hline & $\begin{array}{c}\text { hyperbolic } \\
10^{-3}\end{array}$ & parabolic & hyperbolic & parabolic \\
\hline$q_{x}$ & 0.040 & 0.245 & 0.229 & 0.436 \\
$q_{y}$ & 0.040 & 0.245 & 0.229 & 0.436 \\
$q_{z}$ & 0.040 & 0.245 & 0.136 & 0.264 \\
\hline$p_{x}$ & 0.040 & 0.245 & 0.229 & 0.436 \\
$p_{y}$ & 0.040 & 0.245 & 0.229 & 0.436 \\
$p_{z}$ & 0.055 & 0.451 & 0.147 & 0.406 \\
\hline$G_{x x}$ & 1.190 & 0.162 & 1.63 & 1.73 \\
$G_{y y}$ & 1.190 & 0.162 & 1.63 & 1.73 \\
$G_{z z}$ & 1.210 & 0.162 & 1.62 & 1.57 \\
\hline$G_{x y}$ & 0.725 & 0.886 & 1.17 & 2.13 \\
$G_{y z}$ & 0.720 & 0.886 & 1.40 & 1.96 \\
$G_{z x}$ & 0.720 & 0.886 & 1.40 & 1.96 \\
\hline$S_{x x}$ & 1.190 & 0.162 & 1.63 & 1.73 \\
$S_{y y}$ & 1.190 & 0.162 & 1.63 & 1.73 \\
$S_{z z}$ & 2.860 & 0.361 & 1.86 & 2.00 \\
\hline$S_{x y}$ & 0.725 & 0.886 & 1.17 & 2.13 \\
$S_{y z}$ & 3.16 & 0.455 & 2.05 & 2.10 \\
$S_{z x}$ & 3.16 & 0.455 & 2.05 & 2.10 \\
\hline$N_{x x}$ & 0.291 & 0.637 & 0.931 & 1.61 \\
$N_{y y}$ & 0.291 & 0.637 & 0.931 & 1.61 \\
$N_{z z}$ & 0.509 & 1.48 & 0.976 & 1.65 \\
\hline$N_{x y}$ & 0.725 & 0.886 & 1.17 & 2.13 \\
$N_{y z}$ & 0.720 & 0.886 & 1.40 & 1.96 \\
$N_{z x}$ & 1.14 & 1.33 & 1.57 & 2.66 \\
\hline$N_{y x}$ & 0.725 & 0.886 & 1.17 & 2.13 \\
$N_{z y}$ & 1.14 & 1.33 & 1.57 & 2.66 \\
$N_{x z}$ & 0.720 & 0.886 & 1.40 & 1.96 \\
\hline & & & & \\
\hline & & & \\
\hline
\end{tabular}

the errors looks similar to the gradient model. The hyperbolic variant has again much smaller errors than the parabolic one. It would be, however, misleading to base the superiority of the hyperbolic variant only on the homogeneous example, because in this case the hyperbolic traveltime expansion up to second order yields the exact traveltime (see Appendix C). Therefore, the accuracy of the hyperbolic coefficients from analytic traveltimes is expected to be within machine precision, which is confirmed by Table 3.1. Similarly, the accuracy of the hyperbolic coefficients from Vidale traveltimes is dominated by errors in the input traveltimes. 
Table 3.2: Median of relative errors in percent of the coefficients for the gradient model.

\begin{tabular}{|l|l|l|l|l|}
\hline & \multicolumn{2}{|c|}{ Analytic traveltimes } & \multicolumn{2}{c|}{ Vidale traveltimes } \\
\hline & $\begin{array}{c}\text { hyperbolic } \\
\cdot 10^{-3}\end{array}$ & parabolic & hyperbolic & parabolic \\
\hline$q_{x}$ & 4.17 & 0.248 & 0.206 & 0.41 \\
$q_{y}$ & 4.17 & 0.248 & 0.206 & 0.41 \\
$q_{z}$ & 131.0 & 0.367 & 0.139 & 0.305 \\
\hline$p_{x}$ & 4.17 & 0.248 & 0.206 & 0.41 \\
$p_{y}$ & 4.17 & 0.248 & 0.206 & 0.41 \\
$p_{z}$ & 6.73 & 0.316 & 0.098 & 0.306 \\
\hline$G_{x x}$ & 2.07 & 0.165 & 1.21 & 1.32 \\
$G_{y y}$ & 2.07 & 0.165 & 1.21 & 1.32 \\
$G_{z z}$ & 111.0 & 0.204 & 1.46 & 1.48 \\
\hline$G_{x y}$ & 8.64 & 0.884 & 0.913 & 1.79 \\
$G_{y z}$ & 94.9 & 0.767 & 1.05 & 1.40 \\
$G_{z x}$ & 94.9 & 0.767 & 1.05 & 1.40 \\
\hline$S_{x x}$ & 2.07 & 0.165 & 1.21 & 1.32 \\
$S_{y y}$ & 2.07 & 0.165 & 1.21 & 1.32 \\
$S_{z z}$ & 7.65 & 0.209 & 0.664 & 0.688 \\
\hline$S_{x y}$ & 8.64 & 0.884 & 0.913 & 1.79 \\
$S_{y z}$ & 5.56 & 0.578 & 1.46 & 1.60 \\
$S_{z x}$ & 5.56 & 0.578 & 1.46 & 1.60 \\
\hline$N_{x x}$ & 8.16 & 0.643 & 0.772 & 1.48 \\
$N_{y y}$ & 8.16 & 0.643 & 0.772 & 1.48 \\
$N_{z z}$ & 106.0 & 1.22 & 0.913 & 1.34 \\
\hline$N_{x y}$ & 8.64 & 0.884 & 0.913 & 1.79 \\
$N_{y z}$ & 94.9 & 0.767 & 1.05 & 1.40 \\
$N_{z x}$ & 18.6 & 1.45 & 1.37 & 2.55 \\
\hline$N_{y x}$ & 8.64 & 0.884 & 0.913 & 1.79 \\
$N_{z y}$ & 18.6 & 1.45 & 1.37 & 2.55 \\
$N_{x z}$ & 94.9 & 0.767 & 1.05 & 1.40 \\
\hline & & & & \\
\hline
\end{tabular}



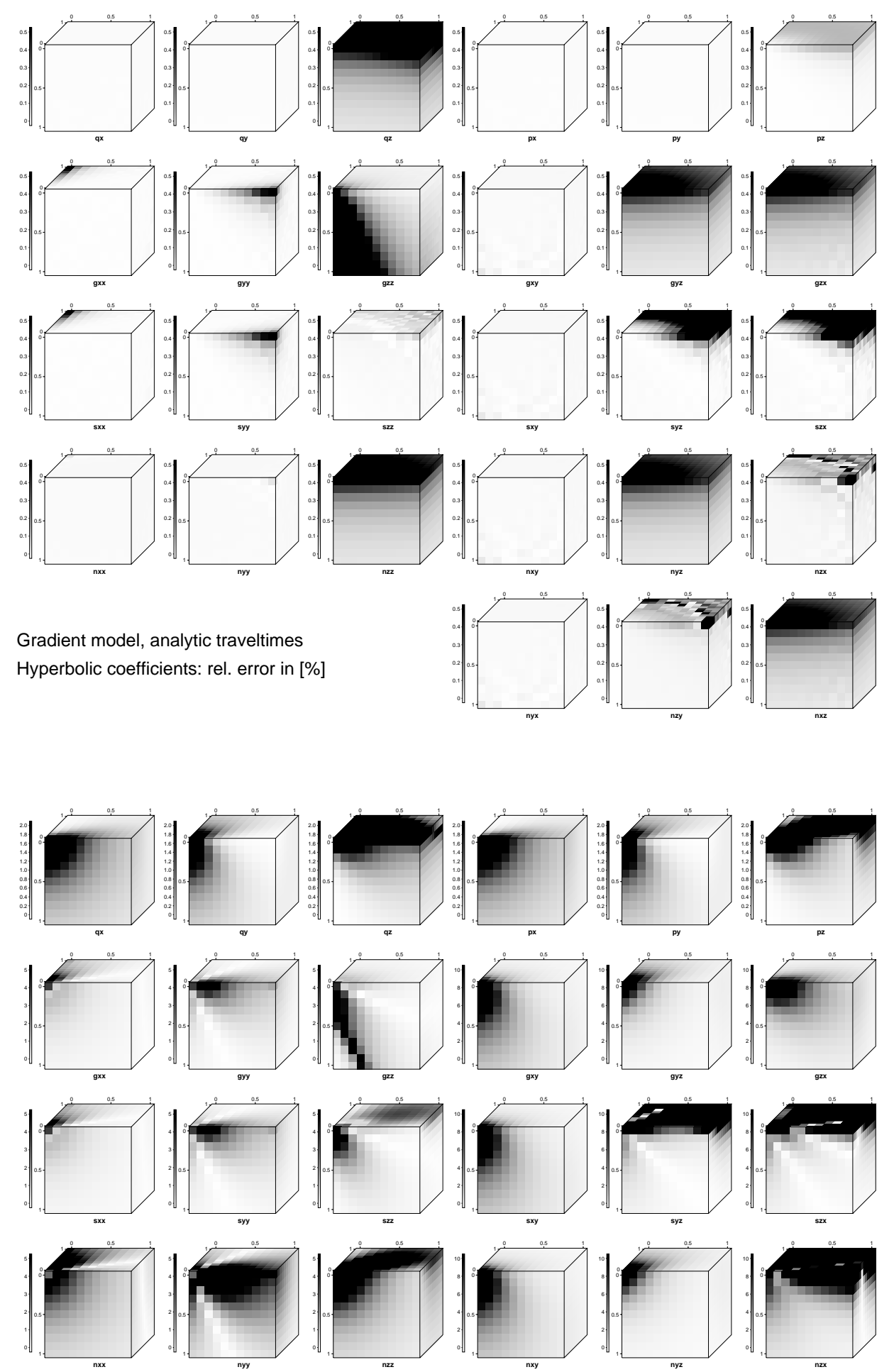

Gradient model, analytic traveltimes Parabolic coefficients: rel. error in [\%]
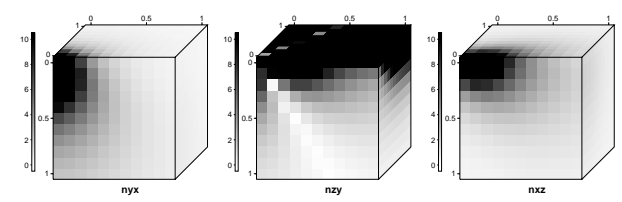

Figure 3.3: Relative errors of coefficients from analytic traveltimes for the gradient model. On top for the hyperbolic, bottom for parabolic coefficients. Note the different error scales. The source is at $-100 \mathrm{~m}$ in either direction. 


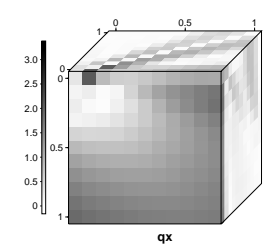

qx
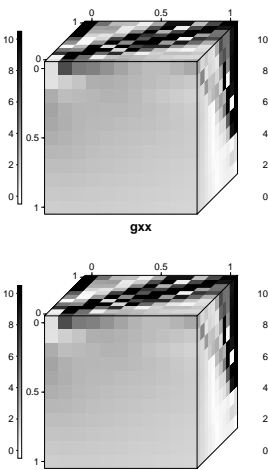

$\mathbf{s x x}$

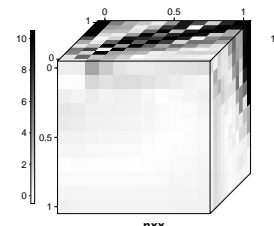

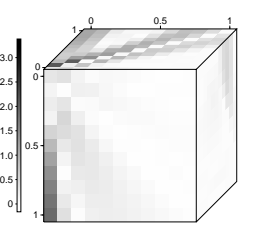
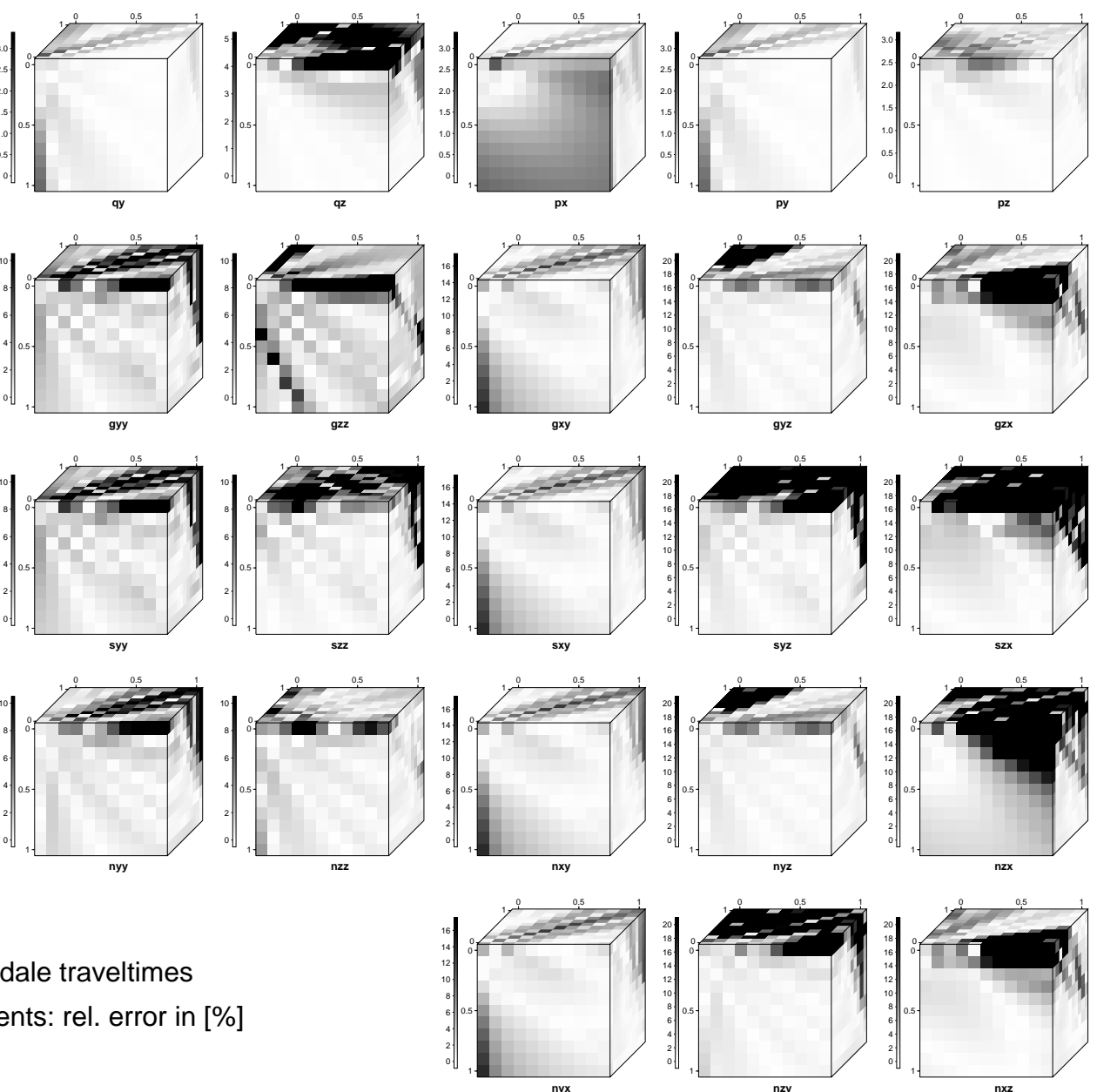

Gradient model, Vidale traveltimes Hyperbolic coefficients: rel. error in [\%]

Figure 3.4: Relative errors of coefficients from Vidale traveltimes for the gradient model. In addition to regions with higher relative errors caused by small values of the coefficients under consideration, other regions with higher errors follow the errors of the Vidale input traveltimes, see Figure 3.5. 


$$
\text { A }
$$




\subsection{Examples}

The parabolic and hyperbolic expressions for traveltimes can be used for traveltime interpolation with high accuracy, once the according coefficient sets are known. This includes interpolation not only in between receivers but also in between sources. For each of the following examples both parabolic and hyperbolic coefficients were determined using the procedure described in Sections 3.1 and 3.2. Subsequent interpolation with the parabolic formula (3.1.4) respectively the hyperbolic variant (3.2.2) was applied.

\section{Constant Velocity Model}

The first example is a model with a constant velocity of $3000 \mathrm{~m} / \mathrm{s}$. I have used analytic traveltimes and traveltimes from an FDES using the Vidale algorithm (see Section 3.3) as input data to validate the accuracy. Errors from analytic input traveltimes are only due to the method itself and possibly roundoff errors whereas errors from Vidale traveltimes give an estimate of the accuracy under more realistic conditions. The example model is a cube of $101 \times 101 \times 101$ grid points with $10 \mathrm{~m}$ grid spacing. The source is centred in the top surface. Input traveltimes were given on a cubical $100 \mathrm{~m}$ coarse grid. The distances in source position were also $100 \mathrm{~m}$ in either direction. The interpolations onto a $10 \mathrm{~m}$ fine grid were each carried out twice: for the original source position and for a source shifted by $50 \mathrm{~m}$ in $x$ and $y$. The results are compared to analytic and Vidale reference traveltimes, respectively, on a fine $10 \mathrm{~m}$ grid. The resulting relative traveltime errors are displayed in Figure 3.6 for the analytic input traveltimes and in Figure 3.7 for the Vidale input traveltimes. The results look similar for analytic and Vidale traveltimes: hyperbolic coefficients yield higher accuracy than parabolic ones. The magnitude of the errors from Vidale traveltime is the same as those from analytic input traveltimes for the parabolic variant. For the hyperbolic variant analytic traveltimes lead to exact coefficients, therefore the errors from the interpolation are within machine precision. The results from hyperbolic interpolation of Vidale input traveltimes give a more realistic estimate. The pattern in the error distribution can be explained as follows: traveltimes were interpolated onto the fine grid using the coefficients of the nearest coarse grid point. This leads to the discontinuities in the error plots where the area of fine grid points surrounding the coarse grid point under consideration ends. The traveltime errors are summarised in Table 3.3 together with results for a trilinear interpolation (without source shift) using the $100 \mathrm{~m}$ coarse grid traveltimes as input data. A layer of $100 \mathrm{~m}$ thickness under the source was excluded from the statistics since the errors near the source are dominated by small traveltimes (see the discussion of relative errors in Section 3.3). The hyperbolic interpolation is superior to the parabolic variant. Both exceed the trilinear interpolation by far. Note that median errors are used rather than mean errors. This is due to the stability of the median concerning outliers. Therefore, the median error is a more reliable value compared to the mean error. 

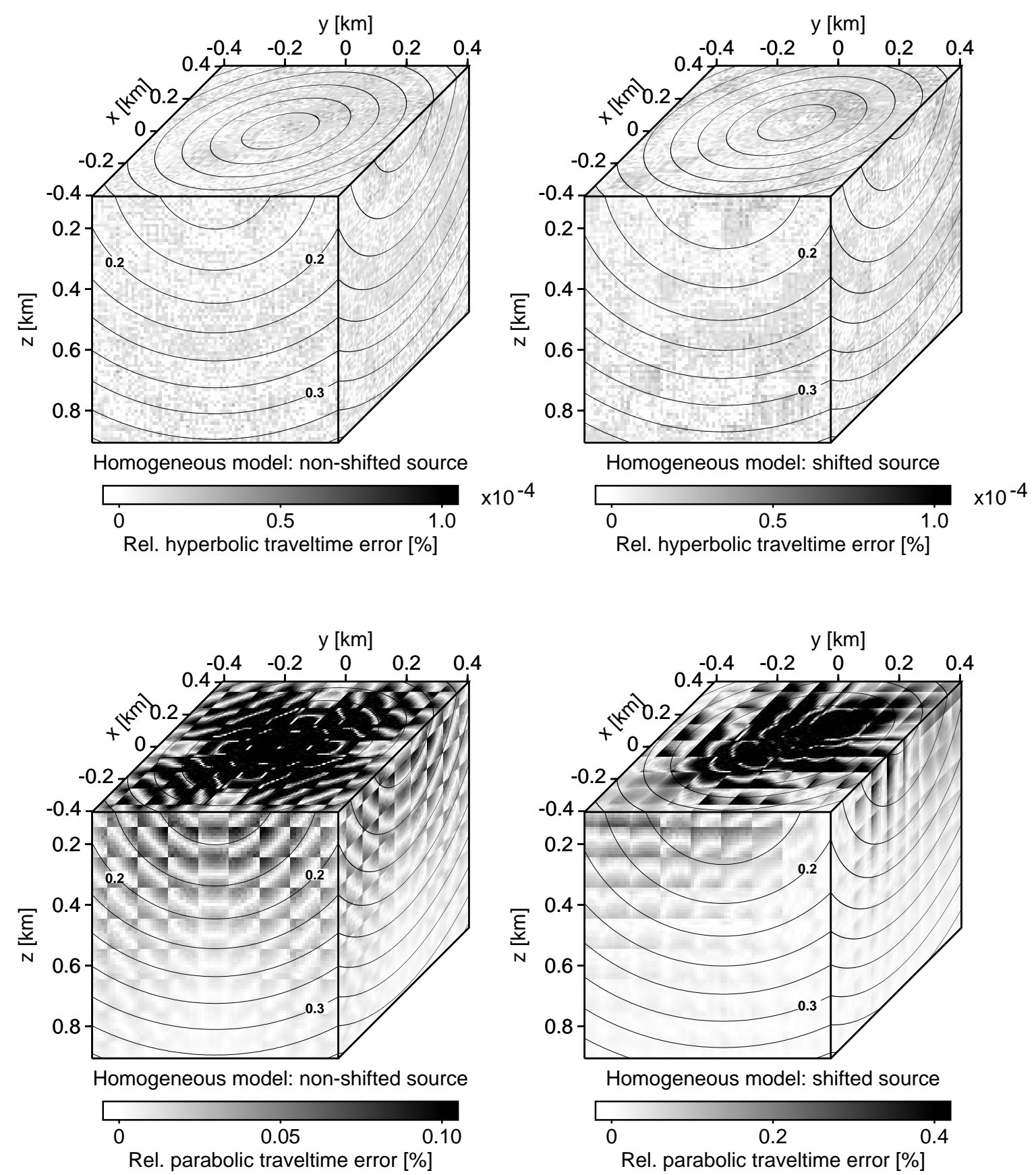

Figure 3.6: Relative traveltime errors for a homogeneous velocity model using analytic input traveltimes. Top: errors using the hyperbolic interpolation if only receivers are interpolated (left) and for both source and receiver interpolation (right). Isochrones are given in seconds. Bottom: the same as above but using the parabolic variant. The relative errors near the source appear exaggerated because there the traveltimes are very small. Note the different error scales. 


\section{Traveltime Interpolation for a Homogeneous Model using Vidale Input Traveltimes}
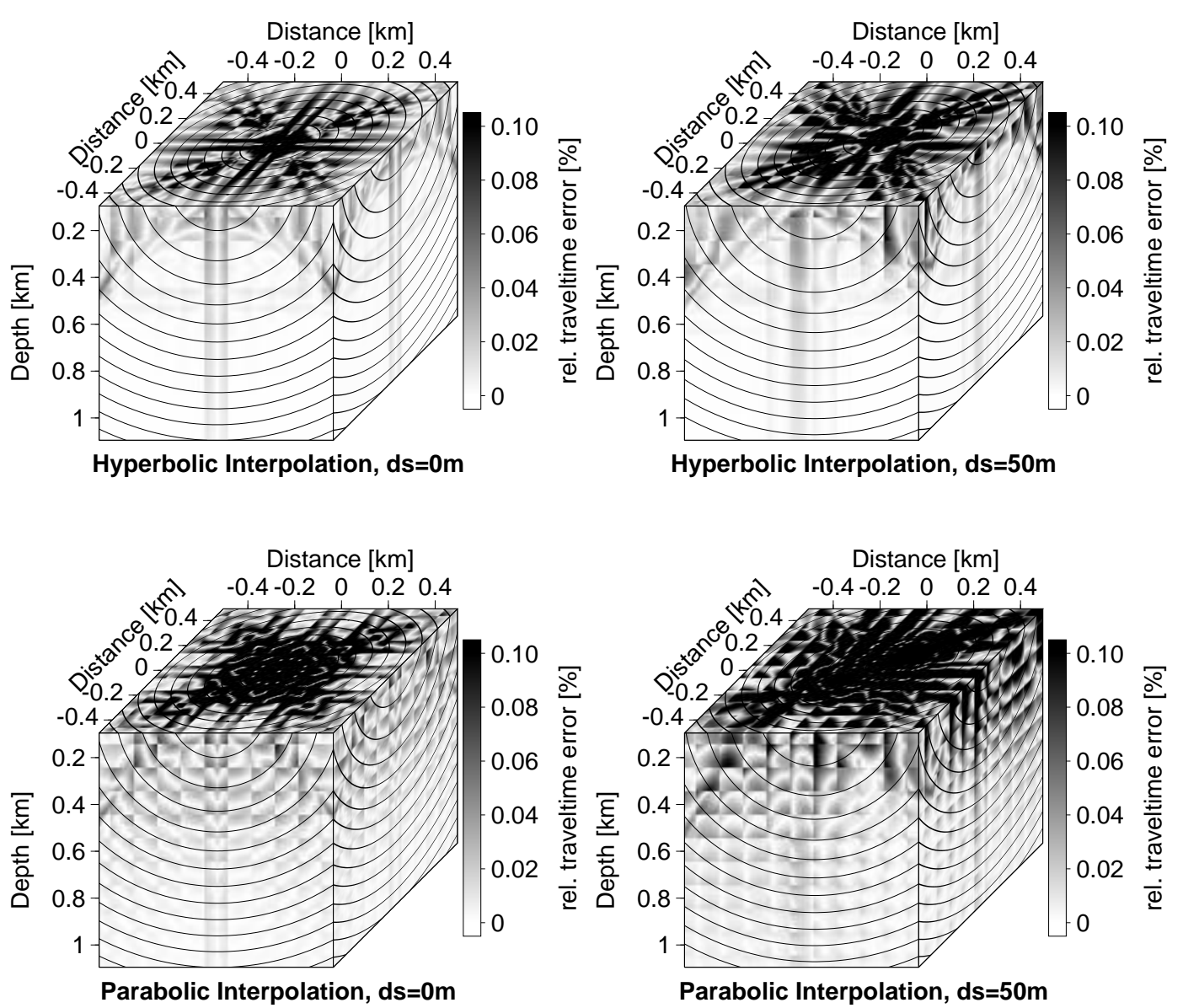

Figure 3.7: Relative traveltime errors for a homogeneous velocity model using Vidale input traveltimes. Top: errors using the hyperbolic interpolation if only receivers are interpolated (left) and for both source and receiver interpolation (right). Isochrones are given in seconds. Bottom: the same as above but using the parabolic variant. The relative errors near the source appear exaggerated because there the traveltimes are very small. 
Table 3.3: Errors of traveltime interpolation for the homogeneous velocity model excluding a layer of $100 \mathrm{~m}$ thickness under the source.

\begin{tabular}{|c|c|c|c|c|c|}
\hline Interpolation & $\begin{array}{r}\text { Source shift } \\
\Delta s_{x}=\Delta s_{y}\end{array}$ & $\begin{array}{l}\text { Median of } \\
\text { rel. error }\end{array}$ & $\begin{array}{l}\text { Maximum } \\
\text { rel. error }\end{array}$ & $\begin{array}{l}\text { Median of } \\
\text { abs. error }\end{array}$ & $\begin{array}{l}\text { Maximum } \\
\text { abs. error }\end{array}$ \\
\hline \multicolumn{6}{|c|}{ Analytic input traveltimes } \\
\hline hyperbolic & $0 \mathrm{~m}$ & $<10^{-6} \%$ & $2.2 \cdot 10^{-5} \%$ & $<10^{-6} \mathrm{~ms}$ & $5.96 \cdot 10^{-5} \mathrm{~ms}$ \\
\hline hyperbolic & $50 \mathrm{~m}$ & $<10^{-6} \%$ & $3.35 \cdot 10^{-5} \%$ & $<10^{-6} \mathrm{~ms}$ & $8.94 \cdot 10^{-5} \mathrm{~ms}$ \\
\hline parabolic & $0 \mathrm{~m}$ & $0.0088 \%$ & $4.90 \%$ & $0.023 \mathrm{~ms}$ & $2.00 \mathrm{~ms}$ \\
\hline parabolic & $50 \mathrm{~m}$ & $0.0137 \%$ & $8.38 \%$ & $0.035 \mathrm{~ms}$ & $4.44 \mathrm{~ms}$ \\
\hline trilinear & $0 \mathrm{~m}$ & $0.4080 \%$ & $36.3 \%$ & $0.878 \mathrm{~ms}$ & $8.54 \mathrm{~ms}$ \\
\hline \multicolumn{6}{|c|}{ Vidale input traveltimes } \\
\hline hyperbolic & $0 \mathrm{~m}$ & $0.0017 \%$ & $0.82 \%$ & $0.0038 \mathrm{~ms}$ & $0.446 \mathrm{~ms}$ \\
\hline hyperbolic & $50 \mathrm{~m}$ & $0.0048 \%$ & $2.18 \%$ & $0.0118 \mathrm{~ms}$ & $0.952 \mathrm{~ms}$ \\
\hline parabolic & $0 \mathrm{~m}$ & $0.0102 \%$ & $4.37 \%$ & $0.0257 \mathrm{~ms}$ & $1.83 \mathrm{~ms}$ \\
\hline parabolic & $50 \mathrm{~m}$ & $0.0150 \%$ & $8.39 \%$ & $0.0381 \mathrm{~ms}$ & $4.47 \mathrm{~ms}$ \\
\hline \multicolumn{6}{|c|}{ Rel. errors of the Vidale input traveltimes } \\
\hline & & $0.0638 \%$ & $0.824 \%$ & $0.17 \mathrm{~ms}$ & $0.752 \mathrm{~ms}$ \\
\hline
\end{tabular}

\section{Constant Velocity Gradient Model}

The second model is again an example where the analytic solution is known. It consists of $121 \times 121 \times 121$ grid points with $10 \mathrm{~m}$ grid spacing and has a constant velocity gradient of $\partial V / \partial z=0.5 \mathrm{~s}^{-1}$. The velocity at the source is $3000 \mathrm{~m} / \mathrm{s}$. The source is positioned in the centre of the top surface. As before, a coarse grid of $100 \mathrm{~m}$ was used for the analytic and Vidale input traveltimes. The results are shown in Figure 3.8 and Figure 3.8, and in Table 3.4, where a layer of $100 \mathrm{~m}$ depth under the source was excluded from the statistics. Again the hyperbolic results are more accurate than the parabolic ones and both second order interpolations are far superior to trilinear interpolation. Table 3.5 gives result for a model with the same geometry and gradient as the previous one, but with a velocity of $1500 \mathrm{~m} / \mathrm{s}$ at the source (given for analytic traveltimes only). For both gradient models, the errors of the hyperbolic variant using analytic input traveltimes are smaller for the shifted source case. This is correct - although it seems counter-intuitive at first sight. The reason is that the errors caused by the change in receiver position have different sign than those caused by the source interpolation. Therefore in this special case the errors from source and receiver interpolation compensate each other.

The difference in quality between hyperbolic and parabolic interpolation for the gradient models is less than for the constant velocity model. This is due to the fact that for a homogeneous medium the hyperbolic approximation is equal to the analytic result (see the proof in Appendix C). The reason for the much higher accuracy of the parabolic and hyperbolic interpolation compared to trilinear interpolation is that the latter neglects the wavefront curvature. Unlike in the previous examples this is not only a problem in the 

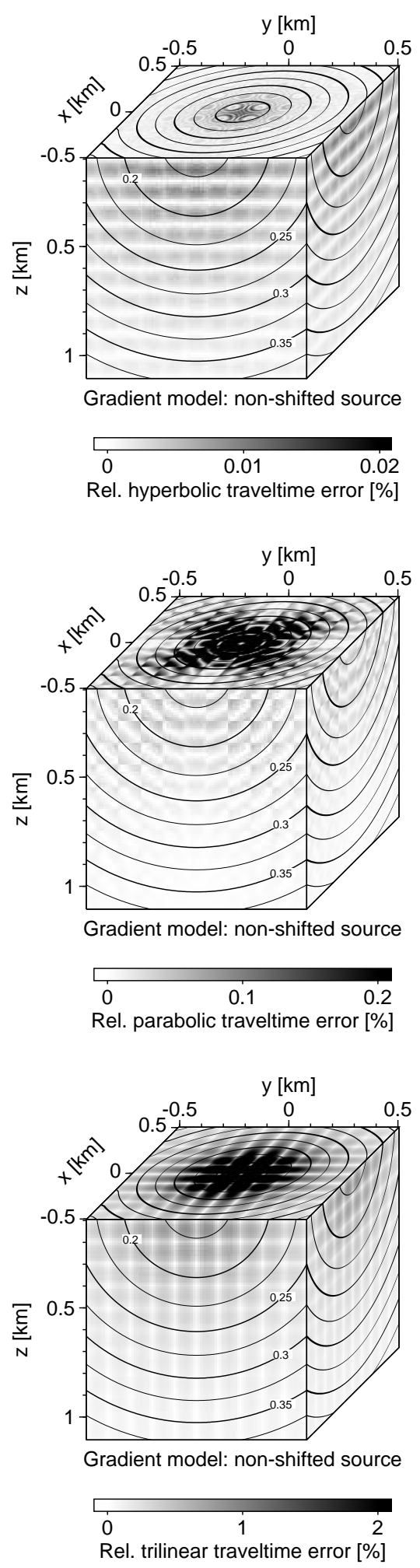
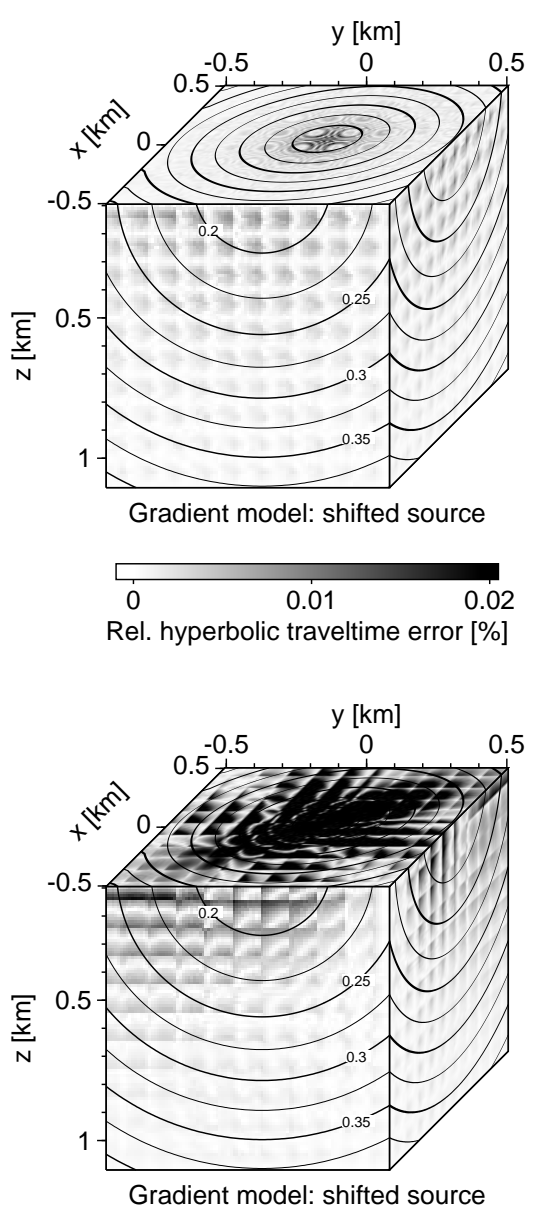

Gradient model: shifted source

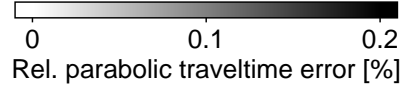

Figure 3.8: Relative traveltime errors for a constant velocity gradient model using analytic input traveltimes. Top: errors using the hyperbolic interpolation for the original source position (left) and for a shifted source (right). Middle: the same as above using parabolic interpolation. Bottom: as before but with trilinear interpolation. Note the different error scales on the plots. 


\section{Traveltime Interpolation for a Gradient Model using Vidale Input Traveltimes}
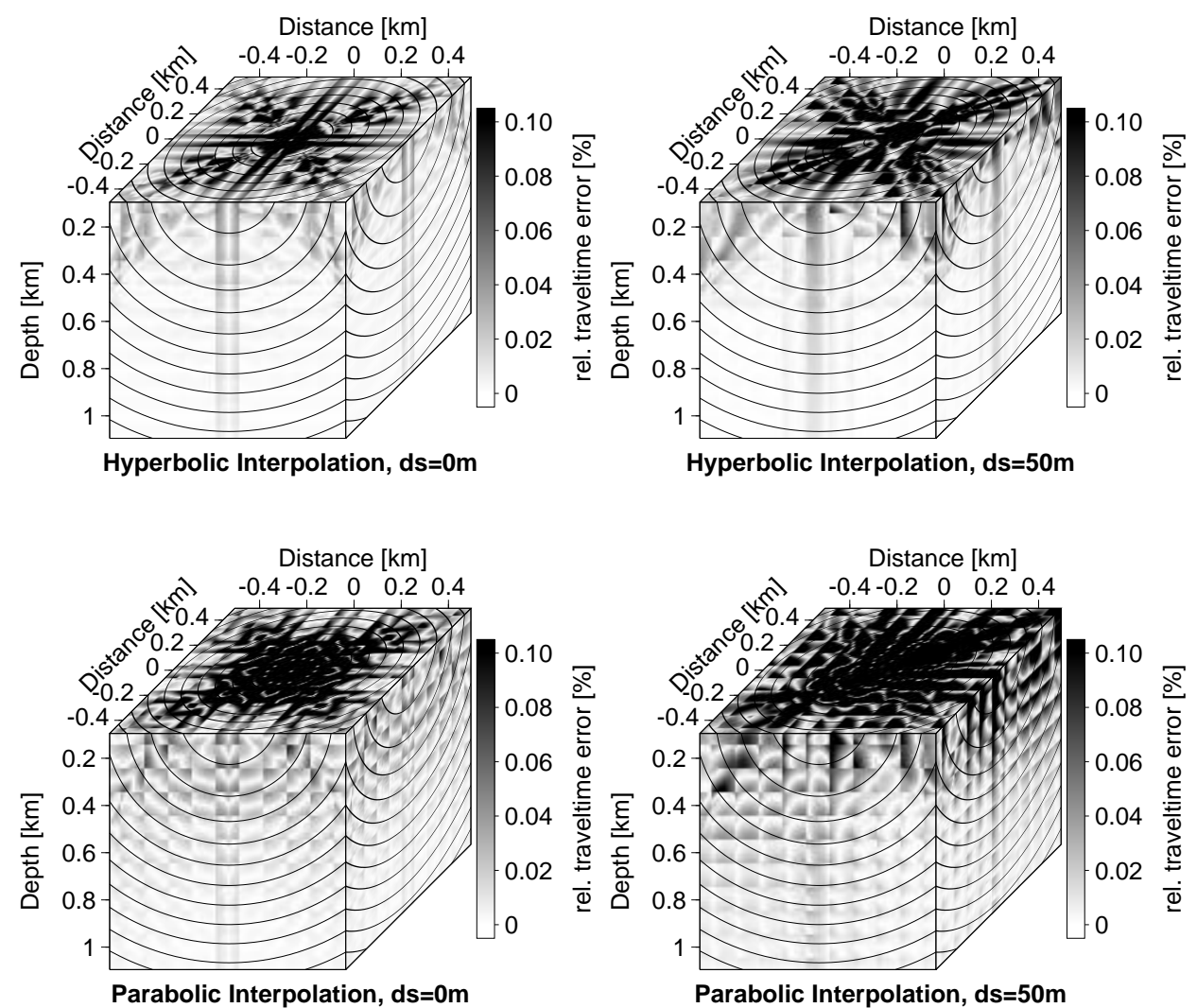

Figure 3.9: Relative traveltime errors for a constant velocity gradient model using Vidale input traveltimes. Top: errors using the hyperbolic interpolation for the original source position (left) and for a shifted source (right). Bottom: the same as above using parabolic interpolation. 
Table 3.4: Errors for the constant velocity gradient model $\left(V_{s}=3000 \mathrm{~m} / \mathrm{s}\right)$ with $100 \mathrm{~m}$ coarse grid spacing excluding a layer of $100 \mathrm{~m}$ depth under the source.

\begin{tabular}{|c|c|c|c|c|c|}
\hline Interpolation & $\begin{array}{r}\text { Source shift } \\
\Delta s_{x}=\Delta s_{y} \\
\end{array}$ & $\begin{array}{l}\text { Median of } \\
\text { rel. error }\end{array}$ & $\begin{array}{l}\text { Maximum } \\
\text { rel. error }\end{array}$ & $\begin{array}{l}\text { Median of } \\
\text { abs. error }\end{array}$ & $\begin{array}{l}\text { Maximum } \\
\text { abs. error }\end{array}$ \\
\hline \multicolumn{6}{|c|}{ Analytic input traveltimes } \\
\hline hyperbolic & $0 \mathrm{~m}$ & $0.002 \%$ & $0.137 \%$ & $0.004 \mathrm{~ms}$ & $0.064 \mathrm{~ms}$ \\
\hline hyperbolic & $50 \mathrm{~m}$ & $0.001 \%$ & $0.320 \%$ & $0.002 \mathrm{~ms}$ & $0.148 \mathrm{~ms}$ \\
\hline parabolic & $0 \mathrm{~m}$ & $0.009 \%$ & $4.9 \%$ & $0.021 \mathrm{~ms}$ & $1.99 \mathrm{~ms}$ \\
\hline parabolic & $50 \mathrm{~m}$ & $0.015 \%$ & $8.38 \%$ & $0.036 \mathrm{~ms}$ & $4.47 \mathrm{~ms}$ \\
\hline trilinear & $0 \mathrm{~m}$ & $0.282 \%$ & $14.5 \%$ & $0.677 \mathrm{~ms}$ & $5.52 \mathrm{~ms}$ \\
\hline \multicolumn{6}{|c|}{ Vidale input traveltimes } \\
\hline hyperbolic & $0 \mathrm{~m}$ & $0.003 \%$ & $0.822 \%$ & $0.0065 \mathrm{~ms}$ & $0.479 \mathrm{~ms}$ \\
\hline hyperbolic & $50 \mathrm{~m}$ & $0.005 \%$ & $2.1 \%$ & $0.0110 \mathrm{~ms}$ & $0.905 \mathrm{~ms}$ \\
\hline parabolic & $0 \mathrm{~m}$ & $0.010 \%$ & $4.36 \%$ & $0.0248 \mathrm{~ms}$ & $1.83 \mathrm{~ms}$ \\
\hline parabolic & $50 \mathrm{~m}$ & $0.016 \%$ & $8.4 \%$ & $0.0375 \mathrm{~ms}$ & $4.50 \mathrm{~ms}$ \\
\hline \multicolumn{6}{|c|}{ Rel. errors of the Vidale input traveltimes } \\
\hline & & $0.0719 \%$ & $0.829 \%$ & $0.181 \mathrm{~ms}$ & $0.745 \mathrm{~ms}$ \\
\hline
\end{tabular}

Table 3.5: Errors for the constant velocity gradient model $\left(V_{s}=1500 \mathrm{~m} / \mathrm{s}\right)$ with $100 \mathrm{~m}$ coarse grid spacing excluding a layer of $100 \mathrm{~m}$ depth under the source.

\begin{tabular}{|lr|rrrr|}
\hline Interpolation & $\begin{array}{r}\text { Source shift } \\
\Delta s_{x}=\Delta s_{y}\end{array}$ & $\begin{array}{r}\text { Median of } \\
\text { rel. error }\end{array}$ & $\begin{array}{r}\text { Maximum } \\
\text { rel. error }\end{array}$ & $\begin{array}{r}\text { Median of } \\
\text { abs. error }\end{array}$ & $\begin{array}{r}\text { Maximum } \\
\text { abs. error }\end{array}$ \\
\hline hyperbolic & $0 \mathrm{~m}$ & $0.003 \%$ & $0.263 \%$ & $0.012 \mathrm{~ms}$ & $0.240 \mathrm{~ms}$ \\
hyperbolic & $50 \mathrm{~m}$ & $0.002 \%$ & $0.616 \%$ & $0.008 \mathrm{~ms}$ & $0.566 \mathrm{~ms}$ \\
parabolic & $0 \mathrm{~m}$ & $0.009 \%$ & $4.91 \%$ & $0.042 \mathrm{~ms}$ & $3.94 \mathrm{~ms}$ \\
parabolic & $50 \mathrm{~m}$ & $0.015 \%$ & $8.39 \%$ & $0.071 \mathrm{~ms}$ & $9.0 \mathrm{~ms}$ \\
\hline
\end{tabular}

near-source region but anywhere where locally higher wavefront curvatures occur. It is especially very common for more complex velocity models.

\section{Marmousi Model}

As an example for a complex velocity model I have employed a 3-D extension of the Marmousi model (Versteeg and Grau, 1991), shown in Figure 3.10. The model was 100-fold smoothed using a three point operator with a central weight of 0.5 (cf., e.g., Ettrich and Gajewski 1996). This corresponds to removing all wavelengths below $667 \mathrm{~m}$ from the model. Smoothing of velocities is required if traveltime generators based on the ray method are used. Traveltimes were computed with a 3-D-FD eikonal solver using the Vidale (1990) algorithm on a $12.5 \mathrm{~m}$ fine grid. These were used as reference data as well as for the input traveltime tables which were obtained from the fine grid data by 
resampling them onto a $125 \mathrm{~m}$ coarse grid. The resulting interpolated traveltimes were compared to the reference data on the fine grid. The relative traveltime errors for the hyperbolic interpolation (no source shift) are shown in Figure 3.11. Figure 3.12 shows the absolute traveltime errors using the hyperbolic interpolation (again, the source was not shifted). Table 3.6 summarises the errors. A layer of $62.5 \mathrm{~m}$ depth under the source was excluded from the statistics.

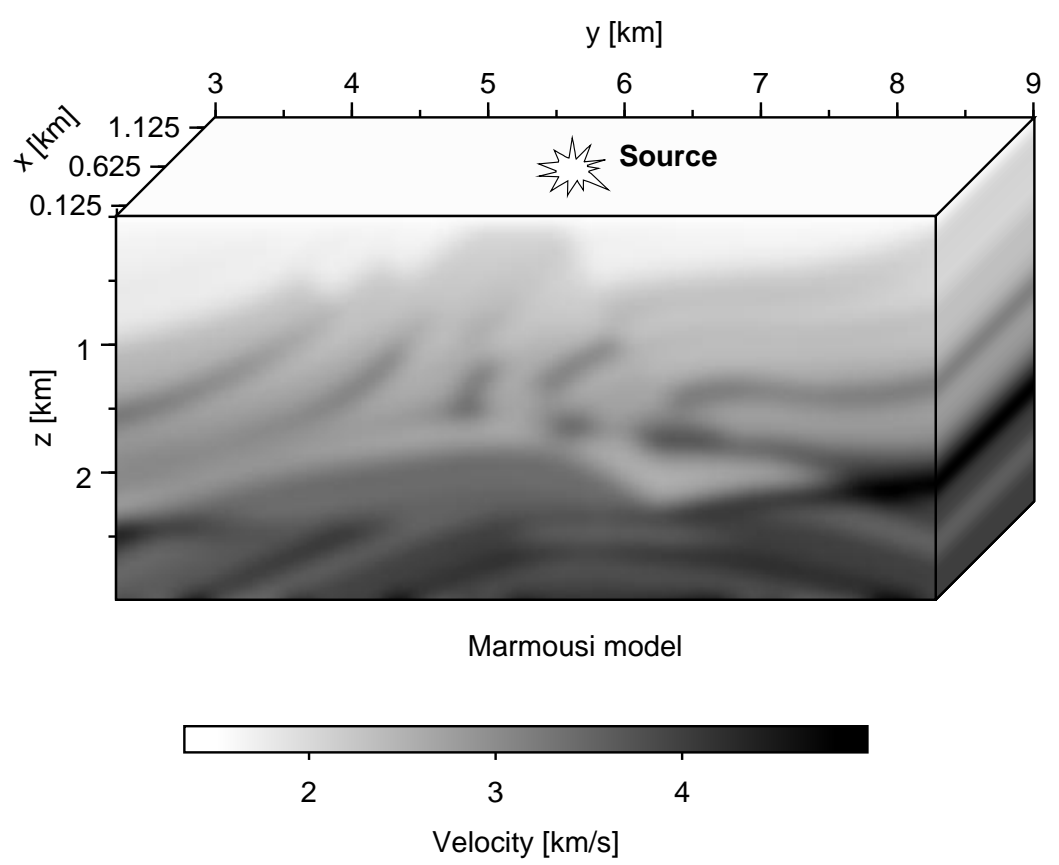

Figure 3.10: Marmousi model extended to three dimensions. The model was 100-fold smoothed. Only a part of the original model was used. The grid spacing is $12.5 \mathrm{~m}$ in either direction. The source is placed on top of the model at $625 \mathrm{~m}$ in $x$ - and $6 \mathrm{~km}$ in $y$-direction.

Table 3.6: Errors for the Marmousi model with 125m coarse grid spacing excluding a layer of $62.5 \mathrm{~m}$ depth under the source. Maximum errors are associated with triplications of the wavefront and therefore do not represent the method's accuracy.

\begin{tabular}{|lr|rrrr|}
\hline Interpolation & $\begin{array}{r}\text { Source shift } \\
\Delta s_{x}=\Delta s_{y}\end{array}$ & $\begin{array}{r}\text { Median of } \\
\text { rel. error }\end{array}$ & $\begin{array}{r}\text { Maximum } \\
\text { rel. error }\end{array}$ & $\begin{array}{r}\text { Median of } \\
\text { abs. error }\end{array}$ & $\begin{array}{r}\text { Maximum } \\
\text { abs. error }\end{array}$ \\
\hline hyperbolic & $0 \mathrm{~m}$ & $0.025 \%$ & $7.04 \%$ & $0.214 \mathrm{~ms}$ & $14.3 \mathrm{~ms}$ \\
hyperbolic & $50 \mathrm{~m}$ & $0.033 \%$ & $45.1 \%$ & $0.279 \mathrm{~ms}$ & $301 \mathrm{~ms}$ \\
parabolic & $0 \mathrm{~m}$ & $0.026 \%$ & $21.3 \%$ & $0.223 \mathrm{~ms}$ & $14.3 \mathrm{~ms}$ \\
parabolic & $50 \mathrm{~m}$ & $0.035 \%$ & $62.4 \%$ & $0.299 \mathrm{~ms}$ & $308 \mathrm{~ms}$ \\
trilinear & $0 \mathrm{~m}$ & $0.087 \%$ & $32.6 \%$ & $0.833 \mathrm{~ms}$ & $18.6 \mathrm{~ms}$ \\
\hline
\end{tabular}

In this example there are high maximum errors. These are no measure for the accuracy 


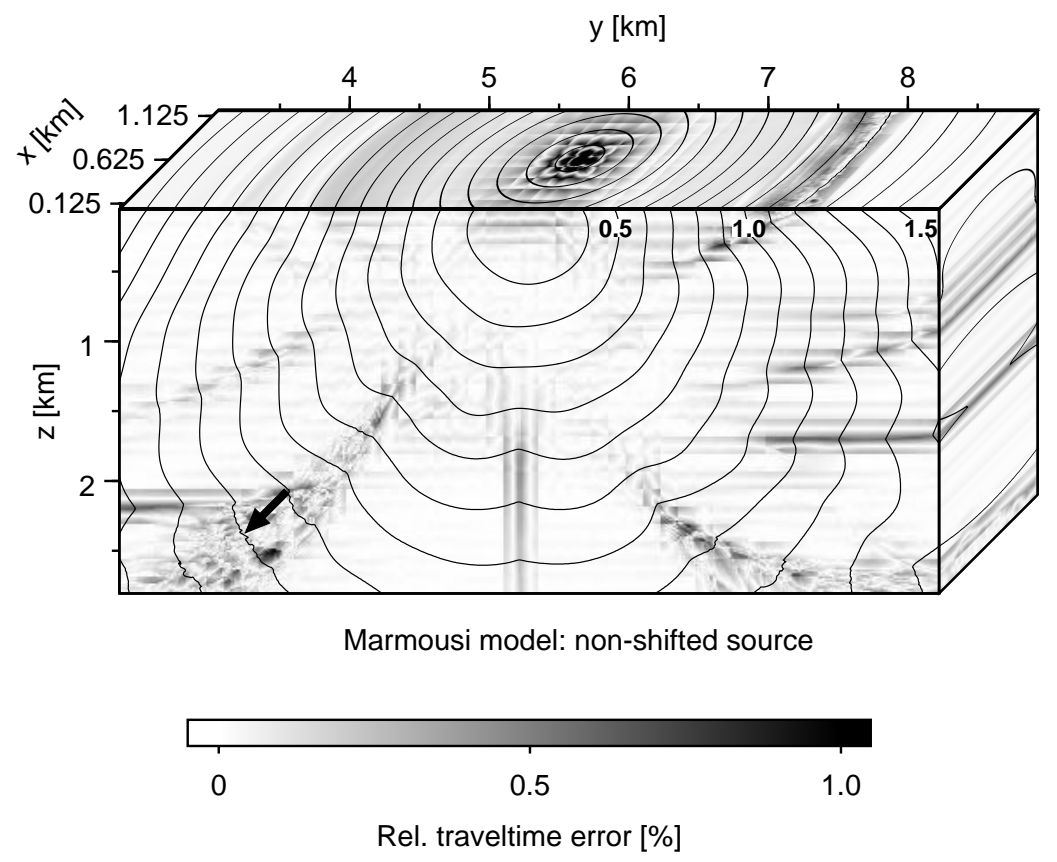

Figure 3.11: Relative traveltime errors for the Marmousi model using hyperbolic interpolation. Isochrones are given in seconds. The correlation of errors and "kinks" in the isochrones is clearly visible. The arrow at the $1.2 \mathrm{~s}$ isochrone in the lower left region indicates a higher error area that is caused by bad quality of the input traveltimes (due to a deficiency of the FD implementation used). This can be compensated by smoothing the input traveltimes.

of the interpolation scheme because they only occur in the vicinity of "kinks" in the isochrones. These indicate triplications of the wavefronts. The resulting errors are no surprise, since the assumption of smooth traveltimes is not valid anymore: a triplication consists of wavefronts belonging to two different branches. These must be interpolated separately. The obvious solution to overcome this problem is to employ later arrivals in the input traveltime scheme and to apply the interpolation to first and later arrivals separately. If traveltimes are computed, e.g., by the method of Coman and Gajewski (2001), which outputs traveltime tables sorted for different arrivals, the identification and interpolation of separate branches is only a matter of implementation. On the other hand, the method presented here itself provides means to detect triplications. This will be addressed below in Section 3.6.

Compared to the generic models, both interpolations for the Marmousi model yield similar quality. One reason is that for this model errors due to the different algorithms are dominated by errors caused by the quality of the input data, i.e. insufficient accuracy and particularly smoothness of the input traveltimes (see Figure 3.11). The fact that all wavelengths below $667 \mathrm{~m}$ were removed from the velocity model does not mean that a coarse grid spacing for the input traveltimes of $667 \mathrm{~m}$ will be sufficient. The required grid spacing for the traveltime interpolation depends on the wavenumbers in the input 


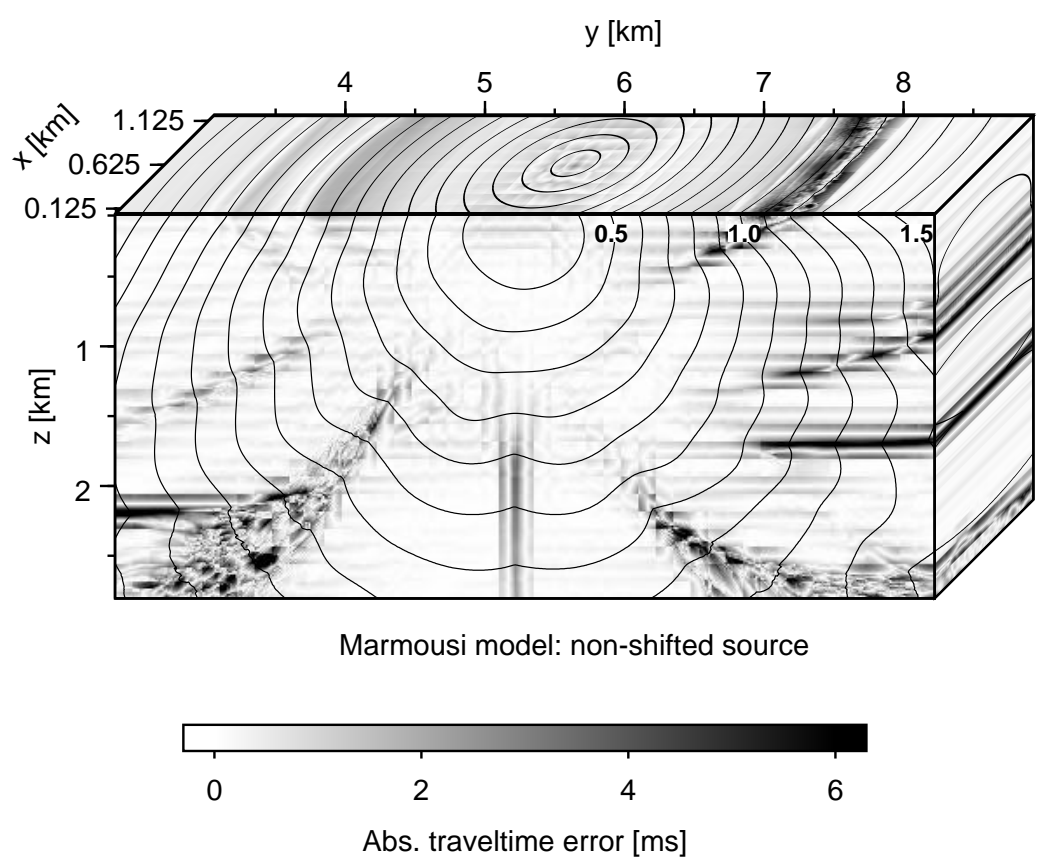

Figure 3.12: Absolute errors for the hyperbolic interpolation on the Marmousi model. Apart from regions in the vicinity of "kinks" in the wavefronts and areas with corrupted reference traveltimes (see Figure 3.11) the errors are well below a millisecond.

traveltimes, not the velocity model. Smoothing of the velocities is required for traveltime generators based on the ray method. This aspect will also be addressed in Section 6.8. 

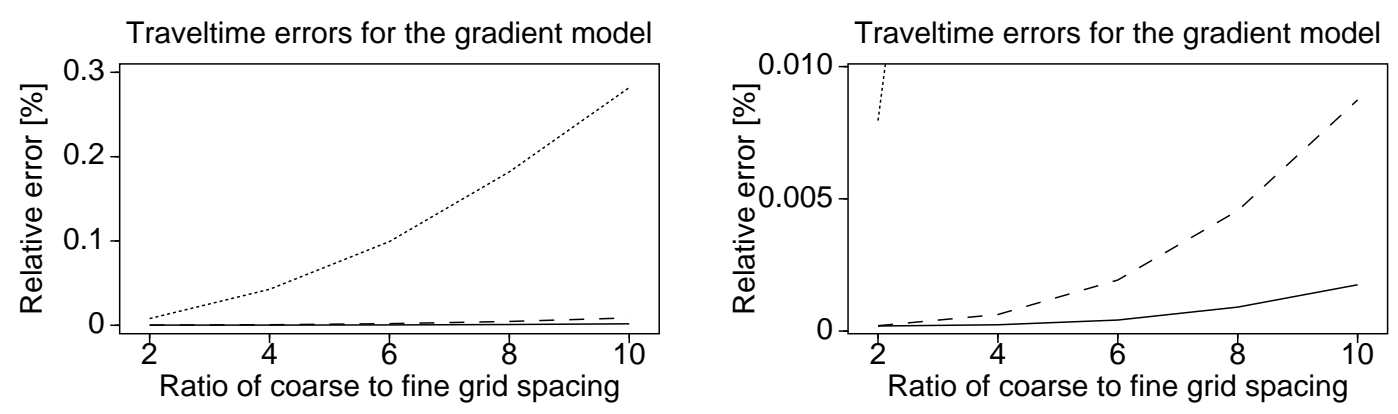

Figure 3.13: Relative traveltime errors vs. ratio of fine to coarse grid spacing for a constant velocity gradient model shown for trilinear (dotted line) parabolic (dashed line) and hyperbolic interpolation (solid line). The plot is displayed twice using different scales to illustrate the differences between trilinear and second-order interpolations (left), and between parabolic and hyperbolic interpolation (right).

\subsection{Influence of the Grid spacing}

The constant velocity gradient model with $V_{s}=3000 \mathrm{~m} / \mathrm{s}$ (see above) was further used to investigate the influence of the coarse grid spacing on the accuracy. Traveltime interpolation was carried out for coarse grid spacings ranging from 20 to $100 \mathrm{~m}$ using hyperbolic, parabolic, and trilinear interpolation. The fine grid spacing remained fixed at $10 \mathrm{~m}$. The resulting errors are displayed in Figure 3.13. I found the same quality relation between the three interpolation schemes as before and, as expected, the accuracy increasing for smaller coarse to fine grid ratio.

It must, however, be taken into account that the finer the coarse grid is made, the smaller the move-out becomes. This means that for very small grids the difference in traveltimes may be contaminated by round-off errors and the accuracy of the coefficients may degrade. This problem may also occur for very large distances to the source or any other situation where small move-out occurs. 


\subsection{Triplications}

For the derivation of the traveltime expansions (3.1.4) and (3.2.2), I have assumed that the traveltime fields under consideration are smooth with continuous first and second order derivatives. This is not always the case. In this section a method is proposed to detect regions where discontinuities arise.

Figure 3.14 shows a section of a wavefront with a triplication. At the meeting point $P$ of the two different branches it has a singularity. The slowness vector - the direction of the ray - is not defined at the point $P$. This makes it impossible to fit a traveltime for the surrounding area. If, however, these points are known and multi-valued traveltimes are available, coefficients can be determined by treating the ray branches separately. A method to detect these points can be easily derived by analysing the problem again, this time in terms of traveltimes. Figure 3.15 shows a very similar situation as Figure 3.14. Figure 3.15 is a multi-valued traveltime curve that the triplicated wavefront translates into. In the region between the grid points $x_{2}$ and $x_{3}$ the curve appears to be concave in difference to its otherwise convex behaviour, indicating that the sign of the curvature in this region would be determined in a wrong way.

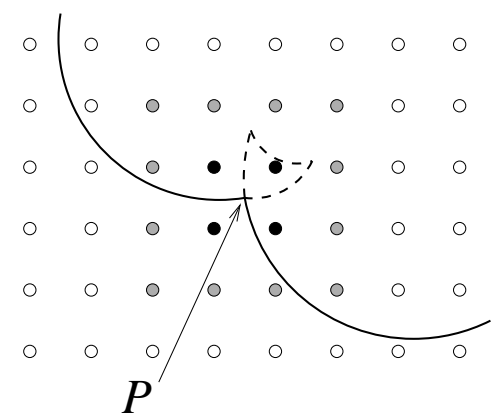

Figure 3.14: Triplication of a wavefront. Grid points surrounding the discontinuity are marked with black dots: here the traveltime field is multi-valued. This leads to wrong coefficients at grid points marked grey if only first arrivals are used for their determination. White grid points are not affected.

Figure 3.16 displays isochrones for a 2-D version of the Marmousi model (see Figure 3.10 ) and the negative values of the second order derivative matrix elements $G_{x x}$ and $G_{z z}$. The coincidence between the triplications and the negative curvature is obvious (although only first arrivals are shown, I will further use the term triplications for these discontinuities). Figure 3.16 also shows that in regions where the wavefront has a more horizontal orientation $G_{x x}$ is more suitable for identification of triplications, whereas for vertical wavefronts the behaviour of $G_{z z}$ is the better indicator. Therefore, the decision whether to investigate $G_{x x}$ or $G_{z z}$ is made automatically from the direction of the associated ray, i.e. the slowness vector, since it is perpendicular to the wavefront. The slowness is not only taken from a single point, but also from its vicinity as it would not yield sensible results at a triplication. In the 2-D case, this would mean that for $q_{0_{z}}>q_{0_{x}}$ the matrix element $G_{x x}$ will be taken and vice versa. However, the sign of $G$ alone is 


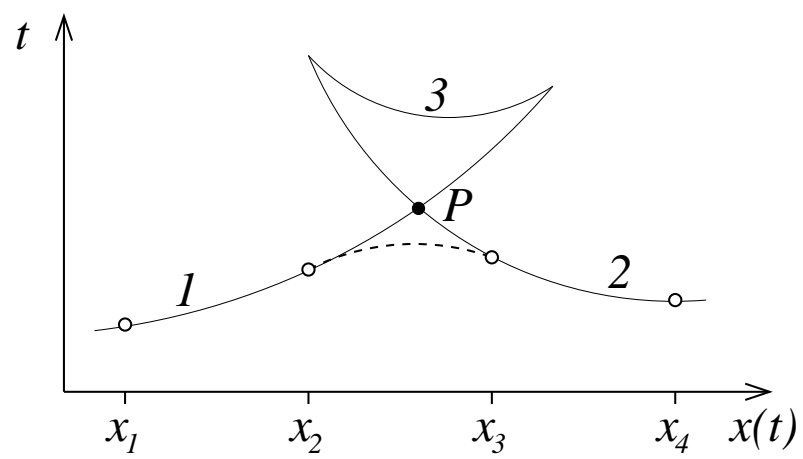

Figure 3.15: Triplication of a traveltime curve. The branches 1 and 2 meet at point $P$. First arrivals only map the curve below $P$. If the traveltimes are given on a grid as indicated by the white dots, the curvature between points $x_{2}$ and $x_{3}$ would appear to undergo a change in sign (dashed line).

not enough for a reliable triplication detection algorithm. For example, in the vicinity of near plane wavefronts the curvature becomes very small and $G$ may undergo a change in sign there.

Therefore, a second criterion is introduced: if, for example, $G$ would have positive sign, the first criterion would detect all points with negative signs. Only if the value of $G$ at one of these points is significantly more negative than in its surroundings, the point is selected. This second criterion is evaluated in terms of the quartiles ${ }^{2}$ of eight values surrounding the point under consideration, using in 2-D a box of 5 points width and 3 points height for $G_{x x}$ and $3 \times 5$ points for $G_{z z}$. Let $q_{i}$ denote the $i$-th quartile. Then a triplication will show by

$$
G+\kappa\left(q_{3}-q_{1}\right)<q_{2}
$$

where I have found the value of $\kappa=0.74$ to be a good choice. This value corresponds to the standard deviation, $\sigma$, of a Gaussian error distribution. If $\kappa$ is chosen too small, it would not distinguish between triplications and near plane wavefronts and too large $\kappa$ makes the algorithm too insensitive. The results for the Marmousi model with $\kappa=0.74$ are given in Figure 3.16 (bottom).

\footnotetext{
${ }^{2}$ as the median of a distribution divides it into two parts, where the number of elements with a value higher than the median equals the number of elements with a value smaller than the median, the quartiles divide the distribution into four parts. The second quartile equals the median.
} 


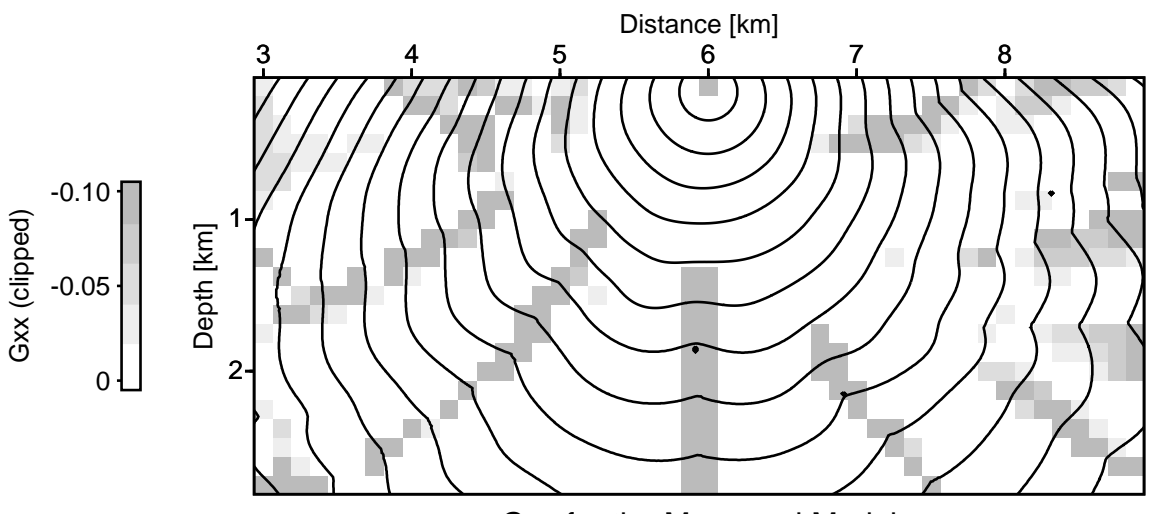

Gxx for the Marmousi Model

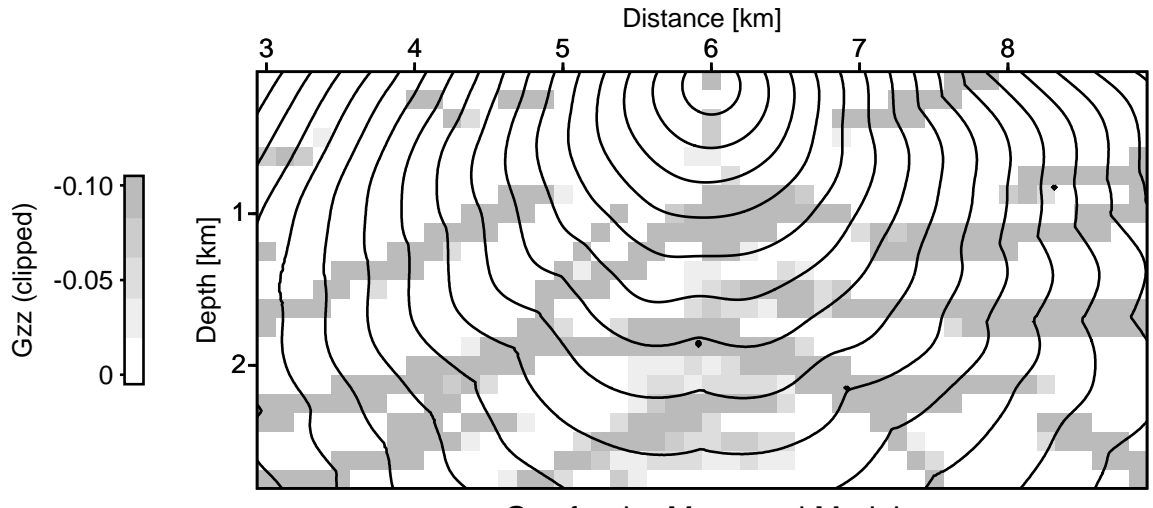

Gzz for the Marmousi Model

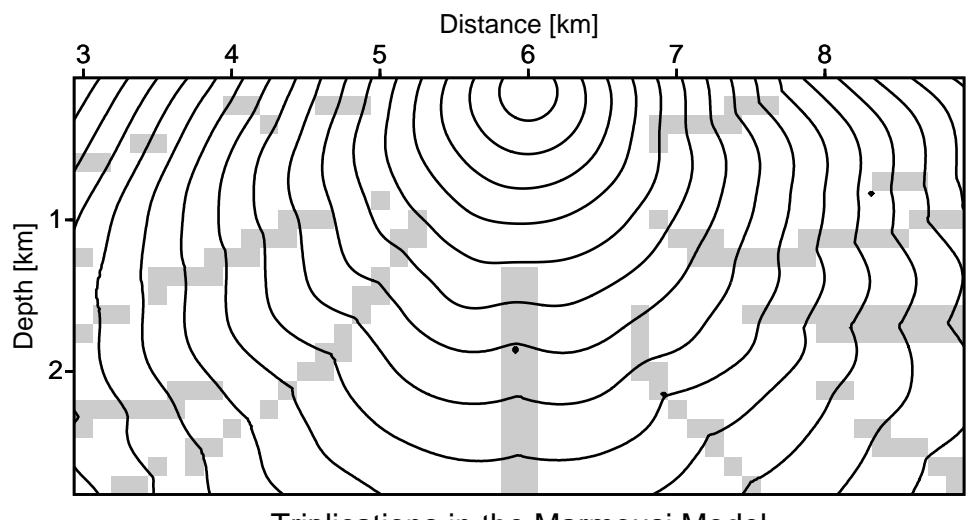

Triplications in the Marmousi Model

Figure 3.16: Matrix elements $G_{x x}$ (top) and $G_{z z}$ (middle) for the Marmousi model. Only negative values are displayed. They follow the triplications. Bottom: Triplications found by applying the quartile criterion in addition to the signs of $G$. 


\section{Chapter 4}

\section{A $6 \times 6$ Propagator Matrix?}

This chapter introduces a $6 \times 6$ matrix $\hat{\underline{T}}$ that is formed by the matrices $\underline{\hat{S}}, \underline{\hat{G}}$, and $\underline{\hat{N}}$ introduced in Chapter 3. Although $\hat{\underline{\mathbf{I}}}$ bears a strong similarity to the $4 \times 4$ Bortfeld propagator matrix T that was introduced in Section 2.9, it is not a propagator matrix, i.e. the solution to a differential equation like (2.6.2) under additional conditions (see Section 2.6). The matrix $\hat{\underline{T}}$ is useful, because its relation to $\underline{\underline{T}}$ links the $3 \times 3$ matrices $\underline{\hat{S}}, \underline{\hat{G}}$, and $\underline{\hat{N}}$ to the $2 \times 2$ matrices $\underline{A}, \underline{B}, \underline{C}$, and $\underline{D}$. The latter build the Bortfeld propagator $\underline{=}$ which is defined in reference surfaces, e.g., the reflector or registration surface. With $\underline{\underline{\mathbf{I}}}$, it is possible to express certain properties, e.g., the geometrical spreading of a reflected event, or a traveltime expansion into the reflector surface, directly in terms of the matrices $\underline{\hat{S}}$, $\underline{\hat{\mathrm{G}}}$, and $\underline{\hat{N}}$. This requires the relationship between $\underline{\underline{\mathbf{T}}}$ and $\underline{\underline{T}}$ which I will derive. It leads to another, useful notation for the submatrices of $\underline{\underline{T}}$.

I will introduce the $6 \times 6$ matrix $\hat{\underline{T}}$ in the first section of this chapter. The next section deals with the relationship between the matrix $\underline{\underline{\underline{T}}}$ and the propagator $\underline{\underline{T}}$. This relationship comprises the rotation of the $3 \times 3$ matrices involved in $\hat{\underline{I}}$ into the tangent planes of the surfaces in which $\underline{T}$ is defined. The curvature of these surfaces is also acknowledged in the relations given in this section. Finally, the last section treats reflected events in terms of propagator matrices, which leads to an expression for paraxial reflection traveltimes. The matrices in this expression can be decomposed into those of the two ray branches that build the reflected ray.

\subsection{The Matrix $\underline{\underline{T}}$}

The matrix $\hat{\underline{T}}$ negotiates between changes in positions and changes in slownesses, similar as the propagator $\underline{\underline{T}}$, but for 3 -D vectors. The slownesses are determined by taking the gradient with respect to source and receiver coordinates, respectively, of the parabolic traveltime expansion, Equation (3.1.4):

$$
\tau(\hat{\boldsymbol{s}}, \hat{\boldsymbol{g}})=\tau_{0}+\hat{\boldsymbol{q}}_{0} \Delta \hat{\boldsymbol{g}}-\hat{\boldsymbol{p}}_{0} \Delta \hat{\boldsymbol{s}}-\Delta \hat{\boldsymbol{s}}^{\top} \underline{\hat{\mathrm{N}}} \Delta \hat{\boldsymbol{g}}+\frac{1}{2} \Delta \hat{\boldsymbol{g}}^{\top} \underline{\hat{\mathrm{G}}} \Delta \hat{\boldsymbol{g}}-\frac{1}{2} \Delta \hat{\boldsymbol{s}}^{\top} \underline{\hat{\mathrm{S}}} \Delta \hat{\boldsymbol{s}}
$$


The slowness at the source is

$$
\hat{\boldsymbol{p}}=-\vec{\nabla}_{s} \tau=\hat{\boldsymbol{p}}_{0}+\underline{\hat{\mathrm{S}}} \Delta \hat{\boldsymbol{s}}+\underline{\hat{\mathrm{N}}} \Delta \hat{\boldsymbol{g}}
$$

and the slowness at the receiver is

$$
\hat{\boldsymbol{q}}=\vec{\nabla}_{g} \tau=\hat{\boldsymbol{q}}_{0}+\underline{\hat{\mathrm{G}}} \Delta \hat{\boldsymbol{g}}-\underline{\hat{\mathrm{N}}}^{\top} \Delta \hat{\boldsymbol{s}}
$$

Rearranging of Equations (4.1.2) and (4.1.3) gives

$$
\left(\begin{array}{c}
\Delta \hat{\boldsymbol{g}} \\
\hat{\boldsymbol{q}}-\hat{\boldsymbol{q}}_{0}
\end{array}\right)=\left(\begin{array}{cc}
\underline{\hat{\mathrm{N}}}^{-1} \underline{\hat{\mathrm{S}}}^{-1} & \underline{\hat{\mathrm{N}}}^{-1} \\
-\underline{\hat{\mathrm{N}}}^{\top}-\underline{\hat{\mathrm{G}}} \underline{\hat{\mathrm{N}}}^{-1} \underline{\hat{\mathrm{S}}} & \underline{\hat{\mathrm{G}}}^{-1} \underline{\mathrm{N}}^{-1}
\end{array}\right)\left(\begin{array}{c}
\Delta \hat{\boldsymbol{s}} \\
\hat{\boldsymbol{p}}-\hat{\boldsymbol{p}}_{0}
\end{array}\right) .
$$

I define the matrix $\underline{\underline{T}}$ as

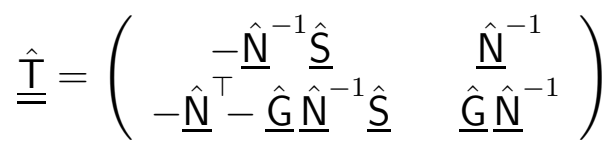

The next section will establish the relationship between the $6 \times 6$ matrix $\hat{\underline{T}}$ and the $4 \times 4$ propagator matrix $\underline{\underline{T}}$.

\subsection{Curved Surfaces}

The matrices $\underline{T}$ and $\hat{\underline{T}}$ have some common properties. In this section I introduce the $2 \times 2$ matrices $\underline{\tilde{G}}, \underline{\tilde{S}}$, and $\underline{\tilde{N}}$ and write the propagator $\underline{\underline{T}}$ in terms of these matrices, similar as $\underline{\underline{\mathbf{T}}}$ is formed by $\underline{\underline{G}}, \underline{\hat{S}}$, and $\underline{\hat{N}}$ :

$$
\underline{=}=\left(\begin{array}{cc}
-\tilde{\tilde{N}}^{-1} \underline{\tilde{S}}^{-} & \underline{\tilde{N}}^{-1} \\
-\underline{\tilde{N}}^{\top}-\underline{\tilde{G}} \underline{\tilde{N}}^{-1} \underline{\tilde{S}} & \underline{\tilde{G}}^{-1} \underline{\tilde{N}}^{-1}
\end{array}\right)
$$

Remembering from Chapter 2 that

$$
\stackrel{\mathrm{T}}{=}=\left(\begin{array}{ll}
\underline{\mathrm{A}} & \underline{\mathrm{B}} \\
\underline{\mathrm{C}} & \underline{\mathrm{D}}
\end{array}\right)
$$

the relationship between the matrices $\underline{\tilde{G}}, \underline{\tilde{S}}, \underline{\tilde{N}}$ and $\underline{A}, \underline{B}, \underline{C}, \underline{D}$ is

$$
\begin{aligned}
& \underline{\mathrm{A}}=-\underline{\tilde{\mathrm{N}}}^{-1} \underline{\tilde{\mathrm{S}}}, \\
& \underline{\mathrm{B}}=\underline{\tilde{\mathrm{N}}}^{-1}, \\
& \underline{\mathrm{C}}=-\underline{\tilde{\mathrm{N}}}^{\top}-\underline{\tilde{\mathrm{G}}}^{-1} \underline{\tilde{\mathrm{N}}}^{-1} \underline{\tilde{\mathrm{S}}}, \\
& \underline{\mathrm{D}}=\underline{\tilde{\mathrm{G}}} \underline{\tilde{N}}^{-1},
\end{aligned}
$$

and

$$
\begin{aligned}
& \underline{\tilde{\tilde{S}}}=-\underline{\mathrm{B}}^{-1} \underline{\mathrm{A}}, \\
& \underline{\tilde{\mathrm{G}}}=\underline{\mathrm{D}} \underline{\mathrm{B}}^{-1}, \\
& \underline{\tilde{\mathrm{N}}}=\underline{\mathrm{B}}^{-1} .
\end{aligned}
$$


Here, I have also used the fact that $\underline{\underline{T}}$ must obey the symplecticity relation $(2.6 .12)$. The matrix $\underline{\underline{\mathbf{T}}}$ is also a symplectic matrix.

To find a relationship between $\underline{\tilde{G}}, \underline{\tilde{S}}, \underline{\tilde{N}}$ and $\underline{\hat{G}}, \underline{\hat{S}}, \underline{\hat{N}}$, I use Equation (4.1.1), the parabolic traveltime expansion in the Cartesian coordinate system in which the traveltime tables are given. Since the traveltimes must not depend on the coordinate system, I can use a similar traveltime expansion in a coordinate system that coincides with the system in which the propagator $I$ is defined by the anterior and posterior surfaces. These correspond to the reflector's tangent plane and the registration surface and that system is denoted with a tilde * The according $3-\mathrm{D}$ vectors carry an additional hat. In this coordinate system associated with the surfaces, the traveltime expansion reads

$$
\tau(\hat{\tilde{\boldsymbol{s}}}, \hat{\tilde{\boldsymbol{g}}})=\tau_{0}+\hat{\tilde{\boldsymbol{q}}}_{0} \Delta \hat{\tilde{\boldsymbol{g}}}-\hat{\tilde{\boldsymbol{p}}}_{0} \Delta \hat{\tilde{\boldsymbol{s}}}-\Delta \hat{\tilde{\boldsymbol{s}}}^{\top} \underline{\tilde{\tilde{N}}} \Delta \hat{\tilde{\boldsymbol{g}}}+\frac{1}{2} \Delta \hat{\tilde{\boldsymbol{g}}}^{\top} \underline{\tilde{\tilde{G}}} \Delta \hat{\tilde{\boldsymbol{g}}}-\frac{1}{2} \Delta \hat{\tilde{\boldsymbol{s}}}^{\top} \underline{\tilde{\tilde{S}}} \Delta \hat{\tilde{\boldsymbol{s}}} .
$$

The anterior surface coordinate system has the 1- and 2-axes in the surface's tangent plane at $\hat{\tilde{\boldsymbol{s}}}_{0}=\hat{\boldsymbol{s}}_{0}$ and the 3 -axis perpendicular to it. The vector $\Delta \hat{\tilde{\boldsymbol{s}}}=\left(\Delta \tilde{\boldsymbol{s}}, \Delta \tilde{s}_{3}\right)^{\top}=$ $\left(\Delta \tilde{s}_{1}, \Delta \tilde{s}_{2}, \Delta \tilde{s}_{3}\right)^{\top}$ describes a point on the anterior surface. Its 3 -component is given by

$$
\Delta \tilde{s}_{3}=\frac{1}{2} \Delta \tilde{\boldsymbol{s}}^{\top} \underline{\mathrm{F}}_{s} \Delta \tilde{\boldsymbol{s}}
$$

where the $2 \times 2$ matrix $\underline{F}_{s}$ is the curvature matrix of the anterior surface. It is zero for a plane, therefore the 3 -component vanishes in this case. The 2 -D vector $\Delta \tilde{\boldsymbol{s}}$ is computed by

$$
\Delta \tilde{\boldsymbol{s}}=\underline{1}_{2 \times 3} \underline{\hat{Z}}_{s} \Delta \hat{\boldsymbol{s}}
$$

where the matrix $\underline{1}_{2 \times 3}=\underline{1}_{3 \times 2}^{\top}$ is

$$
\underline{1}_{2 \times 3}=\left(\begin{array}{ccc}
1 & 0 & 0 \\
0 & 1 & 0
\end{array}\right)
$$

The matrix $\hat{Z}_{s}$ in Equation (4.2.12) describes the rotation into the tangent plane of the anterior surface. It is defined corresponding to Equation (2.8.1),

$$
Z_{i j}=\frac{\partial x_{i}}{\partial \tilde{x}_{j}}=\frac{\partial \tilde{x}_{j}}{\partial x_{i}}=\hat{\overrightarrow{\boldsymbol{x}}}_{i} \cdot \hat{\overrightarrow{\boldsymbol{x}}}_{j}
$$

Vectors $\hat{\overrightarrow{\boldsymbol{x}}}_{i}$ are the base vectors of the global Cartesian coordinate system, $\hat{\overrightarrow{\overrightarrow{\boldsymbol{x}}}}_{i}\left(=\overrightarrow{\boldsymbol{\boldsymbol { \imath }}}_{i}\right)$ are those of the interface coordinates. It follows that the 1-component of the vector $\tilde{\boldsymbol{s}}$ lies in the plane defined by the emerging wave and the anterior surface (see Section 2.8). These considerations also apply to the posterior surface with the appropriate index $g$ instead of $s$. Figure 4.1 gives an example where the 1-components of the two systems coincide and the angle $\alpha$ lies between the 3 -components. For this example the rotation matrix $\underline{\underline{Z}}$ becomes

$$
\hat{\mathbf{Z}}=\left(\begin{array}{ccc}
1 & 0 & 0 \\
0 & \cos \alpha & \sin \alpha \\
0 & -\sin \alpha & \cos \alpha
\end{array}\right)
$$




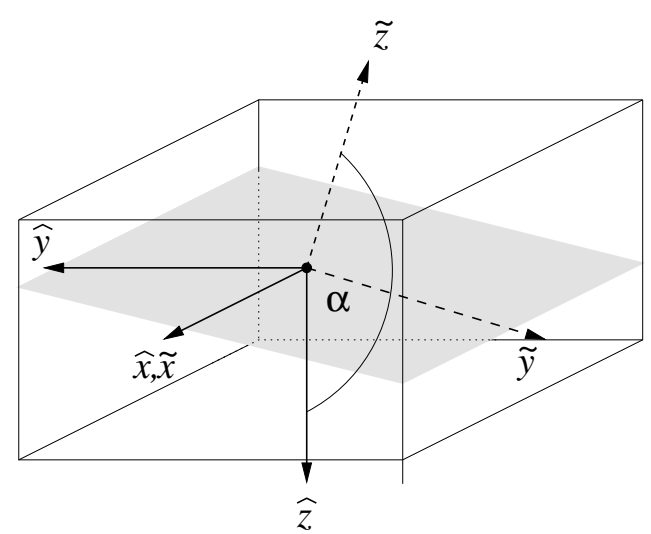

Figure 4.1: Rotation of 3-D second-order derivative matrices into the anterior surface's tangent plane: the coordinate system of the anterior surface (gray) is denoted by a tilde . The Cartesian system that coincides with the system in which the traveltime tables are given is indicated by a hat . The angle $\alpha$ is the angle between the 3 -axes $(z)$ of both systems. In this plot the 1-axes $(x)$ of both systems coincide and the curvature of the anterior surface is zero. The rotation matrix for this case is given in the text. (The index $s$ was omitted in the figure.)

Since the traveltime must not depend on the coordinate system, expressions (4.1.1) and (4.2.10) must be equal for points in the corresponding surfaces. Applying Equations (4.2.11) and (4.2.12) for both anterior and posterior surface, and retaining only terms up to second order yields

$$
\tau(\tilde{\boldsymbol{s}}, \tilde{\boldsymbol{g}})=\tau_{0}+\tilde{\boldsymbol{q}}_{0} \Delta \tilde{\boldsymbol{g}}-\tilde{\boldsymbol{p}}_{0} \Delta \tilde{\boldsymbol{s}}-\Delta \tilde{\boldsymbol{s}}^{\top} \underline{\tilde{\mathbf{N}}} \Delta \tilde{\boldsymbol{g}}+\frac{1}{2} \Delta \tilde{\boldsymbol{g}}^{\top} \underline{\tilde{\mathrm{G}}} \Delta \tilde{\boldsymbol{g}}-\frac{1}{2} \Delta \tilde{\boldsymbol{s}}^{\top} \underline{\tilde{\mathbf{S}}} \Delta \tilde{\boldsymbol{\boldsymbol { s }}}
$$

The $2 \times 2$ matrices $\underline{\tilde{N}}, \underline{\tilde{G}}$, and $\underline{\tilde{S}}$ are the same as in Equation (4.2.8) and are computed from the matrices $\underline{\hat{N}}, \underline{\hat{G}}$, and $\underline{\hat{S}}$ as follows:

$$
\begin{aligned}
& \underline{\tilde{N}}=\underline{1}_{2 \times 3} \underline{\hat{Z}}_{s}^{\top} \hat{\mathbf{N}} \underline{\mathbf{Z}}_{g} \underline{1}_{3 \times 2} \quad, \\
& \underline{\tilde{S}}=\underline{1}_{2 \times 3} \underline{\hat{Z}}_{s}^{\top} \hat{\hat{S}} \underline{\hat{Z}}_{s} \underline{1}_{3 \times 2}+\frac{\cos \theta_{s}}{v_{s}} \underline{\mathrm{F}}_{s} \text {, } \\
& \underline{\tilde{\mathrm{G}}}=\underline{1}_{2 \times 3} \underline{\hat{\mathrm{Z}}}_{g}^{\top} \underline{\hat{\mathrm{G}}}_{\hat{\mathrm{Z}}_{g}} \underline{1}_{3 \times 2}+\frac{\cos \theta_{g}}{v_{g}} \underline{\mathrm{F}}_{g} \text {. }
\end{aligned}
$$

The angle $\theta_{g}$ is the incidence angle at the posterior surface at $\hat{\tilde{\boldsymbol{g}}}_{0}=\hat{\boldsymbol{g}}_{0}$ and $\theta_{s}$ is the emergence angle at the anterior surface at $\hat{\tilde{\boldsymbol{s}}}_{0}=\hat{\boldsymbol{s}}_{0}$. The quantity $\cos \theta_{s} / v_{s}$ is the 3component of the slowness vector in the anterior surface coordinate system (accordingly for $\left.\cos \theta_{g} / v_{g}\right)$. The 2 -D slowness vectors in Equation (4.2.16), $\tilde{\boldsymbol{p}}_{0}$ and $\tilde{\boldsymbol{q}}_{0}$, are given by

$$
\begin{aligned}
& \tilde{\boldsymbol{p}}_{0}=\underline{1}_{2 \times 3} \underline{\hat{Z}}_{s} \hat{\boldsymbol{p}}_{0}, \\
& \tilde{\boldsymbol{q}}_{0}=\underline{1}_{2 \times 3} \underline{\hat{z}}_{g} \hat{\boldsymbol{q}}_{0} .
\end{aligned}
$$


One useful application of the propagator $T$ is the decomposition of the ray that describes a reflected event into two individual ray $\overline{\bar{b}}$ ranches. This decomposition is demonstrated in the next section.

\subsection{Reflected Events}

For any point $M$ on a smooth interface along the central ray, the propagator $\underline{I}$ satisfies the following chain rule (Hubral et al., 1992b):

$$
\underline{\underline{\mathrm{T}}}\left(\tilde{\mathrm{g}}_{\mathbf{0}}, \tilde{\mathbf{s}}_{\mathbf{0}}\right)=\underline{\underline{\mathrm{T}}}\left(\tilde{\mathrm{g}}_{\mathbf{0}}, M\right) \cdot \underline{\underline{\mathrm{T}}}\left(M, \tilde{\mathbf{s}}_{\mathbf{0}}\right)=\underline{\underline{\mathrm{T}}}_{2} \cdot \underline{\mathrm{T}}_{1}
$$

where I denote the matrices from $\tilde{\mathbf{s}}_{\mathbf{0}}$ to $M$ with the index 1, i.e.

$$
\underline{\underline{I}}_{1}=\left(\begin{array}{cc}
-\tilde{\tilde{N}}_{1}^{-1} \tilde{\tilde{\mathrm{S}}}_{1} & \underline{\tilde{\mathbf{N}}}_{1}^{-1} \\
-\underline{\tilde{N}}_{1}^{\top}-\underline{\tilde{\mathrm{G}}}_{1} \underline{\tilde{N}}_{1}^{-1} \underline{\tilde{S}}_{1} & \underline{\tilde{G}}_{1} \underline{\tilde{N}}_{1}^{-1}
\end{array}\right) \text {. }
$$

For the ray from $M$ to $\tilde{\mathbf{g}}_{0}$ I use the reverse propagator $\underline{T}^{*}\left(M, \tilde{\mathbf{g}}_{\mathbf{0}}\right)$ for the reverse ray from $\tilde{\mathrm{g}}_{\mathbf{0}}$ to $M$ instead of $\underline{\underline{\mathrm{T}}}\left(\tilde{\mathrm{g}}_{\mathbf{0}}, M\right)$. The relationship between a propagator matrix and that of its reverse ray which is given by Hubral et al. (1992a) leads to

$$
\stackrel{\mathrm{T}}{=}_{2}=\left(\begin{array}{cc}
\underline{\tilde{\mathrm{N}}}_{2}^{-\top} \underline{\tilde{\mathrm{G}}}_{2} & \underline{\tilde{\mathrm{N}}}_{2}^{-\top} \\
-\underline{\tilde{\mathrm{N}}}_{2}-\underline{\tilde{\mathrm{S}}}_{2} \underline{\tilde{N}}_{2}^{-\top} \underline{\tilde{G}}_{2} & -\underline{\tilde{S}}_{2} \underline{\tilde{N}}_{2}^{-\top}
\end{array}\right) .
$$

Inserting these two propagators into Equation (4.3.19) yields the resulting matrices

$$
\begin{aligned}
& \underline{\tilde{\mathbf{N}}}=\underline{\tilde{N}}_{1}\left(\underline{\tilde{\mathrm{G}}}_{1}+\underline{\tilde{\mathrm{G}}}_{2}\right)^{-1} \underline{\tilde{\mathrm{N}}}_{2}^{\top} \\
& \underline{\tilde{\mathrm{G}}}=-\underline{\tilde{S}}_{2}-\underline{\tilde{N}}_{2}\left(\underline{\tilde{\mathrm{G}}}_{1}+\underline{\tilde{G}}_{2}\right)^{-1} \underline{\tilde{\mathrm{N}}}_{2}^{\top} \\
& \underline{\tilde{\mathrm{S}}}=\underline{\tilde{\mathrm{S}}}_{1}+\underline{\tilde{N}}_{1}\left(\underline{\tilde{\mathrm{G}}}_{1}+\underline{\tilde{\mathrm{G}}}_{2}\right)^{-1} \underline{\tilde{\mathrm{N}}}_{1}^{\top}
\end{aligned}
$$

which form $\underline{\underline{\mathrm{T}}}\left(\tilde{\mathrm{g}}_{0}, \tilde{\mathbf{s}}_{\mathbf{0}}\right)$.

If I assume a reflector at $M$, the resulting propagator $\underline{\underline{\mathrm{T}}}\left(\tilde{\mathrm{g}}_{\mathbf{0}}, \tilde{\mathbf{s}}_{\mathbf{0}}\right)$ leads to the paraxial reflection traveltime

$$
\tau_{R}(\tilde{\boldsymbol{s}}, \tilde{\boldsymbol{g}})=\tau_{0}+\tilde{\boldsymbol{q}}_{0} \Delta \tilde{\boldsymbol{g}}-\tilde{\boldsymbol{p}}_{0} \Delta \tilde{\boldsymbol{s}}-\Delta \tilde{\boldsymbol{s}}^{\top} \underline{\tilde{N}} \Delta \tilde{\boldsymbol{g}}+\frac{1}{2} \Delta \tilde{\boldsymbol{g}}^{\top} \underline{\tilde{G}} \Delta \tilde{\boldsymbol{g}}-\frac{1}{2} \Delta \tilde{\boldsymbol{s}}^{\top} \underline{\underline{S}} \Delta \tilde{\boldsymbol{s}}
$$

with the matrices $\underline{\tilde{N}}, \underline{\tilde{G}}$, and $\underline{\tilde{S}}$ given by Equation (4.3.22). 


\section{Chapter 5}

\section{Geometrical Spreading}

Geometrical spreading, together with traveltimes, plays an important role in many applications of reflection seismology, such as migration, tomography, and modelling. The traditional method of computing geometrical spreading is to perform dynamic ray tracing, as described by Červený et al. (1977) or Popov and Pšenčík (1978). Dynamic ray tracing, however, is a rather time consuming process. Therefore, other methods have been proposed. In 1990, Vidale and Houston suggested a technique to calculate amplitudes from traveltimes. Although it is very fast it suffers from problems with accuracy. The method proposed by VanTrier and Symes (1990) is based on a finite difference (FD) eikonal solver. An improved FD scheme that achieves higher order accuracy was suggested by Pusey and Vidale (1991). Another way to determine geometrical spreading is proposed by Buske (2000), by directly solving the transport equation with finite differences. All these techniques are more efficient than dynamic ray tracing. Lower order FD methods are, however, restricted to fine grids, whereas higher order schemes are less efficient. This restriction does not apply to the amplitude estimation using a wavefront construction method introduced by Vinje et al. (1993). Another ray-based approach is shown by Hubral et al. (1992a), who give an expression for the geometrical spreading that is directly related to second order traveltime derivatives in terms of the Bortfeld (1989) matrices.

The method of computing geometrical spreading that I suggest in this chapter is based on the traveltime coefficients introduced in Chapter 3, and closely related to the formula by Hubral et al. (1992a). The coefficient matrices that were introduced in Chapter 3

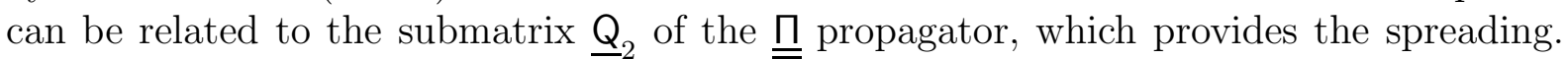
Therefore, the geometrical spreading can be determined from traveltimes. The resulting expression is equivalent to the result obtained by Hubral et al. (1992a).

After a short introduction to the cause of geometrical spreading I derive expressions for the spreading in terms of the second order traveltime derivative matrices. That section is followed by application to different velocity models, ranging from models, where the analytic solution is known to the highly complex Marmousi model. The spreading can be computed from both parabolic and hyperbolic traveltime coefficients. The results confirm again the superiority of the hyperbolic expression. 


\subsection{Introduction}

Conservation of energy is a fundamental rule that also applies to wave propagation. It means that, unless attenuation is caused by mechanisms like, e.g., absorption, the energy of a wavefront remains unchanged. These mechanisms are not considered in this chapter and I assume an ideal elastic medium. While the wave propagates through the medium, its initial energy is "spread" on the expanding wavefront, causing a decrease in the wave amplitude. This phenomenon is called geometrical spreading.

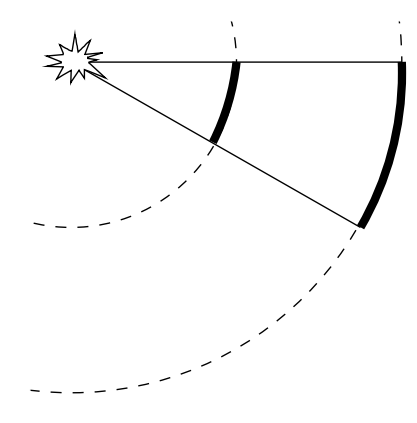

a)

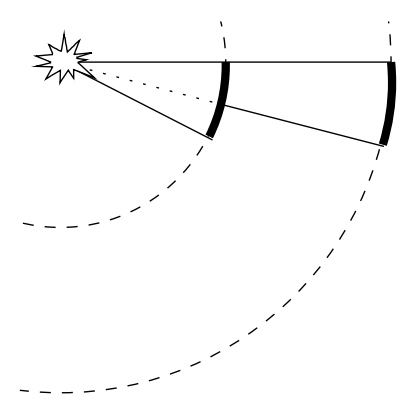

b)

Figure 5.1: Geometrical spreading for a point source in a homogeneous medium. The wavefronts are circles in 2-D or spheres in 3-D. The energy flux through a solid angle element remains constant (a). The flux through a surface element depends on the radius of curvature of the wavefront (b).

Figure 5.1 displays a point source in a homogeneous medium and two corresponding wavefronts, circles in 2-D and spheres in 3-D. Figure 5.1 a) states that the amount of energy in a section of the wavefront (expressed by the flux) remains constant for a fixed solid angle. This means, however, that for the second wavefront with smaller curvature the same amount of energy is spread over a larger surface than for the first wavefront with high curvature. Since a detector measures the energy flux through a surface element, not through a solid angle, it will register a smaller amplitude for the second wavefront than for the first one (see Figure 5.1, b). The ratio of the measuring surface element to the complete wavefront is proportional to $1 / r^{2}$ in $3-\mathrm{D}$, and $\propto 1 / r$ in $2-\mathrm{D}$, where $r$ is the radius of curvature of the wavefront. The amplitude corresponds to the square-root of the flux, therefore the amplitude of the wave in a 3-D medium decreases with $1 / r$ and with $1 / \sqrt{r}$ in 2 -D. The relative geometrical spreading $L$ is the inverse of the amplitude, thus $L \propto r$ in 3-D and $L \propto \sqrt{r}$ in 2-D. Although a homogeneous medium was chosen for the explanation, the curvature of the wavefront also determines the geometrical spreading in arbitrary media. Furthermore, Figure 5.2 shows that the measured flux also depends on the incidence angle between the wavefront and the registration surface.

Knowing that the geometrical spreading is governed by wavefront curvature and that the curvature of a function is determined by its second order derivatives, a relationship 


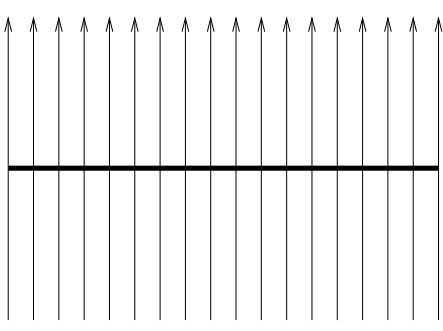

a)

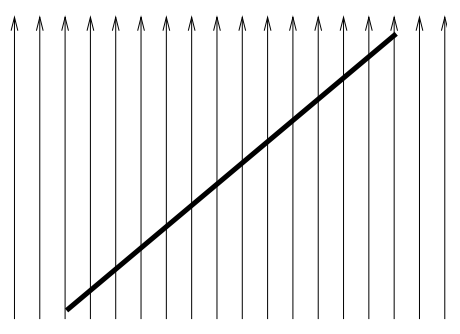

b)

Figure 5.2: Energy flux through a surface. The arrows denote the flux and the thick lines the measuring surfaces. Both surfaces have equal size. In the case of vertical incidence, a), more flux lines pass through the surface than for oblique incidence, b). Therefore, the measured amplitude depends on the incidence angle.

between the spreading and the traveltime derivatives from Chapter 3 can be established. An expression for the spreading in terms of second order traveltime derivatives in a specific, ray centred coordinate system was already given in Chapter 2. The following section will relate that result to the coefficients from the traveltime expansions in Chapter 3.

\section{$5.2 \quad$ Spreading and Traveltime Derivatives}

From Equation (2.5.15) I take the modulus of the relative geometrical spreading, $L=|\operatorname{det} \underline{\mathrm{Q}}|^{1 / 2}$. For a point source at $s_{0}$ and a receiver at $g_{0}$, Equation (2.6.18) with initial point source conditions (2.6.7) yields

$$
\left(\begin{array}{l}
\underline{\mathrm{Q}}\left(g_{0}\right) \\
\underline{\mathrm{P}}\left(g_{0}\right)
\end{array}\right)=\left(\begin{array}{ll}
\underline{\mathrm{Q}}_{1}\left(g_{0}, s_{0}\right) & \underline{\mathrm{Q}}_{2}\left(g_{0}, s_{0}\right) \\
\underline{\mathrm{P}}_{1}\left(g_{0}, s_{0}\right) & \underline{\mathrm{P}}_{2}\left(g_{0}, s_{0}\right)
\end{array}\right)\left(\begin{array}{l}
\underline{0} \\
\underline{1}
\end{array}\right)=\left(\begin{array}{l}
\underline{\mathrm{Q}}_{2}\left(g_{0}, s_{0}\right) \\
\underline{\mathrm{P}}_{2}\left(g_{0}, s_{0}\right)
\end{array}\right),
$$

and therefore

$$
L=\left|\operatorname{det} \underline{Q}_{2}\left(g_{0}, s_{0}\right)\right|^{1 / 2} .
$$

It is custom to use the normalised geometrical spreading $\mathcal{L}$ rather than the relative spreading $L$, which was introduced by Ursin (1990) to be

$$
\mathcal{L}\left(g_{0}, s_{0}\right)=\frac{1}{V_{s}} L\left(g_{0}, s_{0}\right)
$$

With $\underline{\mathrm{Q}}\left(g_{0}, s_{0}\right)=-\underline{\mathrm{Q}}^{\top}\left(s_{0}, g_{0}\right)$ the reciprocity relation for the normalised spreading reads

$$
L\left(g_{0}, s_{0}\right)=V_{s} \mathcal{L}\left(g_{0}, s_{0}\right)=V_{g} \mathcal{L}\left(s_{0}, g_{0}\right)=L\left(s_{0}, g_{0}\right)
$$

In Chapter 3, I have shown how the second order traveltime derivative matrices $\underline{\hat{G}}, \underline{\hat{S}}$, and $\underline{\hat{N}}$ can be determined from traveltime maps. Since $\underline{Q}_{2}$ is also a matrix of second order traveltime derivatives, a relationship can be established that links the matrix $\underline{\hat{N}}$ to $\underline{\mathrm{Q}}_{2}$. For this reason, I compare the paraxial traveltime approximation in Cartesian coordinates (2.7.16) to the parabolic traveltime expansion introduced in (3.1.4), leading to

$$
\underline{\hat{\mathrm{N}}}=\underline{\hat{\mathrm{H}}}_{s} \underline{\hat{\mathrm{Q}}}_{2}^{-} \underline{\hat{\mathrm{H}}}_{g}^{\top}
$$


where the matrices $\underline{\hat{H}}$ are the transformation matrices from Cartesian to ray centred coordinates on the central ray. This can be solved for $\underline{\mathrm{Q}}_{2}^{-}$, which I define as

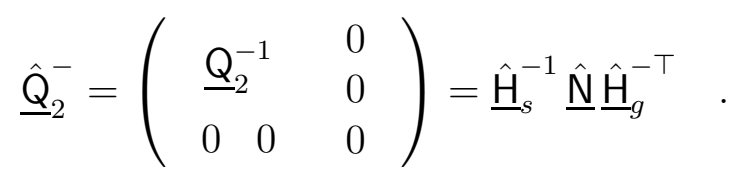

The matrices $\underline{\hat{\mathrm{H}}}_{s}$ and $\underline{\hat{\mathrm{H}}}_{g}$ are defined by Equation (2.5.15). Matrix $\underline{\hat{\mathrm{H}}}_{s}$ consists of the three column vectors $\overrightarrow{\boldsymbol{e}}_{1_{s}}, \overrightarrow{\boldsymbol{e}}_{2_{s}}$, and $\overrightarrow{\boldsymbol{e}}_{3_{s}}=\overrightarrow{\boldsymbol{t}}_{s}=V_{s} \hat{\boldsymbol{p}}_{0}$. The matrix $\underline{\hat{H}}_{g}$ is defined accordingly, but using the index $g$ instead of $s$ and $\hat{\boldsymbol{q}}_{0}$ instead of $\hat{\boldsymbol{p}}_{0}$. It can easily be shown that $\underline{\hat{H}}^{\top}=\underline{\hat{H}}^{-1}$. The slowness vectors in Cartesian coordinates (e.g., $\hat{\boldsymbol{p}}_{0}$ ) follow from Figure 5.3, and with the slowness vector in ray centred coordinates $\hat{\boldsymbol{p}}_{0}^{(q)}=\left(0,0,1 / V_{s}\right)^{\top}$

$$
\hat{\boldsymbol{p}}_{0}=\underline{\hat{\mathrm{H}}}\left(s_{0}\right) \hat{\boldsymbol{p}}_{0}^{(q)}=\left(\begin{array}{c}
-\frac{1}{V_{s}} \sin \vartheta_{s} \cos \varphi_{s} \\
\frac{1}{V_{s}} \sin \vartheta_{s} \sin \varphi_{s} \\
\frac{1}{V_{s}} \cos \vartheta_{s}
\end{array}\right)
$$

and $\hat{\boldsymbol{q}}_{0}$ accordingly.

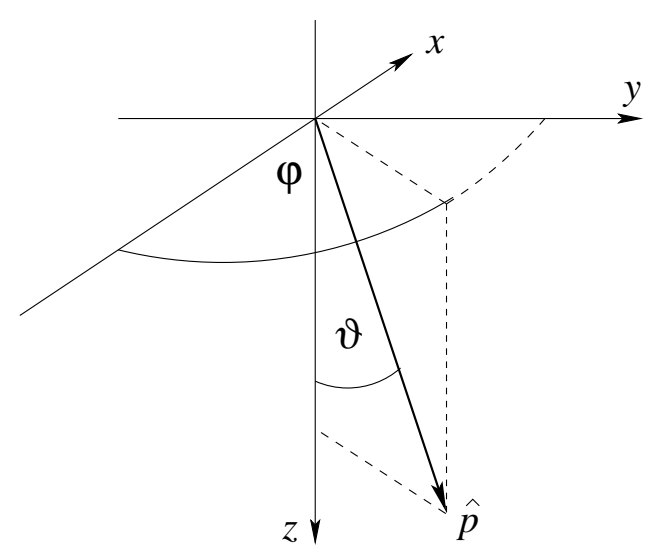

Figure 5.3: Slowness vector and angles in the Cartesian coordinate system. The angle $\vartheta$ is the azimuth, $\varphi$ the polar angle.

Now I use again the constraints on the matrix elements of $\underline{\hat{N}}$ given by the eikonal equations at the central source and receiver positions. They were already used to derive expressions for the $z$-components of $\underline{\hat{N}}$ and $\underline{\hat{S}}$ in Section 3.1. In addition to equations (3.1.13) and (3.1.14), I use the following relations

$$
\begin{aligned}
& N_{x z}=-\frac{q_{x_{0}}}{q_{z_{0}}} N_{x x}-\frac{q_{y_{0}}}{q_{z_{0}}} N_{x y}, \\
& N_{y z}=-\frac{q_{x_{0}}}{q_{z_{0}}} N_{y x}-\frac{q_{y_{0}}}{q_{z_{0}}} N_{y y}, \\
& N_{z z}=\frac{1}{p_{z_{0}} q_{z_{0}}}\left(p_{x_{0}} q_{x_{0}} N_{x x}+p_{x_{0}} q_{y_{0}} N_{x y}+p_{y_{0}} q_{x_{0}} N_{y x}+p_{y_{0}} q_{y_{0}} N_{y y}\right) .
\end{aligned}
$$


Equations (3.1.13), (3.1.14), and (5.2.8) can be rewritten to

$$
\begin{aligned}
N_{K 3} & =-\frac{H_{g_{I 3}}}{H_{g_{33}}} N_{K I} \\
N_{3 J} & =-\frac{H_{s_{I 3}}}{H_{s_{33}}} N_{I J} \\
N_{33} & =\frac{H_{s_{J 3}}}{H_{s_{33}}} \frac{H_{g_{K 3}}}{H_{g_{33}}} N_{J K}
\end{aligned}
$$

( $I, J, K=1,2$ and summation convention is applied). Furthermore, in component notation Equation (5.2.6) reads

$$
\begin{aligned}
Q_{2_{i l}}^{-} & =H_{s_{j i}} N_{j k} H_{g_{k l}} \\
& =\left(\frac{H_{s_{J i}} H_{s_{33}}-H_{s_{J 3}} H_{s_{3 i}}}{H_{s_{33}}}\right) N_{J K}\left(\frac{H_{g_{K l}} H_{g_{33}}-H_{g_{K 3}} H_{g_{3 l}}}{H_{g_{33}}}\right)
\end{aligned}
$$

$(i, j, k, l=1,2,3)$, where Equation (5.2.9) has been applied. From Equation (5.2.10) follows immediately that

$$
\begin{array}{ll}
Q_{2_{i 3}}^{-}=0 & (i=1,2,3) \\
Q_{2_{3 i}}^{-}=0 & (i=1,2,3)
\end{array} \quad
$$

For the remaining elements of $\underline{\underline{Q}}_{2}^{-}$I use the fact that the base vectors $\overrightarrow{\boldsymbol{e}}_{1}, \overrightarrow{\boldsymbol{e}}_{2}$, and $\overrightarrow{\boldsymbol{t}}$ are an orthonormal system with $\overrightarrow{\boldsymbol{e}}_{1} \times \overrightarrow{\boldsymbol{e}}_{2}=\overrightarrow{\boldsymbol{t}}$ etc. This leads to

$$
\frac{H_{J I} H_{33}-H_{J 3} H_{3 I}}{H_{33}}=H_{J I}^{-1}
$$

The element $H_{J I}^{-1}$ is the $J I^{\text {th }}$ element of the matrix $\underline{\mathrm{H}}^{-1}$, where

$$
\underline{\mathbf{H}}=\left(\begin{array}{ll}
H_{11} & H_{12} \\
H_{21} & H_{22}
\end{array}\right)
$$

is the upper left submatrix of matrix $\underline{\hat{H}}$. Please mind that the inverse of $\underline{\underline{H}}, \underline{H}^{-1}$, is not equal to the upper left submatrix of the matrix $\underline{\hat{H}}^{-1}$, but

$$
\underline{\mathrm{H}}^{-1}=\frac{1}{\operatorname{det} \underline{\mathrm{H}}}\left(\begin{array}{cc}
H_{22} & -H_{12} \\
-H_{21} & H_{11}
\end{array}\right),
$$

where the absolute value of the determinant of $\underline{\mathrm{H}}_{s}$ is equal to $\cos \vartheta_{s}$, and $\left|\operatorname{det} \underline{\mathrm{H}}_{g}\right|=\cos \vartheta_{g}$. Introducing $\underline{\mathrm{N}}$ as the upper left $2 \times 2$ submatrix of $\underline{\hat{N}}$, the $2 \times 2$ matrix $\underline{\mathrm{Q}}_{2}^{-1}$ results in

$$
\underline{\mathrm{Q}}_{2}^{-1}=\underline{\mathrm{H}}_{s}^{-\top} \underline{\mathrm{N}}_{g}^{-1}
$$

With $\operatorname{det} \underline{Q}_{2}=1 / \operatorname{det} \underline{Q}_{2}^{-1}$, the spreading in terms of matrix $\underline{\hat{N}}$ can therefore be expressed as

$$
\mathcal{L}=\frac{1}{V_{s}} \sqrt{\left|\frac{\cos \vartheta_{s} \cos \vartheta_{g}}{N_{x x} N_{y y}-N_{x y} N_{y x}}\right|}=\sqrt{\frac{V_{g}}{V_{s}}} \sqrt{\left|\frac{p_{0_{z}} q_{0_{z}}}{N_{x x} N_{y y}-N_{x y} N_{y x}}\right|} .
$$


Other expressions using different matrix elements of $\underline{\hat{N}}$ can be found with the relationships between the matrix elements (5.2.8). Here I give only those depending on the elements of $\underline{\hat{N}}$ that can be determined directly from traveltimes, i.e. not using the $N_{3 i}$ elements (see Section 3.1):

$$
\begin{aligned}
& \mathcal{L}=\sqrt{\frac{V_{g}}{V_{s}}} \sqrt{\mid \frac{p_{0_{z}} q_{0_{y}}}{N_{y x} N_{x z}-N_{x x} N_{y z} \mid}}, \\
& \mathcal{L}=\sqrt{\frac{V_{g}}{V_{s}}} \sqrt{\mid \frac{p_{0_{z}} q_{0_{x}}}{N_{x y} N_{y z}-N_{y y} N_{x z} \mid}} .
\end{aligned}
$$

These results are equivalent to a result given by Hubral et al. (1992a), who show that the geometrical spreading can be expressed by

$$
\mathcal{L}=\frac{1}{V_{s}} \sqrt{\left|\cos \theta_{s} \cos \theta_{g} \operatorname{det} \underline{\mathrm{B}}\right|} .
$$

Here, $\theta_{s}$ is the emergence angle at the anterior surface and $\theta_{g}$ the incidence angle at the posterior surface. The $2 \times 2$ matrix $\underline{B}$ is a submatrix of the ray propagator $\underline{\underline{T}}$ (see Chapter 2 and 4). Using Equation (4.2.8) from Chapter 4 yields

$$
\mathcal{L}=\frac{1}{V_{s}} \sqrt{\left|\frac{\cos \theta_{s} \cos \theta_{g}}{\tilde{N}_{11} \tilde{N}_{22}-\tilde{N}_{12} \tilde{N}_{21}}\right|} .
$$

If the anterior and posterior surfaces coincide with the $x$ - $y$-surface of the Cartesian coordinate system, the angles $\theta_{s}$ and $\theta_{g}$ in Equation (5.2.18) equal the angles $\vartheta_{s}$ and $\vartheta_{g}$. Since in this case the $\tilde{x}$ - $\tilde{y}$-surface follows from the $x$-y-surface by a simple rotation (the determinant of the rotation matrix is equal to 1 ), the determinants of $\underline{N}$ and $\underline{\tilde{N}}$ are equal. Insertion into Equation (5.2.18) leads to

$$
\mathcal{L}=\frac{1}{V_{s}} \sqrt{\left|\frac{\cos \theta_{s} \cos \theta_{g}}{\operatorname{det} \underline{\tilde{N}}}\right|}=\frac{1}{V_{s}} \sqrt{\left|\frac{\cos \vartheta_{s} \cos \vartheta_{g}}{\operatorname{det} \underline{\mathrm{N}}}\right|},
$$

which is the same result as in Equation (5.2.16). Results for other orientations of the anterior and posterior surfaces can be found accordingly.

\subsection{Examples}

To validate the method, this section starts with models whose analytic solution for the spreading and traveltimes is known. The first example is a homogeneous model with $V_{0}=3 \mathrm{~km} / \mathrm{s}$. The input traveltimes were given on a $100 \mathrm{~m}$ coarse grid. Two sets of input traveltimes were applied. The first contains analytic traveltimes. The second set was obtained with an FD eikonal solver using the Vidale (1990) algorithm on a 10m grid and subsequent resampling of the resulting traveltimes. Hyperbolic and parabolic traveltime 
coefficients were determined from the traveltimes. The spreading at the coarse grid points was computed with Equation (5.2.16). Trilinear interpolation was carried out onto a fine $10 \mathrm{~m}$ grid using the spreading values at the coarse grid points. The results were compared to analytically computed geometrical spreading. The residual errors are displayed in Figure 5.4 for both sets of input traveltimes.

The spreading that was computed from the hyperbolic coefficients has smaller errors than that from the parabolic coefficients. This result agrees with the higher errors for the parabolic than for hyperbolic coefficients which was shown in Section 3.3. Also, Figure 5.4 shows that the errors in spreading computed from Vidale traveltimes follow the errors of these traveltimes (see Figure 5.5). The main contributions to the errors from analytic traveltimes come from the trilinear interpolation of the spreading onto the fine grid. Since the errors near the source are the highest, a region of $100 \mathrm{~m}$ depth under the source was excluded from the statistics. Errors near the source are higher because of the high wavefront curvature there. Also, since the geometrical spreading near the source is small, the relative error is rather sensitive there. The median and maximum errors are given in Table 5.1 .

Table 5.1: Relative errors in percent of geometrical spreading for two generic models using analytic and numerical traveltimes as input data. A layer of $100 \mathrm{~m}$ depth under the source was excluded from the statistics.

\begin{tabular}{|lll|rr|}
\hline Model & Traveltimes & Coefficients & $\begin{array}{r}\text { Median of } \\
\text { rel. error [\%] }\end{array}$ & $\begin{array}{r}\text { Maximum } \\
\text { rel. error [\%] }\end{array}$ \\
\hline Homogeneous & analytic & hyperbolic & 0.301 & 14.5 \\
Homogeneous & analytic & parabolic & 1.01 & 61.8 \\
Homogeneous & Vidale & hyperbolic & 0.69 & 14.6 \\
Homogeneous & Vidale & parabolic & 1.55 & 61.4 \\
\hline Gradient & analytic & hyperbolic & 0.251 & 14.1 \\
Gradient & analytic & parabolic & 1.03 & 61.8 \\
Gradient & Vidale & hyperbolic & 0.709 & 14.2 \\
Gradient & Vidale & parabolic & 1.61 & 61.5 \\
\hline
\end{tabular}

A constant velocity gradient model with $V_{0}=3 \mathrm{~km} / \mathrm{s}$ and $\partial V / \partial z=0.5 \mathrm{~s}^{-1}$ with the same dimensions as the homogeneous model is the second example. The resulting error distributions have the same properties as those of the homogeneous model. The errors are displayed in Figure 5.6. The maximum and median errors are also given in Table 5.1.

As an example for a complex velocity model, I have again chosen the Marmousi model (see Figure 3.10). Unlike for the traveltime example in Section 3.4, I have no reference solution to evaluate the resulting geometrical spreading. However, for a 2-D version of the Marmousi model the geometrical (line source) spreading was available for comparison from a wavefront construction implementation by Ettrich and Gajewski (1996). This 


\section{Geometrical Spreading for a Homogeneous Model using Analytic Input Traveltimes:}

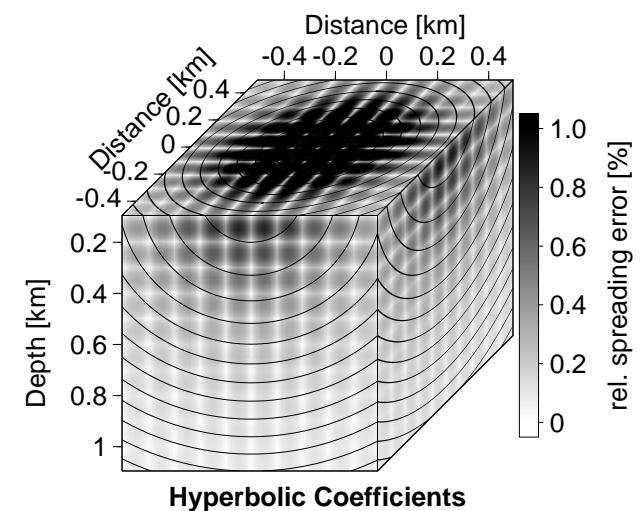

Hyperbolic Coefficients

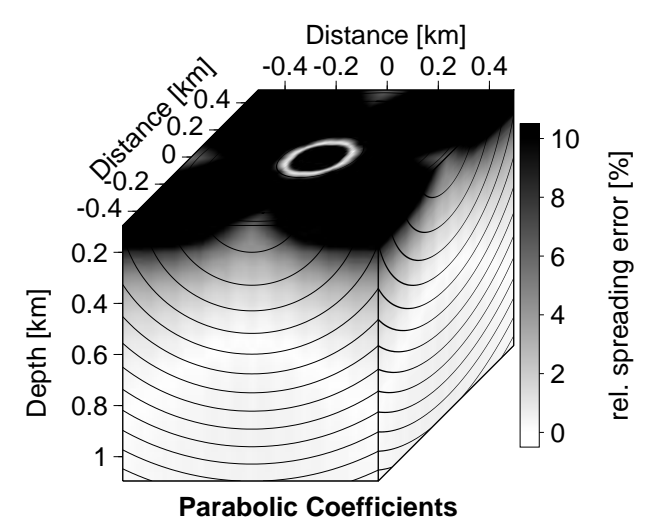

Parabolic Coefficients

using Vidale Input Traveltimes:
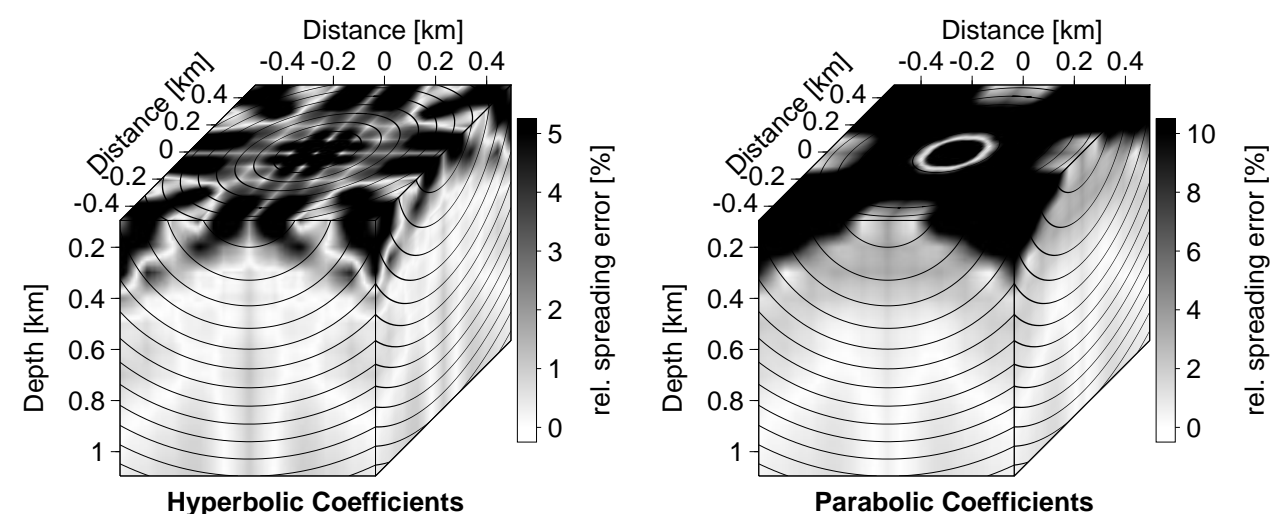

Figure 5.4: Relative errors in geometrical spreading for a homogeneous model, on the right for results from hyperbolic coefficients, left for parabolic coefficients. The top row shows results from analytic input traveltimes, the bottom row those from Vidale traveltimes. The errors of the "parabolic" spreading are larger than those from hyperbolic coefficients. The errors in spreading from Vidale traveltimes follow the traveltime errors of the Vidale algorithm (see Figure 5.5). Please note the different error scales.

program was also used to compute the input traveltimes, where $62.5 \mathrm{~m}$ was chosen as coarse grid spacing. Hyperbolic traveltime coefficients and the geometrical spreading resulting from them were computed at the coarse grid points. The spreading was subsequently interpolated with bilinear interpolation onto a $12.5 \mathrm{~m}$ fine grid. Figure 5.7 shows the difference between the results from wavefront construction and hyperbolic coefficients. The median of the difference between the results from the two methods is below $1.8 \%$. The two solutions coincide well apart from regions with discontinuous wavefronts where triplications occur. As described in Section 3.6, these must be detected and the individual branches must be treated separately, also using later arrivals. This was not implemented and therefore the spreading is not computed correctly in these regions. The difference of both solutions is also very distinct in the lower left corner of 


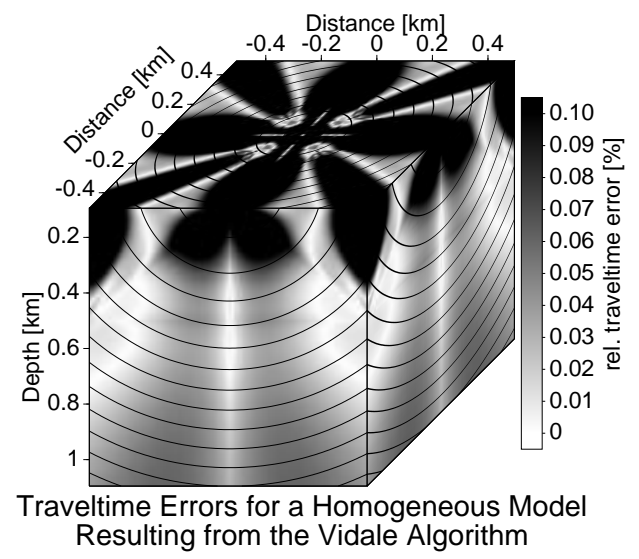

Figure 5.5: Relative errors in percent of Vidale traveltimes for a homogeneous model with $V=3 \mathrm{~km} / \mathrm{s}$. The median error is $0.06 \%$ with a maximum relative error of $0.8 \%$.

the image with a pattern that indicates to follow ray paths. Here I presume that the ray density in the wavefront construction algorithm was too small, leading to a failure in traveltime and spreading interpolation, and thus in the input data.

The geometrical spreading was also computed for the 3-D version of the Marmousi model. Input traveltimes were computed using the Vidale algorithm (cf. Section 3.4) on a 12.5m fine grid, and resampled onto a $125 \mathrm{~m}$ coarse grid. Hyperbolic coefficients and spreading were computed at the coarse grid points. The spreading was then interpolated onto a $12.5 \mathrm{~m}$ fine grid using trilinear interpolation. Figure 5.8 shows the results. As expected, the behaviour of the spreading follows the wavefront curvature indicated by the isochrones. In some regions (e.g., those pointed at by the arrows), a speckled pattern emerges. This is caused by a deficiency in the traveltimes due to the implementation of the Vidale scheme: the isochrones in these regions are "rippled". As a consequence, the traveltimes are not smooth, leading to errors in the determination of the coefficients. 


\section{Geometrical Spreading for a Gradient Model using Analytic Input Traveltimes:}
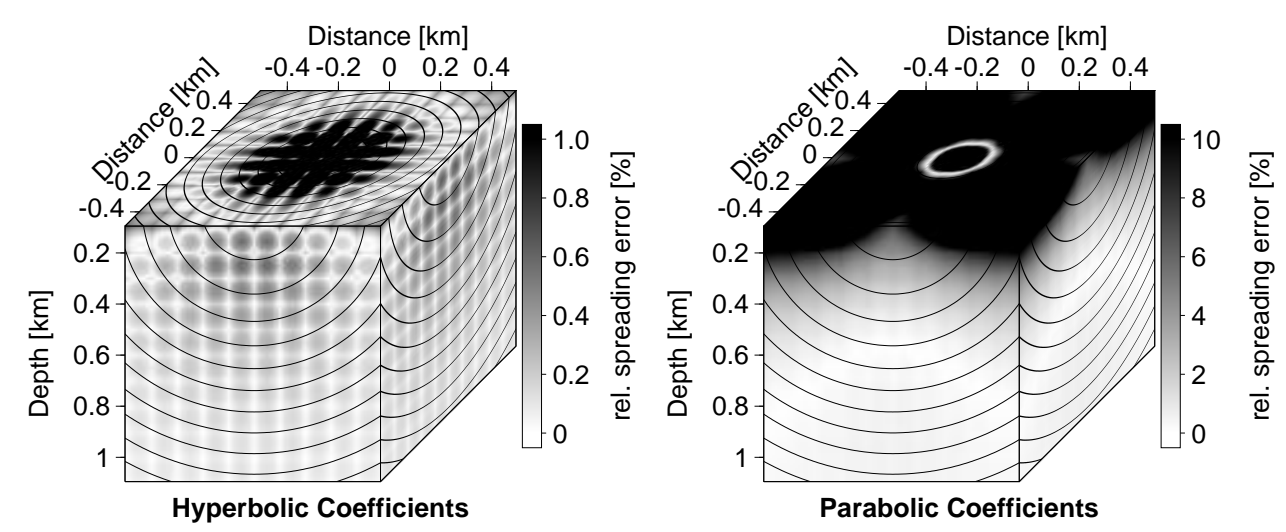

using Vidale Input Traveltimes:
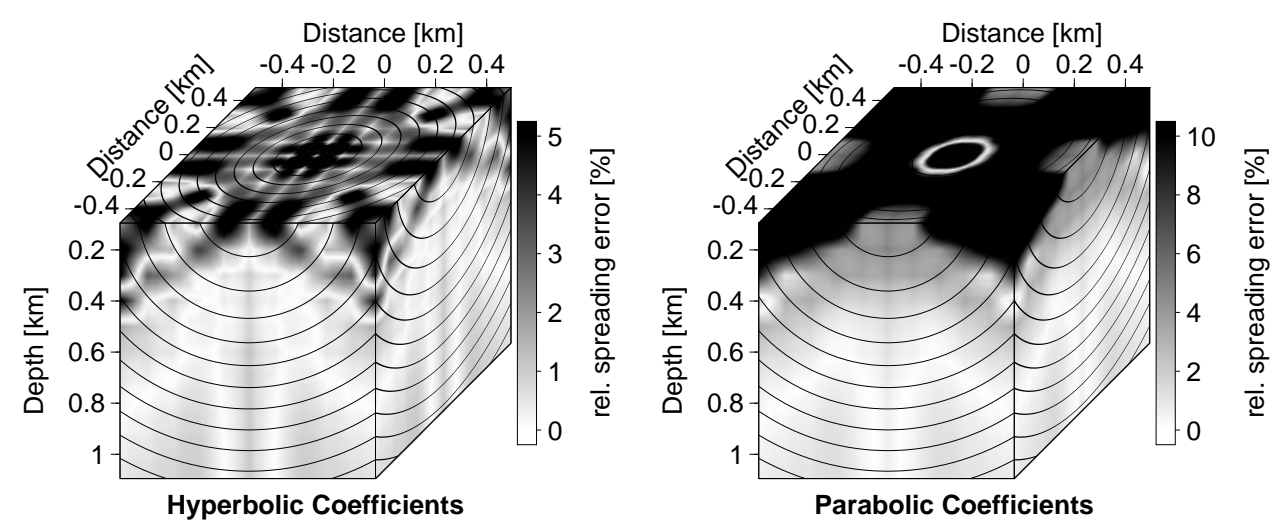

Figure 5.6: Relative errors in geometrical spreading for a gradient model, on the right for results from hyperbolic coefficients, left for parabolic coefficients. The top row shows spreading errors resulting from analytic traveltimes, the bottom row for Vidale traveltimes. Again, the hyperbolic coefficients yield better results than the parabolic ones. Please not the different error scales. 


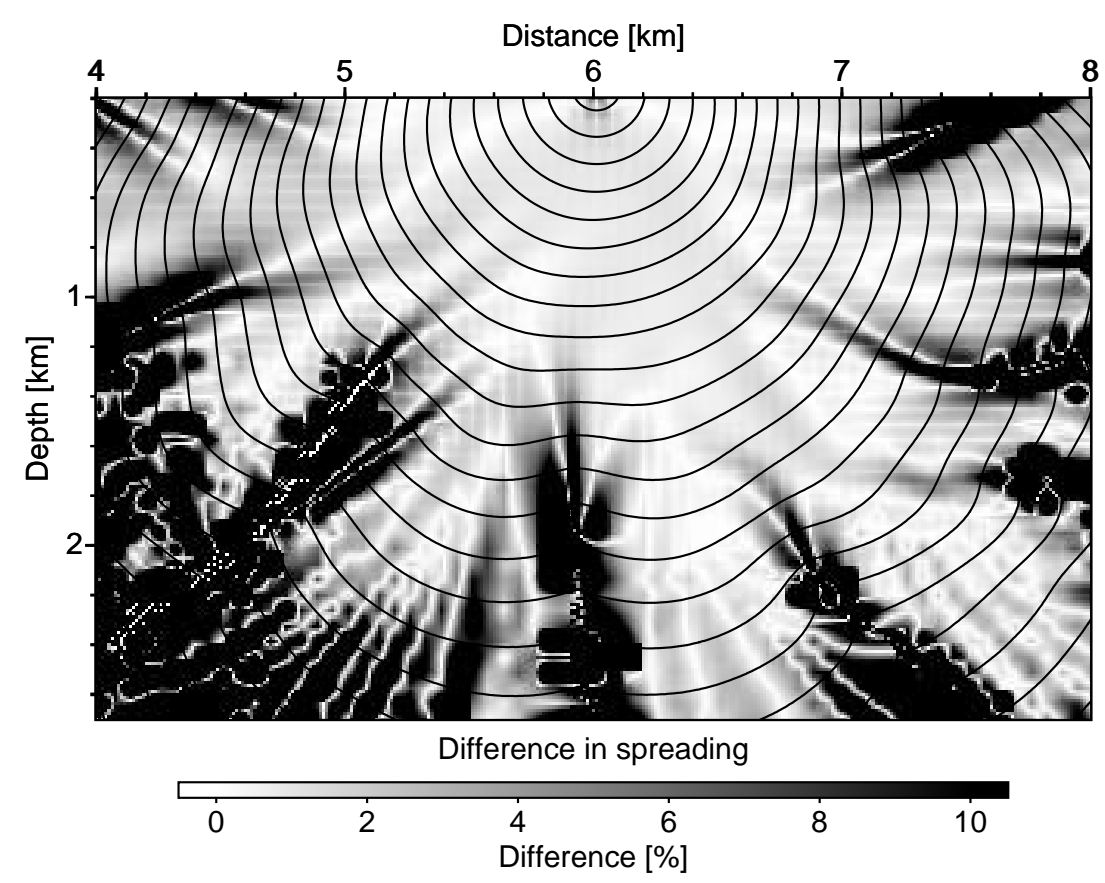

Figure 5.7: Comparison of spreading calculated from hyperbolic coefficients to spreading from a wavefront construction implementation. The difference between both is given in percent. Regions where both results deviate are addressed in the text.

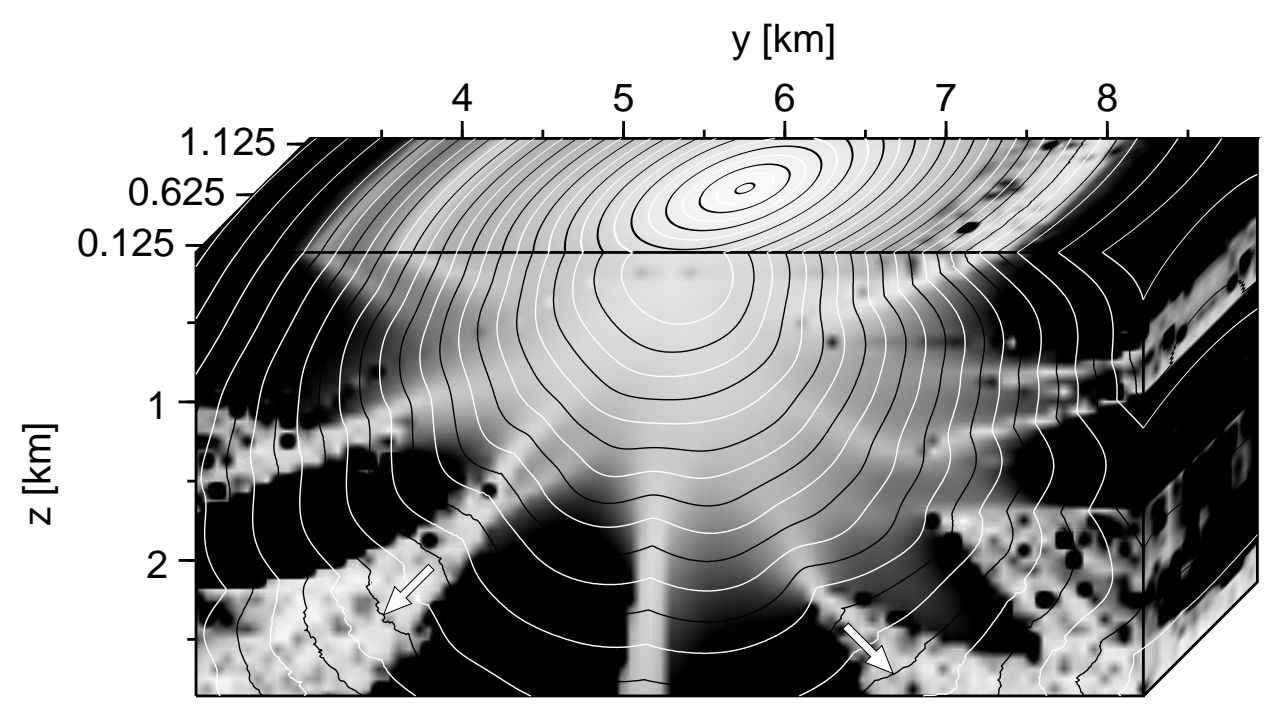

Marmousi model: geometrical spreading

Figure 5.8: Geometrical spreading for the Marmousi model. The arrows indicate two of the regions where the input traveltime data is corrupt, therefore a speckled pattern in the spreading arises (see text). The reason is a deficiency in the FD implementation for the traveltime generation. 


\section{Chapter 6}

\section{Amplitude Preserving Migration}

Migration is an inversion operation that recovers the structure of the subsurface from seismic reflection data. It moves the reflections in the input data to their correct locations and inclinations. Although an initial velocity model must already exist migration provides a better focused image of the subsurface. This is also possible if lateral velocity variations exist, which makes it a powerful tool compared to other imaging techniques, as, e.g., NMO-DMO-stacking. Amplitude preserving migration is a specific type of Kirchhoff migration. In addition to the structural image it also provides information on the reflection strength of the reflectors in the model, leading to estimates of the shear properties of the subsurface. This information is a key feature for reservoir characterisation.

In this chapter I review the existing concept of amplitude preserving migration, also named "true amplitude migration". Three different theoretical approaches have been published during the last 15 years. All of them apply weight functions to the diffraction stack to countermand the loss in amplitude that is caused by geometrical spreading. One method was introduced by Keho and Beydoun (1988), as an extension of the classical Kirchhoff theory (Gardner et al., 1974). It is based on the wave field concepts and gives results for the common shot (CS) and common receiver (CR) configurations. The weight functions were derived using WBKJ ray theoretical Green's function amplitudes. Another approach is that of Bleistein (1987) whose weight functions are based upon Beylkin's determinant (Beylkin, 1985). A work by Červený and deCastro (1993) relates the Beylkin determinant to quantities that can be computed by dynamic ray tracing. This approach can be applied to the common offset (CO) configuration in addition to CS and CR data. The theory behind the third approach was derived by Schleicher et al. (1993a). They propose a weight function that can be derived using paraxial ray theory for arbitrary configuration. A comparison of the three methods can be found in Hanitzsch (1997).

The chapter begins with a short introduction to migration, especially migration of the Kirchhoff type. The next section considers the derivation of a general expression for the weight functions in 3-D media. Since this thesis uses the approach of Schleicher et al. (1993a), I will give a summary of their work. Two quantities are the main ingredients to the weight functions, the geometrical spreading $\mathcal{L}$ and a matrix, $\underline{\mathrm{H}}_{\mathrm{F}}$, which will be 
explained in the following section. Both, $\mathcal{L}$ and $\underline{\mathrm{H}}_{\mathrm{F}}$ can be expressed in terms of second order traveltime derivatives, which leads to the next section on the final weight function. The corresponding weights for migration in 2-D and 2.5-D are also given, although without derivation. This concludes the theoretical part of the chapter. The next two sections are dedicated to application of the method, first to PP data, followed by a PS converted wave example.

\subsection{Introduction to Seismic Migration}

The aim of a seismic reflection experiment is to gain information about the subsurface. This concerns the material properties (densities and elastic parameters) as well as structural information, like geometry and position of reflectors. Although one can quite easily simulate the results from a seismic experiment if the velocities and structural information are given, the inverse process is much more complicated. A zero-offset time section will display an image of the subsurface. This image, however, is distorted: reflectors may not be shown at their correct positions and their inclinations will be wrong. Further confusion is added by diffraction events and later arrivals, e.g., from trough-like structures. Migration is an inversion technique which can reconstruct the true image of the subsurface. The term to migrate has the meaning to move: the reflections which are present in the seismic data are moved to their correct positions and inclinations. At the same time, diffractions are collapsed. Therefore, the migrated image is a focused and corrected image of the subsurface.

Figure 6.1 shows schematically, how migration works. Each subsurface point under consideration is treated as a diffraction point. The diffraction traveltime curve for the point is constructed (how this can be done will be addressed below) and the traces are stacked along that curve. This process is called Kirchhoff migration. Figure 6.1 clearly illustrates that the summation will only be constructive if the point under consideration is on the reflector. Consider now what would happen, if the event in Figure 6.1 were a diffracted event. In this case the stack would only give a non-vanishing result if the subsurface point and the scatterer which causes the diffracted event coincide. This means that a diffraction in the seismic section which is caused by a point scatterer is collapsed into a point at the correct position by the migration. We can think of a reflector as an ensemble of point scatterers. Superposition of the individual points leads to the reconstructed reflector, as shown by Figure 6.2. The resulting image can be either in depth, if the migration output for the image point is written to its position, or in time, where the migration output is written to the apex of the stacking surface. Please note that the terms depth migration and time migration are not defined by either a depth or a time section. They are different concepts, which will be addressed now.

In order to construct the stacking surface, information is required on the velocities. The stacking surface in time migration is given by analytic diffraction traveltimes resulting from RMS velocities (Bancroft, 1998). This is a simplification which does not hold for complex models, especially if strong lateral velocity variations are present. Therefore it 

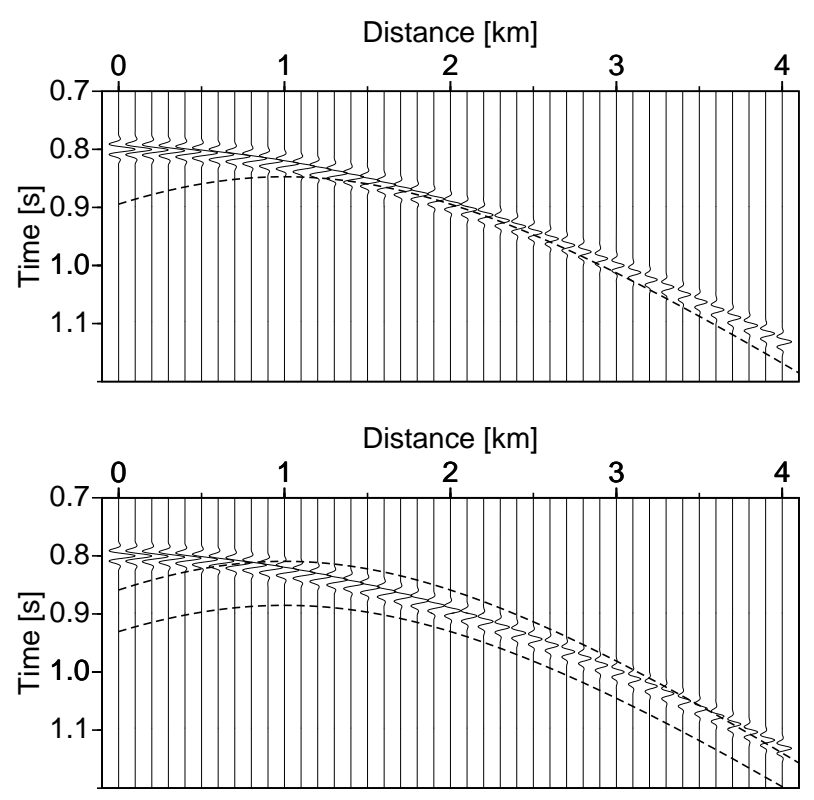

Figure 6.1: Principle of Kirchhoff migration. The dashed lines indicate diffraction traveltime curves. For the top figure the diffraction point lies on the reflector which caused the events in the section. The diffraction traveltime curve is tangent to the reflection. The summation of the traces along the diffraction curve is constructive and the stack result does not vanish. The bottom figure shows two diffraction curves for points that are not on the reflector. In both cases the stack result will be negligible compared to that for the point on the reflector.

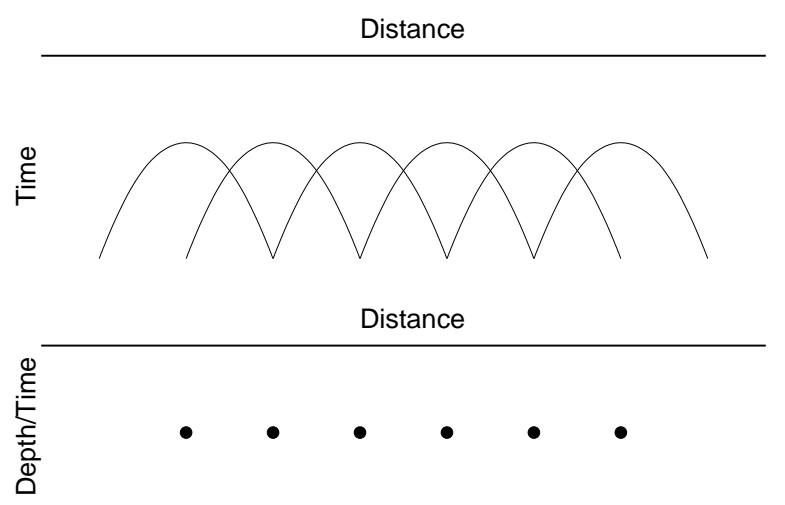

a)

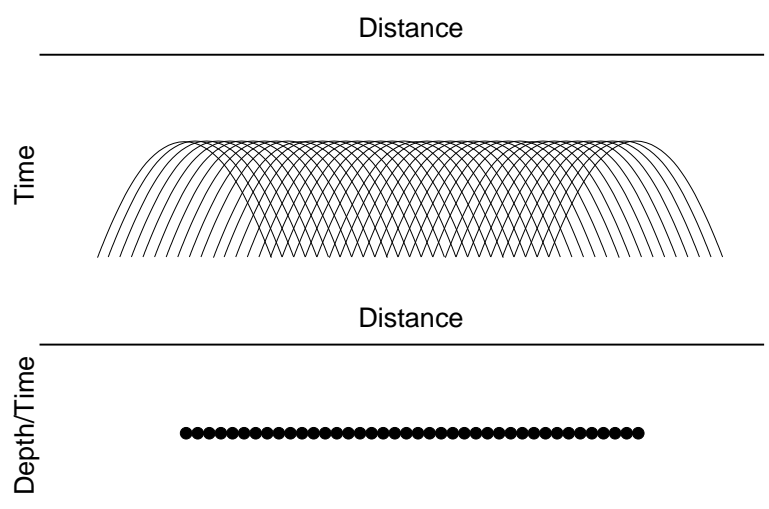

b)

Figure 6.2: Migration as superposition of point scatterers. Figure a) shows diffraction events for individual point scatterers in a schematic seismic section (top). The bottom of a) shows the corresponding migration result, where the diffraction events are collapsed into points. If the spatial sampling of the reflector is dense enough, the shape of the reflector is reconstructed. This is indicated in the lower part of Figure b). 
is better to consider the "real" velocity model to compute diffraction traveltime curves as stacking surfaces, e.g., with ray tracing or FD eikonal solvers. This process is called depth migration. Of course it is much more complicated and time consuming than to carry out a time migration, but complex models can be considered.

Migration requires a velocity model. The reader may wonder why a technique is chosen to construct an image of the subsurface where the information one hopes to obtain must already exist. This is only partially true. Of course it appears to be paradox to use the hoped-for output as input information. But the demands on the input velocity model allow, for example, to use a long wavelength model as an initial velocity model. Such a model can be the result from a tomographic inversion or a simplified model assumed to match the data. The migrated section resulting from such a model will display details not present in the input velocity model. Consider, e.g., a constant velocity input model and and a reflection in the seismic data. The migrated section will show the reflector although it was not present in the constant velocity model. Thus migration yields an image where the structures are more distinct. Also, the better the initial model fits the real one, the better will the focusing of the migrated section be.

Although migration is already a very powerful tool to enhance image quality, there is one aspect which makes it even more important. If suitable weight functions are applied during the stack, the reflection amplitudes can be recovered (provided that the data acquisition and pre-processing has not destroyed amplitude information). This processing is named true-amplitude migration or amplitude-preserving migration. It means, that if, e.g., PP data was recorded, the migration output corresponds to the PP reflection coefficients. Their behaviour in terms of AVO (Amplitude vs. Offset) or AVA (Amplitude vs. Angle) yields information about shear properties because the PP reflection coefficient also depends on the shear properties. This is a very important feature of true-amplitude migration. Because it provides shear information without directly measuring shear waves true-amplitude migration has become a key technique nowadays. Therefore I will explain it in detail in the following section.

\subsection{Derivation of True-Amplitude Weight Functions}

The term true amplitude migration (Bortfeld, 1982) describes primary reflections that are freed of the amplitude loss caused by geometrical spreading. This can be formally expressed by what Schleicher et al. (1993a) define to be a true amplitude trace: they assume an analytic true amplitude signal that can be written as

$$
U_{T A}(t)=\mathcal{L} U\left(\boldsymbol{\xi}, t+\tau_{R}(\boldsymbol{\xi})\right)=\mathcal{R} \mathcal{A} F(t)
$$

In this equation the normalized geometrical spreading is denoted by $\mathcal{L}, \mathcal{A}$ expresses transmission losses, $F(t)$ is the shape of the analytic source pulse, and $\tau_{R}$ is the reflection traveltime. The plane wave reflection coefficient is denoted by $\mathcal{R}$ and $U(\boldsymbol{\xi}, t)$ is the seismic data in terms of the trace coordinates $\boldsymbol{\xi}=\left(\xi_{1}, \xi_{2}\right)$ that describe the source and receiver locations depending on the acquisition geometry (see Appendix B). The seismic data 
$U(\boldsymbol{\xi}, t)$ is assumed to have the form

$$
U(\boldsymbol{\xi}, t)=\frac{\mathcal{R} \mathcal{A}}{\mathcal{L}} F\left(t-\tau_{R}(\boldsymbol{\xi})\right)
$$

Schleicher et al. (1993a) show that if this is the case, a diffraction stack of the form

$$
V(M)=-\left.\frac{1}{2 \pi} \iint_{A} \mathrm{~d} \xi_{1} \mathrm{~d} \xi_{2} W_{3 \mathrm{D}}(\boldsymbol{\xi}, M) \frac{\partial U\left(\xi_{1}, \xi_{2}, t\right)}{\partial t}\right|_{\tau_{D}(\boldsymbol{\xi}, M)}
$$

yields a true amplitude migrated trace obeying (6.2.1) if proper weight functions $W_{3 \mathrm{D}}(\boldsymbol{\xi}, M)$ are applied. In Equation (6.2.3), $V(M)$ is the migration output for an image point $M, A$ is the aperture of the experiment (assumed to provide sufficient illumination), and $\partial U(\boldsymbol{\xi}, t) / \partial t$ is the time derivative of the input seismic trace. This derivative is evaluated at the diffraction traveltime $\tau_{D}(\boldsymbol{\xi}, M)$.

The integral (6.2.3) cannot generally be analytically solved. It can, however be transformed to the frequency domain and for high frequencies be approximately evaluated by the stationary phase method. First, the expression (6.2.2) is inserted into (6.2.3)

$$
V(M)=-\frac{1}{2 \pi} \iint_{A} \mathrm{~d} \xi_{1} \mathrm{~d} \xi_{2} W_{3 \mathrm{D}}(\boldsymbol{\xi}, M) \frac{\mathcal{R} \mathcal{A}}{\mathcal{L}} \frac{\partial F\left(t+\tau_{F}(\boldsymbol{\xi}, M)\right)}{\partial t}
$$

where $\tau_{F}(\boldsymbol{\xi}, M)=\tau_{D}(\boldsymbol{\xi}, M)-\tau_{R}(\boldsymbol{\xi})$ is the difference between diffraction and reflection traveltime. Now (6.2.4) is transformed to the frequency domain where the tilde as in $\tilde{F}(\omega)$ denotes the Fourier transform of the function $F(t)$

$$
\tilde{V}(M)=-\frac{i \omega}{2 \pi} \tilde{F}(\omega) \iint_{A} \mathrm{~d} \xi_{1} \mathrm{~d} \xi_{2} W_{3 \mathrm{D}}\left(\xi_{1}, \xi_{2}, M\right) \frac{\mathcal{R} \mathcal{A}}{\mathcal{L}} \mathrm{e}^{i \omega \tau_{F}\left(\xi_{1}, \xi_{2}, M\right)}
$$

To solve this integral approximately, the stationary phase method can be applied (Bleistein, 1984). It yields a solution to an integral of the form

$$
I(\omega)=\int_{A} \mathrm{~d} \boldsymbol{\xi} f(\boldsymbol{\xi}) \mathrm{e}^{i \omega \tau_{F}(\boldsymbol{\xi})}
$$

for a real valued function $\tau_{F}(\boldsymbol{\xi})$ and sufficiently high frequency $\omega$, provided that there exists a stationary point at $\boldsymbol{\xi}^{*}$ with $\vec{\nabla}_{\xi} \tau_{F}\left(\boldsymbol{\xi}^{*}\right)=0$. For the case of $\operatorname{dim}(\boldsymbol{\xi})=2$ as in (6.2.5), the integral (6.2.6) has the approximate solution

$$
I(\omega) \approx \frac{2 \pi}{\omega} \frac{f\left(\boldsymbol{\xi}^{*}\right)}{\sqrt{\left|\operatorname{det} \underline{\mathrm{H}}_{\mathrm{F}}\right|}} \mathrm{e}^{i \omega \tau_{F}\left(\boldsymbol{\xi}^{*}\right)+i \mathrm{sgn} \underline{\mathrm{H}}_{\mathrm{F}}},
$$

corresponding to the leading term of an asymptotic expansion in $\omega$. The $2 \times 2$ matrix $\underline{\boldsymbol{H}}_{F}$ is given by

$$
\underline{\mathrm{H}}_{F_{I J}}=\frac{\partial^{2} \tau_{F}\left(\boldsymbol{\xi}^{*}\right)}{\partial \xi_{I} \partial \xi_{J}} .
$$


I assume that the stationary point is isolated and unique within the aperture. I also assume that it is not a caustic point, i.e. $\operatorname{det} \underline{H}_{F} \neq 0$. The sign of the matrix $\underline{H}_{F}, \operatorname{sgn} \underline{H}_{F}$, is the number of positive minus the number of negative eigenvalues of $\underline{\mathrm{H}}_{\mathrm{F}}$. Application of the stationary phase method to (6.2.5) corresponds to an expansion of the phase function $\tau_{F}(\boldsymbol{\xi}, M)$ up to second order with respect to the stationary point

$$
\tau_{F}(\boldsymbol{\xi}, M)=\tau_{F}\left(\boldsymbol{\xi}^{*}, M\right)+\left(\boldsymbol{\xi}-\boldsymbol{\xi}^{*}\right)^{\top} \underline{\mathrm{H}}_{\mathrm{F}}\left(\boldsymbol{\xi}-\boldsymbol{\xi}^{*}\right)
$$

and yields the following result

$$
\tilde{V}(M) \approx \tilde{F}(\omega) W_{3 \mathrm{D}}\left(\boldsymbol{\xi}^{*}, M\right) \frac{\mathcal{R} \mathcal{A}}{\mathcal{L} \sqrt{\left|\operatorname{det} \underline{\mathrm{H}}_{\mathrm{F}}\right|}} \mathrm{e}^{i \omega \tau_{F}\left(\boldsymbol{\xi}^{*}, M\right)-\frac{i \pi}{4}\left(2-\mathrm{sgn} \underline{\mathrm{H}}_{\mathrm{F}}\right)} .
$$

If no stationary point exists inside the aperture, the integral will yield only a negligible result of the order $\omega^{-1}$ caused by contributions from the boundaries of the aperture. This can be suppressed by applying a taper (see Schleicher et al. 1993a). The result (6.2.10) transformed back into the time domain reads

$$
V(M) \approx W_{3 \mathrm{D}}\left(\boldsymbol{\xi}^{*}, M\right) \frac{\mathcal{R} \mathcal{A}}{\mathcal{L} \sqrt{\left|\operatorname{det} \underline{\mathrm{H}}_{\mathrm{F}}\right|}} F\left(t+\tau_{F}\left(\boldsymbol{\xi}^{*}, M\right)\right) \mathrm{e}^{-\frac{i \pi}{4}\left(2-\operatorname{sgn}_{\mathrm{F}}\right)} .
$$

If the point $M$ lies on a reflector, the diffraction and reflection traveltime curves are tangent to each other in the stationary point, meaning that $\tau_{F}\left(\boldsymbol{\xi}^{*}, M\right)=0$. This leads to

$$
V(M) \approx W_{3 \mathrm{D}}\left(\boldsymbol{\xi}^{*}, M\right) \frac{\mathcal{R} \mathcal{A}}{\mathcal{L} \sqrt{\left|\operatorname{det} \underline{\mathrm{H}}_{\mathrm{F}}\right|}} F(t) \mathrm{e}^{-\frac{i \pi}{4}\left(2-\operatorname{sgn}_{\mathrm{H}}\right)} .
$$

If we consider a point $M$ that does not lie on the reflector, $\tau_{F}\left(\boldsymbol{\xi}^{*}, M\right)$ will not be zero. Since the source pulse $F(t)$ will be zero outside a small time interval $\tau_{\epsilon}, F\left(t+\tau_{F}\left(\boldsymbol{\xi}^{*}, M\right)\right)$ will become zero if $\left|\tau_{F}\left(\boldsymbol{\xi}^{*}, M\right)\right|>\tau_{\epsilon}$. Therefore, (6.2.11) will yield a non-vanishing result only for points on the reflector (or, more precisely, for points that are within the reflector's Fresnel zone). To get an expression for the weight function, the result (6.2.12) is compared to the expression for the true amplitude trace given by (6.2.1). Since both, $V(M)$ and $\mathrm{U}(\boldsymbol{\xi}, \mathrm{t})$, have significant values only, if $M$ is on a reflector, the comparison leads to the weight function for an image point on a reflector, denoted by $M=R$, where

$$
W_{3 \mathrm{D}}\left(\boldsymbol{\xi}^{*}, R\right)=\mathcal{L} \sqrt{\left|\operatorname{det} \underline{H}_{F}\right|} \mathrm{e}^{i \frac{\pi}{2}\left(1-\frac{\operatorname{sgn} \underline{H}_{F}}{2}\right)} .
$$

In the following sections $I$ will give expressions for the matrix $\underline{H}_{F}$ and the geometrical spreading of the reflected event, $\mathcal{L}$. The final result for the weight function which employs these expressions will be better suited for an implementation than Equation (6.2.13) because both, $\mathcal{L}$, and $\underline{\mathrm{H}}_{\mathrm{F}}$, depend on the curvature of the reflector in the image point. The final weight function will not suffer from this disadvantage. As I will also show, the final weight function will be valid for all subsurface points, regardless of $M$ actually being located at a reflector.

Application of the weight function (6.2.13) in its final form in the stacking process will yield a true-amplitude trace as defined by Equation (6.2.1). This migration output still contains 
transmission losses, $\mathcal{A}$, caused by the overburden. However, according to Hanitzsch (1995) transmission loss due to interfaces is negligible except for interfaces with strong impedance contrast. Therefore, transmission losses must be taken into account for these types of interfaces only. This is, for example, the case if a reflector under a salt structure is considered, because of the strong contrast in acoustic impedance between the salt and the surrounding medium. This problem can be dealt with by using layer stripping methods (Hanitzsch, 1995).

\subsection{The Matrix $\underline{H}_{F}$}

Schleicher et al. (1993a) show how the matrix $\underline{H}_{F}$ can be expressed in terms of second order derivative matrices of the traveltimes. They split the traveltime from a source located at a position $\boldsymbol{s}$ to a receiver at $\boldsymbol{g}$ into the two corresponding branches, from $\boldsymbol{s}$ to a subsurface point $M$ at the position $\overline{\boldsymbol{r}}$, and from $\overline{\boldsymbol{r}}$ to $\boldsymbol{g}$, and connect the two branches with the Bortfeld propagator (see Section 2.9). Here, I follow the lines of derivation proposed in Schleicher (1993). I also make use of the fact that the traveltime $\tau(\overline{\boldsymbol{r}}, \boldsymbol{g})$ is equal to $\tau(\boldsymbol{g}, \overline{\boldsymbol{r}})$. The vectors $\boldsymbol{s}$ and $\boldsymbol{g}$ lie in the registration surface whose base vectors are assumed to coincide with the global Cartesian coordinate system introduced in Chapter 3. Therefore they have dimension two, which is distinguished from a 3-D vector carrying a hat . Vector $\overline{\boldsymbol{r}}$ will be used to describe the reflection point. This makes sense only if $\overline{\boldsymbol{r}}$ lies in the reflector surface, therefore $\overline{\boldsymbol{r}}$ is a 2 -D vector in a specific coordinate system associated with the reflector surface. This coordinate system is denoted with a bar ${ }^{-}$. Both $\tau(\boldsymbol{g}, \overline{\boldsymbol{r}})$ and $\tau(\boldsymbol{s}, \overline{\boldsymbol{r}})$ can be expressed in terms of a 2-D variant of the parabolic traveltime expansion Equation (3.1.4) with

$$
\tau(\boldsymbol{s}, \overline{\boldsymbol{r}})=\tau_{1}-\boldsymbol{p}_{0_{1}}^{\top} \Delta \boldsymbol{s}+\overline{\boldsymbol{q}}_{0_{1}}^{\top} \Delta \overline{\boldsymbol{r}}-\Delta \boldsymbol{s}^{\top} \underline{\underline{N}}_{1} \Delta \overline{\boldsymbol{r}}-\frac{1}{2} \Delta \boldsymbol{s}^{\top} \underline{\mathrm{S}}_{1} \Delta \boldsymbol{s}+\frac{1}{2} \Delta \overline{\boldsymbol{r}}^{\top} \underline{\mathrm{G}}_{1} \Delta \overline{\boldsymbol{r}}
$$

and

$$
\tau(\boldsymbol{g}, \overline{\boldsymbol{r}})=\tau_{2}-\boldsymbol{p}_{0_{2}}^{\top} \Delta \boldsymbol{g}+\overline{\boldsymbol{q}}_{0_{2}}^{\top} \Delta \overline{\boldsymbol{r}}-\Delta \boldsymbol{g}^{\top} \underline{\mathrm{N}}_{2} \Delta \overline{\boldsymbol{r}}-\frac{1}{2} \Delta \boldsymbol{g}^{\top} \underline{\mathrm{S}}_{2} \Delta \boldsymbol{g}+\frac{1}{2} \Delta \overline{\boldsymbol{r}}^{\top} \underline{\mathrm{G}}_{2} \Delta \overline{\boldsymbol{r}}
$$

The sums of Equations (6.3.14) and (6.3.15) yield $\tau_{D}$ and $\tau_{R}$. For the diffraction traveltime, the diffractor position is fixed at $\overline{\boldsymbol{r}}_{0}$, and thus with $\Delta \overline{\boldsymbol{r}}=0$

$$
\tau_{D}(\boldsymbol{s}, \boldsymbol{g})=\tau_{0}-\boldsymbol{p}_{0}^{\top} \Delta \boldsymbol{s}+\boldsymbol{q}_{0}^{\top} \Delta \boldsymbol{g}-\frac{1}{2} \Delta \boldsymbol{s}^{\top} \underline{\mathrm{S}}_{1} \Delta \boldsymbol{s}-\frac{1}{2} \Delta \boldsymbol{g}^{\top} \underline{\mathrm{S}}_{2} \Delta \boldsymbol{g}
$$

where I have used the abbreviations $\tau_{0}=\tau_{1}+\tau_{2}, \boldsymbol{p}_{0}=\boldsymbol{p}_{0_{1}}$, and $\boldsymbol{q}_{0}=-\boldsymbol{p}_{0_{2}}$. For the reflection traveltime it must be taken into account that variation of source and/or receiver positions will result in a different reflection point $\overline{\boldsymbol{r}}$. Aiming for an expression containing $\Delta \boldsymbol{s}$ and $\Delta \boldsymbol{g}$ only, Snell's law is applied, stating that $\overline{\boldsymbol{q}}_{0_{1}}+\overline{\boldsymbol{q}}_{0_{2}}=\boldsymbol{\nabla}_{r}^{*} \tau_{R}=\mathbf{0}$. This can be solved for $\overline{\boldsymbol{r}}$, and $\overline{\boldsymbol{r}}$ is then eliminated from the sum of Equations (6.3.14) and (6.3.15), resulting in

$$
\tau_{R}(\boldsymbol{s}, \boldsymbol{g})=\tau_{0}-\boldsymbol{p}_{0}^{\top} \Delta \boldsymbol{s}+\boldsymbol{q}_{0}^{\top} \Delta \boldsymbol{g}-\frac{1}{2} \Delta \boldsymbol{s}^{\top} \underline{\underline{S}} \Delta \boldsymbol{s}+\frac{1}{2} \Delta \boldsymbol{g}^{\top} \underline{\overline{\mathrm{G}}} \Delta \boldsymbol{g}-\Delta \boldsymbol{s}^{\top} \underline{\overline{\mathrm{N}}} \Delta \boldsymbol{g}
$$


where the matrices $\underline{\bar{N}}, \underline{S}$, and $\underline{\underline{G}}$ are given by

$$
\begin{aligned}
& \underline{\mathrm{S}}=\underline{\mathrm{S}}_{1}+\overline{\mathrm{N}}_{1}\left(\overline{\mathrm{G}}_{1}+\overline{\mathrm{G}}_{2}\right)^{-1} \overline{\mathrm{N}}_{1}^{\top} \\
& \underline{\overline{\mathrm{G}}}=-\underline{\mathrm{S}}_{2}-\underline{\mathrm{N}}_{2}\left(\overline{\mathrm{G}}_{1}+\overline{\mathrm{G}}_{2}\right)^{-1} \underline{\mathrm{N}}_{2}^{\top} \\
& \underline{\overline{\mathrm{N}}}=\underline{\mathrm{N}}_{1}\left(\underline{\mathrm{G}}_{1}+\underline{\mathrm{G}}_{2}\right)^{-1} \underline{\mathrm{N}}_{2}^{\top}
\end{aligned}
$$

A similar result was already derived from application of the ray propagator formalism in Chapter 4. For further simplification, the matrix $\underline{\mathrm{H}}$ is introduced as an abbreviation for

$$
\underline{\mathrm{H}}=\overline{\mathrm{G}}_{1}+\underline{\mathrm{G}}_{2} \text {. }
$$

In the following step, the difference $\tau_{F}$ between Equations (6.3.16) and (6.3.17) is built and at the same time $\Delta \boldsymbol{s}=\boldsymbol{s}-\boldsymbol{s}_{0}$ and $\Delta \boldsymbol{g}=\boldsymbol{g}-\boldsymbol{g}_{0}$ are expressed in trace coordinates $\boldsymbol{\xi}$ using the configuration matrices $\underline{\Sigma}$ and $\underline{\Gamma}$ (see Appendix B). The resulting expression for $\tau_{F}(\boldsymbol{\xi}, M)$ is

$$
\begin{aligned}
\tau_{F}(\boldsymbol{\xi}, M) & = \\
& \frac{1}{2}\left(\boldsymbol{\xi}-\boldsymbol{\xi}^{*}\right)^{\top}\left[\underline{\Sigma}^{\top} \underline{\bar{N}}_{1} \underline{\mathrm{H}}^{-1} \underline{\overline{\mathbf{N}}}_{1}^{\top} \underline{\Sigma}+\underline{\Gamma}^{\top} \underline{\bar{N}}_{2} \underline{\mathrm{H}}^{-1} \underline{\mathbf{N}}_{2}^{\top} \underline{\Gamma}+2 \underline{\Sigma}^{\top} \underline{\bar{N}}_{1} \underline{\mathrm{H}}^{-1} \underline{\mathbf{N}}_{2}^{\top} \underline{\Gamma}\right]\left(\boldsymbol{\xi}-\boldsymbol{\xi}^{*}\right) .
\end{aligned}
$$

Differentiation with respect to $\boldsymbol{\xi}$ yields $\underline{\mathrm{H}}_{\mathrm{F}}$

$$
\underline{\mathrm{H}}_{\mathrm{F}}=\left(\underline{\Sigma}^{\top} \underline{\overline{\mathrm{N}}}_{1}+\underline{\Gamma}^{\top} \underline{\overline{\mathrm{N}}}_{2}\right) \underline{\mathrm{H}}^{-1}\left(\underline{\overline{\mathrm{N}}}_{1}^{\top} \underline{\Sigma}+\underline{\overline{\mathrm{N}}}_{2}^{\top} \underline{\Gamma}\right) \text {. }
$$

This leads to the determinant of $\underline{\mathrm{H}}_{\mathrm{F}}$

$$
\left|\operatorname{det} \underline{H}_{\mathrm{F}}\right|=\frac{\left|\operatorname{det}\left(\underline{\overline{\mathrm{N}}}_{1}^{\top} \underline{\Sigma}+\underline{\overline{\mathrm{N}}}_{2}^{\top} \underline{\Gamma}\right)\right|^{2}}{|\operatorname{det} \underline{\mathrm{H}}|} .
$$

Furthermore, $\operatorname{sgn} \underline{H}_{F}$ equals sgn$\underline{H}$, since $\underline{H}$ is a symmetric matrix.

\subsection{Geometrical Spreading and Final Weight Func- tion}

I have shown in Chapter 5 how the geometrical spreading is related to the matrices $\underline{\hat{N}}$ and $\underline{\mathrm{N}}$. These relationships also apply to the geometrical spreading of a reflected event. With Equation (6.3.18) the spreading can be expressed in terms of the matrices $\underline{\bar{N}}_{I}$ and $\underline{\mathrm{G}}_{I}$, or $\underline{\mathrm{H}}$, following from the decomposition of traveltimes, and resulting in

$$
\begin{aligned}
\mathcal{L} & =\frac{1}{V_{s}} \sqrt{\frac{\cos \vartheta_{s} \cos \vartheta_{g}}{|\operatorname{det} \underline{\bar{N}}|}} \mathrm{e}^{-i \frac{\pi}{2} \kappa} \\
& =\frac{1}{V_{s}} \sqrt{\cos \vartheta_{s} \cos \vartheta_{g}} \sqrt{\frac{|\operatorname{det} \underline{\mathrm{H}}|}{\left|\operatorname{det} \underline{\bar{N}}_{1}\right|\left|\operatorname{det} \underline{\mathrm{N}}_{2}\right|}} \mathrm{e}^{-i \frac{\pi}{2} \kappa} .
\end{aligned}
$$


The KMAH index $\kappa$ of the reflected ray can be decomposed into the KMAH indices of the ray branches $\kappa_{I}$ (Schleicher et al., 1993a)

$$
\kappa=\kappa_{1}+\kappa_{2}+\left(1-\frac{\operatorname{sgn} \underline{\mathrm{H}}}{2}\right) .
$$

The KMAH indices $\kappa_{I}$ are known if a suitable traveltime generator is used, as, e.g., the method by Coman and Gajewski (2001), which outputs later-arrival traveltimes sorted for their KMAH index. The angles $\vartheta_{s}$ and $\vartheta_{g}$ are the emergence angle at the source and the incidence angle at the receiver. They can be computed from the slowness vectors at the source and receiver, i.e.,

$$
\begin{aligned}
& \cos \vartheta_{s}=\sqrt{1-V_{s}^{2} \boldsymbol{p}_{0_{1}} \cdot \boldsymbol{p}_{0_{1}}} \\
& \cos \vartheta_{g}=\sqrt{1-V_{g}^{2} \boldsymbol{p}_{0_{2}} \cdot \boldsymbol{p}_{0_{2}}}
\end{aligned}
$$

Equation (6.2.13) together with (6.3.22), (6.4.24), and (6.4.25) yields the final expression for the weight function:

$$
W_{3 \mathrm{D}}\left(\boldsymbol{\xi}^{*}, M\right)=\frac{1}{V_{s}} \sqrt{\cos \vartheta_{s} \cos \vartheta_{g}} \frac{\left|\operatorname{det}\left(\underline{\overline{\mathrm{N}}}_{1}^{\top} \underline{\Sigma}+\underline{\overline{\mathrm{N}}}_{2}^{\top} \underline{\Gamma}\right)\right|}{\sqrt{\left|\operatorname{det} \underline{\bar{N}}_{1}\right|\left|\operatorname{det} \underline{\bar{N}}_{2}\right|}} \mathrm{e}^{-i \frac{\pi}{2}\left(\kappa_{1}+\kappa_{2}\right)}
$$

Equation (6.4.27) contains only quantities which do not depend on whether the image point $M$ is a reflection point or not. Therefore, Equation (6.4.27) can be applied for all subsurface points. Since the matrices $\underline{G}_{I}$ do not occur anymore, Equation (6.4.27) is furthermore independent of the reflector curvature. For the determination of the matrices $\underline{\bar{N}}_{I}$ from $\underline{\hat{N}}_{I}$, however, the reflector inclination is required. I assume that this information is available as a priori information, e.g., from a previous migration or the velocity model itself. For image points which do not lie on a reflector, the inclination will not be available. Since the migration output for these points will be negligible, I set the inclination angle to zero at these points. The problem of dealing with the required $a$ priori reflector information will be addressed in more detail in Chapter 7 .

Shortly after the original thesis was completed I have developed an alternative formulation for the weight functions that does not require a priori information any longer. A visual inspection of the migration results for the examples presented below showed no differences. The new, reflector-independent weight functions are derived in Appendix E.

All quantities in Equation (6.4.27) can be determined from traveltime tables. The matrices $\underline{\bar{N}}_{I}$ are computed from the $\underline{\hat{\mathrm{N}}}_{I}$ which are also needed for the diffraction time surface, as are the slownesses that lead to the angles $\vartheta_{I}$. Since all coefficients must be known for the computation of the diffraction time surface anyway, the determination of the weight functions takes only a small part of the computational time required for the migration. I name this approach traveltime-based true-amplitude migration because coarsely gridded traveltime tables are the only input required. Since very small additional effort must be spent for the weights, it is very efficient. The high accuracy of the coefficients has 
already been stated in Chapter 3. The accuracy of the geometrical spreading computed from the coefficients in Chapter 5 supports the expected high accuracy of the recovered reflection coefficients. Before I will demonstrate the method with applications, however, I will derive corresponding weight functions for 2-D media and situations, where a 2.5-D geometry is considered.

\subsection{Weight Functions for 2.5-D and 2-D}

Sometimes seismic data is only available for sources and receivers constrained to a single straight acquisition line. Processing of this data with techniques based on 2-D wave propagation does not yield satisfactory results because the (spherical) geometrical spreading in the data caused by the 3 -D earth does not agree with the cylindrical (i.e. line source) spreading implied by the $2-\mathrm{D}$ wave equation. The problem can be dealt with by assuming the subsurface to be invariant in the off-line direction. This symmetry is called to be 2.5-dimensional (Bleistein, 1986). Apart from the geometrical spreading, the properties involved do not depend on the out-of-plane variable and can be computed with 2-D techniques. The geometrical spreading can be split into an in-plane part that is equal to the 2-D spreading and an out-of-plane contribution. For the described symmetry, the product of both equals the spreading following from the 3 -D wave equation.

Equation (6.2.13) is an expression for a weight function if the diffraction stack is carried out over the aperture in $\xi_{1}$ and $\xi_{2}$. Since in the 2.5-D case the data comes from a single acquisition line (assumed to coincide with the $\xi_{1}$ coordinate), the stack is performed only over $\xi_{1}$. In this case the input data is $U\left(\xi_{1}, \xi_{2}, t\right)=U\left(\xi_{1}, \xi_{2}^{*}, t\right)$ where the asterisk denotes the stationary point (which is in this case the $\xi_{2}$ position of the source-receiver line). The weight functions for 2.5-D symmetry can be derived following the same lines as for 3-D. Equation (6.2.3) becomes

$$
V(M)=-\left.\frac{1}{2 \pi} \int_{A} \mathrm{~d} \xi_{1} \int_{-\infty}^{\infty} \mathrm{d} \xi_{2} W_{3 \mathrm{D}}\left(\xi_{1}, \xi_{2}, M\right) \frac{\mathcal{R} A}{\mathcal{L}} \frac{\partial F(t)}{\partial t}\right|_{\tau_{F}\left(\xi_{1}, \xi_{2}, M\right)}
$$

After carrying out the integration over $\xi_{2}$ in the frequency domain by applying stationary phase method (following Martins et al. 1997) $V(M)$ becomes

$$
\begin{aligned}
V(M) & =\left.\frac{1}{\sqrt{2 \pi}} \int_{A} \mathrm{~d} \xi_{1} W_{3 \mathrm{D}}\left(\xi_{1}, \xi_{2}^{*}, M\right)\left(\left.\frac{\partial^{2} \tau_{D}}{\partial \xi_{2}^{2}}\right|_{\xi_{2}^{*}}\right)^{-\frac{1}{2}} \mathrm{e}^{-i \frac{\pi}{4}} \frac{\mathcal{R} A}{\mathcal{L}} f[F(t)]\right|_{\tau_{F}\left(\xi_{1}, \xi_{2}^{*}, M\right)} \\
& =\frac{1}{\sqrt{2 \pi}} \int_{A} \mathrm{~d} \xi_{1} W_{3 \mathrm{D}}\left(\xi_{1}, \xi_{2}^{*}, M\right)\left(\left.\frac{\partial^{2} \tau_{D}}{\partial \xi_{2}^{2}}\right|_{\xi_{2}^{*}}\right)^{-\frac{1}{2}} \mathrm{e}^{-i \frac{\pi}{4}} f\left[U\left(\xi_{1}, \xi_{2}^{*}, t+\tau_{D}\left(\xi_{1}, \xi_{2}^{*}, M\right)\right)\right] \\
& =\frac{1}{\sqrt{2 \pi}} \int_{A} \mathrm{~d} \xi_{1} W_{2.5 \mathrm{D}}\left(\xi_{1}, \xi_{2}^{*}, M\right) f\left[U\left(\xi_{1}, \xi_{2}^{*}, t+\tau_{D}\left(\xi_{1}, \xi_{2}^{*}, M\right)\right)\right]
\end{aligned}
$$


Note, that in this case not the time derivative of the seismic trace is taken, but a $\sqrt{i \omega}$ filter operation in the frequency domain (commonly called half derivative) is applied instead. This is denoted by the function $f[U(t)]$ in (6.5.29). The 2.5-D weight function is related to the 3 -D weight by

$$
W_{2.5 \mathrm{D}}\left(\xi_{1}, \xi_{2}^{*}, M\right)=W_{3 \mathrm{D}}\left(\xi_{1}, \xi_{2}^{*}, M\right)\left(\left.\frac{\partial^{2} \tau_{D}}{\partial \xi_{2}^{2}}\right|_{\xi_{2}^{*}}\right)^{-\frac{1}{2}} \mathrm{e}^{-i \frac{\pi}{4}}
$$

For the 2.5-D geometry simplifications apply to the matrix $\underline{\mathrm{H}}_{\mathrm{F}}$ and to the spreading, and therefore to the weight function. Let the out-of-plane direction (index 2) coincide with the $y$-axis of the Cartesian system defined by the traveltime tables with $y_{0}=s_{20}=g_{20}=r_{20}$ as $y$-position of the sources and receivers. Then it follows that

$$
\left.N_{22}\right|_{y_{0}}=\left.\hat{N}_{y y}\right|_{y_{0}},\left.\quad G_{22}\right|_{y_{0}}=\left.\hat{G}_{y y}\right|_{y_{0}} \quad \text { and }\left.\quad S_{22}\right|_{y_{0}}=\left.\hat{S}_{y y}\right|_{y_{0}}
$$

From the symmetry one can see that the $y$-components of the slownesses vanish at $y_{0}$ along the profile:

$$
\left.\frac{\partial \tau}{\partial y_{s}}\right|_{y_{0}}=\left.\frac{\partial \tau}{\partial y_{g}}\right|_{y_{0}}=\left.\frac{\partial \tau}{\partial y_{r}}\right|_{y_{0}}=0 \quad \text {. }
$$

From this follows that the matrices $\underline{S}, \underline{G}$ and $\underline{\bar{N}}$ consist only of diagonal elements. Furthermore, the absolute values of the $y y$ - or 22-components of all of the matrices are equal,

$$
\left.N_{22}\right|_{y_{0}}=\left.G_{22}\right|_{y_{0}}=-\left.S_{22}\right|_{y_{0}}
$$

and the sign of $\left.N_{22}\right|_{y_{0}}$ is positive; i.e., $\operatorname{sgn}\left(\left.N_{22}\right|_{y_{0}}\right)=+1$.

As already indicated, the geometrical spreading can be written as the product of an inplane and an out-of-plane contribution. The reason is that the matrix $\underline{\bar{N}}$ consists only of the diagonal elements. Equation (6.4.23) becomes

$$
\mathcal{L}_{2.5 \mathrm{D}}=\frac{1}{V_{s}} \frac{\sqrt{\cos \vartheta_{s} \cos \vartheta_{g}}}{\sqrt{\left|\bar{N}_{11}\right|}} \sqrt{\frac{1}{\left|N_{22}\right|}} \mathrm{e}^{-i \frac{\pi}{2} \kappa}=\sqrt{\sigma} \mathcal{L}_{2 \mathrm{D}}
$$

where $\sqrt{\sigma}=\sqrt{N_{22}^{-1}}$ is the out-of-plane spreading. The in-plane contribution equals the (line source) spreading for $2-\mathrm{D}$ wave propagation given by

$$
\mathcal{L}_{2 \mathrm{D}}=\frac{1}{V_{s}} \frac{\sqrt{\cos \vartheta_{s} \cos \vartheta_{g}}}{|\bar{N}|} \mathrm{e}^{-i \frac{\pi}{2} \kappa}
$$

where the matrix $\underline{\bar{N}}$ reduces to the scalar $\bar{N}\left(=\bar{N}_{11}\right)$. The second-order derivative of the diffraction traveltime with respect to the $\xi_{2}$ component can be determined from Equation (6.3.16). It is

$$
\left.\frac{\partial^{2} \tau_{D}}{\partial \xi_{2}^{2}}\right|_{\xi_{2}^{*}}=-S_{1_{22}}-S_{2_{22}}
$$


This leads to the final weight function for a 2.5-D symmetry

$$
W_{2.5 \mathrm{D}}\left(\xi_{1}, \xi_{2}^{*}, M\right)=\frac{\sqrt{\cos \vartheta_{s} \cos \vartheta_{g}}}{V_{s}} \frac{\left|\bar{N}_{1_{11}} \Sigma_{11}+\bar{N}_{2_{11}} \Gamma_{11}\right|}{\sqrt{\left|\bar{N}_{1_{11}} \bar{N}_{2_{11}}\right|}} \sqrt{\frac{N_{1_{22}}+N_{2_{22}}}{N_{1_{22}} N_{2_{22}}}} \mathrm{e}^{-i \frac{\pi}{2}\left(\kappa_{1}+\kappa_{2}\right)-i \frac{\pi}{4}}
$$

If a 2-D medium with 2-D geometrical spreading (line source) is assumed, the stack (6.5.29) can be used with the 2-D weight function

$$
W_{2 \mathrm{D}}(\xi, M)=\mathcal{L} \sqrt{H_{F}} \mathrm{e}^{i \frac{\pi}{2}\left(1-\frac{\operatorname{sgn} H_{F}}{2}\right)}
$$

In this case, the matrix $\underline{\mathrm{H}}_{\mathrm{F}}$ is reduced to a scalar $H_{F}$. The final weight function for 2-D reads

$$
W_{2 \mathrm{D}}(\xi, M)=\frac{\sqrt{\cos \vartheta_{s} \cos \vartheta_{g}}}{V_{s}} \frac{\left|\bar{N}_{1} \Sigma+\bar{N}_{2} \Gamma\right|}{\sqrt{\left|\bar{N}_{1} \bar{N}_{2}\right|}} \mathrm{e}^{-i \frac{\pi}{2}\left(\kappa_{1}+\kappa_{2}\right)-i \frac{\pi}{4}}
$$

This 2-D weight function is equivalent to the one given by Hanitzsch et al. (1994).

\subsection{Application to PP Data}

In this section I will apply the new method to two velocity models. Simple examples were chosen in order to allow for comparison of numerically and analytically computed amplitudes. The method is, however, not limited to homogeneous velocity layer models. For convenience reasons the examples are restricted to 2.5-D. For both examples the velocity model was only used to compute the traveltimes with a finite difference eikonal solver (FDES, Vidale 1990) using an implementation of Leidenfrost (1998). These traveltimes were resampled from the original 10m fine grid required by FDES for sufficient accuracy and stored on a coarse grid of $50 \mathrm{~m}$ in either direction. Diffraction traveltimes were interpolated from this coarse grid onto a fine migration grid of $5 \mathrm{~m}$ in $z$-direction using the hyperbolic traveltime approximation (3.2.2). The migration weights were also computed from the coarse gridded traveltimes using the coefficients determined from the hyperbolic approximation, see Chapter 3 .

The first model has a planar horizontal reflector at a depth of $2500 \mathrm{~m}$. The $\mathrm{P}$ velocity is $V_{P_{1}}=5 \mathrm{~km} / \mathrm{s}$ in the upper part of the model and $V_{P_{2}}=6 \mathrm{~km} / \mathrm{s}$ below the reflector. The $\mathrm{S}$ velocities $V_{S_{i}}=V_{P_{i}} / \sqrt{3}$ were used for reflection coefficients; only PP reflections were considered. The density is $\rho\left[\mathrm{g} / \mathrm{cm}^{3}\right]=1.7+0.2 V_{P}[\mathrm{~km} / \mathrm{s}]$. Ray synthetic seismograms were computed using the SEIS88 package (Červený and Pšenčík, 1984) for a receiver line consisting of 100 equidistantly positioned receivers with a spacing of $50 \mathrm{~m}$ and the first receiver $50 \mathrm{~m}$ away from the point source. The resulting common shot section is shown in Figure 6.3.

Figure 6.4 shows the relevant part of the resulting migrated depth section for this one shot together with the model. The reflector was migrated to the correct position and the source pulse, a Gabor wavelet, was reconstructed. Since there are no transmission 


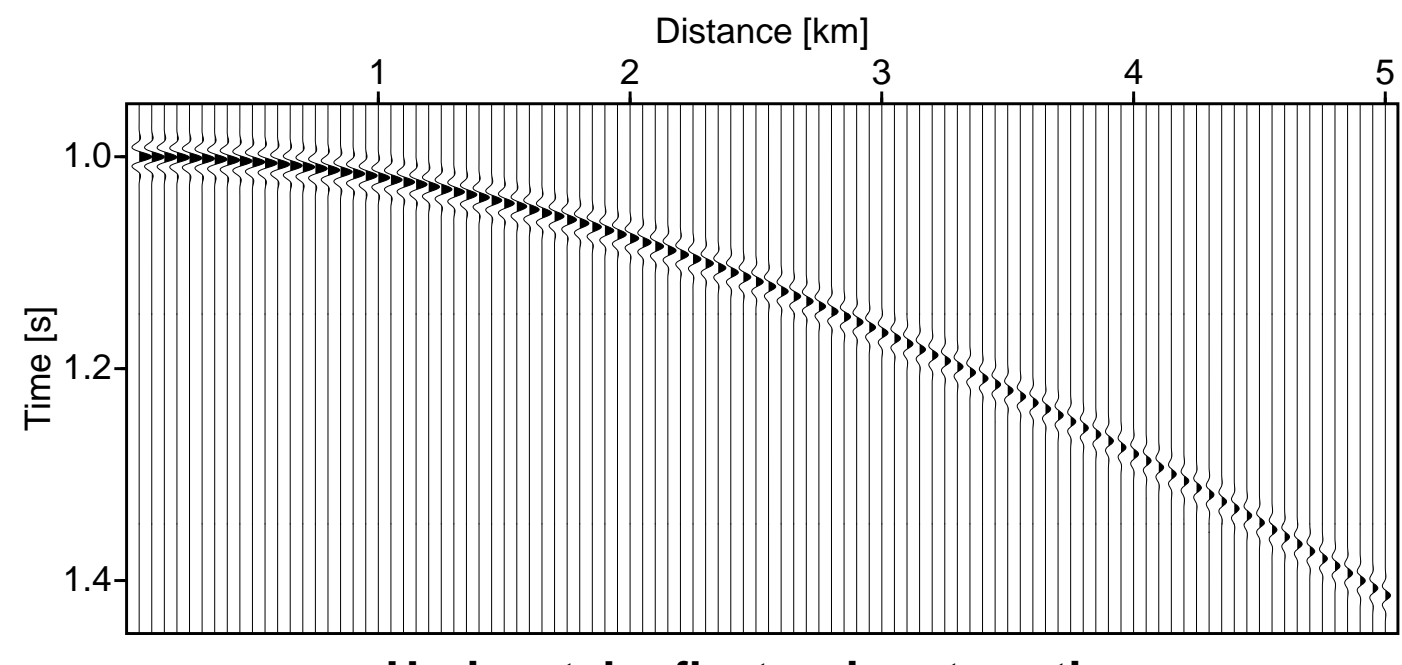

Horizontal reflector: input section

Figure 6.3: Synthetic common shot section for the first example, a two-layer model with a horizontal reflector.

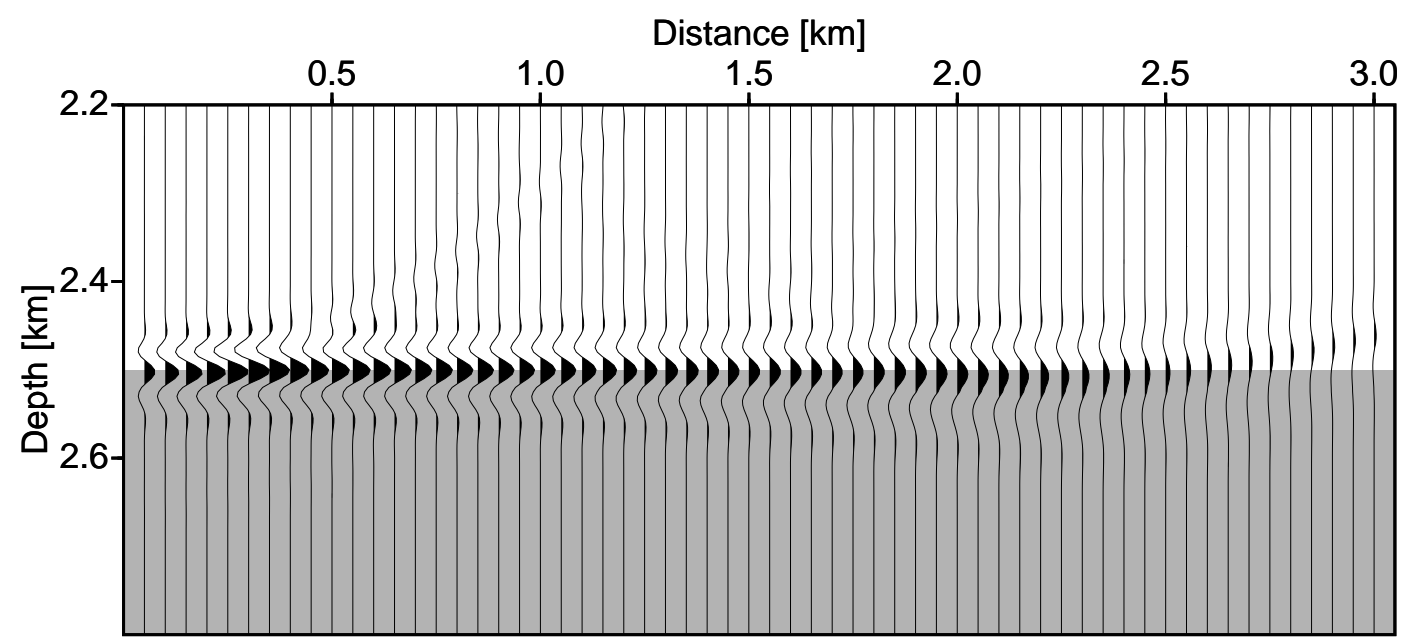

Horizontal reflector: migrated depth section

Figure 6.4: Migrated depth section of the common shot section shown in Figure 6.3. The gray area indicates the lower layer. The reflector was migrated to the correct position and the source pulse, a Gabor wavelet, was reconstructed. 
losses caused by the overburden, the amplitudes of the migrated section coincide with the reflection coefficients. Figure 6.5 (top) shows the accordance between amplitudes picked from the migrated section in Figure 6.4 with theoretical values. Apart from the peaks at $300 \mathrm{~m}$ and $2000 \mathrm{~m}$ distance the two curves coincide, i.e. the correct AVO behaviour was reconstructed. The peaks are aperture effects caused by the limited extent of the receiver line and can be suppressed with a taper. Figure 6.5 (middle) displays relative errors of the reflection coefficients. As a comparison, errors of reflection coefficients that were computed with our migration routine, but using analytic traveltimes as input data instead of FD traveltimes, are also given in Figure 6.5 (middle). Whereas the average error in the reflection coefficients from FD traveltimes is $2 \%$, the error from analytic traveltimes is a magnitude smaller $(0.22 \%$; for both cases only values that are not affected by aperture effects were considered). The higher error for FD traveltimes as input data is due to the systematical errors that are inherent to the Vidale algorithm. Figure 6.5 (bottom) illustrates that the maximum errors in the reflection coefficients coincide with the region of highest errors in the traveltimes.

The second model has the same velocities and densities as the first model, but here a dipping plane reflector with an inclination angle of $14^{\circ}$ separates the two velocity layers. The reflector depth below the source is $2500 \mathrm{~m}$. Ray synthetic seismograms were computed using the SEIS88 package (Červený and Pšenčík, 1984) for 80 receivers with $50 \mathrm{~m}$ distance starting at $50 \mathrm{~m}$ from the point source. In order to ensure causality in the Vidale algorithm, this velocity model had to be 10-fold smoothed. Figure 6.6 shows the common shot section, and Figure 6.7 shows the migrated depth section together with the original reflector position. Again the reflector was migrated to the correct depth and inclination.

As for the first example the reflection coefficients were picked from the migrated section and compared to analytic values. Figure 6.8 (top) shows the result. The peaks at $900 \mathrm{~m}$ and $2500 \mathrm{~m}$ are again due to boundary effects. Figure 6.8 (bottom) displays the relative errors of the picked reflection coefficients. The average errors for reflection coefficients from FD traveltimes and from analytic traveltimes as input are $2.9 \%$ and $0.27 \%$, respectively. In addition to the already discussed errors caused by the FD routine, the higher error compared to the first model can be attributed to the smoothing of the velocity model. Smoothing of $V^{-1}$, as was applied, preserves only the vertical traveltime. Changes in the traveltimes lead to changes in curvature and, thus, to changes in the weight functions. 


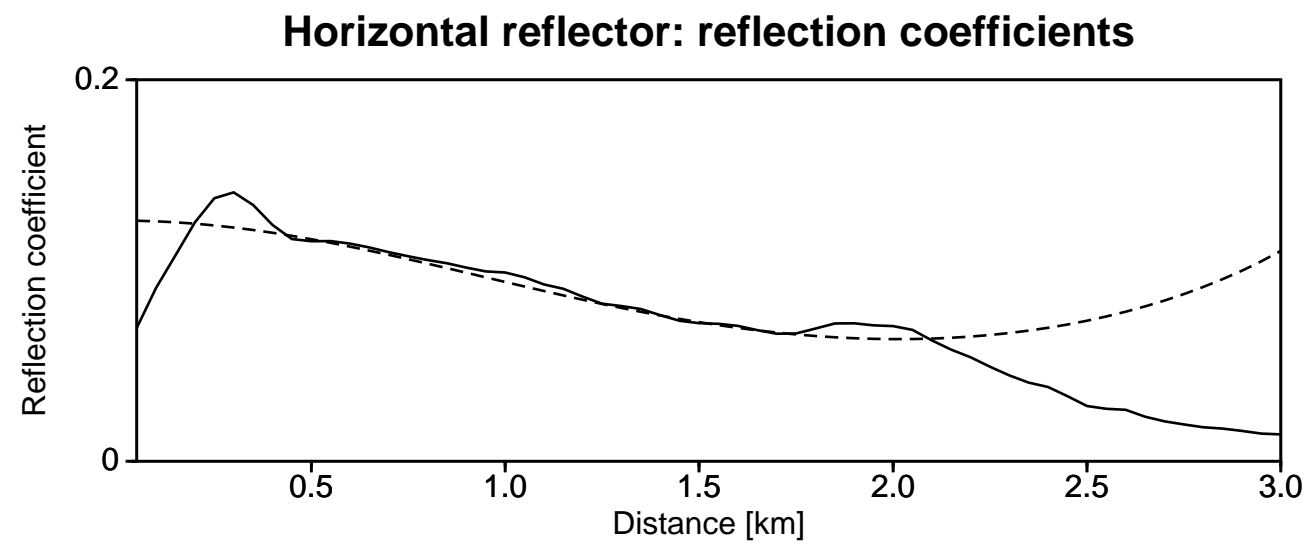

Horizontal reflector: errors in reflection coefficients

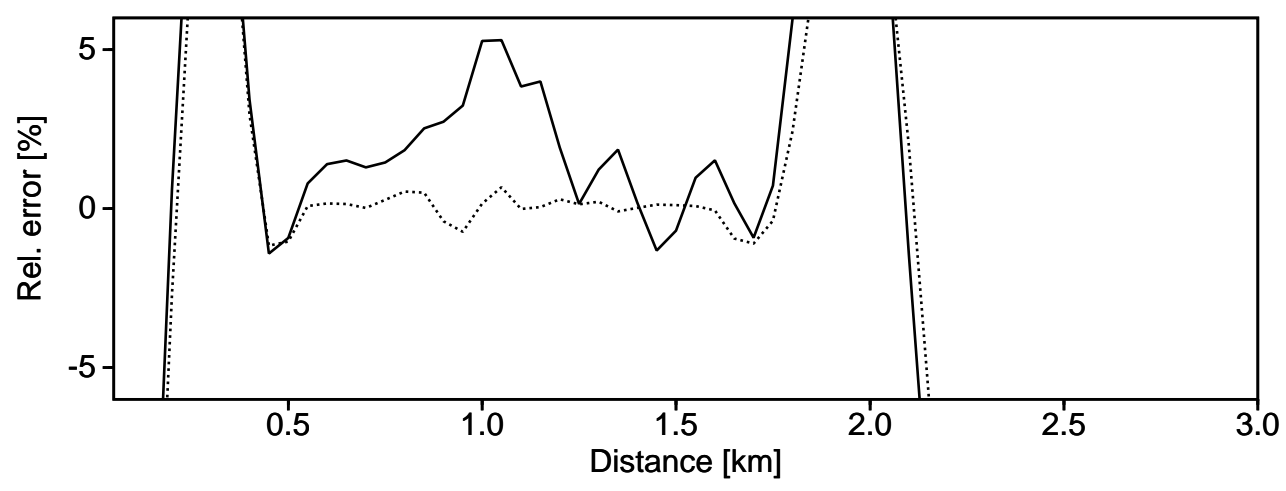

Traveltime errors at depth $2500 \mathrm{~m}$

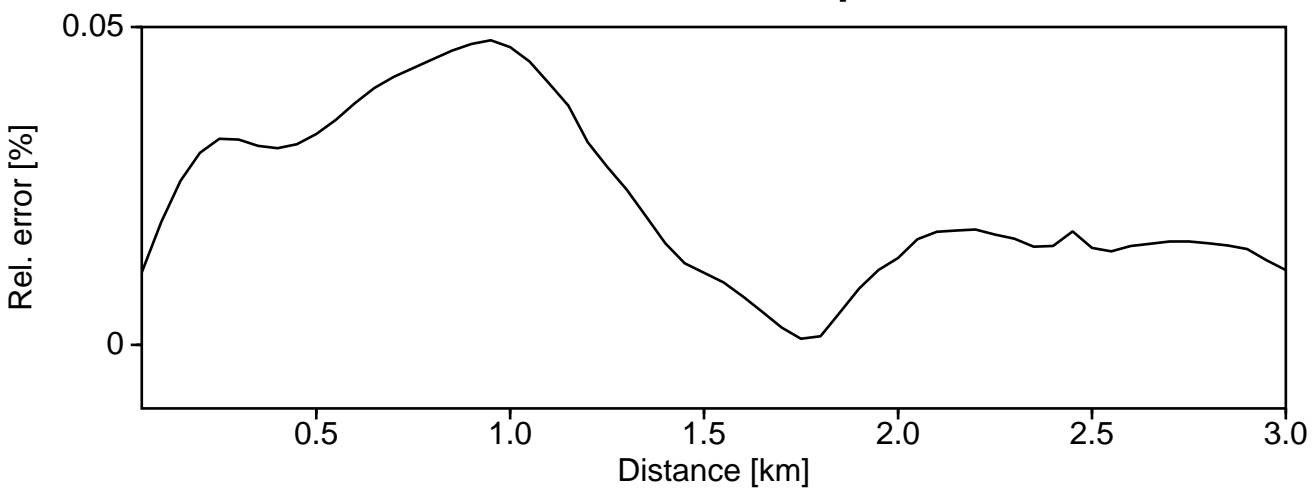

Figure 6.5: Top: solid line: picked reflection coefficients from the migrated section in Figure 6.4; dashed line: analytic values for the reflection coefficients. Middle: solid line: relative errors of the picked reflection coefficients; dotted line: relative errors of reflection coefficients if analytic traveltimes are used as input. Bottom: relative errors of the input traveltimes computed with an FD eikonal solver taken at the reflector at a depth of $2500 \mathrm{~m}$. The maximum traveltime error coincides with the maximum error in the reflection coefficients. 


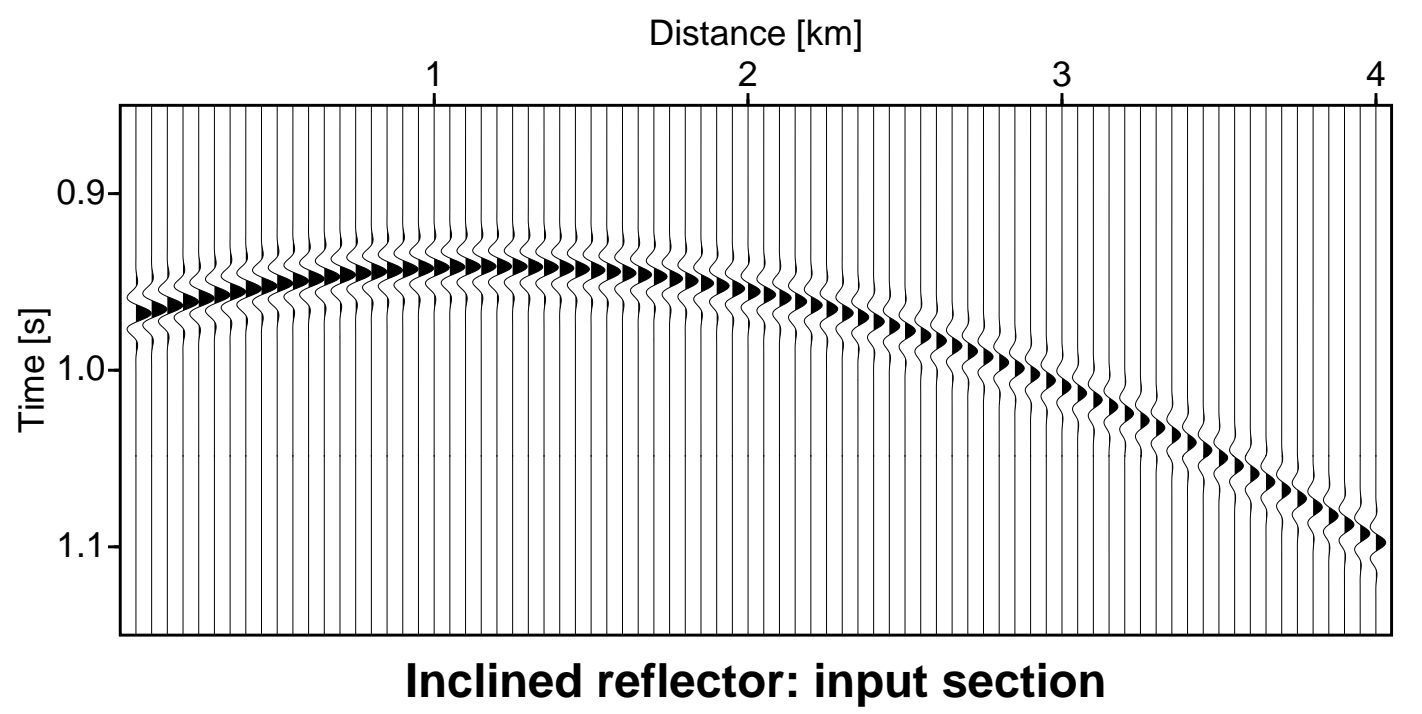

Figure 6.6: Synthetic common shot section for the second example, a two-layers model with an inclined reflector.

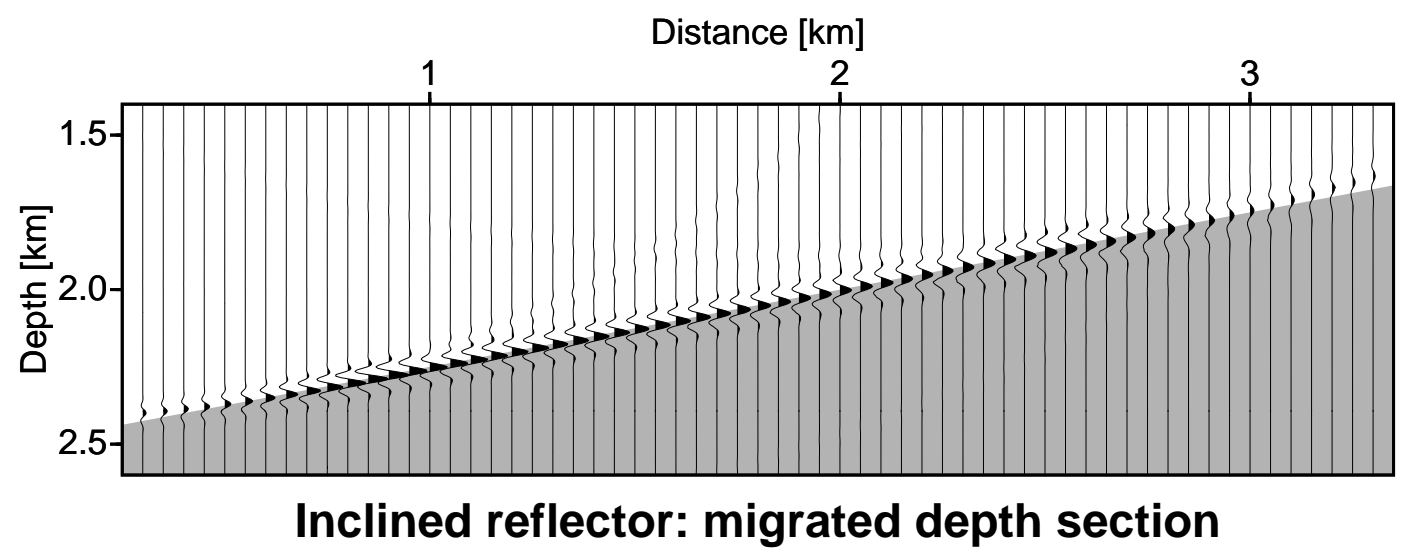

Figure 6.7: Migrated depth section for the second example. The gray area indicates the lower layer. The reflector was migrated to the correct depth and inclination. The source pulse was correctly reconstructed. 


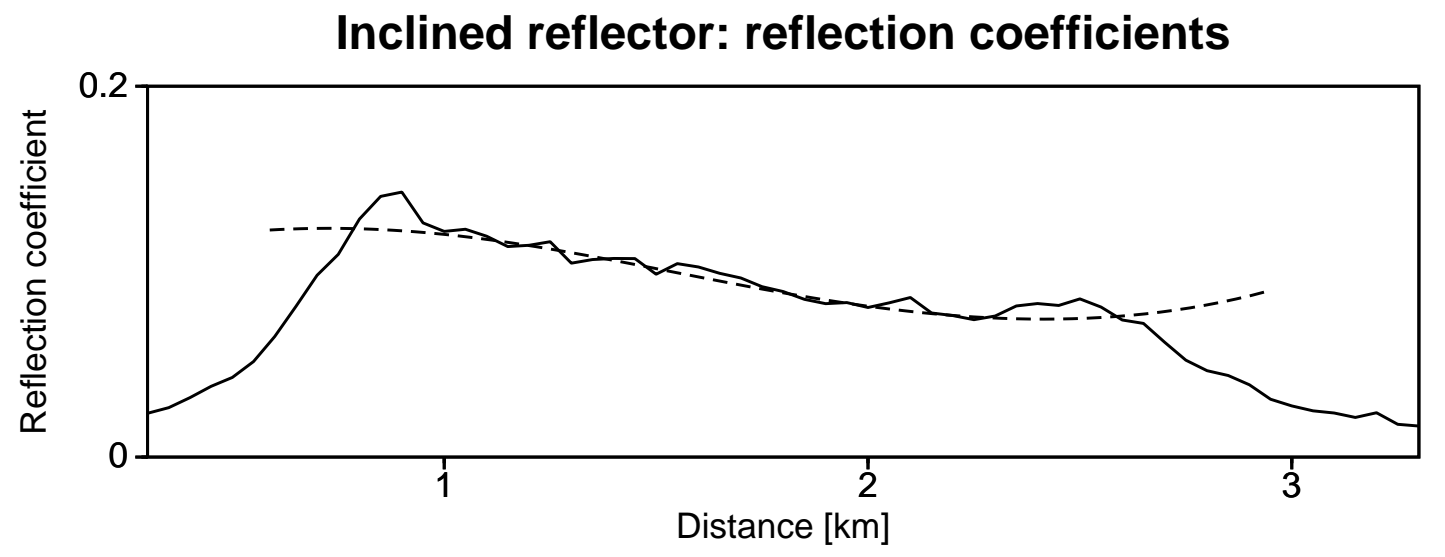

Inclined reflector: errors in reflection coefficients

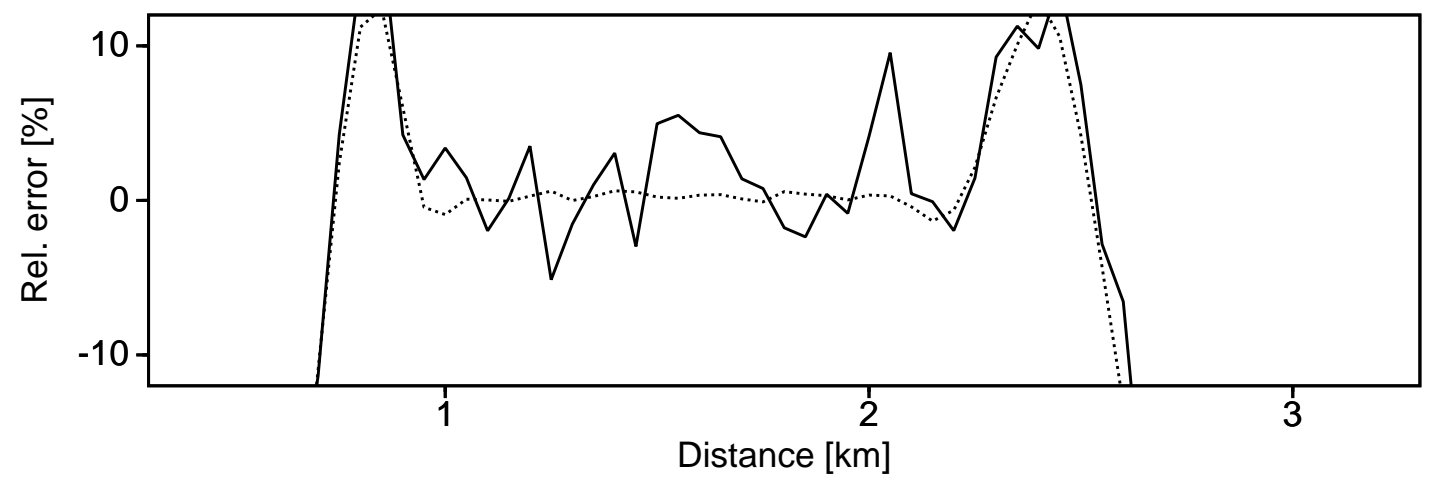

Figure 6.8: Top: solid line: picked reflection coefficients from the migrated section in Figure 6.7; dashed line: analytic values for the reflection coefficients. Bottom: solid line: relative errors of the picked reflection coefficients; dotted line: relative errors of reflection coefficients if analytic traveltimes are used. 


\subsection{Application to PS Converted Waves}

The algorithm can also be applied to PS converted waves. However, an additional factor must be considered in the weight function to allow for the discontinuity of the geometrical spreading at an interface (see Section 2.8, or Červený et al. 1977). For a PS converted wave, the geometrical spreading (6.4.23) becomes

$$
\mathcal{L}=\frac{1}{V_{s}} \sqrt{\frac{\cos \theta_{1}}{\cos \theta_{2}}} \sqrt{\frac{\cos \vartheta_{s} \cos \vartheta_{g}}{|\operatorname{det} \underline{\hat{N}}|}} \mathrm{e}^{-i \frac{\pi}{2} \kappa},
$$

where $\theta_{1}$ is the incidence angle at the reflector and $\theta_{2}$ is the reflection angle. For PP reflections, Snell's law requires that $\theta_{1}=\theta_{2}$, yielding again Equation (6.4.23).

The resulting weight function for PS converted waves reads

$$
W\left(\boldsymbol{\xi}^{*}, M\right)=\frac{1}{V_{s}} \sqrt{\cos \vartheta_{s} \cos \vartheta_{g}} \sqrt{\frac{\cos \theta_{1}}{\cos \theta_{2}}} \frac{\left|\operatorname{det}\left(\underline{\bar{N}}_{1}^{\top} \underline{\Sigma}+\underline{\mathbf{N}}_{2}^{\top} \underline{\Gamma}\right)\right|}{\sqrt{\left|\operatorname{det} \underline{\bar{N}}_{1} \operatorname{det} \underline{\mathbf{N}}_{2}^{\top}\right|}} e^{-i \frac{\pi}{2}\left(\kappa_{1}+\kappa_{2}\right)}
$$

Apart from the additional factor that acknowledges the discontinuity of spreading Equation (6.7.41) looks formally equal to the PP weight function, Equation (6.4.27). Note, however, that for PS converted waves, the matrices must be determined from the appropriate traveltime tables. The diffraction traveltime curve consists of the down-going $\mathrm{P}$ wave and the up-going $\mathrm{S}$ wave. Therefore, two sets of input traveltime tables are required for the construction of the diffraction time surface and the weight functions. Matrix elements carrying the index 1 are computed from the $\mathrm{P}$ wave traveltimes and those with index 2 from $\mathrm{S}$ wave traveltime tables.

As stated before, I have developed an alternative formulation of the weight functions that does not require a priori information on the reflector orientation. This new weight function can also be applied to PS converted waves. Its derivation is given in Appendix E.

I present a 2-D example: ray synthetic seismograms (Figure 6.9) were obtained using the SEIS88 package (Červený and Pšenčík, 1984) for a two-layers model with a horizontal interface in a common shot configuration. The P-velocity is $V_{P_{1}}=5 \mathrm{~km} / \mathrm{s}$ in the upper layer and $V_{P_{2}}=6 \mathrm{~km} / \mathrm{s}$ in the lower layer that lies at a depth of $2 \mathrm{~km}$ below the source. The S-velocities are $V_{S_{i}}=V_{P_{i}} / \sqrt{3}$ and the density is given by $\rho=1.7+0.2 V_{P}$ ( $\rho$ in $\mathrm{g} / \mathrm{cm}^{3}$ and $V_{P}$ in $\mathrm{km} / \mathrm{s}$ ). 300 receivers with a spacing of $10 \mathrm{~m}$ were distributed starting $10 \mathrm{~m}$ away from a line source. Only PS reflections were considered in this example.

The migrated depth section is shown in Figure 6.10. Reflection coefficients were picked from the section and are compared to analytic results. Figure 6.11 shows good accordance between the two curves. The (negative) peak at a distance of $1.8 \mathrm{~km}$ is a boundary effect caused by the limited extent of the receiver line, which provides sufficient illumination of the reflector only for distances smaller than $1.8 \mathrm{~km}$. This also causes the diffraction that shows in the migrated section (cf. Figure 6.10). 


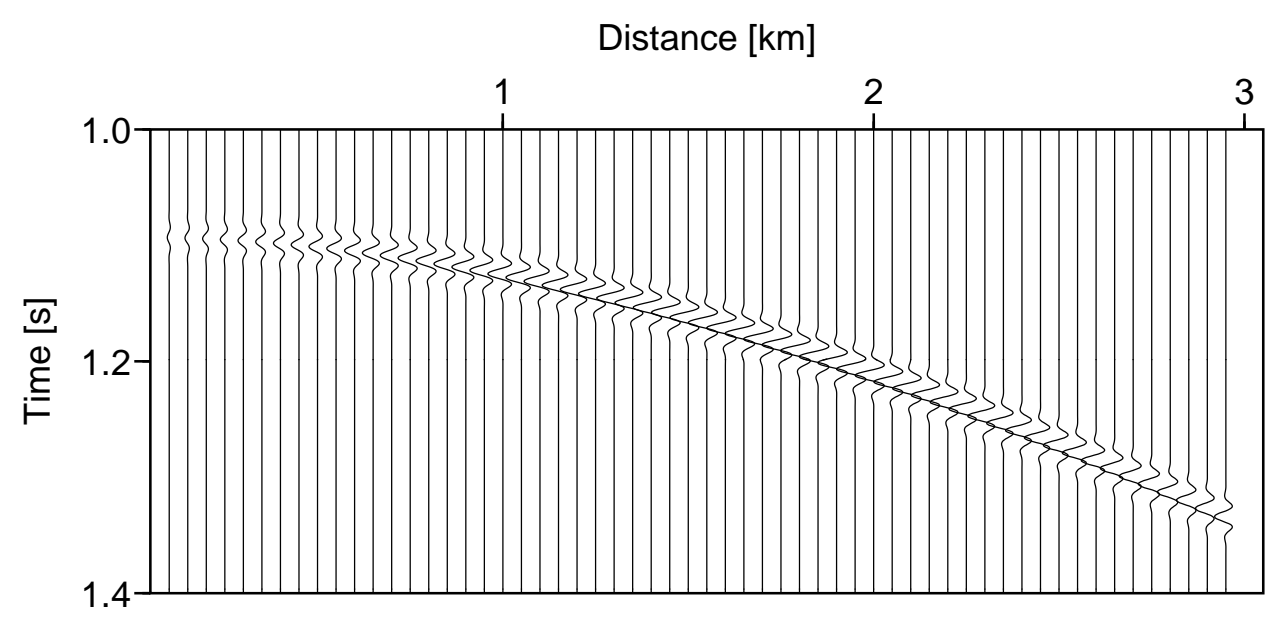

Figure 6.9: Synthetic common shot section: the receiver spacing is $10 \mathrm{~m}$ but only every fifth trace is shown here.

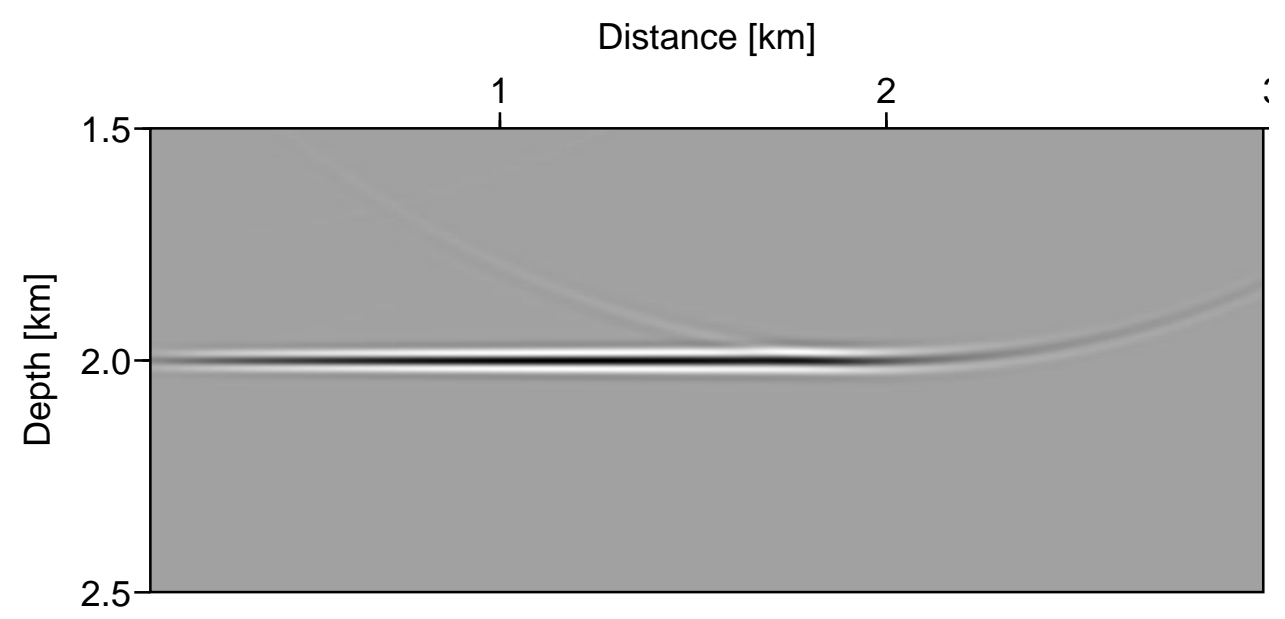

Figure 6.10: Migrated depth section: the reflector was migrated to the correct position. The amplitude matches the reflection coefficient. The diffraction is a boundary effect (see text). 


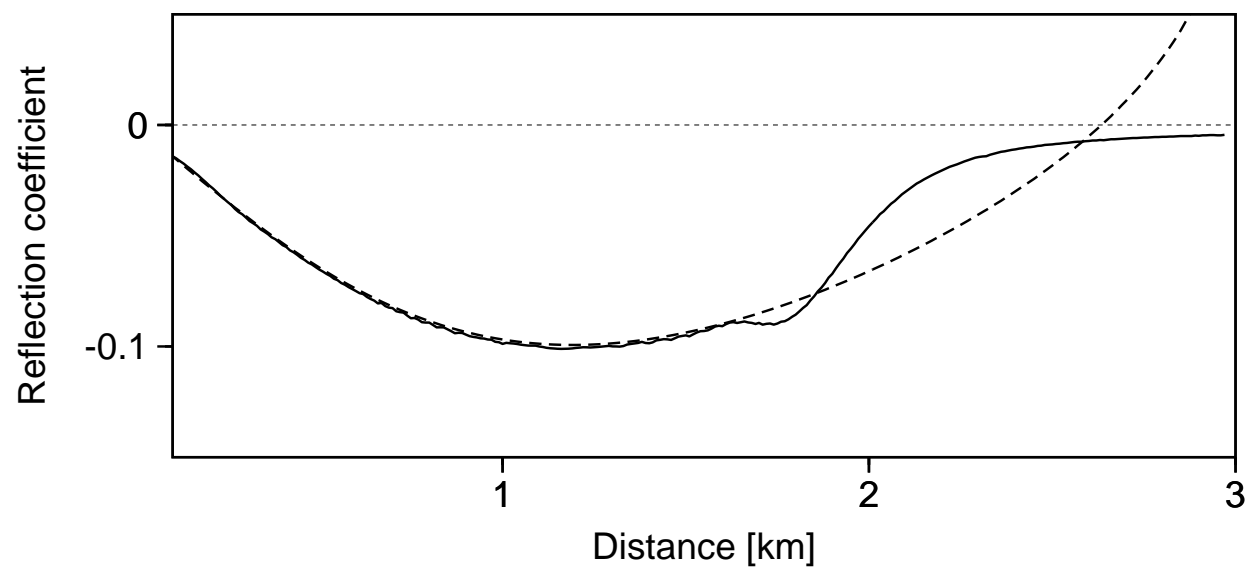

Figure 6.11: Solid line: picked reflection coefficients from the migrated section in Figure 6.10. The peak at $1.8 \mathrm{~km}$ is a boundary effect (see text). Dashed line: analytic values for the reflection coefficients. 


\subsection{Complex Models?}

Of course I would have liked to show results for a complex model as an additional example. Instead, I give the reasons why I did not apply my method to such a model so far. This section also explains why I am confident that the method will nevertheless perform well in complex models.

The reason, why no complex model was used only looks simple on the first view: at the time of finishing this thesis there was no suitable synthetic dataset available for the validation of the method. Going into details reveals some problems, which are, however, not caused by the technique itself. The necessary input information for my migration scheme are

- the velocity model,

- the seismograms,

- the traveltime tables.

In the case of synthetic data, the velocity model is needed for the generation of the seismograms, but it is in any event needed for the computation of the traveltime tables and the traveltime coefficients, i.e. slownesses and second-order derivative matrices. The velocity model also contains information on the inclination and curvature of reflectors. A typical candidate for a complex velocity model would contain several velocity layers as well as a synclinal or anticlinal structure, e.g., a salt dome, and faults since these are the situations that are of most interest for exploration.

Complex structures in the velocity model express themselves in complex seismic sections, that contain later arrivals, and especially triplications. These are of considerable interest, because a lot of energy is contained in their reverse branches. Therefore the reconstruction of amplitudes with a true-amplitude migration makes sense, only if later arrivals are accounted for. This has consequences for the choice of the algorithm which is used for the generation of the traveltime tables. Finite-difference eikonal solvers (FDES) are not suited because they compute only first arrivals. The alternative to FDES are techniques which are based on the ray method. Currently, two such programs are available to the author. The first is based on the wavefront construction method (WFC, Vinje et al. 1993) and part of the NORSAR program package. The second program employs the wavefront oriented ray tracing technique (WRT) developed by Coman and Gajewski (2001).

Figure 6.12 shows (first arrival) traveltimes which were computed for the PICROCOL model (Brokešová et al., 1994) with the NORSAR program package. The relevant part of the model is also given by Figure 6.12. One can clearly see that there are regions where no traveltimes have been computed. These are shadow zones, which were not covered with rays, therefore no traveltimes are available, and the values are set to zero. It is impossible to determine the traveltime coefficients which are required for the weight functions and the diffraction time surface in these regions. This problem with incomplete 


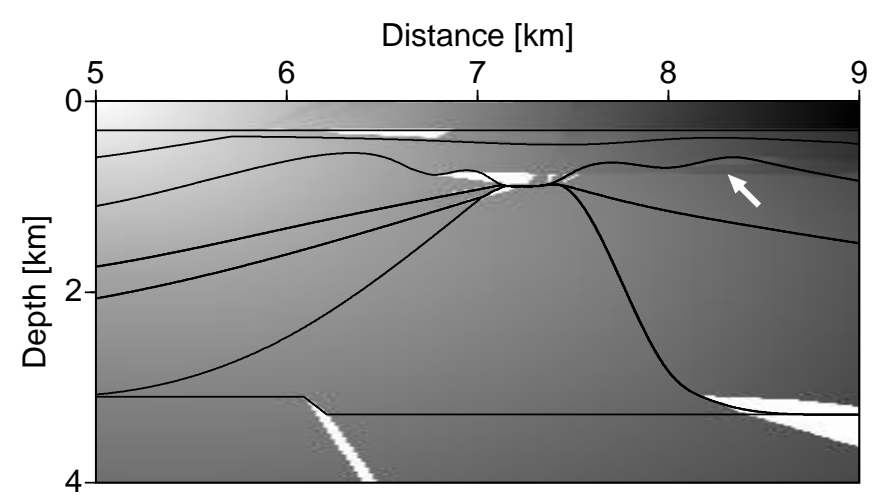

PICROCOL: traveltimes

Figure 6.12: Traveltimes for the PICROCOL model, computed with the NORSAR package. The values of the traveltimes are displayed by the grayscale with white corresponding to zero traveltime. The source is located in the upper left corner of the model. The black lines correspond to the interfaces of the model. White areas are regions, where the traveltime value is zero. These are shadow zones, that could not be covered with rays. In other regions (see the arrow) the traveltimes appear to be wrong, since there seems to be a discontinuity in them. It is possible that this traveltime belongs to a later arrival.

traveltime tables is not inherent to the wavefront construction method, but applies to all ray tracing techniques. The reason is that for models with discontinuities of the velocity - as for the "hard" reflectors in the PICROCOL model - the scale length is no longer large compared to the wavelength (cf. Equation (2.1.7)): the velocity variations take place on a much smaller scale at the interfaces, especially for thin layers. If this is the case, ray tracing techniques can become instable.

To adjust the scale length, smoothing of the velocity model is advised. The technique of Coman and Gajewski (2001) yields complete traveltime tables including later arrivals, if wavenumbers below $200 \mathrm{~m}$ are removed from the velocity model (Gajewski, personal communication). Smoothing of the velocity model, however, leads to deviations of the traveltimes and wavefront curvatures compared to those for the unsmoothed model. Consequently, errors in the weight functions, and therefore in the reconstructed amplitudes occur. I will illustrate this with a simple example.

Consider a reflector with an overburden that consists of two parallel constant velocity layers of equal thickness $d_{1}=d_{2}$ (Figure 6.13). This model has a velocity discontinuity where the velocities $v_{1}$ and $v_{2}$ meet. The extreme case of a smoothed version of this model would be one layer with a constant velocity $v$, where

$$
v=\left(d_{1}+d_{2}\right)\left(\frac{d_{1}}{v_{1}}+\frac{d_{2}}{v_{2}}\right)^{-1}=\frac{1}{2} \frac{v_{1} v_{2}}{v_{1}+v_{2}} .
$$

This form of $v$ arises if slownesses are smoothed instead of velocities. It ensures that the vertical traveltime to a point on the reflector is conserved ${ }^{1}$. Figure 6.14 shows the weight

\footnotetext{
${ }^{1}$ This is the reason, why the slownesses are smoothed instead of velocities. Note, however, that the
} 


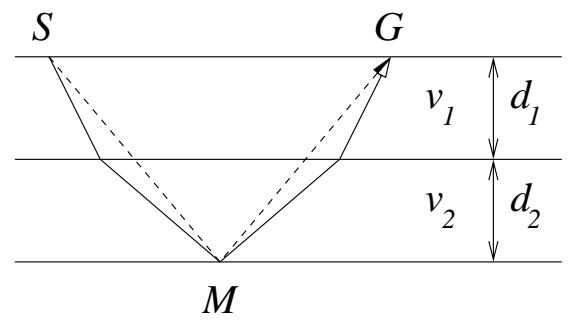

Figure 6.13: Ray paths from a source at $S$ to a receiver at $G$ via a subsurface point $M$ assumed to lie on a reflector. Rays are shown for a two-layer medium (solid line) and a substitute medium (dashed line) with constant velocity. The constant velocity is chosen in such a way that the vertical traveltime is conserved. 
functions for the original model and for the smoothed version. The error of the weights for the smoothed model increases with growing offset. This leads to an underestimation of the recovered reflection amplitude for higher offsets. Although in this example the smoothing was taken to its extreme, the effect has also been shown by Peles et al. (2001) for a model which was only reasonably smoothed.
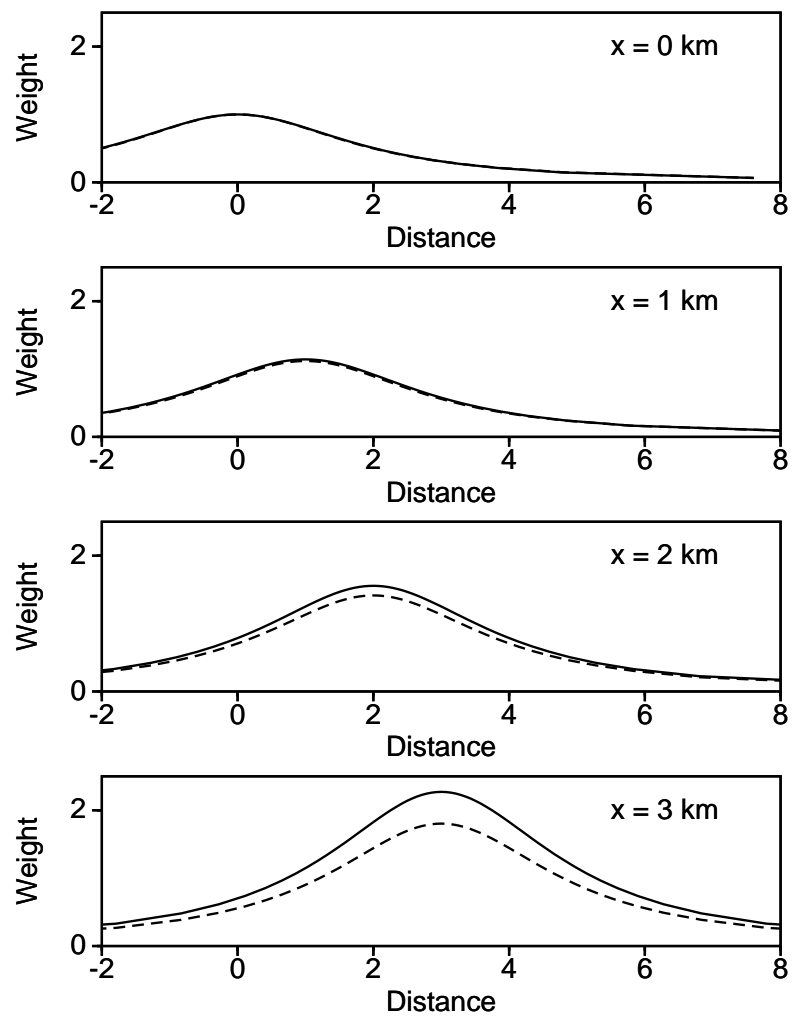

Figure 6.14: Weight functions for a two-layer overburden model as in Figure 6.13 (solid line) and the constant velocity substitute (dashed line) for a point at the distance $x$ on the reflector. The source is located at zero. The error of the weight function which uses the substitute medium increases for growing $x$.

The astute reader may now remark, that although I have reservations against the use of smoothed velocity models, I employ a different kind of smoothing myself for the weight functions: every interpolation corresponds to smoothing. Since the weight functions are determined at the coarse grid points only, trilinear interpolation is carried out onto all fine grid subsurface points, thereby smoothing the weight functions. The observation that this leads to errors in the weight functions is correct. However, the effect is small, as I will demonstrate with a simple example.

Figure 6.15 shows a two-layer medium with a horizontal interface at the depth $z$. It

conservation of the vertical traveltime applies only to one depth point under the source at the depth $z_{0}$, if the model was smoothed between $z=0$ and $z_{0}$. E.g., for a point in the $v_{1}$-layer the vertical traveltime is $z / v_{1} \neq z / v$, if $v$ was determined from the $v_{i}$ until reflector depth! 


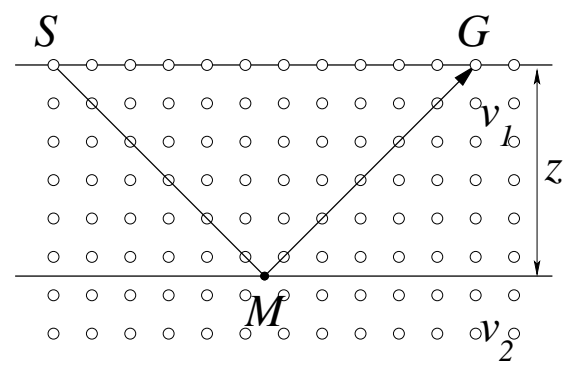

Figure 6.15: Representation of a two-layer medium. The circles denote coarse grid points where the weight functions are determined. At the depth $z$ the weights need to be interpolated onto the reflector. This leads to errors.

is unlikely that the reflector will coincide with the coarse grid points at which the weight functions are determined. This situation is given in Figure 6.15. The correct, un-interpolated weight functions would be determined in the reflector surface. This means that only traveltimes to points on the reflector would enter the computation, which are in this case those for a constant velocity model with velocity $v_{1}$. Since traveltimes are not available in the reflector surface but only in planes below and above it, the weights for the points on the reflector are interpolated from the points below and above. In the model case the weights above the reflector are the correct ones since the velocity is constant $v_{1}$. Therefore the traveltimes from which the weights are computed are the correct ones. Below the reflector, the traveltimes behave different due to the discontinuous change in slowness at the interface, and the change in wavefront curvature (see, e.g., Leidenfrost et al. 1998). The weights at this depth are derived from traveltimes which were obtained by using velocity $v_{2}$ for the grid points below the reflector. They are, therefore, wrong with respect to the reflector. As a consequence, the weights on the reflector which result from interpolation of the values below and above the reflector are also wrong.

The errors in the weight functions which result from the interpolation of weight functions from below and above the reflector are very small. Figure 6.16 shows the weight functions and the relative errors for a model like the one in Figure 6.15 with $v_{1}=4.5 \mathrm{~km} / \mathrm{s}$, $v_{2}=5 \mathrm{~km} / \mathrm{s}$, and $z=2 \mathrm{~km}$. The coarse grid spacing in this example is $100 \mathrm{~m}$, with the reflector in the middle between two coarse grid points. Only for higher offsets the errors increase. The move-out is smaller there, therefore the curvature in terms of the matrix $\underline{\bar{N}}$ is small, as is the weight function. This lets the errors appear higher since they are relative errors.

Although for the given reason smoothed velocity models are not perfectly suited for this technique of true-amplitude migration, I assume that they would nevertheless lead to satisfactory results. They are, however, not appropriate for the validation of the technique with respect to a complex model. I have applied the technique to simple models so far to be able to validate the method itself. The use of input data, which, itself, leads to errors in the determination of the weight functions can not serve the purpose of validation of the technique. Therefore, the technique has not yet been applied to a complex model. 

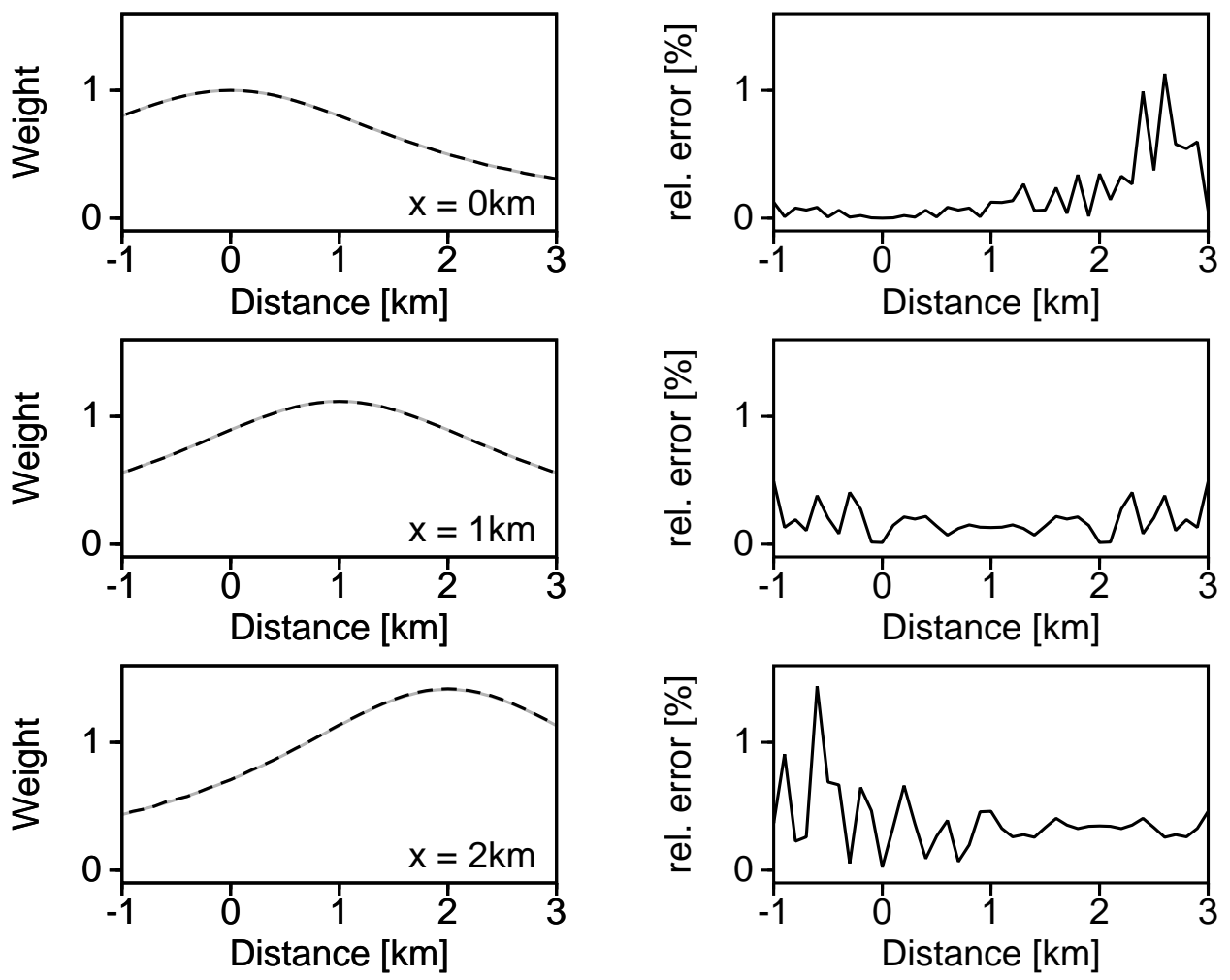

Figure 6.16: Weight functions for three points at the distance $x$ on the reflector for the model given by Figure 6.15. Right: analytic weight function (gray lines) and interpolated weight function (dashed lines). Since both curves nearly coincide, the relative errors of the interpolated weight functions are given on the left. The slightly increasing error towards larger distance from the image point is caused by the smaller values of the weight function.

There are other means, though, to assess the applicability of the method to complex models. The traveltime coefficients which are used for the computation of the weight functions are the same as in the expressions for the geometrical spreading, as well as for the traveltime interpolation. The high accuracy of the traveltime interpolation and the spreading computation has already been discussed in Chapter 3 and Chapter 5, also with respect to a complex model, the Marmousi model. These results are cause for my trust that the migration technique will perform as well for complex models as the computation of spreading and traveltimes. 


\section{Chapter 7}

\section{Limited Aperture Migration}

True amplitude migration as introduced in Chapter 6 is based on a summation stack along diffraction time surfaces. If the summation is carried out over the whole aperture of the experiment, this becomes a very time consuming process. A significant part of this time, however, is spent unnecessarily: traces where the diffraction time and the traveltime of the associated reflected event differ by more than the duration of the source pulse (this criterion defines the minimum migration aperture), do not contribute to the desired migration result, but only lead to an increase in migration noise. Thus, a restriction to the minimum aperture as an optimised migration aperture can significantly enhance the image quality as well as the computational efficiency. Also, once the minimum aperture is determined, boundary effects can be recognised as such.

Although it is a different physical concept, the definition of the optimised (minimum) aperture bears a strong formal relationship to the (first) Fresnel zone, the intersection surface of the Fresnel volume with the reflector surface. In 1992, Cervený and Soares propose an algorithm for Fresnel volume ray tracing. They "believe that Fresnel volume ray tracing will find [...] applications [...] in the inversion of seismic data in the near future". Hubral et al. (1993a) describe the Fresnel zone by paraxial approximation in terms of second order derivative matrices of traveltimes. For the zero-offset situation the projection of this paraxial Fresnel zone onto the earth's surface is given by Hubral et al. (1993b). Schleicher et al. (1997) derive an expression for the projected Fresnel zone for arbitrary measurement configurations. They also introduce an expression for the size of the optimum migration aperture, which is also derived using the paraxial approximation. They further show that in this approximation both projected Fresnel zone and optimum migration aperture coincide.

Schleicher et al. (1997) compute the optimum migration aperture by means of dynamic ray tracing. The quantities that are required are, however, as the weight functions, related to second-order traveltime derivatives. Therefore, the optimum aperture can be determined from traveltimes. In the first section an approximate expression for the optimum migration aperture will be derived. I will show that the quantities which determine the optimum aperture are the same as the matrix elements used in the weight functions and traveltime interpolation. I will give examples on limited aperture migration in the section thereafter. 
The chapter concludes with an estimate of possible savings in computational time.

\subsection{Optimum Migration Aperture}

Figure 7.1 shows an extract of a seismic section for a single reflector and a diffraction traveltime curve for a point on the reflector. One can easily see that only those traces contribute to the stack (6.2.3) where the diffraction traveltime curve $\tau_{D}$ is within the reflection traveltime $\tau_{R}$, and $\tau_{R}$ plus the duration of the signal, $\tau_{L}$. As in Chapter 6 which introduces amplitude-preserving migration it is assumed that the seismic experiment is designed to provide sufficient illumination of the target area. Schleicher et al. (1997) use this criterion to define the minimum aperture, which is also the optimum aperture:

$$
\left|\tau_{F}\right|=\left|\tau_{D}-\tau_{R}\right| \leq \tau_{L}
$$

where $\tau_{F}$ is the difference between diffraction and reflection traveltime.Please note that the terms minimum and optimum aperture as introduced by Schleicher et al. (1997) refer to the reconstruction of the amplitude. Sun (1999) uses the same terms but with respect to elimination of a specific type of migration noise. Therefore the optimum apertures of Schleicher et al. (1997) and Sun (1999) do not coincide. I use the term "optimum" aperture in the sense of Schleicher et al. (1997).

Equation (7.1.1) bears a strong similarity to the definition of the (first) Fresnel zone. The difference between the two concepts is, that the Fresnel zone is defined on the reflector, whereas the minimum aperture criterion is applied in the registration surface. Figure 7.2 demonstrates this difference.

To find an expression for the optimum aperture as it is given by Equation (7.1.1), $\tau_{F}$ is expanded into a second-order Taylor series with respect to the 2-D source-receiver location coordinates $\boldsymbol{\xi}=\left(\xi_{1}, \xi_{2}\right)$. The expansion is centred at the stationary point $\boldsymbol{\xi}^{*}$, where $\tau_{D}=\tau_{R}$ and $\vec{\nabla} \tau_{F}=0$ :

$$
\tau_{F}=\frac{1}{2}\left(\boldsymbol{\xi}-\boldsymbol{\xi}^{*}\right)^{\top} \underline{\boldsymbol{H}}_{\mathrm{F}}\left(\boldsymbol{\xi}-\boldsymbol{\xi}^{*}\right)
$$

in which $\underline{\mathrm{H}}_{\mathrm{F}}$ is the Hessian matrix of the traveltime difference $\tau_{F}$. It has already been introduced in Section 6.3, where it was required for the weight functions. With this equation and Equation (7.1.1), the optimum aperture in paraxial approximation is given by (Schleicher et al., 1997):

$$
\frac{1}{2}\left|\left(\boldsymbol{\xi}-\boldsymbol{\xi}^{*}\right)^{\top} \underline{\mathrm{H}}_{\mathrm{F}}\left(\boldsymbol{\xi}-\boldsymbol{\xi}^{*}\right)\right|=\tau_{L}
$$

For the evaluation of Equation (7.1.3), however, the centre of the aperture that corresponds to the stationary ray must be known. This can be determined from the slowness vectors. As I assume the velocity model to be known, information on the inclination angle of the reflector is available. The incidence angle $\theta_{1}$ on the reflector is then given by

$$
\cos \theta_{1}=\sqrt{1-V_{1}^{2} \overline{\boldsymbol{q}}_{1} \cdot \overline{\boldsymbol{q}}_{1}}
$$




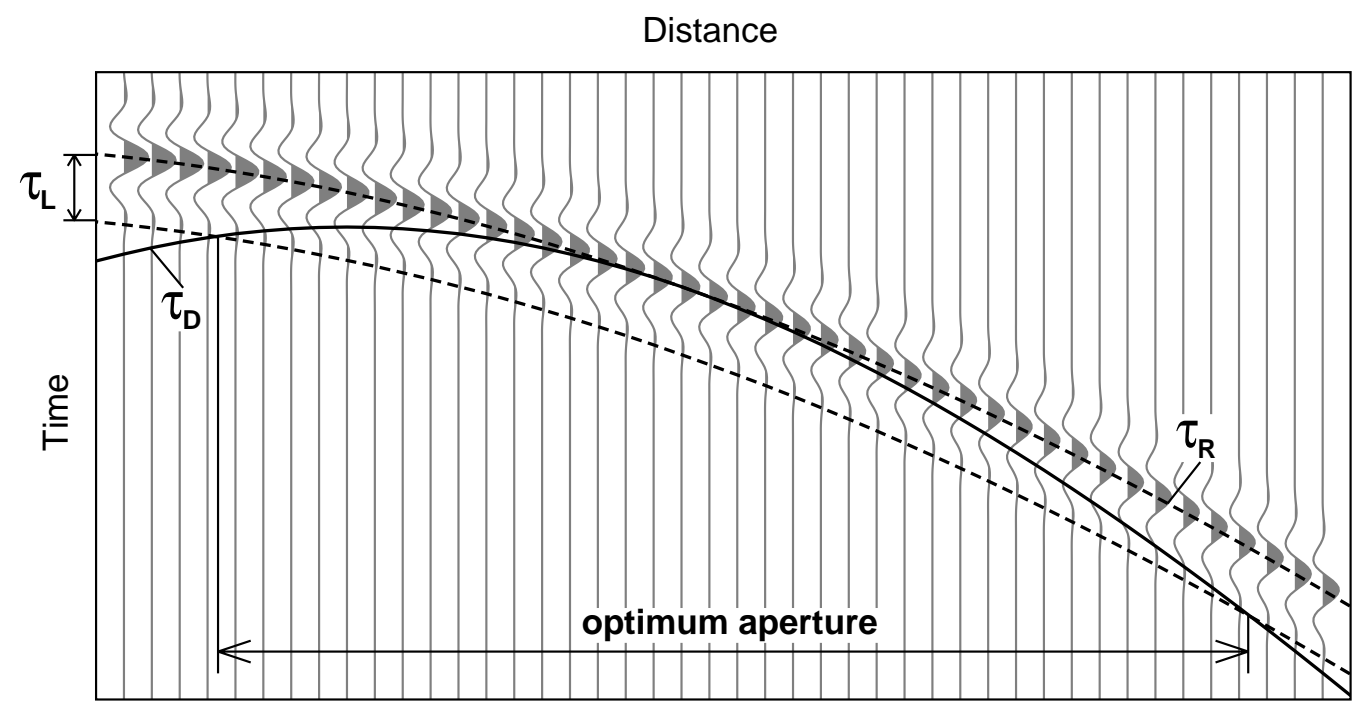

Figure 7.1: Schematic seismic section for a single reflector and traveltime curve for a diffraction point on the reflector. Dashed lines: reflection traveltime $\tau_{R}$ (coinciding with the events) and $\tau_{R}+\tau_{L}$, where $\tau_{L}$ is the duration of the source pulse. Solid line: diffraction traveltime $\tau_{D}$. Traces outside the optimum aperture, which is given by the intersection points between the diffraction curve and the "end" of the signal, do not contribute to the diffraction stack.

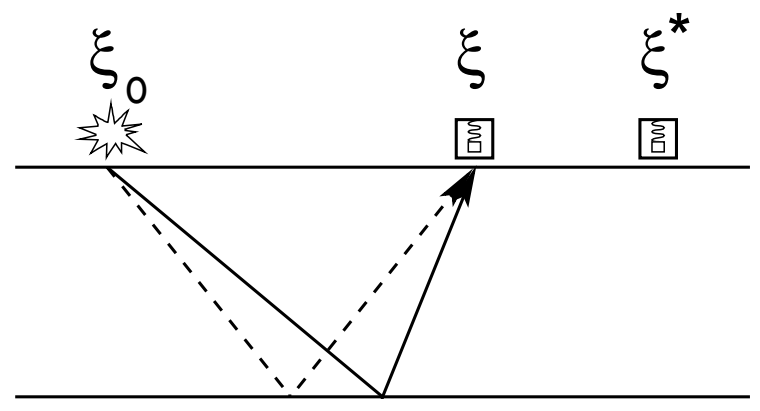

$\mathbf{R} \mathbf{R}^{*}$

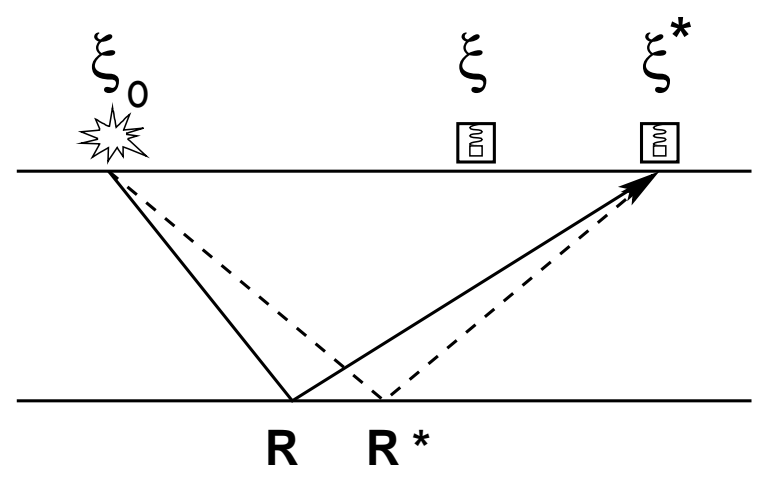

Figure 7.2: Minimum migration aperture (left) and Fresnel zone (right): the criterion for the minimum aperture (in the $\xi$-surface) is that the traveltimes $\tau\left(\overline{\xi_{0} R^{*} \xi}\right)$ and $\tau\left(\overline{\xi_{0} R \xi}\right)$ do not differ by more than the signal length $\tau_{L}$. The Fresnel zone on the reflector is defined such that the difference between the traveltimes $\tau\left(\overline{\xi_{0} R^{*} \xi^{*}}\right)$ and $\tau\left(\overline{\xi_{0} R \xi^{*}}\right)$ is smaller than half a period $T / 2$. The stationary ray is from $\xi_{0}$ to $\xi^{*}$ passing $R^{*}$ in both cases. Dashed lines indicate reflected rays and solid lines stand for diffracted rays. 
where $\overline{\boldsymbol{q}}_{1}$ is the slowness vector of the ray from the source to the reflector. Similarly, $\overline{\boldsymbol{q}}_{2}$ is the slowness vector of a ray from the receiver to the reflector. The reflection angle $\theta_{2}$ is expressed by an equivalent of (7.1.4) but using $\overline{\boldsymbol{q}}_{2}$ instead. Since the stationary ray obeys Snell's law, the centre of the aperture for PP data is the geophone position where the difference between $\theta_{1}$ and $\theta_{2}$ is minimal.

As for the weight functions the matrices $\overline{\mathbf{N}}_{I}$ and $\underline{\underline{G}}_{I}$ which are needed for the computation of $\underline{\mathrm{H}}_{\mathrm{F}}$ can be determined from $\underline{\hat{\mathrm{N}}}_{I}$ and $\underline{\hat{G}}_{I}$ by rotating the latter onto the tangent planes of the recording surface and the reflector candidate. Moreover, the curvature of the recording surface and the reflector has to be considered. This procedure is described in Chapter 4. To do so, I assume that a priori information on the velocity model is available. For simple models the inclination of a reflector can be computed from the gradient of the velocity model. More generally, for example if the velocity model is smooth, it can be extracted from a previous migration. The determination of the reflector curvature follows similar lines. For image points that are not located on a reflector, this information will not be available. For these cases, however, the inclination and curvature are irrelevant, because the migration output will be negligible, regardless of inclination and curvature. For simplicity, I assume both to be zero for those points. If the reflector's inclination but not its curvature can be determined for a point which is located on the reflector, I expect that the approximation of the reflector by a locally plane surface will yield satisfactory results in many cases. This assumption appears to be acceptable since applicability of the ray method requires that the radius of reflector curvature is large compared to the wavelength. Moreover, the recovered reflection coefficient is a plane wave reflection coefficient.

\subsection{Examples}

I have applied the algorithm to simple generic models. These have the advantage of known analytic solutions for the involved quantities. Therefore they are very useful for the validation of the method. The example given here is a two-layer model with an inclined interface. The inclination angle is $63^{\circ}$ and the model has a velocity of $5 \mathrm{~km} / \mathrm{s}$ above and $6 \mathrm{~km} / \mathrm{s}$ below the reflector. A ray-synthetic common-shot section was computed with the SEIS88 package (Červený and Pšenčík, 1984) using a Gabor wavelet with a signal length of $25 \mathrm{~ms}$ and $10 \mathrm{~m}$ distance between the receivers. The migration was carried out first using the original noise-free data, and then for the same dataset with white (random) noise added, having a signal-to-noise ratio of 2 . The noisy input section is shown in Figure 7.3. In both cases the optimum aperture was applied as well as the complete aperture for comparison. In limiting the aperture one must, however, take into account that boxcar filtering produces undesirable effects like ringing or overshooting in the migrated image. Therefore a taper was applied at the endpoints of the aperture. Please note that the taper was not applied in the sense of Sun (1999), but to serve a basic rule of signal processing.

Figure 7.4 shows the recovered reflection coefficients from the noise-free section and 


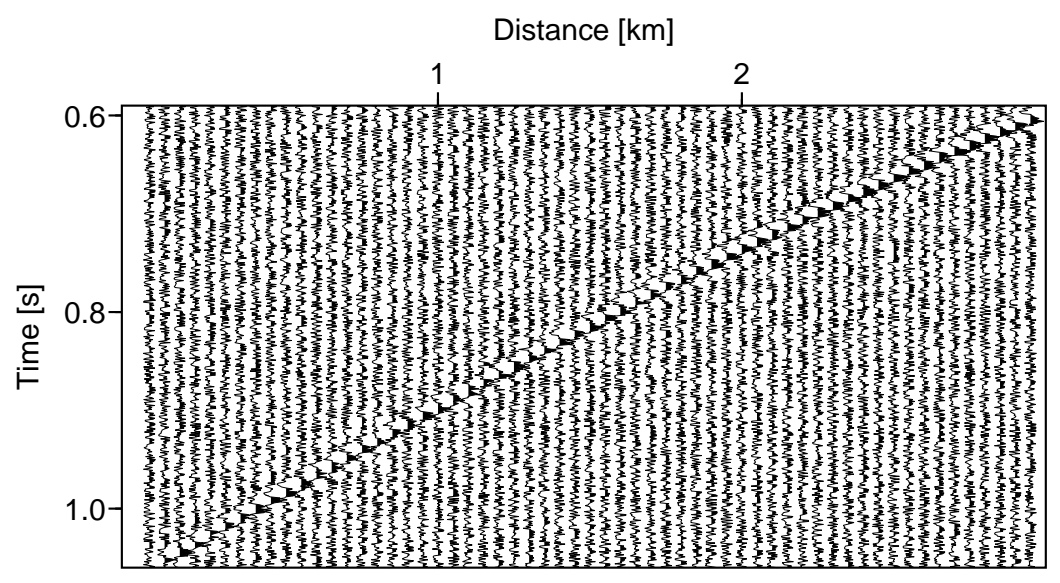

Figure 7.3: Synthetic common-shot section for a $63^{\circ}$ inclined reflector: The receiver spacing is $10 \mathrm{~m}$ but only every fifth trace is shown here. The signal-to-noise ratio is 2 .

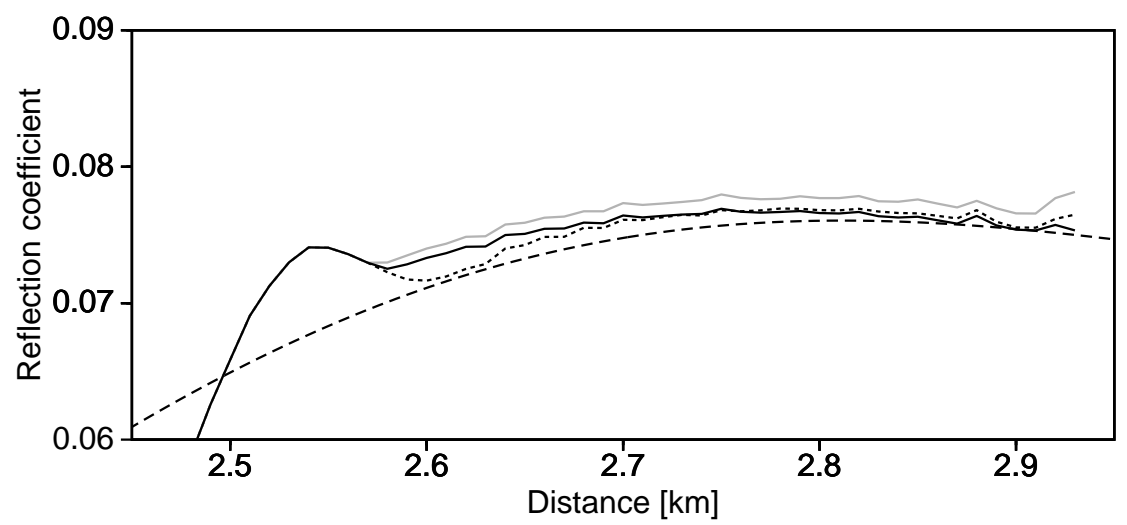

Figure 7.4: Recovered reflection coefficients from noise-free data for a $63^{\circ}$ inclined reflector: Dashed line: analytic reflection coefficients. Dotted line: recovered coefficients if the whole aperture is used. Solid lines: recovered coefficients if the optimum aperture is used. The black line results from applying a taper to the aperture, the gray line follows without tapering. The reflection coefficients from the optimised aperture migration are overestimated (see text). The peak near $2.5 \mathrm{~km}$ is a boundary effect caused by insufficient illumination of the reflector due to the limited extent of the receiver line. 


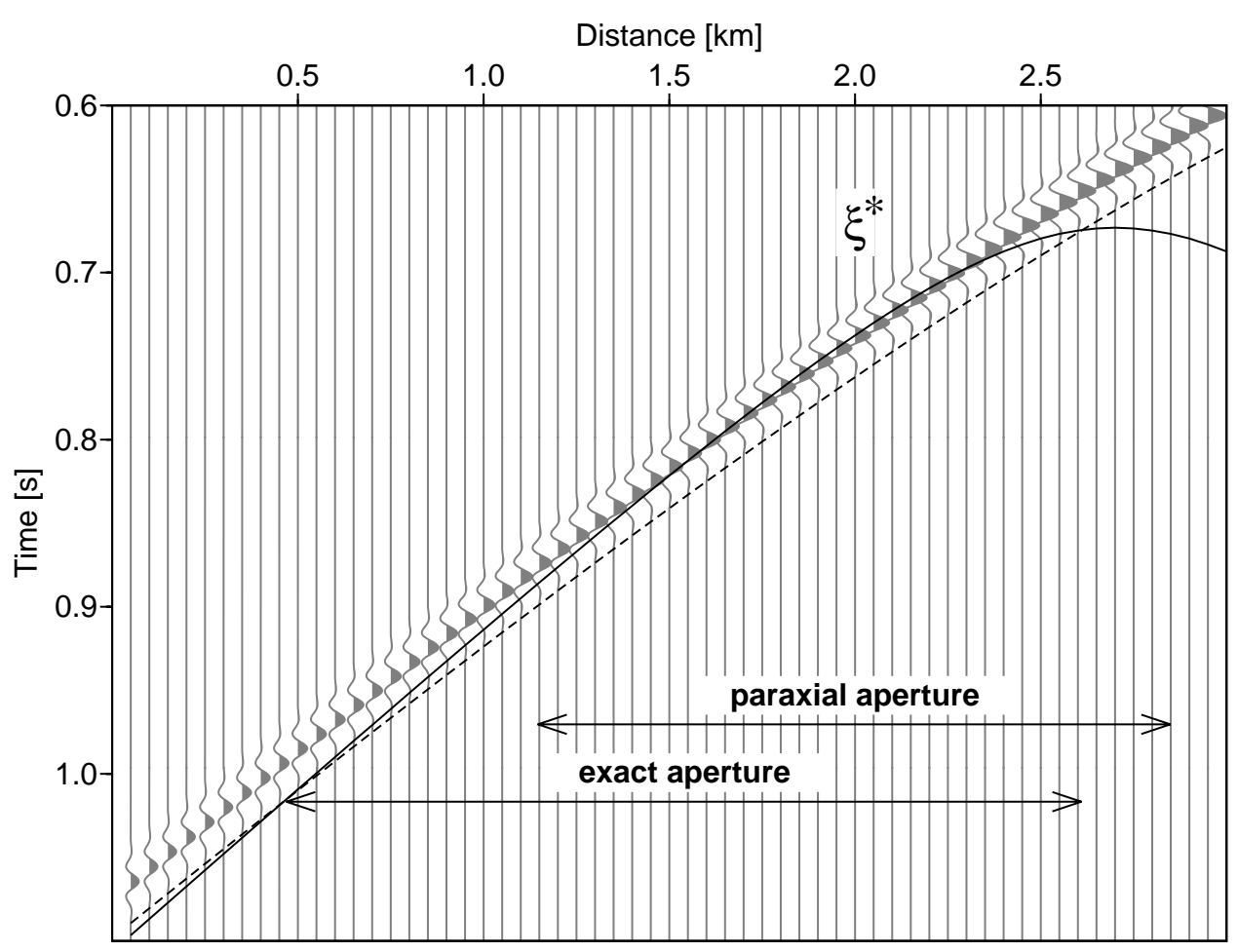

Figure 7.5: Exact and paraxial optimum migration aperture for a point on the $63^{\circ}$ inclined reflector. Dashed line: reflection traveltime plus signal length $\tau_{R}+\tau_{L}$. Solid line: diffraction traveltime curve $\tau_{D}$. The centre of the paraxial aperture that corresponds to the stationary ray is located at $\xi^{*}$.

analytic values. Compared to the whole aperture result, application of the optimum aperture leads to slightly overestimated reflection coefficients. This effect is more pronounced if the taper is omitted. For example, at $2.7 \mathrm{~km}$ distance the error of the recovered reflection coefficient is $1.3 \%$ if the whole aperture is used, it is $1.7 \%$ for the tapered optimum aperture and 3.0\% for the optimum aperture without taper. The reason for the overestimation of the reflection coefficients is that Equation (7.1.3) represents an approximation for the optimum aperture. Figure 7.5 demonstrates the difference between the exact optimum aperture (which can be computed for this type of model, but closed form solutions do not exist for arbitrary models) and the optimum aperture in paraxial approximation resulting from Equation (7.1.3). Please observe in Figure 7.5 that traces within the required aperture, but not within the paraxial aperture would give negative contributions to the stack. This is the reason why the reflection coefficients are overestimated. By applying the taper, additional traces outside of the paraxial aperture are allowed to contribute. This leads to a partial compensation of the overestimation caused by the errors of the paraxial aperture.

The AVO behaviour, however, by which I signify the gradient or the general shape of the AVO is of more interest for interpretation than the absolute value of the reflection coefficients. It is less affected by the effect described above than the absolute value: 


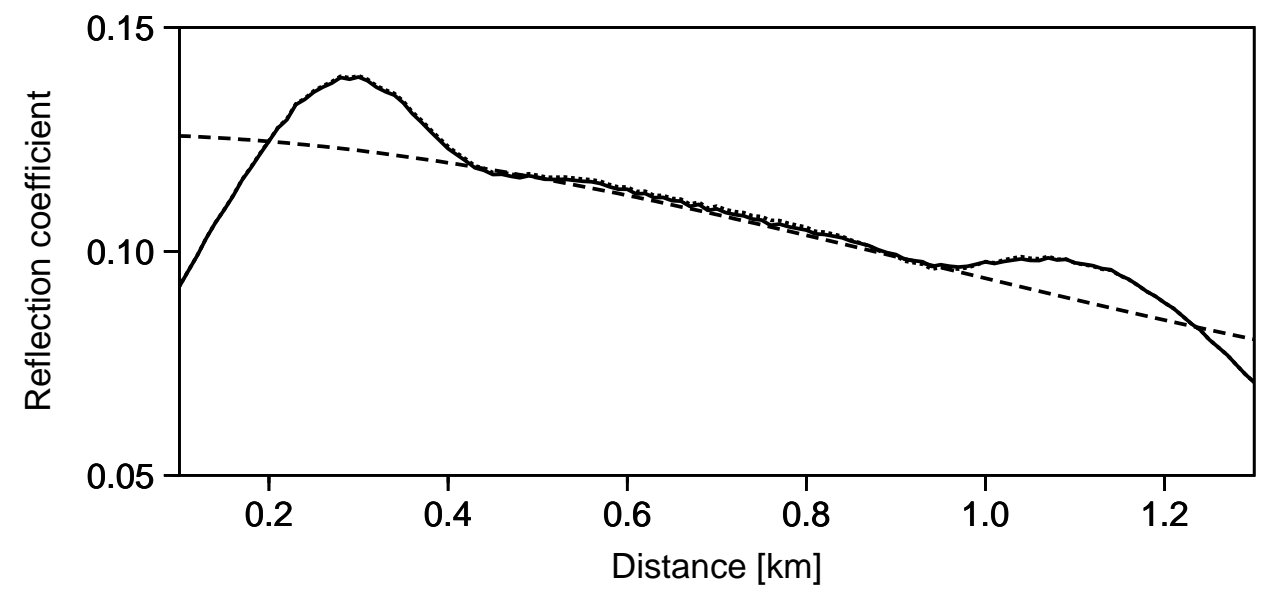

Figure 7.6: Recovered reflection coefficients from noise-free data for a horizontal reflector. The difference between the two results is very small. Dashed line: analytic reflection coefficients. Solid line: recovered coefficients if the optimum aperture is used. Dotted line: recovered coefficients if the whole aperture is used. The two peaks at 0.3 and $1.1 \mathrm{~km}$ are again boundary effects.

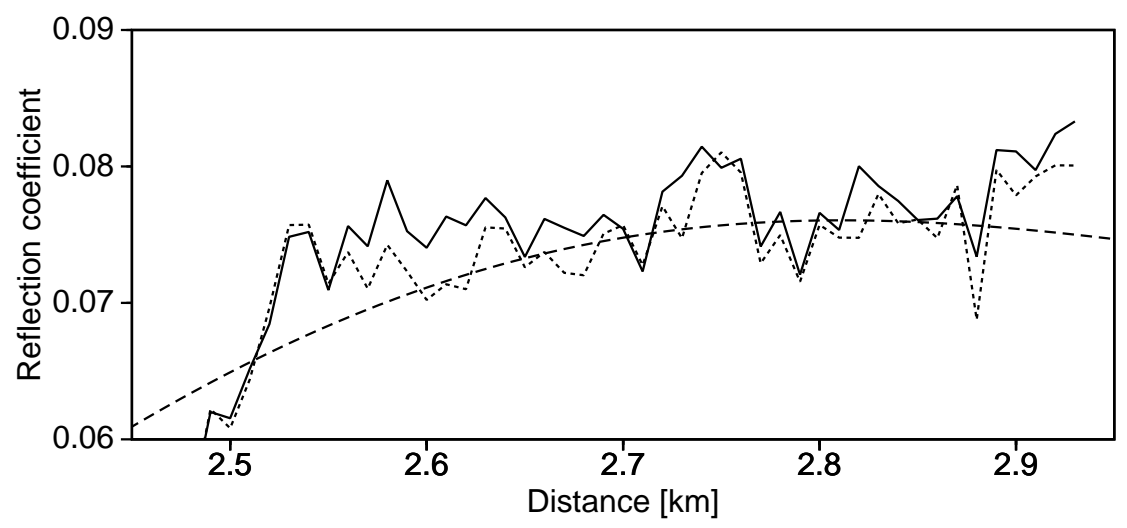

Figure 7.7: Results from noisy data for a $63^{\circ}$ inclined reflector: Dashed line: analytic reflection coefficients. Solid line: recovered reflection coefficients if the optimum aperture is used. Dotted line: recovered coefficients if the whole aperture is used.

Figure 7.4 shows that the AVO trend is preserved for all three cases, whether the whole aperture is used or the optimum aperture, with or without taper. For reflectors with moderate or no inclination the effect takes place on a smaller scale. Figure 7.6 shows that the results for a horizontal reflector (at $2.5 \mathrm{~km}$ depth and with the same velocities and wavelet as above) look virtually the same for both apertures.

The reflection coefficients that result from the noisy input data on the $63^{\circ}$ reflector are 

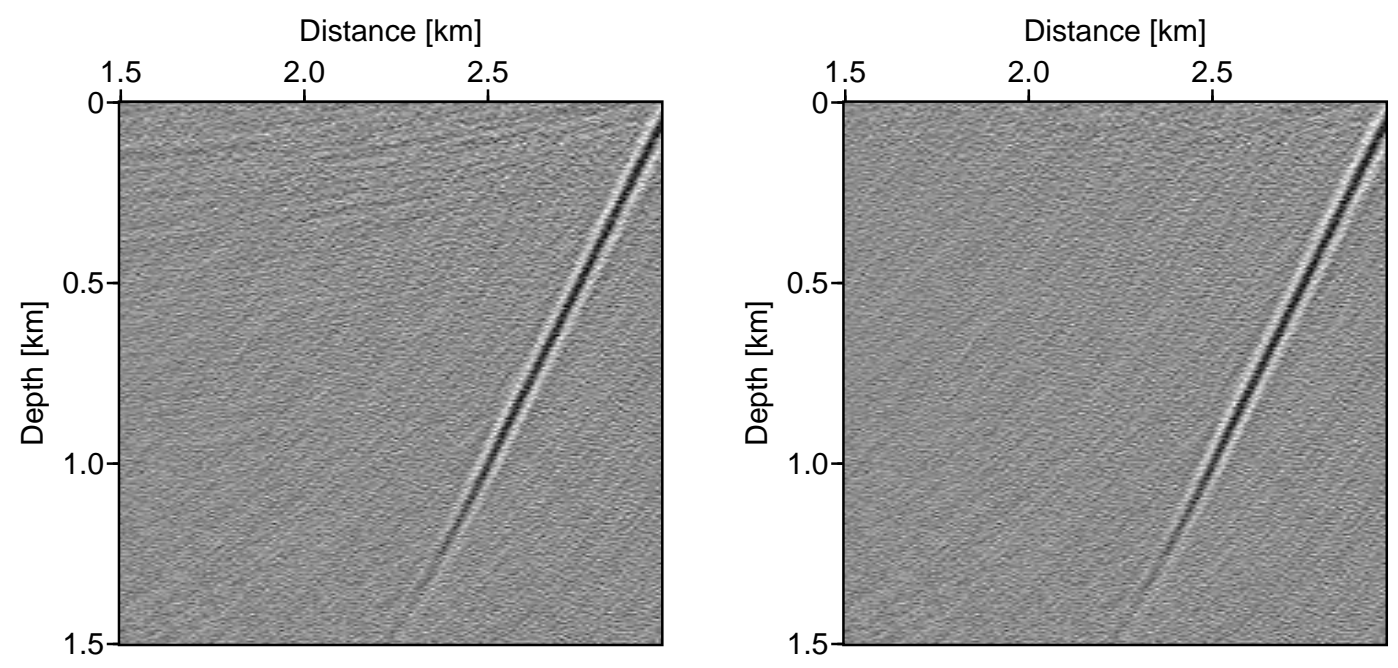

Figure 7.8: Migrated depth sections of noisy data for the $63^{\circ}$ inclined reflector. Left: the whole aperture was used; right: only traces within the optimum aperture were used.

shown in Figure 7.7 together with the analytic values. Please keep in mind that in this example the quality of the input section is very poor. For a better signal-to-noise ratio the scatter in the recovered reflection coefficients is significantly smaller. The migrated depth sections of the noisy data using the optimum aperture and the whole aperture are shown in Figure 7.8. In both cases the reflector has been migrated to the correct position but the image quality is improved if the optimum aperture is used. This effect may be more apparent if more than one arrival is present in the input section. If, e.g., another reflection event in the data would cut through the one under consideration, it would add unwanted contributions to the stack, thus increasing the noise. By limiting the aperture, this higher noise can at least be averted for unwanted events that lie outside of the aperture.

I have shown that application of the method to simple types of models yields good results in terms of image quality and recovered amplitude. The goal was to provide these good results and at the same time reduce the requirements in computational time and storage. The storage problem can be overcome by using coarse gridded traveltimes as only input data, as shown in Chapter 3 and 6 . The determination of the weight function from traveltimes as well as the interpolation of the diffraction time surfaces lead to savings in computational time, see also Chapter 3 and 6 . The following section discusses potential savings in computational time that result alone from the limited migration aperture. 


\subsection{Potential Savings in Computational Time}

The method of traveltime-based true-amplitude migration itself is very efficient in terms of computational time and storage, as already discussed in Chapter 3 and 6 . The savings in computational time that result from the reduction of the migration aperture alone are difficult to estimate because of the various factors involved in the size of the required aperture. To give an idea about possible savings, I have compared the optimum aperture to the complete aperture for the case of a single, horizontal reflector. The results are shown for 2-D and 3-D in Figure 7.9. Three parameters were varied to demonstrate their influence on the aperture: the velocity in the upper part of the model, the reflector depth, and the signal length. For small cable lengths, the savings are moderate because the required apertures are not much smaller than the total cable length. If the cable length is increased, the ratio of required aperture to complete aperture decreases. The effect of the velocity and signal length are similar since both are closely related to the wavelength. For varying reflector depth a more pronounced change occurs in the behaviour of the required aperture. The shallower the reflector is, the higher is the incidence angle of the wave with the normal to the registration surface, leading to an increase in apparent wavelength. This requires a larger aperture. 

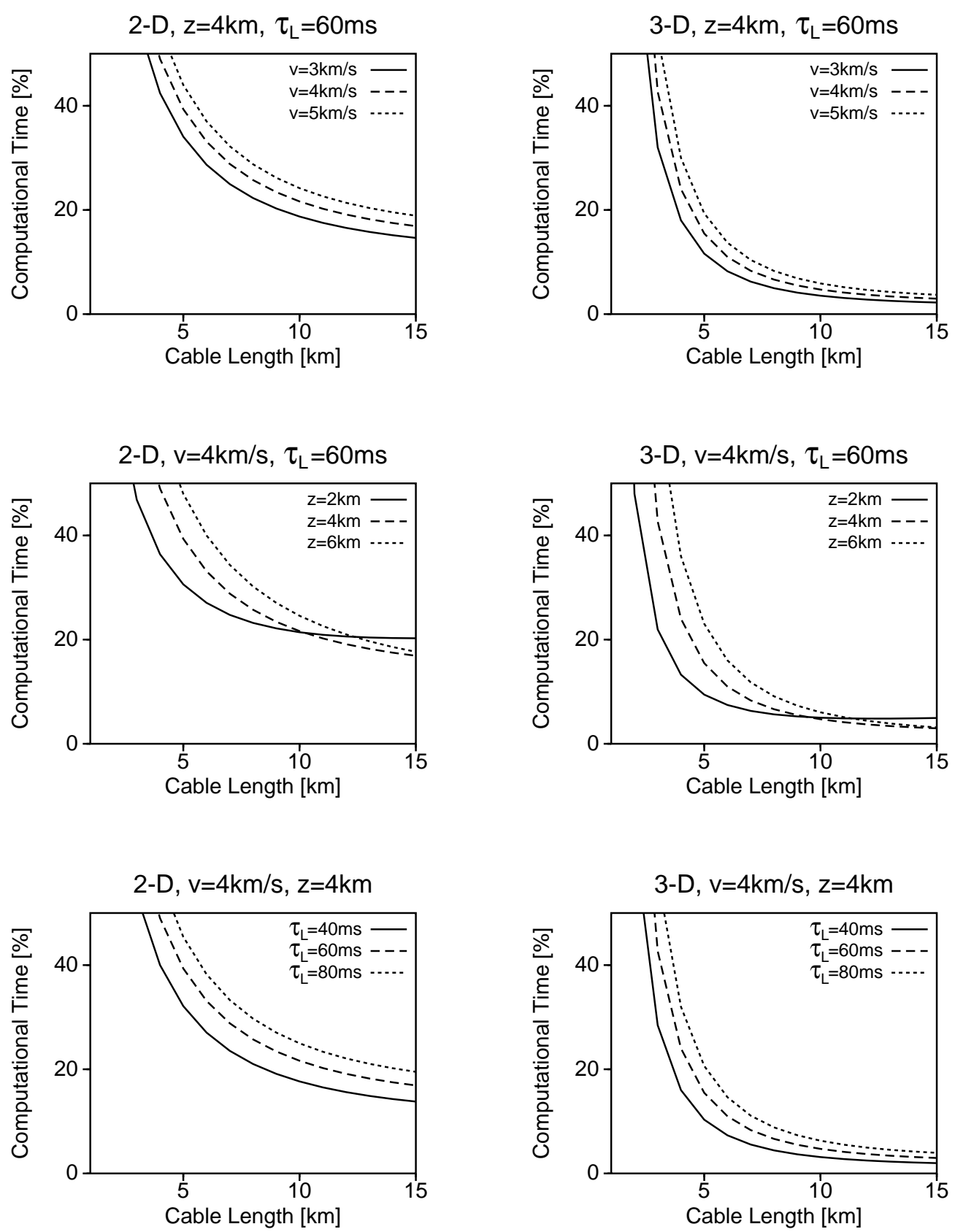

Figure 7.9: Potential savings in percent of computational time by using the optimum migration aperture (100\% corresponds to the complete aperture). The velocity model used here is a horizontal reflector. The parameters that were varied in these plots are the velocity in the upper layer (top), the reflector depth (middle) and the length of the source pulse (bottom), on the left for 2-D and right for 3-D. 


\section{Chapter 8}

\section{A General NMO Formula}

In the previous chapters I have considered applications based on traveltime tables as input information. These traveltimes were used to compute the first and second order spatial derivatives required for the applications. Another possibility not mentioned before is the determination of the coefficients directly from data. Reflection traveltimes may be obtained from seismic data by picking of events. Due to noise, however, picked traveltimes will probably not match the requirements for sufficiently accurate coefficients. In this chapter I will discuss an alternative for picking traveltimes. The coefficients can also be obtained by a stacking procedure and a subsequent semblance analysis, which allows a fully automated determination of the coefficients directly from seismic reflection data. This stacking procedure is called the common-reflection-surface (CRS) stack (Jäger et al., 2001; Zhang et al., 2001). It yields kinematic wavefield attributes that can be expressed by the traveltime coefficients used in this work and vice-versa.

Similar to NMO formulae, as, e.g, the classical $T^{2}-X^{2}$ equation, the CRS operator is a hyperbolic traveltime expression. Normal move-out (NMO) velocities play a significant role for the inversion of seismic reflection data. Closely related to the wavefront curvature, and, thus, geometrical spreading, NMO velocities are employed for a variety of important applications in seismic data processing, as, e.g., NMO corrections, divergence corrections, migration weights, traveltime interpolation, and determination of Fresnel zones. Similarly, the CRS attributes can be employed for the same applications. As I have shown in the previous chapters, the same properties can also be determined from the hyperbolic traveltime expression introduced in Chapter 3. The aim of this chapter is to show how equations like the CRS operator and the $T^{2}-X^{2}$ formula are related to the hyperbolic traveltime equation that can be considered as a general NMO formula.

In the following sections I will relate the classical $T^{2}-X^{2}$ formula to the hyperbolic traveltime expansion derived in Chapter 3. The general hyperbolic formula is also considered for the zero-offset and CMP configurations. The last section gives a brief introduction to the CRS technique and its operators, and how they relate to the general NMO equation. 


\subsection{Introduction to Classical Formulae}

The classical $T^{2}-X^{2}$ formula was derived for a horizontally stratified medium. Taner and Koehler (1969) showed that the reflection traveltime in the short spread approximation corresponds to a hyperbola of the form

$$
T^{2}=T_{0}^{2}+\frac{X^{2}}{V_{\mathrm{rms}}^{2}}
$$

In Equation (8.1.1) $V_{\text {rms }}$ is the RMS velocity controlling the move-out, $T_{0}$ is the vertical two way traveltime, and $X$ the distance from the source. The move-out in the $T^{2}-X^{2}$ domain is linear. It can be directly determined from the slope of the $T^{2}$ graph, which is, in the horizontal layer case, a straight line. A more general form of Equation (8.1.1),

$$
T^{2}=T_{0}^{2}+\frac{X^{2}}{V_{\text {nmo }}^{2}}
$$

contains the move-out velocity, $V_{\text {nmo }}$ instead of $V_{\text {rms }}$ (Hubral and Krey, 1980). In contrast to (8.1.1), Equation (8.1.2) is not restricted to models with horizontal layering. Hubral and Krey (1980) also show how the NMO velocity is related to the wavefront curvature.

In the next section I will introduce the general NMO equation and show that Equation (8.1.2) is a special case of it. To do this, the hyperbolic traveltime expansion (3.2.2) is considered in midpoint and half-offset coordinates.

\subsection{The General NMO Formula}

In Chapter 4 I have shown how the parabolic traveltime expansion, Equation (3.1.4), can also be applied to reflection traveltimes. Similarly, the hyperbolic traveltime expansion, Equation (3.2.2), can be rewritten for reflection traveltimes. Whereas the classical $T^{2}-X^{2}$ method was derived for horizontally stratified media, the hyperbolic traveltime expansion resulting from Equation (3.2.2) can be considered as an extension of the $T^{2}-X^{2}$ method to arbitrary 3 -D heterogeneous media. Since no assumptions were made on the model in the derivation of Equation (3.2.2), it even applies to anisotropic media.

In the following I assume that the registration surface in which sources and receivers are located coincides with the $x-y$ plane of the hyperbolic traveltime expansion given by (3.2.2). With the $\Delta z$ components of the source and receiver coordinates equal to zero, Equation (3.2.2) for the reflection traveltime becomes

$$
\tau^{2}(\boldsymbol{s}, \boldsymbol{g})=\left(\tau_{0}-\boldsymbol{p}_{0}^{\top} \Delta \boldsymbol{s}+\boldsymbol{q}_{0}^{\top} \Delta \boldsymbol{g}\right)^{2}+\tau_{0}\left(-2 \Delta \boldsymbol{s}^{\top} \underline{\mathrm{N}} \Delta \boldsymbol{g}-\Delta \boldsymbol{s}^{\top} \underline{\mathrm{S}} \Delta \boldsymbol{s}+\Delta \boldsymbol{g}^{\top} \underline{\mathrm{G}} \Delta \boldsymbol{g}\right) \quad .
$$

Vectors and matrices are defined in the recording surface and have dimension two. Equation (8.2.3) is valid in arbitrary, including anisotropic 3-D media. Regardless of the wave type it applies to monotypic reflections (PP, SS) as well as to converted waves (PS, SP). It is, therefore, a general hyperbolic move-out equation. 


\section{Midpoint and half-offset coordinates}

Equation (8.2.3) can be rewritten for the use of midpoint and half-offset coordinates. The midpoint coordinate is

$$
\boldsymbol{x}_{m}=\frac{\boldsymbol{g}+\boldsymbol{s}}{2}
$$

and the half-offset coordinate is

$$
\boldsymbol{h}=\frac{\boldsymbol{g}-\boldsymbol{s}}{2}
$$

Substituting these into (8.2.3) leads to

$$
\begin{aligned}
\tau^{2}= & \left(\tau_{0}+\left(\boldsymbol{q}_{0}-\boldsymbol{p}_{0}\right)^{\top} \Delta \boldsymbol{x}_{m}+\left(\boldsymbol{q}_{0}+\boldsymbol{p}_{0}\right)^{\top} \Delta \boldsymbol{h}\right)^{2}+2 \tau_{0} \Delta \boldsymbol{h}^{\top}\left(\underline{\mathrm{G}}+\underline{\mathrm{S}}-2\left(\underline{\mathrm{N}}^{\top}-\underline{\mathrm{N}}\right)\right) \Delta \boldsymbol{x}_{m} \\
& +\tau_{0} \Delta \boldsymbol{x}_{m}^{\top}(\underline{\mathrm{G}}-\underline{\mathrm{S}}-2 \underline{\mathrm{N}}) \Delta \boldsymbol{x}_{m}+\tau_{0} \Delta \boldsymbol{h}^{\top}(\underline{\mathrm{G}}-\underline{\mathrm{S}}+2 \underline{\mathrm{N}}) \Delta \boldsymbol{h} .
\end{aligned}
$$

Equation (8.2.6) will now be investigated for the CMP and zero-offset configurations.

\section{Arbitrary medium in CMP configuration}

For the CMP configuration $\Delta \boldsymbol{s}=-\Delta \boldsymbol{g}$. The CMP coordinates for a 3-D medium are the offset $r$ and the azimuth angle $\varphi$. They relate to the vectors $\Delta \boldsymbol{s}$ and $\Delta \boldsymbol{g}$ as follows:

$$
\begin{aligned}
& -\Delta s_{x}=\Delta g_{x}=\frac{r}{2} \cos \varphi \\
& -\Delta s_{y}=\Delta g_{y}=\frac{r}{2} \sin \varphi .
\end{aligned}
$$

Substituting these into the hyperbolic traveltime expansion and introducing the abbreviations

$$
\begin{aligned}
P= & \frac{1}{2}\left[\left(p_{x}+q_{x}\right) \cos \varphi+\left(p_{y}+q_{y}\right) \sin \varphi\right] \\
N= & \frac{1}{4}\left[\left(G_{x x}-S_{x x}+2 N_{x x}\right) \cos ^{2} \varphi+\left(G_{y y}-S_{y y}+2 N_{y y}\right) \sin ^{2} \varphi\right. \\
& \left.+2\left(G_{x y}-S_{x y}+N_{x y}+N_{y x}\right) \sin \varphi \cos \varphi\right]
\end{aligned}
$$

leads to

$$
\begin{aligned}
\tau^{2} & =\left(\tau_{0}+P r\right)^{2}+\tau_{0} N r^{2} \\
& =\tau_{0}^{2}+2 \tau_{0} \operatorname{Pr}+\left(P^{2}+\tau_{0} N\right) r^{2}
\end{aligned}
$$

This equation is generally valid for the CMP configuration, regardless of the model under consideration. It is even valid for converted waves. It is the equation of a shifted hyperbola. 


\section{Zero-offset situation for a monotypic wave}

Here the hyperbolic equation must be symmetric with respect to exchange of $\Delta \boldsymbol{s}$ and $\Delta \boldsymbol{g}$. This requires that

$$
\begin{aligned}
\boldsymbol{q}_{0} & =-\boldsymbol{p}_{0} \\
\underline{\mathrm{G}} & =-\underline{\mathrm{S}} \\
\underline{\mathrm{N}} & =\underline{\mathrm{N}}^{\top} .
\end{aligned}
$$

Therefore equation (8.2.3) reduces to

$$
\tau^{2}=\left(\tau_{0}+\boldsymbol{q}_{0}^{\top}(\Delta \boldsymbol{s}+\Delta \boldsymbol{g})\right)^{2}+\tau_{0}\left((\Delta \boldsymbol{s}+\Delta \boldsymbol{g})^{\top} \underline{\mathrm{G}}(\Delta \boldsymbol{s}+\Delta \boldsymbol{g})-2 \Delta \boldsymbol{s}^{\top}(\underline{\mathrm{G}}+\underline{\mathrm{N}}) \Delta \boldsymbol{g}\right)
$$

It can also be expressed in midpoint and half-offset coordinates:

$$
\tau^{2}=\left[\tau_{0}+2 \boldsymbol{q}_{0}^{\top} \Delta \boldsymbol{x}_{m}\right]^{2}+2 \tau_{0}\left[\Delta \boldsymbol{x}_{m}^{\top}(\underline{\mathrm{G}}-\underline{\mathrm{N}}) \Delta \boldsymbol{x}_{m}+\Delta \boldsymbol{h}^{\top}(\underline{\mathrm{G}}+\underline{\mathrm{N}}) \Delta \boldsymbol{h}\right] .
$$

\section{Zero-offset CMP configuration}

Using again the symmetric properties for zero-offset and monotypic waves given by (8.2.11), Equation (8.2.10) can be simplified to

$$
\tau^{2}=\tau_{0}^{2}+\tau_{0} N r^{2}
$$

where $N$ is

$$
N=\frac{1}{2}\left[\left(G_{x x}+N_{x x}\right) \cos ^{2} \varphi+\left(G_{y y}+N_{y y}\right) \sin ^{2} \varphi+2\left(G_{x y}+N_{x y}\right) \sin \varphi \cos \varphi\right] .
$$

Comparing equation (8.2.14) to the NMO equation (8.1.2) rewritten to midpoint and half-offset coordinates

$$
\tau^{2}=\tau_{0}^{2}+\frac{r^{2}}{v_{\mathrm{nmo}}^{2}}
$$

leads to an expression for the NMO velocity in terms of second order traveltime derivatives:

$$
v_{\mathrm{nmo}}^{2}=\frac{1}{\tau_{0} N}
$$

For a $v(z)$ (homogeneously layered) medium $N$ can be further simplified: in this case the matrices $\underline{\mathrm{G}}$ and $\underline{\mathrm{N}}$ are identical. The mixed components $N_{x y}$ vanish and $N_{x x}=N_{y y}=N$.

\section{Examples for the zero-offset CMP configuration}

For a homogeneous medium with one horizontal reflector at the depth $z_{0}$ the traveltime is

$$
\tau=\frac{1}{v} \sqrt{\left(g_{x}-s_{x}\right)^{2}+\left(g_{y}-s_{y}\right)^{2}+4 z_{0}^{2}} .
$$


The second order derivatives are given by

$$
N=\frac{1}{2 v z_{0}}
$$

The $\tau_{0}$ time is the two-way-traveltime

$$
\tau_{0}=\frac{2 z_{0}}{v}
$$

From this follows equation (8.2.17) with $v_{\text {nmo }}=v$. For a layered medium above the reflector the NMO velocity becomes $v_{\text {rms }}$. This corresponds to Equation (8.1.1), the $T^{2}-X^{2}$ equation.

For a 2-D homogeneous model with an inclined reflector (inclination angle $\vartheta$ ) and $s_{x}$ and $g_{x}$ the distances of the source and receiver to the point where the reflector intersects the $x$-surface, the traveltime is

$$
\tau=\frac{1}{v} \sqrt{\left(g_{x}-s_{x}\right)^{2}+4 g_{x} s_{x} \sin ^{2} \vartheta} .
$$

The second derivative $N=N_{x x}$ for $s_{x}=g_{x}=x$ is

$$
N=\frac{1}{2 v x} \frac{\cos ^{2} \vartheta}{\sin \vartheta}
$$

and furthermore with

$$
\tau_{0}=\frac{2 x \sin \vartheta}{v}
$$

the NMO velocity is

$$
v_{\mathrm{nmo}}=\frac{v}{\cos \vartheta}
$$

\subsection{The CRS Formula}

The Common-Reflection-Surface (CRS) stack provides a 2-D simulated common-offset section from seismic multi-coverage reflection data. In contrast to conventional reflection imaging like NMO-DMO processing or depth migration the CRS stack does not depend on a macro-velocity model. The output from the CRS stack not only provides a wellsimulated common-offset section - including the zero-offset case - but also five kinematic wavefield attribute sections which can be used to construct a macro-velocity model (Jäger et al., 2001), compute geometrical spreading, and to distinguish between reflection events and diffractions (Zhang et al., 2001).

\section{Zero-Offset CRS Formula}

The zero-offset CRS formula (see, e.g., Jäger et al. 2001) is a hyperbolic three-parameter traveltime expression in midpoint and half-offset coordinates. It is closely related to the Polystack (deBazelaire, 1988) and the multi-focusing operator (Gelchinsky et al., 1999). 
Since the CRS stacking surface provides a better fit to the reflection events than the stacking trajectories in conventional stacking methods, the resulting simulated zero-offset section is of high quality. The three kinematic attributes for the zero-offset CRS formula are

- the emergence angle of the zero-offset ray, $\alpha_{0}$,

- the curvature of the emerging normal-incident-point (NIP) wave, $K_{\mathrm{NIP}}$, a wavefront that corresponds to a wave emitted from a point source at the normal-incidencepoint,

- the curvature of the emerging normal $(\mathrm{N})$ wave, $K_{\mathrm{N}}$, a wavefront that corresponds to a wave generated by an exploding reflector.

For more details on N and NIP waves, please refer to Hubral (1983). The zero-offset CRS formula is given by (Jäger et al., 2001)

$$
\tau_{\mathrm{ZO}-\mathrm{CRS}}^{2}=\left[\tau_{0}+\frac{2 \sin \alpha_{0}}{v_{0}} \Delta x_{m}\right]^{2}+\frac{2 \tau_{0} \cos ^{2} \alpha_{0}}{v_{0}}\left[K_{\mathrm{N}} \Delta x_{m}^{2}+K_{\mathrm{NIP}} \Delta h^{2}\right],
$$

where the velocity $v_{0}$ at the zero-offset position is assumed to be known from a priori information.

Equation (8.3.25) is equivalent to the 2-D variant of Equation (8.2.13),

$$
\tau^{2}=\left(\tau_{0}+2 q_{0} \Delta x_{m}\right)^{2}+2 \tau_{0}\left[(G-N) \Delta x_{m}^{2}+(G+N) \Delta h^{2}\right],
$$

with $q_{0}=\sin \alpha_{0} / v_{0}$ and

$$
\begin{aligned}
K_{\mathrm{N}} & =\frac{v_{0}}{\cos ^{2} \alpha_{0}}(G-N) & N=\frac{\cos ^{2} \alpha_{0}}{2 v_{0}}\left(K_{\mathrm{NIP}}-K_{\mathrm{N}}\right) \\
K_{\mathrm{NIP}} & =\frac{v_{0}}{\cos ^{2} \alpha_{0}}(G+N) & G=\frac{\cos ^{2} \alpha_{0}}{2 v_{0}}\left(K_{\mathrm{NIP}}+K_{\mathrm{N}}\right) .
\end{aligned}
$$

\section{Common-Offset CRS Formula}

The common-offset CRS formula is an extension of Equation (8.3.25) which yields a simulated common-offset section (Zhang et al., 2001). It uses the following five kinematic attributes

- the emergence angle at the source, $\alpha_{s}$,

- the incidence angle at the receiver, $\alpha_{g}$,

- the wavefront curvature $K_{\mathrm{CS}}^{\mathrm{G}}$ of a wave generated by a point source at the source position and measured at the receiver position, corresponding to a common-shot experiment,

- the wavefront curvature $K_{\mathrm{CMP}}^{\mathrm{G}}$ of a wave from a fictitious CMP experiment, measured at the receiver, 
- the wavefront curvature $K_{\mathrm{CMP}}^{\mathrm{S}}$ of a wave from a fictitious CMP experiment, measured at the source.

The common-offset CRS formula is given by (Zhang et al., 2001)

$$
\begin{aligned}
\tau_{\mathrm{CO}-\mathrm{CRS}}^{2}= & {\left[\tau_{0}+\left(\frac{\sin \alpha_{g}}{v_{g}}-\frac{\sin \alpha_{s}}{v_{s}}\right) \Delta x_{m}+\left(\frac{\sin \alpha_{g}}{v_{g}}+\frac{\sin \alpha_{s}}{v_{s}}\right) \Delta h\right]^{2} } \\
& +\tau_{0}\left[\left(4 K_{\mathrm{CS}}^{\mathrm{G}}-3 K_{\mathrm{CMP}}^{\mathrm{G}}\right) \frac{\cos ^{2} \alpha_{g}}{v_{g}}-K_{\mathrm{CMP}}^{\mathrm{S}} \frac{\cos ^{2} \alpha_{s}}{v_{s}}\right] \Delta x_{m}^{2} \\
& +\tau_{0}\left[K_{\mathrm{CMP}}^{\mathrm{G}} \frac{\cos ^{2} \alpha_{g}}{v_{g}}-K_{\mathrm{CMP}}^{\mathrm{S}} \frac{\cos ^{2} \alpha_{s}}{v_{s}}\right] \Delta h^{2} \\
& +2 \tau_{0}\left[K_{\mathrm{CMP}}^{\mathrm{G}} \frac{\cos ^{2} \alpha_{g}}{v_{g}}+K_{\mathrm{CMP}}^{\mathrm{S}} \frac{\cos ^{2} \alpha_{s}}{v_{s}}\right] \Delta h \Delta x_{m}
\end{aligned}
$$

where the velocities $v_{g}$ and $v_{s}$ are again assumed to be known from a priori information.

Equation (8.3.28) is equivalent to the 2-D variant of Equation (8.2.6),

$$
\begin{aligned}
\tau^{2}= & \left(\tau_{0}+\left(q_{0}-p_{0}\right) \Delta x_{m}+\left(q_{0}+p_{0}\right) \Delta h\right)^{2}+2 \tau_{0}(G+S) \Delta x_{m} \Delta h \\
& +\tau_{0}\left[(G-S-2 N) \Delta x_{m}^{2}+(G-S+2 N) \Delta h^{2}\right]
\end{aligned}
$$

with $q_{0}=\sin \alpha_{g} / v_{g}, p_{0}=\sin \alpha_{s} / v_{s}$, and

$$
\begin{aligned}
K_{\mathrm{CMP}}^{\mathrm{S}} & =\frac{v_{s}}{\cos ^{2} \alpha_{s}}(S-N) \\
K_{\mathrm{CMP}}^{\mathrm{G}} & =\frac{v_{g}}{\cos ^{2} \alpha_{g}}(G+N) \\
K_{\mathrm{CS}}^{\mathrm{G}} & =\frac{v_{g}}{\cos ^{2} \alpha_{g}} G .
\end{aligned}
$$

The inverse relations between $G, N, S$ and $K_{\mathrm{CMP}}^{\mathrm{S}}, K_{\mathrm{CMP}}^{\mathrm{G}}$, and $K_{\mathrm{CS}}^{\mathrm{G}}$ are

$$
\begin{aligned}
G & =\frac{\cos ^{2} \alpha_{g}}{v_{g}} K_{\mathrm{CS}}^{\mathrm{G}} \\
N & =\frac{\cos ^{2} \alpha_{g}}{v_{g}}\left(K_{\mathrm{CMP}}^{\mathrm{G}}-K_{\mathrm{CS}}^{\mathrm{G}}\right) \\
S & =\frac{\cos ^{2} \alpha_{s}}{v_{s}} K_{\mathrm{CMP}}^{\mathrm{S}}+\frac{\cos ^{2} \alpha_{g}}{v_{g}}\left(K_{\mathrm{CMP}}^{\mathrm{G}}-K_{\mathrm{CS}}^{\mathrm{G}}\right) .
\end{aligned}
$$

Of course, the common-offset CRS formula, Equation (8.3.28) is also applicable to the zero-offset situation, where $q_{0}=-p_{0}$ and $G=-S$, see (8.2.11). The relationships between the CRS attributes are in this case given by

$$
\begin{aligned}
\alpha_{g} & =-\alpha_{s}=\alpha_{0} \\
K_{\mathrm{CS}}^{\mathrm{G}} & =\frac{K_{\mathrm{NIP}}+K_{\mathrm{N}}}{2} \\
K_{\mathrm{CMP}}^{\mathrm{G}} & =-K_{\mathrm{CMP}}^{\mathrm{S}}=K_{\mathrm{NIP}} .
\end{aligned}
$$




\section{Chapter 9}

\section{Conclusions}

I have presented an efficient strategy for amplitude-preserving migration of the Kirchhoff type. It is based on coarse gridded traveltimes as only input information and therefore requires a small amount of computer storage. The traveltimes are employed for the computation of all quantities required for the migration process, namely fine gridded traveltimes for the stacking surface, the true-amplitude weight functions, and the size of the optimum migration aperture. The method also provides a tool for the computation of geometrical spreading, and move-out velocities.

The technique for the interpolation of traveltimes is based on the traveltime differences (i.e., move-out) between neighbouring sources and receivers of a multi-fold experiment. All coefficients for the interpolation are computed from traveltimes on a coarse grid. The interpolation has a high accuracy since it acknowledges the curvature of the wavefront and is thus exact up to the second order. Following from the investigation of the accuracy of the coefficients I recommend the hyperbolic rather than parabolic traveltime expansion. This commendation is supported by the generic examples. Both hyperbolic and parabolic variants are, however, far superior to the often used trilinear interpolation. The difference in computational time for the three variants is insignificant.

One important feature of the technique is its possibility to interpolate between sources, not only between receivers. The fact that all necessary coefficients can be computed on a coarse grid leads to considerable savings in computational time and computer memory since traveltime tables for less sources need to be generated as well as kept in storage. If, e.g., every tenth grid point in three dimensions is used, this corresponds to a factor of $10^{5}$ (if the sources are located in the $x-y$ surface) less in storage requirement for interpolation of shots and receivers at no significant loss in accuracy. As the method is not restricted to cubical grids the coarse grid spacing can be adapted to the model under consideration.

Since the matrices involved in the traveltime interpolation bear a close relationship to the matrices employed in the paraxial ray approximation the technique can also be used to determine dynamic wavefield properties. This leads to the determination of the complete ray propagator just from traveltimes. The ray propagator can be used for various tasks including the computation of geometrical spreading. As for the traveltime 
interpolation, the coefficients from the hyperbolic traveltime expansion are better suited for the computation of geometrical spreading, which is confirmed by several examples.

The same coefficients as for the traveltime interpolation were also applied for the determination of migration weight functions. The examples given show good accordance between the reconstructed reflectors and theoretical values in terms of position as well as in amplitudes. This applies to PP as well as to PS converted wave data. A further significant reduction in computational time was achieved by limiting the required migration aperture to a minimum. A simple example indicates potential savings of up to 80 percent in $2-\mathrm{D}$ and more than 90 percent in $3-\mathrm{D}$. The size of the optimum aperture was also computed from the traveltime coefficients. Moreover, use of a limited aperture can enhance the image quality by reducing migration noise.

I have presented simple examples for the true-amplitude migration in order to compare the reconstructed values to analytic reflection coefficients. The high accuracy of the traveltime interpolation and traveltime-based computation of geometrical spreading for the Marmousi example supports my assumption that the method will also perform well in complex models.

Finally, I have shown that the hyperbolic reflection traveltime formula can be considered as an extension of the $T^{2}-X^{2}$ method to arbitrary 3-D media, and how this general NMO equation is related to other move-out formulae.

Since no assumptions on the model were made when deriving the governing equations of this research, there is a so far unused potential to extend this technique to anisotropic media. The computational advantages of the traveltime-based method will be even better for these media. Suggestions concerning this extension of the method are outlined in the following "Outlook" chapter. 


\section{Chapter 10}

\section{Outlook}

This thesis has introduced the technique of traveltime-based true-amplitude migration in isotropic media. Application of the method to numerical examples illustrates that the method promises to become a powerful tool in image processing. There remain, however, aspects which are worth a thorough investigation. Some were already mentioned, for example, the application of the method to complex models and associated problems. These problems are closely related to another topic of fundamental interest, which has, so far not been systematically researched: the influence of errors in the velocity model on the recovered amplitudes. The first section of this chapter considers this influence. Since, especially in complex models, the uniform illumination of the subsurface point under consideration is crucial, a migration implementation allowing for this would enhance the quality of the resulting image and amplitudes. It could be achieved by migration with angular parametrisation, which is described in the next section.

Another major issue for the future will be the extension of the method to anisotropic media. Anisotropy has been recognised to be important during the last years. An efficient implementation of anisotropic amplitude-preserving migration would be of considerable interest, the more, since there appear to be only very few studies on the concept of amplitude-preserving migration in anisotropic media. The last section gives some thought to how this could be achieved.

\subsection{Sensitivity to Velocity Errors}

For carrying out a Kirchhoff depth migration, an initial velocity model is required. Since this thesis was not concerned about model-building, it was so far assumed that a velocity model already exists, for instance as the result of a previous migration step, and that this velocity model is correct. This last assumption is, of course, not justified. The velocity model enters the migration in various ways. Errors in the velocity model cause wrong traveltimes. These show in the migrated (kinematic) image in terms of under- and over-migration (i.e. smiles and frowns). However, even horizontal alignment of events in a common image gather does not signify that the velocity model which was used for the migration is correct (Menyoli and Gajewski, 2001). Therefore, one must always assume that the velocity model under consideration has errors. The traveltime errors 
which result from the wrong velocities also cause errors in the true-amplitude migration weight functions, and, therefore in the recovered amplitudes. Since this is always the case, the correlation between the errors in the velocity model and those of the recovered amplitudes should be investigated.

For one special case of velocity deviations, namely smoothing of velocity models, I have already shown in a simple example how this leads to errors in the amplitudes (Section 6.8). The effect of smoothing has also been recognised by Peles et al. (2001). A more general approach for simple types of media, where an analytic solution is available, would be to express the traveltime error $\Delta \tau$ in terms of the velocity error $\Delta v$ by

$$
\Delta \tau=\frac{\partial \tau}{\partial v} \Delta v
$$

Subsequent differentiation of Equation (10.1.1) will then lead to expressions for the error of those traveltime derivatives which are involved in the weight functions. Since the main contribution to the stack comes from traces in the vicinity of the stationary ray, the deviation of the weights for these traces will give insight to the effect that velocity errors have on the recovered amplitudes.

To investigate the amplitude errors in arbitrary isotropic media, the generalised NMOequation (8.2.14) can be employed:

$$
\tau^{2}=\tau_{0}^{2}+\frac{r^{2}}{v_{\mathrm{nmo}}^{2}}=\tau_{0}^{2}+\tau_{0} N r^{2}
$$

This equation connects the traveltime $\tau$ to the NMO velocity $v_{\text {nmo. }}$. The quantity $N$ contains second order derivatives which enter into the weight function. Therefore Equation (10.1.2) can be used to describe errors of $N$ in terms of traveltime errors. For arbitrary media the traveltime errors can be found by perturbation methods. The deviation of the traveltime $\tau$ of the traveltime of the unperturbed medium $\tau_{\text {ref }}$ is given by

$$
\Delta \tau=\tau-\tau_{\text {ref }}=-\int_{s_{0}}^{s} \frac{\Delta v}{v^{2}} \mathrm{~d} s
$$

where $v$ is the velocity of the unperturbed medium and $\Delta v$ the deviation of it. The integration is carried out along the ray path of the unperturbed medium. As before, differentiation of Equation (10.1.3) leads to expression for the errors of the traveltime derivatives that are needed for the weight functions.

A similar approach can be followed for $\mathrm{qP}$ waves in weakly anisotropic media. Jech and Pšenčík (1989) give the traveltime error by

$$
\Delta \tau=-\frac{1}{2} \int_{s_{0}}^{s} \frac{\Delta a_{i j k l} p_{i} p_{l} g_{j} g_{k}}{v} \mathrm{~d} s
$$


where the $\Delta a_{i j k l}$ are the deviations of the density-normalised elastic parameters from those of the unperturbed medium, $p_{i}$ are the slownesses and $g_{j}$ the polarisation vectors. The following procedure is the same as for isotropic media. It requires, however, that expressions for the true-amplitude weight functions exist for anisotropic media. This point will be discussed below, in section 10.3.

It may even be possible that the results of these investigations lead to criteria for a better focusing. If this would be the case, the results may be used to formulate a migration-based modeling technique in addition to horizontal image gathers. To predict such a method at this time, however, would be premature.

\subsection{Migration with Angular Parametrisation}

In a typical seismic experiment, the sources and receivers are spaced as uniformly as possible in the recording surface. This line-up has, however, a vital disadvantage: the even coverage at the registration surface does not coincide with equable illumination at the image point, which is necessary for AVO studies. To achieve uniform illumination at the image point, rays with equal angular distance are required from the image point to the registration surface. Rays which leave the image point with equi-angular spacing, however, do not arrive with equidistant spacing at the recording surface. Therefore equidistant spacing of sources and receivers leads to high illumination in some angular regions, and poor illumination in others. Figure 10.1 (left) illustrates this effect which is already present in homogeneous media. It has even higher impact in complex media, e.g., with shadow zones.
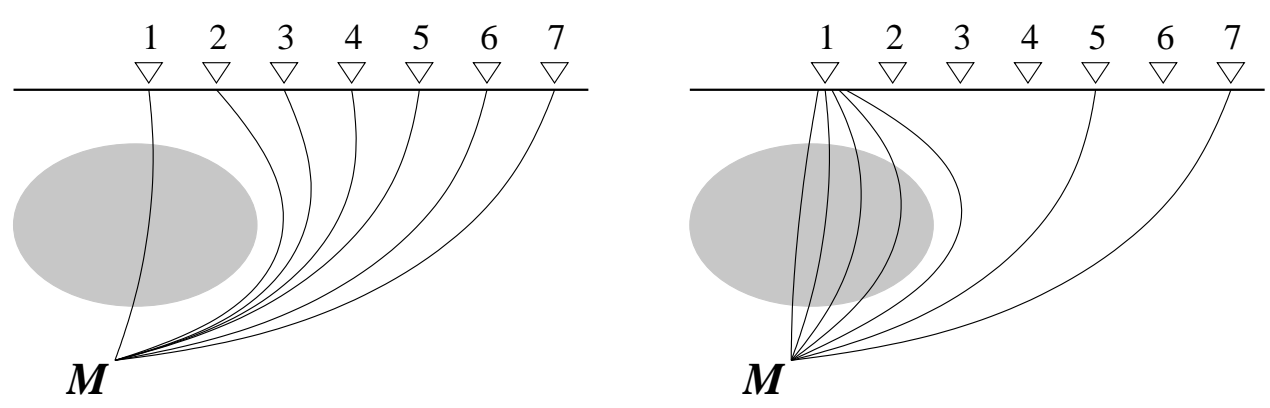

Figure 10.1: Schematic ray paths for a medium with a low velocity zone (gray) from an image point $M$ to several receivers (triangles). Left: in conventional migration only a small angular region is well illuminated at $M$. On the right, the angles of the rays starting from $M$ are evenly distributed. This leads to even illumination at the image point, which is especially important for shadow zones (see receivers $2-4$ ).

To provide AVO curves, the illumination of the image point should be evenly distributed over a large angular area. Since a model dependent registration can not be realised, a specific implementation of migration could compensate this shortfall: the migration with angular parametrisation, which was suggested by Brandsberg-Dahl et al. (2001). 
In this implementation, the traces in the registration surface are chosen to span a large angle at the image point as evenly as possible (see Figure 10.1, right). This is achieved by specifying the emergence angle, respectively the slowness at the image point. At the same time this procedure avoids the problematic triplications, because a ray is non-ambiguously characterised by its slowness. Therefore it may be convenient to implement this algorithm using traveltimes for sources in the subsurface, i.e. at the image points. For such an implementation the hyperbolic traveltime interpolation would be very useful since it allows to interpolate sources.

Although the migration with angular parametrisation has already been proposed by Brandsberg-Dahl et al. (2001), an implementation based on the hyperbolic traveltime interpolation will be also especially suited, because then, the uniform coverage at the image point will be particularly easy to realize. The slowness of a ray from $M=\boldsymbol{s}_{0}$ to a receiver at $\boldsymbol{g}_{0}+\Delta \boldsymbol{g}$ is given by

$$
\boldsymbol{p}=\boldsymbol{p}_{0}+\tau_{0} \underline{\mathrm{N}} \Delta \boldsymbol{g}
$$

The quantities $\tau_{0}, \boldsymbol{p}_{0}$, and $\underline{\mathrm{N}}$ correspond to the traveltime and its derivatives for a ray from $M$ to $\boldsymbol{g}_{0}$. The emergence angle at $M$ is given by the slowness $\boldsymbol{p}$ at $M$. Therefore, Equation (10.2.5) leads to the receiver position $\boldsymbol{g}_{0}+\Delta \boldsymbol{g}$ for a given emergence angle. Since in most cases there will be no receiver at this position, trace interpolation is required, which is discussed, e.g., in Spitz (1991) or Kabir and Verschuur (1995).

\subsection{Anisotropy}

So far, amplitude-preserving migration has almost exclusively been applied to PP data, where an isotropic medium was assumed. Although anisotropy is recognised as important it is only considered with respect to kinematic aspects but not to amplitudes. Even the kinematics are in most cases reduced to simple types of media, e.g., media with transverse isotropy. If we are dealing with an anisotropic earth, however, the application of isotropic methods leads to problems. Anisotropy can have several causes, as for example intrinsic anisotropy or layer-induced anisotropy. Other possibilities are oriented fluid-filled crack systems, and, of course, a combination of these causes, leading to an effective anisotropy. In summary, as soon as there exists an organised structure with a preferred orientation, anisotropy shows, if the wavelength of the investigation method is larger than the scale of the structure. This problem of different scales has practical relevance, if results from measurements on different scales are to be combined, e.g., surface and bore hole seismics. If anisotropy is not considered, these results will not coincide (i.e. leading to mis-ties etc.).

Anisotropic effects are not restricted to kinematics. They have considerable influence on the AVO- or AVA-behaviour of geological interfaces. One example are shales and sands with a similar acoustic impedance. The anisotropy of the shales can lead to a reversal in the polarity of the reflection coefficient that does not occur for isotropy. Another possibility are amplitude changes assigned to the presence of gas if a medium is assumed to be isotropic. In an anisotropic medium, this behaviour can be explained without gas (deHoop et al., 1999). Therefore, anisotropy must also be acknowledged 
where amplitudes are considered.

A theoretical representation of Kirchhoff migration in anisotropic media was given by deHoop and Bleistein (1997). This very complex theory is not very well suited for an implementation. Although Gerea et al. (2000) give an anisotropic example for amplitude-preserving migration of multi-component data, they use a simplified weight function and consider only a horizontally layered VTI medium. This approach is very restricted and will not be applicable to media with more complex anisotropy. The author is not aware of additional work on this topic.

The first step in extending the method of traveltime-based true-amplitude migration to anisotropic media is again the traveltime interpolation. It is even more important to have an efficient implementation for the computation of the diffraction time surface than for isotropic media, because the computational effort for anisotropic traveltime generation is about five times higher than for isotropic media (D. Gajewski, personal communication). Since no assumptions on the model were made for the derivation of the hyperbolic traveltime expansion, Equation (3.2.2), it is equally valid for anisotropic media. If traveltimes for suitable source-receiver combinations are available, all coefficients in Equation (3.2.2) can again be determined from (coarse-gridded) traveltimes.

In the anisotropic case, however, the expressions (3.1.7), and (3.1.10) to (3.1.15) for the derivatives with respect to the $z$-component of the source positions are not valid anymore. These relations were determined by considering the isotropic eikonal equation (3.1.6) and they contain spatial derivatives of the phase velocity. In anisotropic media, the phase velocity depends not only on the position, but also on the direction of the ray. Gajewski (1993) introduces derivatives of the phase velocity with respect to the ray direction. Following his approach could lead to according expressions, but with respect to the $x$-, $y$-, and $z$-coordinates. Note that these derivatives are only required for the traveltime interpolation, if the position of the source changes in depth. All coefficients are, however, necessary for the determination of geometrical spreading, migration weights, and limited aperture migration. At this time, their determination is possible, but requires traveltime tables for additional source positions at different depths (see Appendix D).

Figure 10.2 shows results of a hyperbolic traveltime interpolation in a homogeneous medium with elliptical anisotropy. The velocities which were used for this example are $v_{x}=4 \mathrm{~km} / \mathrm{s}$, and $v_{z}=5 \mathrm{~km} / \mathrm{s}$ (see Appendix C). Input traveltimes were given by the analytic solution (see also Appendix C) on a $100 \mathrm{~m}$ grid. Hyperbolic coefficients were determined and used for subsequent interpolation onto a fine $10 \mathrm{~m}$ grid, first without a change in source position (Figure 10.2, left), and then for a source which was shifted by $50 \mathrm{~m}$ in $x$ and $y$ (Figure 10.2, right). Since the hyperbolic approximation equals the exact solution in this case, the errors are caused by the limited machine precision: for both source positions their median is below $7 \times 10^{-6 \%}$. The maximum value is smaller than $10^{-4 \%}$ apart for the source region, where the division by $\tau_{0}=0$ leads to wrong coefficients.

To find expressions for the weight functions, the same approach as for isotropic media may 

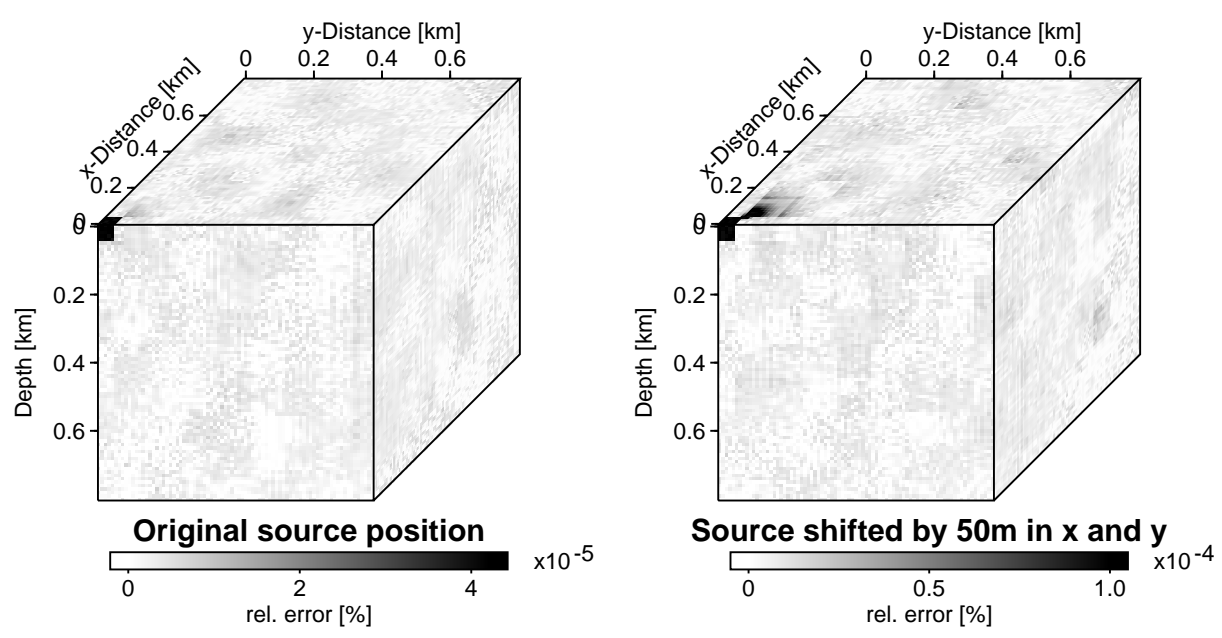

Figure 10.2: Traveltime errors for a homogeneous velocity model with elliptical anisotropy. Left: traveltimes were interpolated from a $100 \mathrm{~m}$ coarse grid onto a $10 \mathrm{~m}$ fine grid without a change of the source position. Right: as before, but additionally, the source was shifted $50 \mathrm{~m}$ in $x$ - and $y$-direction. The errors are within machine precision apart from the near source vicinity. In this region, the hyperbolic coefficients are wrong, because of the division by $\tau_{0}$, which is zero at the source.

be used. In the derivations of the isotropic weight functions in Chapter 6 no assumptions were made on the model. Therefore the weight function given by Equation (6.2.13) is also valid for anisotropic media:

$$
W_{3 \mathrm{D}}(\boldsymbol{\xi}, M)=\mathcal{L} \sqrt{\left|\operatorname{det} \underline{\mathrm{H}}_{\mathrm{F}}\right|} \mathrm{e}^{i \frac{\pi}{2}\left(1-\frac{\mathrm{sgn} \underline{H}_{\mathrm{F}}}{2}\right)},
$$

where, as a reminder, $\mathcal{L}$ is the geometrical spreading and $\underline{\mathrm{H}}_{\mathrm{F}}$ the Hessian matrix of the traveltime difference $\tau_{F}=\tau_{D}-\tau_{R}$.

The geometrical spreading is proportional to the square-root of the determinant of the matrix $\underline{Q}_{2}$ (see Chapter 2 and 5). Application of the results by Schleicher et al. (2001) leads to an expression for the spreading in anisotropic media which differs from the isotropic result only by a factor that acknowledges the angle between phase and group velocity:

$$
\mathcal{L}_{\text {aniso }}=\sqrt{\frac{V_{s}}{v_{s}} \frac{V_{g}}{v_{g}}} \mathcal{L}_{\text {iso }}=\sqrt{\frac{\cos \vartheta_{s} \cos \vartheta_{g}}{|\operatorname{det} \underline{N}|} \frac{V_{s}}{v_{s}} \frac{V_{g}}{v_{g}}} .
$$

The phase velocities $V_{I}$ are known directly from the slownesses, whereas the components of the group velocity vector can be determined from the slowness vectors and the elements of the density normalised elasticity tensor following Cervený and Pšenčík (1972). Figure 10.3 shows geometrical spreading for an elliptically anisotropic medium determined from traveltimes, and the corresponding errors with a median of the relative error of $0.23 \%$ and a maximum of $9.2 \%$. As for the isotropic examples, the main contributions to the errors come from the trilinear interpolation. 

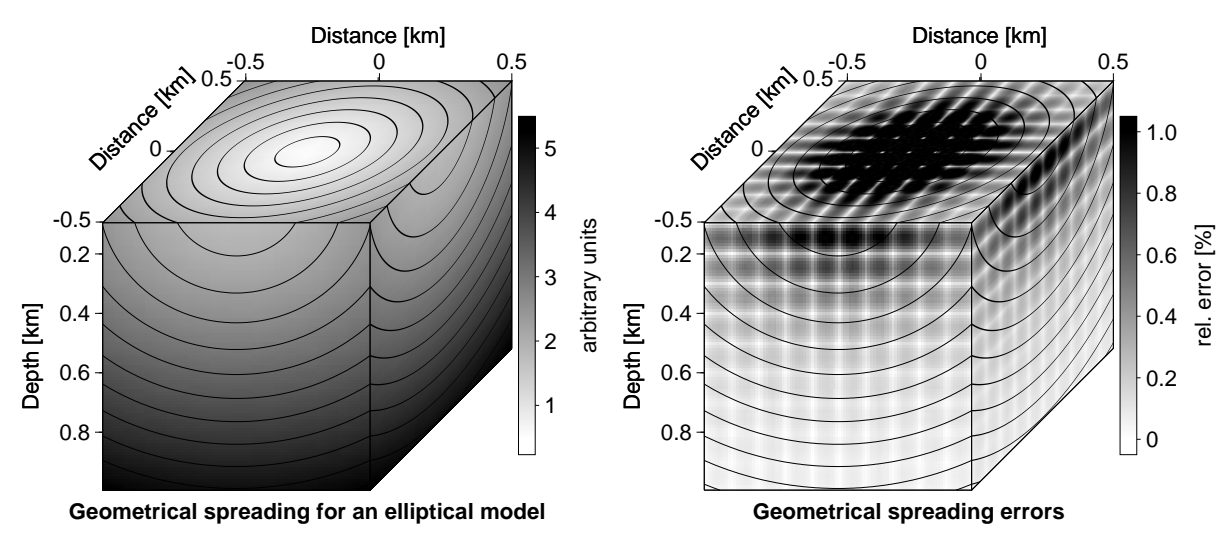

Figure 10.3: Geometrical spreading determined from traveltimes for a homogeneous velocity model with elliptical anisotropy. Left: the spreading was computed on a $100 \mathrm{~m}$ coarse grid and interpolated onto a 10m fine grid. Right: relative errors of the spreading compared to analytical results (see, e.g., Pšenčík and Teles 1996).

There exists also an expression for the decomposition of the geometrical spreading into the down- and up-going ray branches in anisotropic media, corresponding to Equation (6.4.24) (Schleicher et al., 2001). Use of these results will lead to appropriate weight functions for 3-D media with arbitrary anisotropy. 


\section{Appendix A}

\section{Nomenclature}

\section{A.1 Indices, Vectors and Matrices}

Capital indices as, e.g., $I, J, K$, take values 1 and 2 . Indices in lower case letters, as $i$, $j, k$ take values 1,2 and 3 . Unless otherwise stated, summation convention applies over repeated suffixes. A comma preceding a quantity $t$, as in $U_{, t}$, denotes differentiation with respect to $t$. If the subscript following the comma is an index $i$, as in $\tau_{, i}$, it stands for differentiation with respect to the $i^{\text {th }}$ component of the corresponding spatial vector.

A vector $\hat{\boldsymbol{a}}$ with a hat has three components, a vector $\boldsymbol{a}$ has two components. Vectors with unit length, e.g., base vectors are indicated with an arrow: $\overrightarrow{\boldsymbol{e}}$. If a superscript is given, as, e.g., in $\boldsymbol{p}^{(q)}$, the vector is to be taken in the appropriate coordinate system ( $q$ stands for ray centred coordinates).

As for vectors, matrices $\underline{\hat{A}}$ carrying a hat are $3 \times 3$ matrices. Matrices $\underline{A}$ without hat are in most cases $2 \times 2$ matrices. There are, however, exceptions, like $3 \times 2$ or $4 \times 1$ matrices. A matrix $\underline{\underline{A}}$ that is underlined twice is a $4 \times 4$ matrix, as, for example, a propagator matrix.

The $2 \times 2$ matrices $\underline{\bar{A}}$ and two component vectors $\overline{\boldsymbol{a}}$ denoted with a bar ${ }^{-}$are given in the specific coordinate system associated with the reflector surface.

A dot (".") denotes the scalar product.

\section{A.2 List of Symbols}

Some of the letters of the alphabet are used on more than one occasion. This was necessary because I wished to use the same notation as in most of the literature. It should, however, not be a problem to distinguish between the possibilities. In some cases, where a variable could not be described shortly, I have only given the reference to its introduction in the text. 


\section{A.2.1 Latin Letters}

\begin{tabular}{|c|c|}
\hline$a_{i j k l}$ & density normalised elasticity tensor \\
\hline$A$ & migration aperture \\
\hline $\mathcal{A}$ & transmission losses \\
\hline$A_{I J}, \underline{\mathrm{A}}$ & submatrix of propagator $\underline{\underline{T}}$ \\
\hline$\vec{b}$ & binormal vector \\
\hline$B_{I J}, \underline{\mathrm{B}}$ & submatrix of propagator $\underline{\underline{T}}$ \\
\hline$c_{i j k l}$ & elasticity tensor \\
\hline$C_{I J}, \underline{\mathrm{C}}$ & submatrix of propagator $\underline{T}$ \\
\hline$D_{I J}, \underline{\mathrm{D}}$ & curvature matrix of an interface \\
\hline$D_{I J}, \underline{\mathrm{D}}$ & submatrix of propagator $\underline{\underline{T}}$ \\
\hline$\vec{e}_{I}$ & base vector of the ray centred coordinate system \\
\hline$E_{I J}, \underline{E}$ & matrix describing inhomogeneities near an interface \\
\hline$F(t)$ & analytical source pulse \\
\hline$F_{I J}, \underline{\mathrm{F}}$ & see Equation (2.8.7) \\
\hline$\underline{F}$ & interface matrix \\
\hline $\bar{g}_{i}, \hat{\boldsymbol{g}}$ & receiver position vector \\
\hline 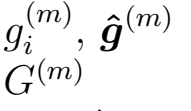 & $\begin{array}{l}m^{\text {th }} \text { eigenvector of the Christoffel matrix, polarisation vector } \\
m^{\text {th }} \text { eigenvalue of the Christoffel matrix }\end{array}$ \\
\hline$G_{i j}, \underline{\hat{\mathrm{G}}}$ & Jacobian from ray centred to interface Cartesian coordinates \\
\hline $\begin{array}{l}G_{i j}, \underline{\hat{G}} \\
h\end{array}$ & $\begin{array}{l}3 \times 32^{\text {nd }} \text { order derivative matrix of traveltimes } \\
\text { scale parameter }\end{array}$ \\
\hline $\boldsymbol{h}, h$ & half-offset coordinate \\
\hline$H_{i j}, \underline{\hat{\mathrm{H}}}$ & Jacobian from Cartesian to ray centred coordinates \\
\hline$\underline{\mathrm{H}}_{\mathrm{F}}$ & see Equation $(6.2 .8)$ \\
\hline$\underline{H}$ & Fresnel matrix, see Equation (6.3.19) \\
\hline $\mathcal{H}$ & Hamiltonian \\
\hline$i$ & imaginary unit, $i^{2}=-1$ \\
\hline $\overrightarrow{\boldsymbol{\imath}}_{i}$ & base vector of the interface Cartesian coordinate system \\
\hline$\underline{\underline{J}}$ & see Equation (2.6.13) \\
\hline$\overline{\mathcal{J}}$ & determinant of the Jacobian from Cartesian to ray coordinates \\
\hline$K$ & curvature of a ray \\
\hline$K_{\mathrm{NIP}}$ & wavefront curvature of a NIP wave \\
\hline$K_{\mathrm{N}}$ & wavefront curvature of an $\mathrm{N}$ wave \\
\hline$K_{\mathrm{CS}}^{\mathrm{G}}$ & wavefront curvature \\
\hline$K_{\mathrm{CMP}}^{\mathrm{G}}$ & wavefront curvature \\
\hline$K_{\mathrm{CMP}}^{\mathrm{S}}$ & wavefront curvature \\
\hline$L_{i}, \hat{\boldsymbol{L}}$ & vector operator \\
\hline$L$ & characteristic length, scale length \\
\hline$L$ & relative geometrical spreading \\
\hline $\mathcal{L}$ & normalised geometrical spreading \\
\hline$M_{i}, \hat{M}$ & vector operator \\
\hline$M_{I J}, \underline{\mathrm{M}}$ & see Equation (2.5.27) \\
\hline
\end{tabular}

Section 2.4

Section 6.2

Section 2.8

Section 2.9

Section 2.5

Section 2.9

Section 2.1

Section 2.9

Section 2.8

Section 2.9

Section 2.5

Section 2.8

Section 6.2

Section 2.8

Section 2.8

Section 3.1

Section 2.2

Section 2.2

Section 2.8

Section 3.1

Section 2.5

Section 8.2

Section 2.5

Section 6.2

Section 6.3

Section 2.4

Section 2.1

Section 2.8

Section 2.6

Section 2.3

Section 2.5

Section 8.3

Section 8.3

Section 8.3

Section 8.3

Section 8.3

Section 2.1

Section 2.1

Section 2.3

Section 5.2

Section 2.1

Section 2.5 


\begin{tabular}{|c|c|c|}
\hline$\vec{n}$ & normal vector & Section 2.5 \\
\hline$N_{i}, \hat{\mathbf{N}}$ & vector operator & Section 2.1 \\
\hline$N_{i j}, \underline{\hat{\mathrm{N}}}$ & $3 \times 32^{\text {nd }}$ order derivative matrix of traveltimes & Section 3.1 \\
\hline$p_{i}, \hat{\boldsymbol{p}}$ & slowness vector & Section 2.2 \\
\hline$p_{i_{0}}, \hat{\boldsymbol{p}}_{0}$ & source slowness vector (central ray) & Section 3.1 \\
\hline$\underline{P}_{1}$ & submatrix of propagator $\underline{\underline{\Pi}}$ & Section 2.6 \\
\hline$\underline{\mathrm{P}}_{2}$ & submatrix of propagator $\underline{\underline{\Pi}}$ & Section 2.6 \\
\hline$P_{I J}, \underline{\mathrm{P}}$ & see Equation (2.5.16) & Section 2.5 \\
\hline$q_{I}, \boldsymbol{q}$ & ray centred coordinates & Section 2.5 \\
\hline$q_{i_{0}}, \hat{\boldsymbol{q}}_{0}$ & receiver slowness vector (central ray) & Section 3.1 \\
\hline & submatrix of propagator $\underline{\underline{\Pi}}$ & Section 2.6 \\
\hline$\overline{\mathrm{Q}}_{2}$ & submatrix of propagator $\underline{\underline{\bar{\Pi}}}$ & Section 2.6 \\
\hline$Q_{I J}, \underline{\mathrm{Q}}$ & Upper left submatrix of $\underline{\hat{Q}}$ & Section 2.5 \\
\hline$\hat{\mathrm{Q}}_{2}^{-}$ & see Equation (2.7.13) & Section 2.7 \\
\hline$Q_{i j}, \underline{\hat{Q}}$ & Jacobian from ray centred to ray coordinates & Section 2.5 \\
\hline$\hat{\boldsymbol{r}}$ & radius vector & Section 2.5 \\
\hline$r$ & offset coordinate & Section 8.2 \\
\hline $\mathcal{R}$ & plane wave reflection coefficient & Section 6.2 \\
\hline$R_{i j}, \underline{\hat{\mathrm{R}}}$ & reflection/transmission coefficient matrix & Section 2.8 \\
\hline$s$ & arc length & Section 2.3 \\
\hline$s_{i}, \hat{\boldsymbol{s}}$ & source position vector & Section 3.1 \\
\hline$S, S_{I}, \hat{\boldsymbol{S}}$ & surface, surface vector & Section 2.3 \\
\hline$S_{i j}, \underline{\hat{S}}$ & $3 \times 32^{\text {nd }}$ order derivative matrix of traveltimes & Section 3.1 \\
\hline$\underline{\underline{S}}$ & see Equation $(2.6 .3)$ & Section 2.6 \\
\hline $\bar{t}$ & time & Section 2.1 \\
\hline$\vec{t}$ & tangent vector & Section 2.5 \\
\hline$T$ & torsion of a ray & Section 2.5 \\
\hline$\underline{\underline{\underline{\underline{T}}}}$ & $4 \times 4$ Bortfeld propagator matrix & Section 2.9 \\
\hline$\underline{\hat{\mathbf{T}}}$ & $6 \times 6$ pseudo propagator matrix & Section 4.1 \\
\hline $\bar{u}$ & see Equation (2.8.14) & Section 2.8 \\
\hline$u_{i}, \hat{\boldsymbol{u}}$ & displacement vector & Section 2.1 \\
\hline$U_{i}^{(n)}, \hat{\boldsymbol{U}}^{(n)}$ & $n^{\text {th }}$ term of vectorial displacement amplitude & Section 2.1 \\
\hline$U_{i}, \hat{\boldsymbol{U}}$ & zero order term of vectorial displacement amplitude & Section 2.1 \\
\hline$U_{T A}$ & true amplitude trace & Section 6.2 \\
\hline$v_{i}, \hat{\boldsymbol{v}}$ & group velocity vector & Section 2.3 \\
\hline$V$ & phase velocity & Section 2.2 \\
\hline$V_{\mathrm{P}}$ & $\mathrm{P}$ wave velocity & Section 2.2 \\
\hline$V_{\mathrm{S}}$ & $\mathrm{S}$ wave velocity & Section 2.2 \\
\hline$V_{\text {rms }}$ & RMS velocity & Section 8.1 \\
\hline$V_{\text {nmo }}$ & velocity & Section 8.1 \\
\hline$V$ & volume & Section 2.3 \\
\hline$V(M)$ & migration output, result of diffraction stack & Section 6.2 \\
\hline$V_{I J}, \underline{\mathrm{V}}$ & matrix of second order velocity derivatives & Section 2.5 \\
\hline
\end{tabular}


$W_{3 \mathrm{D}} \quad 3-\mathrm{D}$ weight function

Section 6.2

W $\quad 4 \times 1$ column matrix containing $\boldsymbol{q}$ and $\boldsymbol{p}^{(q)}$

Section 2.6

$x_{i}, \hat{\boldsymbol{x}} \quad$ Cartesian coordinate vector

Section 2.1

$\boldsymbol{x}_{m}, x_{m} \quad$ midpoint coordinate

Section 8.2

$\underline{\mathrm{X}} \quad 4 \times 2$ matrix containing $\underline{\mathrm{Q}}$ and $\underline{\mathrm{P}}$

Section 2.6

$X_{i j}, \underline{\hat{\mathrm{X}}} \quad$ Jacobian from Cartesian to ray coordinates

Section 2.3

$y_{i}, \hat{\boldsymbol{y}} \quad$ local Cartesian coordinates

Section 2.7

$Y_{i j}, \underline{\hat{Y}} \quad$ see Equation (2.4.7)

Section 2.4

$Z_{i j}, \underline{\hat{Z}} \quad$ Jacobian from Cartesian to interface Cartesian coordinates

Section 2.8

$z_{i}, \hat{\boldsymbol{z}}$

interface Cartesian coordinates

Section 2.8

\section{A.2.2 Greek Letters}

$\alpha \quad$ angle, see Equation (5.2.18)

Section 5.2

$\gamma_{i} \quad$ ray coordinate

$\Gamma_{i j} \quad$ Christoffel matrix

ᄃ measurement configuration matrix

Section 2.3

$\bar{\delta}_{i j} \quad$ Kronecker delta

Section 2.2

$\varepsilon_{i j} \quad$ strain tensor

$\theta \quad$ angle, see Equation (2.5.3)

$\theta \quad$ incidence angle (against surface normal)

Section 6.3

$\vartheta \quad$ ray angle against the $z$ axis

Section 2.2

Section 2.1

Section 2.5

$\kappa \quad$ index of the ray, KMAH index

Section 2.8

$\lambda \quad$ wavelength

Section 5.2

$\lambda \quad$ Lamé parameter

Section 2.3

Section 2.1

$\mu \quad$ Lamé parameter

Section 2.2

$\xi, \boldsymbol{\xi}$ trace coordinates

Section 2.2

$\xi^{*}, \boldsymbol{\xi}^{*}$ stationary point in trace coordinates

Section 6.2

$\underline{\underline{n}}$ propagator matrix in ray centred coordinates

Section 6.2

$\bar{\rho} \quad$ density

$\underline{\Sigma}$ measurement configuration matrix

Section 2.6

$\sigma_{i j} \quad$ stress tensor

Section 2.1

Section 6.3

$\tau \quad$ eikonal, phase function, traveltime

Section 2.1

$\tau_{0} \quad$ traveltime of the central ray

Section 2.1

$\tau_{R} \quad$ reflection traveltime

Section 3.1

$\tau_{D} \quad$ diffraction traveltime

Section 6.2

$\tau_{F} \quad$ difference between diffraction and reflection traveltime

Section 6.2

$\tau_{L} \quad$ signal length

Section 6.2

$\phi \quad$ angle, see Equation (2.8.3)

Section 7.1

Section 2.8

$\varphi \quad$ angle

$\varphi \quad$ azimuth angle

Section 5.2

$\Psi, \underline{\Psi} \quad$ see Equation (2.3.21)

Section 8.2

$\omega$ angular frequency

Section 2.3

$\Omega$

Section 2.3 


\section{Appendix B}

\section{Measurement Configuration Matrices}

In Sections 6.2 and 6.5 locations are expressed in trace coordinates $\left(\xi_{1}, \xi_{2}\right)$. The relationship between them and the vectors of the source and receiver coordinates $\boldsymbol{s}$ and $\boldsymbol{g}$ are given by

$$
\begin{aligned}
& \boldsymbol{s}=\boldsymbol{s}_{0}+\underline{\Sigma}\left(\boldsymbol{\xi}-\boldsymbol{\xi}^{*}\right) \\
& \boldsymbol{g}=\boldsymbol{g}_{0}+\underline{\Gamma}\left(\boldsymbol{\xi}-\boldsymbol{\xi}^{*}\right)
\end{aligned}
$$

where

$$
\Sigma_{i j}=\frac{\partial s_{i}}{\partial \xi_{j}} \quad, \quad \Gamma_{i j}=\frac{\partial g_{i}}{\partial \xi_{j}}
$$

The coordinate $\boldsymbol{\xi}^{*}$ is that of the stationary point and the constants $\boldsymbol{s}_{0}, \boldsymbol{g}_{0}$ are given by

$$
\boldsymbol{s}_{0}=\boldsymbol{s}\left(\boldsymbol{\xi}^{*}\right) \quad \text { and } \quad \boldsymbol{g}_{0}=\boldsymbol{g}\left(\boldsymbol{\xi}^{*}\right) \quad
$$

Examples for frequently used configurations are (Schleicher et al., 1993a)

- for a common shot configuration: $\underline{\Sigma}=\underline{0}$ and $\underline{\Gamma}=\underline{1}$,

- for a common receiver configuration: $\underline{\Sigma}=\underline{1}$ and $\underline{\Gamma}=\underline{0}$,

- for a common offset configuration: $\underline{\Sigma}=\underline{1}$ and $\underline{\Gamma}=\underline{1}$,

- for a common midpoint configuration: $\underline{\Sigma}=\underline{1}$ and $\underline{\Sigma}=-\underline{1}$.

More examples can be found in Vermeer (1995).

For the special case of $2.5-\mathrm{D}, \xi_{1}$ is taken to be the in-plane and $\xi_{2}$ the out-of-plane direction with the sources and receivers being both positioned at $\xi_{2}^{*}$. This corresponds to a zerooffset configuration in $\xi_{2}$-direction and therefore in this case the configuration matrices $\underline{\Sigma}$ and $\underline{I}$ reduce to

$$
\underline{\Sigma}=\left(\begin{array}{cc}
\Sigma_{11} & 0 \\
0 & 1
\end{array}\right) \quad, \quad \underline{\Gamma}=\left(\begin{array}{cc}
\Gamma_{11} & 0 \\
0 & 1
\end{array}\right),
$$

where only the first elements are configuration-dependent. For example, in a common shot experiment

$$
\Sigma_{11}=0 \quad, \quad \Gamma_{11}=1 .
$$




\section{Appendix C}

\section{Analytic Coefficients for Simple Models}

This appendix contains expressions for the traveltimes and their first and second-order derivatives for simple cases where the analytic solution is known. For the generic examples in isotropic media I have chosen a model with a constant vertical velocity gradient and a homogeneous (constant velocity) medium, which is a special case of the constant velocity gradient medium. Expressions for the traveltime and the coefficients are also given for a homogeneous medium with elliptical anisotropy. For both homogeneous models given here, the hyperbolic traveltime expression yields the exact traveltime. This is explicitly shown for the isotropic homogeneous model.

For all three models I denote the source position by $\left(s_{x}, s_{y}, s_{z}\right)$ and the receiver position by $\left(g_{x}, g_{y}, g_{z}\right)$. I use the following abbreviations: $x=g_{x}-s_{x}$, etc., and $r=\sqrt{x^{2}+y^{2}+z^{2}}$.

\section{C.1 Constant Velocity Gradient Model}

This velocity model is described by the vertical gradient $b$ and

$$
V(z)=a+b z
$$

Form this follow the velocity at the source $V_{s}=a+b s_{z}$ and at the receiver $V_{g}=a+b g_{z}$. The traveltime from $\hat{\boldsymbol{s}}$ to $\hat{\boldsymbol{g}}$ is

$$
\tau=\frac{1}{b} \operatorname{arcosh}\left(1+\frac{b^{2} r^{2}}{2 V_{s} V_{g}}\right)
$$

The geometrical spreading in two and three dimensions is

$$
\begin{aligned}
& \mathcal{L}_{2 \mathrm{D}}=\frac{1}{V_{s}} \sqrt{\frac{W}{2}}, \quad \text { and } \\
& \mathcal{L}_{3 \mathrm{D}}=\frac{1}{V_{s}} \frac{W}{2},
\end{aligned}
$$


where the abbreviation

$$
W=\sqrt{b^{2} r^{4}+4 V_{s} V_{g} r^{2}}
$$

was used. To simplify the expressions for the coefficients, another abbreviation is introduced here:

$$
F=b^{2} r^{2}+2 V_{s} V_{g}
$$

The first order derivatives of Equation (C.1.2) lead to the slowness components are

$$
\begin{aligned}
p_{x_{0}}=q_{x_{0}} & =\frac{1}{W} 2 x \\
p_{y_{0}}=q_{y_{0}} & =\frac{1}{W} 2 y \\
p_{z_{0}} & =\frac{1}{W}\left(2 z+\frac{b r^{2}}{V_{s}}\right) \\
q_{z_{0}} & =\frac{1}{W}\left(2 z-\frac{b r^{2}}{V_{g}}\right)
\end{aligned}
$$

The second order derivative matrix elements are

$$
\begin{aligned}
S_{x x}=-G_{x x}=-N_{x x} & =\frac{1}{W^{3}}\left(4 F x^{2}-2 W^{2}\right), \\
S_{y y}=-G_{y y}=-N_{y y} & =\frac{1}{W^{3}}\left(4 F y^{2}-2 W^{2}\right), \\
S_{x y}=-G_{x y}=-N_{x y}=-N_{y x} & =\frac{1}{W^{3}}(4 F x y), \\
S_{y z}=-N_{z y} & =\frac{1}{W^{3}}\left(4 F y z-4 V_{g} b r^{2} y\right), \\
G_{y z}=N_{y z} & =\frac{1}{W^{3}}\left(-4 F y z-4 V_{s} b r^{2} y\right) \\
S_{z x}=-N_{z x} & =\frac{1}{W^{3}}\left(4 F z x-4 V_{g} b r^{2} x\right), \\
G_{z x}=N_{x z} & =\frac{1}{W^{3}}\left(-4 F z x-4 V_{s} b r^{2} x\right)
\end{aligned}
$$

and

$$
\begin{aligned}
S_{z z} & =\frac{1}{W^{3}}\left(4 F z^{2}-2 W^{2}-\frac{b^{4} r^{6}}{V_{s}^{2}}-\frac{6 V_{g} b^{2} r^{4}}{V_{s}}+8 V_{g}\left(V_{s}-V_{g}\right) r^{2}\right) \\
G_{z z} & =\frac{1}{W^{3}}\left(-4 F z^{2}+2 W^{2}+\frac{b^{4} r^{6}}{V_{g}^{2}}+\frac{6 V_{s} b^{2} r^{4}}{V_{g}}-8 V_{s}\left(V_{g}-V_{s}\right) r^{2}\right) \\
N_{z z} & =\frac{8 V_{g} V_{s}}{W^{3}}\left(r^{2}-z^{2}\right)
\end{aligned}
$$

\section{C.2 Homogeneous Isotropic Model}

This model is a special case of the constant velocity gradient model with $b=0$. The constant velocity is denoted by $V$. Application of the formula of l'Hospital to Equation 
(C.1.2) leads to the well-known result

$$
\tau=\frac{r}{V}
$$

With $W=2 V r$, the geometrical spreading for this homogeneous model is given by

$$
\begin{aligned}
\mathcal{L}_{2 \mathrm{D}} & =\sqrt{\frac{r}{V}}, \quad \text { and } \\
\mathcal{L}_{3 \mathrm{D}} & =\frac{r}{V},
\end{aligned}
$$

in two and three dimensions, respectively. Furthermore, with $F=2 V^{2}$, Equation (C.1.6) leads to

$$
\begin{aligned}
& p_{x_{0}}=q_{x_{0}}=\frac{x}{V r}, \\
& p_{y_{0}}=q_{y_{0}}=\frac{y}{V r}, \\
& p_{z_{0}}=q_{z_{0}}=\frac{z}{V r} .
\end{aligned}
$$

Equations (C.1.7) and (C.1.7) yield

$$
\begin{array}{r}
-S_{x x}=G_{x x}=N_{x x}=\frac{y^{2}+z^{2}}{V r^{3}}, \\
-S_{y y}=G_{y y}=N_{y y}=\frac{z^{2}+x^{2}}{V r^{3}}, \\
-S_{z z}=G_{z z}=N_{z z}=\frac{x^{2}+y^{2}}{V r^{3}}, \\
-S_{x y}=G_{x y}=N_{x y}=N_{y x}=-\frac{x y}{V r^{3}}, \\
-S_{y z}=G_{y z}=N_{y z}=N_{z y}=-\frac{y z}{V r^{3}}, \\
-S_{z x}=G_{z x}=N_{z x}=N_{x z}=-\frac{z x}{V r^{3}},
\end{array}
$$

I will now prove that the hyperbolic traveltime expansion Equation (3.2.2) is the exact solution for a model with constant velocity. Instead of vector notation I will use index notation here (summation convention is applied) with the index 1 for $x, 2$ for $y$, and 3 for $z$. Abbreviating $r_{i}=g_{i}-s_{i}$ leads to $r^{2}=r_{i} r_{i}$ and $V^{2} \tau^{2}(\hat{\boldsymbol{s}}, \hat{\boldsymbol{g}})=r_{i} r_{i}$. Equation (3.2.2) in index notation reads

$$
\tau^{2}(\hat{\boldsymbol{s}}, \hat{\boldsymbol{g}})=\left(\tau_{0}+q_{0 i} \Delta g_{i}-p_{0 i} \Delta s_{i}\right)^{2}-2 \tau_{0} \Delta s_{i} N_{i j} \Delta g_{j}+\tau_{0}\left(\Delta g_{i} G_{i j} \Delta g_{j}-\Delta s_{i} S_{i j} \Delta s_{j}\right)
$$

where the traveltime $\tau_{0}$ is taken in the expansion point and $s_{i_{0}}, g_{i_{0}}$

$$
\tau_{0}^{2}=\frac{r_{i_{0}} r_{i_{0}}}{V^{2}}=\frac{\left(g_{i_{0}}-s_{i_{0}}\right)\left(g_{i_{0}}-s_{i_{0}}\right)}{V^{2}}
$$


In this context the coefficients of Equation (C.2.12) are written as

$$
\begin{aligned}
p_{i_{0}}=q_{i_{0}} & =\frac{r_{i_{0}}}{V^{2} \tau_{0}} \\
S_{i j} & =\frac{p_{i_{0}} p_{j_{0}}}{\tau_{0}}-\frac{\delta_{i j}}{V^{2} \tau_{0}} \\
G_{i j} & =\frac{\delta_{i j}}{V^{2} \tau_{0}}-\frac{q_{i_{0}} q_{j_{0}}}{\tau_{0}} \\
N_{i j} & =\frac{\delta_{i j}}{V^{2} \tau_{0}}-\frac{p_{i_{0}} q_{j 0}}{\tau_{0}} .
\end{aligned}
$$

Inserting these into Equation (C.2.12) and expanding the square on the right side of the equation yields

$$
\begin{aligned}
\tau^{2}(\hat{\boldsymbol{s}}, \hat{\boldsymbol{g}})= & \tau_{0}^{2}+2 \tau_{0}\left(q_{i_{0}} \Delta g_{i}-p_{i_{0}} \Delta s_{i}\right)+q_{i_{0}} \Delta g_{i} q_{j_{0}} \Delta g_{j}+p_{i_{0}} \Delta s_{i} p_{j_{0}} \Delta s_{j} \\
& -2 p_{i_{0} \Delta s_{i}} q_{j_{0}} \Delta g_{j}-q_{i_{0}} \Delta g_{i} q_{j_{0}} \Delta g_{j}-p_{i_{0}} \Delta s_{i} p_{j_{0}} \Delta s_{j}+2 p_{i_{0}} \Delta s_{i} q_{j_{0}} \Delta g_{j} \\
& +\frac{\Delta g_{i} \Delta g_{i}}{V^{2}}+\frac{\Delta s_{i} \Delta s_{i}}{V^{2}}-2 \frac{\Delta s_{i} \Delta g_{i}}{V^{2}} \\
= & \tau_{0}^{2}+2 \tau_{0} q_{i_{0}} \Delta g_{i}-2 \tau_{0} p_{i_{0}} \Delta s_{i}+\frac{1}{V^{2}}\left(\Delta g_{i} \Delta g_{i}+\Delta s_{i} \Delta s_{i}-2 \Delta s_{i} \Delta g_{i}\right) \\
= & \frac{1}{V^{2}}\left(r_{i_{0}} r_{i_{0}}+2 r_{i_{0}} \Delta g_{i}-2 r_{i_{0} \Delta s_{i}}+\Delta g_{i} \Delta g_{i}+\Delta s_{i} \Delta s_{i}-2 \Delta s_{i} \Delta g_{i}\right) \\
= & \frac{1}{V^{2}}\left(r_{i_{0}}+\Delta g_{i}-\Delta s_{i}\right)\left(r_{i_{0}}+\Delta g_{i}-\Delta s_{i}\right) .
\end{aligned}
$$

This last result is the square of equation (C.2.12) and thus the hyperbolic approximation (3.2.2) yields the exact result for an isotropic medium with constant velocity.

\section{C.3 Homogeneous Model with Elliptical Anisotropy}

Elliptical anisotropy is a special case of transversal isotropic (TI) media. A TI medium is characterised by the density $\rho$ and six non-zero elastic parameters, where five of them are independent:

$$
\begin{aligned}
& c_{1111}=c_{2222}, \\
& c_{3333}, \\
& c_{1122} \quad, \\
& c_{3311}=c_{2233}, \\
& c_{2323}=c_{3131}, \text { and } \\
& c_{1212}=\frac{c_{1111}-c_{1122}}{2} .
\end{aligned}
$$

For elliptical anisotropy it is furthermore necessary that (Helbig, 1983)

$$
\left(c_{1111}-c_{2323}\right)\left(c_{3333}-c_{2323}\right)-\left(c_{3311}+c_{2323}\right)^{2}=0 \quad .
$$


Following Daley and Hron (1979a,b), the traveltime in a homogeneous elliptical anisotropic medium is

$$
\tau=\sqrt{\frac{x^{2}}{v_{x}^{2}}+\frac{y^{2}}{v_{x}^{2}}+\frac{z^{2}}{v_{z}^{2}}} .
$$

The velocity $v_{z}$ is that of a wave which travels in $z$ direction, and $v_{x}$ is that of a wave travelling in $x$ direction, or, more precisely, in the $x-y$ plane, since the medium shows rotational symmetry with respect to the $z$ axis. Please note that in this type of medium ray (group) and phase velocity coincide for waves that travel in $z$ direction or in the $x-y$ plane. The velocities $v_{x}$ and $v_{z}$ depend on the wave-type. For a $\mathrm{qP}$ wave they are given by

$$
v_{x}=\sqrt{\frac{c_{1111}}{\rho}} \text { and } v_{z}=\sqrt{\frac{c_{3333}}{\rho}} .
$$

For a qSH wave the velocities are

$$
v_{x}=\sqrt{\frac{c_{1212}}{\rho}} \text { and } v_{z}=\sqrt{\frac{c_{2323}}{\rho}} .
$$

In the case of a qSV wave both velocities are equal:

$$
v_{x}=v_{z}=\sqrt{\frac{c_{2323}}{\rho}} .
$$

The slownesses for this model are

$$
\begin{aligned}
& p_{x_{0}}=q_{x_{0}}=\frac{x}{v_{x}^{2} \tau}, \\
& p_{y_{0}}=q_{y_{0}}=\frac{y}{v_{x}^{2} \tau}, \\
& p_{z_{0}}=q_{z_{0}}=\frac{z}{v_{z}^{2} \tau} .
\end{aligned}
$$

The second-order derivatives are

$$
\begin{array}{r}
-S_{x x}=G_{x x}=N_{x x}=\frac{v_{x}^{2} \tau^{2}-x^{2}}{v_{x}^{4} \tau^{3}}, \\
-S_{y y}=G_{y y}=N_{y y}=\frac{v_{x}^{2} \tau^{2}-y^{2}}{v_{x}^{4} \tau^{3}}, \\
-S_{z z}=G_{z z}=N_{z z}=\frac{v_{z}^{2} \tau^{2}-z^{2}}{v_{z}^{4} \tau^{3}}, \\
-S_{x y}=G_{x y}=N_{x y}=N_{y x}=-\frac{x y}{v_{x}^{4} \tau^{3}}, \\
-S_{y z}=G_{y z}=N_{y z}=N_{z y}=-\frac{y z}{v_{x}^{2} v_{z}^{2} \tau^{3}}, \\
-S_{z x}=G_{z x}=N_{z x}=N_{x z}=-\frac{z x}{v_{z}^{2} v_{x}^{2} \tau^{3}},
\end{array}
$$

Similarly as for isotropic homogeneous media, it can be shown that the hyperbolic traveltime expansion yields the exact solution for a homogeneous medium with elliptical anisotropy. 


\section{Appendix D}

\section{Expressions for the Determination of the Coefficients}

This appendix gives the explicit formulae for the determination of the parabolic and hyperbolic coefficients of the $3-\mathrm{D}$ traveltime expressions. Since this requires traveltimes for different source-receiver combinations, the following notation is introduced to abbreviate the traveltimes at the various grid points of the source and receiver: $\tau_{i j k}^{I J K}$ is the traveltime from a source at the grid point denoted by $I$ (in $x$ ), $J$ (in $y$ ), and $K$ (in $z$ ) to a receiver at the grid point indicated by $i$ (in $x$ ), $j$ (in $y$ ), and $j$ (in $z$ ). The source is always assumed to be at the top of the model. Otherwise the sign of the slowness component $p_{z}$ must be taken into consideration. The indices take the values

- $m$ (for minus) for one grid point less than the one of the central ray,

- o for the grid point that corresponds to the central ray,

- $p$ (for $p l u s$ ) for one grid point more than the one of the central ray.

(See Figure D.1.) The distance between two source points is $s_{x}$ in $x$-direction and $s_{y}$ (in $y$ ). Similarly, the distance between two receiver grid points is given by $g_{x}$ (in $x$ ), $g_{y}$ (in $y$ ), and $g_{z}$ (in $z$ ). The traveltimes for the source at the central position (ooo) are abbreviated as

$$
\tau_{i j k}^{0}=\tau_{i j k}^{o o o}
$$

those with the receiver at the central position as

$$
\tau_{0}^{I J K}=\tau_{\text {ooo }}^{I J K}
$$

and that of the central ray by

$$
\tau_{0}=\tau_{\text {ooo }}^{o o o}
$$

A similar notation is used for the grid points of the velocity field: $v_{i j k}$ is the velocity at the grid point given by the indices $i, j$, and $k$, which take the same values $m, o, p$ as the indices for the traveltimes. The velocity derivatives are taken at the source position. 


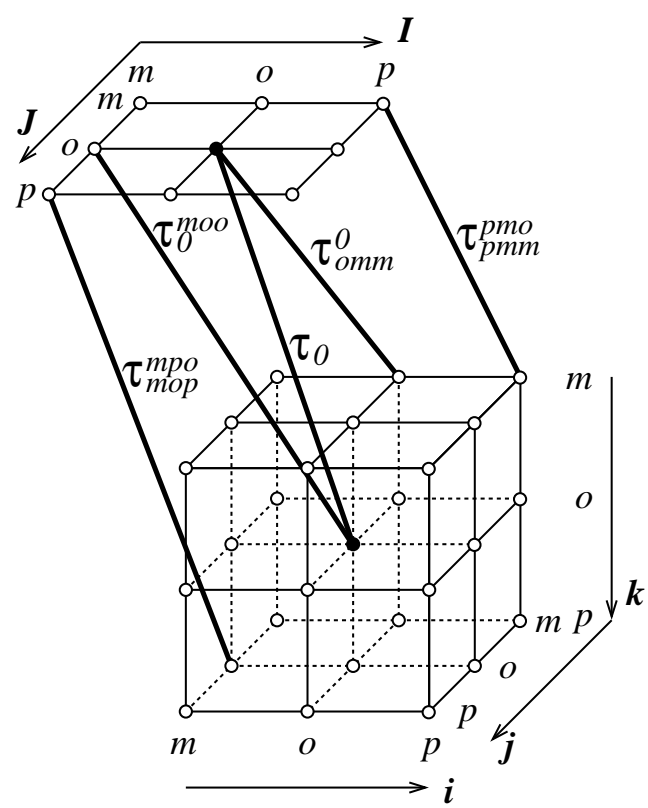

Figure D.1: Nomenclature for the traveltimes between grid points.

They are denoted and computed by

$$
\begin{aligned}
& v_{x}=\left.\frac{\partial v}{\partial x}\right|_{\hat{\boldsymbol{s}}_{0}}=\frac{v_{\text {poo }}-v_{\text {moo }}}{2 d_{x}} \\
& v_{y}=\left.\frac{\partial v}{\partial y}\right|_{\hat{\boldsymbol{s}}_{0}}=\frac{v_{\text {opo }}-v_{\text {omo }}}{2 d_{y}} \\
& v_{z}=\left.\frac{\partial v}{\partial z}\right|_{\hat{\boldsymbol{s}}_{0}}=\frac{v_{\text {oop }}-v_{0}}{d_{z}},
\end{aligned}
$$

where $d_{x}, d_{y}$, and $d_{z}$ are the grid spacings of the velocity field. The velocity at the source is abbreviated by $v_{0}$.

\section{D.1 Parabolic Coefficients for Isotropic Media}

Slowness components at the receiver, $\hat{\boldsymbol{q}}_{0}$ :

$$
\begin{aligned}
& q_{x}=\frac{\tau_{\text {poo }}^{0}-\tau_{\text {moo }}^{0}}{2 g_{x}}, \\
& q_{y}=\frac{\tau_{\text {opo }}^{0}-\tau_{\text {omo }}^{0}}{2 g_{y}}, \\
& q_{z}=\frac{\tau_{\text {oop }}^{0}-\tau_{\text {oom }}^{0}}{2 g_{z}} .
\end{aligned}
$$


Slowness components at the source, $\hat{\boldsymbol{p}}_{0}$ :

$$
\begin{aligned}
& p_{x}=\frac{\tau_{0}^{m o o}-\tau_{0}^{\text {poo }}}{2 s_{x}}, \\
& p_{y}=\frac{\tau_{0}^{o m o}-\tau_{0}^{o p o}}{2 s_{y}}, \\
& p_{z}=\sqrt{\frac{1}{v_{0}^{2}}-p_{x}^{2}-p_{y}^{2}} .
\end{aligned}
$$

Second-order derivative matrix at the receiver, $\underline{\mathrm{G}}$ :

$$
\begin{aligned}
& G_{x x}=\frac{\tau_{p o o}^{0}+\tau_{m o o}^{0}-2 \tau_{0}}{g_{x}^{2}}, \\
& G_{y y}=\frac{\tau_{o p o}^{0}+\tau_{o m o}^{0}-2 \tau_{0}}{g_{y}^{2}}, \\
& G_{z z}=\frac{\tau_{o o p}^{0}+\tau_{o o m}^{0}-2 \tau_{0}}{g_{z}^{2}}, \\
& G_{x y}=\frac{\tau_{p p o}^{0}+\tau_{m m o}^{0}-\tau_{p m o}^{0}-\tau_{m p o}^{0}}{4 g_{x} g_{y}}, \\
& G_{y z}=\frac{\tau_{o p p}^{0}+\tau_{o m m}^{0}-\tau_{o p m}^{0}-\tau_{o m p}^{0}}{4 g_{y} g_{z}}, \\
& G_{z x}=\frac{\tau_{p o p}^{0}+\tau_{m o m}^{0}-\tau_{p o m}^{0}-\tau_{m o p}^{0}}{4 g_{z} g_{x}},
\end{aligned}
$$

Second-order derivative matrix at the source, $\underline{\mathrm{S}}$ :

$$
\begin{aligned}
S_{x x} & =-\frac{\tau_{0}^{p o o}+\tau_{0}^{m o o}-2 \tau_{0}}{s_{x}^{2}}, \\
S_{y y} & =-\frac{\tau_{0}^{o p o}+\tau_{0}^{o m o}-2 \tau_{0}}{s_{y}^{2}}, \\
S_{z z} & =-\frac{d v z}{v_{0}^{3} p_{z}}-\frac{p_{x}}{p_{z}} S_{z x}-\frac{p_{y}}{p_{z}} S_{y z}, \\
S_{x y} & =-\frac{\tau_{0}^{p p o}+\tau_{0}^{m m o}-\tau_{0}^{p m o}-\tau_{0}^{m p o}}{4 s_{x} S_{y}}, \\
S_{y z} & =-\frac{v_{y}}{v_{0}^{3} p_{z}}-\frac{p_{x}}{p_{z}} S_{x y}-\frac{p_{y}}{p_{z}} S_{y y}, \\
S_{z x} & =-\frac{v_{x}}{v_{0}^{3} p_{z}}-\frac{p_{x}}{p_{z}} S_{x x}-\frac{p_{y}}{p_{z}} S_{x y},
\end{aligned}
$$


Second-order mixed derivative matrix, $\underline{\mathrm{N}}$ :

$$
\begin{aligned}
& N_{x x}=\frac{\tau_{\text {moo }}^{\text {poo }}+\tau_{\text {poo }}^{\text {moo }}-\tau_{\text {poo }}^{\text {poo }}-\tau_{\text {moo }}^{\text {moo }}}{4 s_{x} g_{x}}, \\
& N_{y y}=\frac{\tau_{\text {omo }}^{\text {opo }}+\tau_{\text {opo }}^{\text {omo }}-\tau_{\text {opo }}^{\text {opo }}-\tau_{\text {omo }}^{\text {omo }}}{4 s_{y} g_{y}}, \\
& N_{z z}=-\frac{p_{x} N_{x z}+p_{y} N_{y z}}{p_{z}}, \\
& N_{x y}=\frac{\tau_{\text {omo }}^{\text {poo }}+\tau_{\text {opo }}^{\text {moo }}-\tau_{\text {opo }}^{\text {poo }}-\tau_{\text {omo }}^{\text {moo }}}{4 s_{x} g_{y}}, \\
& N_{y x}=\frac{\tau_{\text {moo }}^{\text {opo }}+\tau_{\text {poo }}^{\text {omo }}-\tau_{\text {poo }}^{\text {opo }}-\tau_{\text {moo omo }}^{\text {omo }}}{4 s_{y} g_{x}}, \\
& N_{y z}=\frac{\tau_{\text {oom }}^{\text {opo }}+\tau_{\text {oop }}^{\text {omo }}-\tau_{\text {oop }}^{\text {opo }}-\tau_{\text {oom }}^{\text {omo }}}{4 s_{y} g_{z}}, \\
& N_{z y}=-\frac{p_{x}}{p_{z}} N_{x y}-\frac{p_{y}}{p_{z}} N_{y y}, \\
& N_{z x}=-\frac{p_{x}}{p_{z}} N_{x x}-\frac{p_{y}}{p_{z}} N_{y x}, \\
& N_{x z}=\frac{\tau_{\text {oom }}^{\text {ooo }}+\tau_{\text {oop }}^{\text {moo }}-\tau_{\text {oop }}^{\text {poo }}-\tau_{\text {oom }}^{\text {moo }}}{4 s_{x} g_{z}}
\end{aligned} .
$$

\section{D.2 Hyperbolic Coefficients for Isotropic Media}

Slowness components at the receiver, $\hat{\boldsymbol{q}}_{0}$ :

$$
\begin{aligned}
& q_{x}=\frac{\left(\tau_{\text {poo }}^{0}\right)^{2}-\left(\tau_{\text {moo }}^{0}\right)^{2}}{4 g_{x} \tau_{0}}, \\
& q_{y}=\frac{\left(\tau_{\text {opo }}^{0}\right)^{2}-\left(\tau_{\text {omo }}^{0}\right)^{2}}{4 g_{y} \tau_{0}}, \\
& q_{z}=\frac{\left(\tau_{\text {oop }}^{0}\right)^{2}-\left(\tau_{\text {oom }}^{0}\right)^{2}}{4 g_{z} \tau_{0}},
\end{aligned}
$$

Slowness components at the source, $\hat{\boldsymbol{p}}_{0}$ :

$$
\begin{aligned}
& p_{x}=\frac{\left(\tau_{0}^{\text {moo }}\right)^{2}-\left(\tau_{0}^{\text {poo }}\right)^{2}}{4 s_{x} \tau_{0}}, \\
& p_{y}=\frac{\left(\tau_{0}^{\text {omo }}\right)^{2}-\left(\tau_{0}^{\text {opo }}\right)^{2}}{4 s_{y} \tau_{0}}, \\
& p_{z}=\sqrt{\frac{1}{v_{0}^{2}}-p_{x}^{2}-p_{y}^{2}} .
\end{aligned}
$$


Second-order derivative matrix at the receiver, $\underline{G}$ :

$$
\begin{aligned}
& G_{x x}=\frac{\left(\tau_{\text {poo }}^{0}\right)^{2}+\left(\tau_{\text {moo }}^{0}\right)^{2}-2 \tau_{0}^{2}}{2 g_{x}^{2} \tau_{0}}-\frac{q_{x}^{2}}{\tau_{0}}, \\
& G_{y y}=\frac{\left(\tau_{o p o}^{0}\right)^{2}+\left(\tau_{o m o}^{0}\right)^{2}-2 \tau_{0}^{2}}{2 g_{y}^{2} \tau_{0}}-\frac{q_{y}^{2}}{\tau_{0}}, \\
& G_{z z}=\frac{\left(\tau_{o o p}^{0}\right)^{2}+\left(\tau_{\text {oom }}^{0}\right)^{2}-2 \tau_{0}^{2}}{2 g_{z}^{2} \tau_{0}}-\frac{q_{z}^{2}}{\tau_{0}}, \\
& G_{x y}=\frac{\left(\tau_{p p o}^{0}\right)^{2}+\left(\tau_{m m o}^{0}\right)^{2}-\left(\tau_{p m o}^{0}\right)^{2}-\left(\tau_{m p o}^{0}\right)^{2}}{8 g_{x} g_{y} \tau_{0}}-\frac{q_{x} q_{y}}{\tau_{0}} \\
& G_{y z}=\frac{\left(\tau_{o p p}^{0}\right)^{2}+\left(\tau_{o m m}^{0}\right)^{2}-\left(\tau_{o p m}^{0}\right)^{2}-\left(\tau_{o m p}^{0}\right)^{2}}{8 g_{y} g_{z} \tau_{0}}-\frac{q_{y} q_{z}}{\tau_{0}} \\
& G_{z x}=\frac{\left(\tau_{\text {pop }}^{0}\right)^{2}+\left(\tau_{m o m}^{0}\right)^{2}-\left(\tau_{\text {pom }}^{0}\right)^{2}-\left(\tau_{m o p}^{0}\right)^{2}}{8 g_{z} g_{x} \tau_{0}}-\frac{q_{z} q_{x}}{\tau_{0}}
\end{aligned}
$$

Second-order derivative matrix at the source, $\underline{\mathrm{S}}$ :

$$
\begin{aligned}
& S_{x x}=\frac{-\left(\tau_{0}^{\text {poo }}\right)^{2}-\left(\tau_{0}^{m o o}\right)^{2}+2 \tau_{0}^{2}}{2 s_{x}^{2} \tau_{0}}+\frac{p_{x}^{2}}{\tau_{0}} \\
& S_{y y}=\frac{-\left(\tau_{0}^{o p o}\right)^{2}-\left(\tau_{0}^{o m o}\right)^{2}+2 \tau_{0}^{2}}{2 s_{y}^{2} \tau_{0}}+\frac{p_{y}^{2}}{\tau_{0}}, \\
& S_{z z}=-\frac{v_{z}}{v_{0}^{3} p_{z}}-\frac{p_{x}}{p_{z}} S_{z x}-\frac{p_{y}}{p_{z}} S_{y z}, \\
& S_{x y}=\frac{-\left(\tau_{0}^{p p o}\right)^{2}-\left(\tau_{0}^{m m o}\right)^{2}+\left(\tau_{0}^{p m o}\right)^{2}+\left(\tau_{0}^{m p o}\right)^{2}}{8 s_{x} s_{y} \tau_{0}}+\frac{p_{x} p_{y}}{\tau_{0}} \\
& S_{y z}=-\frac{v_{y}}{v_{0}^{3} p_{z}}-\frac{p_{x}}{p_{z}} S_{x y}-\frac{p_{y}}{p_{z}} S_{y y} \\
& S_{z x}=-\frac{v_{x}}{v_{0}^{3} p_{z}}-\frac{p_{x}}{p_{z}} S_{x x}-\frac{p_{y}}{p_{z}} S_{x y}
\end{aligned}
$$


Second-order mixed derivative matrix, $\underline{\mathrm{N}}$ :

$$
\begin{aligned}
& N_{x x}=\frac{\left(\tau_{\text {moo }}^{\text {poo }}\right)^{2}+\left(\tau_{\text {poo }}^{\text {moo }}\right)^{2}-\left(\tau_{\text {poo }}^{\text {poo }}\right)^{2}-\left(\tau_{\text {moo }}^{\text {moo }}\right)^{2}}{8 s_{x} g_{x} \tau_{0}}-\frac{p_{x} q_{x}}{\tau_{0}}, \\
& N_{y y}=\frac{\left(\tau_{o m o}^{o p o}\right)^{2}+\left(\tau_{o p o}^{o m o}\right)^{2}-\left(\tau_{o p o}^{o p o}\right)^{2}-\left(\tau_{o m o}^{o m o}\right)^{2}}{8 s_{y} g_{y} \tau_{0}}-\frac{p_{y} q_{y}}{\tau_{0}}, \\
& N_{z z}=-\frac{p_{x} N_{x z}+p_{y} N_{y z}}{p_{z}} \\
& N_{x y}=\frac{\left(\tau_{\text {omo }}^{\text {poo }}\right)^{2}+\left(\tau_{\text {opo }}^{\text {moo }}\right)^{2}-\left(\tau_{\text {opo }}^{\text {poo }}\right)^{2}-\left(\tau_{\text {omo }}^{\text {moo }}\right)^{2}}{8 s_{x} g_{y} \tau_{0}}-\frac{p_{x} q_{y}}{\tau_{0}}, \\
& N_{y x}=\frac{\left(\tau_{\text {moo }}^{\text {opo }}\right)^{2}+\left(\tau_{\text {poo }}^{\text {omo }}\right)^{2}-\left(\tau_{\text {poo }}^{\text {opo }}\right)^{2}-\left(\tau_{\text {moo }}^{\text {omo }}\right)^{2}}{8 s_{y} g_{x} \tau_{0}}-\frac{p_{y} q_{x}}{\tau_{0}}, \\
& N_{y z}=\frac{\left(\tau_{\text {oom }}^{\text {opo }}\right)^{2}+\left(\tau_{\text {oop }}^{\text {omo }}\right)^{2}-\left(\tau_{\text {oop }}^{\text {opo }}\right)^{2}-\left(\tau_{\text {oom }}^{\text {omo }}\right)^{2}}{8 s_{y} g_{z} \tau_{0}}-\frac{p_{y} q_{z}}{\tau_{0}}, \\
& N_{z y}=-\frac{p_{x}}{p_{z}} N_{x y}-\frac{p_{y}}{p_{z}} N_{y y}, \\
& N_{z x}=-\frac{p_{x}}{p_{z}} N_{x x}-\frac{p_{y}}{p_{z}} N_{y x}, \\
& N_{x z}=\frac{\left(\tau_{\text {oom }}^{\text {poo }}\right)^{2}+\left(\tau_{\text {oop }}^{\text {moo }}\right)^{2}-\left(\tau_{\text {oop }}^{\text {poo }}\right)^{2}-\left(\tau_{\text {oom }}^{\text {moo }}\right)^{2}}{8 s_{x} g_{z} \tau_{0}}-\frac{p_{x} q_{z}}{\tau_{0}} .
\end{aligned}
$$

\section{D.3 Coefficients for Anisotropic Media}

In anisotropic media, the expressions given above for coefficients which contain derivatives with respect to the $z$ position of the source are no longer valid, because the isotropic eikonal equation was used for their derivation. At this time, the determination of these derivatives for anisotropic media requires that traveltimes are given for source positions that are below or above the position of the central source grid point. An approach to the derivation of formulae to express the $z$-components of the source derivatives in term of already computed matrix elements is outlined in Section 10.3. Until these formulae exist, however, the coefficients under consideration can be determined from the following expressions. 


\section{Parabolic Coefficients}

Equations (D.1.10), (D.1.19), (D.1.21), (D.1.22), (D.1.25), (D.1.29), and (D.1.30) are replaced by the following expressions:

$$
\begin{aligned}
p_{z} & =\frac{\tau_{0}^{\text {oom }}-\tau_{0}^{\text {oop }}}{2 s_{z}}, \\
S_{z z} & =-\frac{\tau_{0}^{o o p}+\tau_{0}^{o o m}-2 \tau_{0}}{s_{z}^{2}}, \\
S_{y z} & =-\frac{\tau_{0}^{o p p}+\tau_{0}^{o m m}-\tau_{0}^{o p m}-\tau_{0}^{o m p}}{4 s_{y} s_{z}} \\
S_{z x} & =-\frac{\tau_{0}^{\text {pop }}+\tau_{0}^{\text {mom }}-\tau_{0}^{\text {oom }}-\tau_{0}^{\text {mop }}}{4 s_{z} s_{x}} \\
N_{z z} & =\frac{\tau_{\text {oom }}^{\text {oop }}+\tau_{\text {oop }}^{\text {oom }}-\tau_{\text {oop }}^{\text {oop }}-\tau_{\text {oom }}^{\text {oom }}}{4 s_{z} g_{z}} \\
N_{z x} & =\frac{\tau_{m o o}^{\text {oop }}+\tau_{\text {poo }}^{\text {oom }}-\tau_{\text {poo }}^{\text {oop }}-\tau_{\text {moo }}^{\text {oom }}}{4 s_{z} g_{x}} \\
N_{z y} & =\frac{\tau_{\text {omo }}^{\text {oop }}+\tau_{\text {opo }}^{\text {oom }}-\tau_{\text {opo }}^{\text {oop }}-\tau_{\text {omo }}^{\text {oom }}}{4 s_{z} g_{y}}
\end{aligned}
$$

\section{Hyperbolic Coefficients}

Equations (D.2.37), (D.2.46), (D.2.48), (D.2.49), (D.2.52), (D.2.56), and (D.2.57) are replaced by the following expressions:

$$
\begin{aligned}
p_{z} & =\frac{\left(\tau_{0}^{\text {oom }}\right)^{2}-\left(\tau_{0}^{\text {oop }}\right)^{2}}{4 s_{z} \tau_{0}}, \\
S_{z z} & =\frac{-\left(\tau_{0}^{\text {oop }}\right)^{2}-\left(\tau_{0}^{\text {oom }}\right)^{2}+2 \tau_{0}^{2}}{2 s_{z}^{2} \tau_{0}}+\frac{p_{z}^{2}}{\tau_{0}}, \\
S_{y z} & =\frac{-\left(\tau_{0}^{o p p}\right)^{2}-\left(\tau_{0}^{\text {omm }}\right)^{2}+\left(\tau_{0}^{\text {opm }}\right)^{2}+\left(\tau_{0}^{\text {omp }}\right)^{2}}{8 s_{y} s_{z} \tau_{0}}+\frac{p_{y} p_{z}}{\tau_{0}}, \\
S_{z x} & =\frac{-\left(\tau_{0}^{\text {pop }}\right)^{2}-\left(\tau_{0}^{\text {mom }}\right)^{2}+\left(\tau_{0}^{\text {oom }}\right)^{2}+\left(\tau_{0}^{m o p}\right)^{2}}{8 s_{z} s_{x} \tau_{0}}+\frac{p_{z} p_{x}}{\tau_{0}}, \\
N_{z z} & =\frac{\left(\tau_{\text {oom }}^{\text {oop }}\right)^{2}+\left(\tau_{\text {oop }}^{\text {oom }}\right)^{2}-\left(\tau_{\text {oop }}^{\text {oop }}\right)^{2}-\left(\tau_{\text {oom }}^{\text {oom }}\right)^{2}}{8 s_{z} g_{z} \tau_{0}}-\frac{p_{z} q_{z}}{\tau_{0}}, \\
N_{z y} & =\frac{\left(\tau_{\text {omo }}^{\text {oop }}\right)^{2}+\left(\tau_{\text {opo }}^{\text {oom }}\right)^{2}-\left(\tau_{\text {opo }}^{\text {oop }}\right)^{2}-\left(\tau_{\text {omo }}^{\text {oom }}\right)^{2}}{8 s_{z} g_{y} \tau_{0}}-\frac{p_{z} q_{y}}{\tau_{0}} \\
N_{z x} & =\frac{\left(\tau_{\text {moo }}^{\text {oop }}\right)^{2}+\left(\tau_{\text {poo }}^{\text {oom }}\right)^{2}-\left(\tau_{\text {poo }}^{\text {oop }}\right)^{2}-\left(\tau_{\text {moo }}^{\text {oom }}\right)^{2}}{8 s_{z} g_{x} \tau_{0}}-\frac{p_{z} q_{x}}{\tau_{0}}
\end{aligned}
$$




\section{Appendix E}

\section{A Reflector Independent Formulation of the Weight Functions}

The true-amplitude weight functions from Chapter 6 can be expressed in a manner which is independent of a priori knowledge about the reflector. This is possible since the weight functions were derived for the stationary point, i.e. the actually reflected ray. For this specific ray terms which previously required knowledge of the reflector orientation can be expressed using Snell's law. In this appendix I derive this new, reflector independent formulation of the weight functions.

The general weight function valid for PP and PS data which was derived in Chapter 6 reads

$$
W\left(\boldsymbol{\xi}^{*}, M\right)=\frac{1}{V_{s}} \sqrt{\cos \vartheta_{s} \cos \vartheta_{g}} \sqrt{\frac{\cos \theta_{1}}{\cos \theta_{2}}} \frac{\left|\operatorname{det}\left(\underline{\overline{\mathbf{N}}}_{1}^{\top} \underline{\underline{\Sigma}}+\underline{\overline{\mathbf{N}}}_{2}^{\top} \underline{\Sigma}\right)\right|}{\sqrt{\left|\operatorname{det} \underline{\bar{N}}_{1} \operatorname{det} \underline{\bar{N}}_{2}\right|}} e^{-i \frac{\pi}{2}\left(\kappa_{1}+\kappa_{2}\right)} .
$$

As a reminder, the angles $\vartheta_{I}$ are the emergence and incidence angles on the registration surface at the source $(I=s)$ and the receiver $(I=g)$. The angles $\theta_{I}$ are the incidence angle $(I=1)$ at the reflector and the reflection angle $(I=2)$. The $2 \times 2$ matrices $\underline{\mathbf{N}}_{I}$ result from a rotation of the $3 \times 3$ matrices $\underline{\hat{\mathrm{N}}}_{I}$ into the tangent plane of the reflector (denoted by the bar $^{-}$). This rotation can only be carried out if the orientation of the reflector with respect to the global Cartesian system is known in which the matrices $\underline{\hat{N}}_{I}$ are defined.

In the following I will show how the weight function (E.0.1) can be written in terms of of the matrices $\underline{\mathrm{N}}_{I}$ and the corresponding slowness vectors without needing to know the reflector orientation. The difference between the matrices $\underline{\mathrm{N}}_{I}$ and $\underline{\mathrm{N}}_{I}$ is that the $\underline{\mathrm{N}}_{I}$ are defined in the interface coordinate system associated with the reflector, whereas the matrices $\underline{\mathrm{N}}_{I}$ are the upper left submatrices of the mixed derivative matrices $\underline{\hat{N}}_{I}$ in the global Cartesian coordinate system.

From Equation (5.2.15) I use the relationship between $\underline{\mathrm{N}}$ and the matrix $\underline{\mathrm{Q}}_{2}$ between two points (denoted by $a$ and $b$, where index $a$ is either $s$ or $g$ in the registration surface while index $b$ is 1 or 2 at the interface):

$$
\underline{\mathrm{Q}}_{2}^{-1}=\underline{\mathrm{H}}_{a}^{-\top} \underline{\mathrm{N}} \underline{\mathrm{H}}_{b}^{-1} .
$$


A similar relationship exists between $\underline{Q}_{2}$ and $\underline{\bar{N}}$ (Hubral et al., 1992a):

$$
\underline{\overline{\mathrm{N}}}=\underline{\mathrm{G}}_{a}^{\top} \underline{\mathrm{Q}}_{2}^{-1} \underline{\mathrm{G}}_{b}
$$

where the matrices $\underline{G}_{I}$ are the upper left $2 \times 2$ submatrices of the transformation matrices between the interface and ray centred coordinates (see Section 2.8). Combining Equations (E.0.2) and (E.0.3) leads to

$$
\underline{\overline{\mathrm{N}}}=\underline{\mathrm{G}}_{a}^{\top} \underline{\mathrm{H}}_{a}^{-\top} \underline{\mathrm{N}} \underline{\mathrm{H}}_{b}^{-1} \underline{\mathrm{G}}_{b}
$$

In the registration surface I assume that the global and interface coordinate systems coincide. In this case the matrices $\underline{\mathrm{G}}_{a}$ and $\underline{\mathrm{H}}_{a}$ are equal, thus $\underline{\mathrm{G}}_{a}^{\top} \underline{\mathrm{H}}_{a}^{-\top}=\underline{1}$ at both the source and the receiver position. At the image point so far neither $\underline{\mathrm{G}}_{b}$ nor $\underline{\mathrm{H}}_{b}$ are determined since the orientation of the interface is unknown, as are the base vectors $\overrightarrow{\boldsymbol{e}}_{i}$ of the ray centred coordinate system. However, under the assumption that the two rays associated with the indices 1 and 2 correspond to a reflection, i.e. the stationary ray, I define the ray centred coordinate base vectors as illustrated in Figure E.1. The vectors $\overrightarrow{\boldsymbol{\imath}}_{i}$ are the base vectors of the interface coordinate system, the $\overrightarrow{\boldsymbol{e}}_{i}^{(I)}$ are those of the ray centred coordinate systems for the rays denoted by $I=1,2$ in the weight function. The vectors $\overrightarrow{\boldsymbol{e}}_{3}^{(I)}$ are determined by the slownesses vectors. Since

$$
\overrightarrow{\boldsymbol{e}}_{2}=\overrightarrow{\boldsymbol{e}}_{2}^{(1)}=\overrightarrow{\boldsymbol{e}}_{2}^{(2)}=\overrightarrow{\boldsymbol{\imath}}_{2}
$$

is defined to be perpendicular to the plane of propagation (see Figure E.1), it can be computed from the vector product of $\overrightarrow{\boldsymbol{e}}_{3}^{(1)}$ and $\overrightarrow{\boldsymbol{e}}_{3}^{(2)}$, which yields a vector perpendicular to both $\overrightarrow{\boldsymbol{e}}_{3}^{(I)}$. Therefore $\overrightarrow{\boldsymbol{e}}_{2}$ is given by

$$
\overrightarrow{\boldsymbol{e}}_{2}=\frac{1}{\sin \chi} \overrightarrow{\boldsymbol{e}}_{3}^{(1)} \times \overrightarrow{\boldsymbol{e}}_{3}^{(2)}
$$

where the factor $\sin ^{-1} \chi$ ensures that $\overrightarrow{\boldsymbol{e}}_{2}$ is a unit vector. Using the fact that the unit vectors $\overrightarrow{\boldsymbol{e}}_{i}$ form a right-handed orthonormal system, it follows with $\left|\overrightarrow{\boldsymbol{e}}_{3}^{(1)} \cdot \overrightarrow{\boldsymbol{e}}_{3}^{(2)}\right|=\cos \chi$ that

$$
\begin{aligned}
& \overrightarrow{\boldsymbol{e}}_{1}^{(1)}=\overrightarrow{\boldsymbol{e}}_{2} \times \overrightarrow{\boldsymbol{e}}_{3}^{(1)}=\frac{1}{\sin \chi}\left(\overrightarrow{\boldsymbol{e}}_{3}^{(1)} \times \overrightarrow{\boldsymbol{e}}_{3}^{(2)}\right) \times \overrightarrow{\boldsymbol{e}}_{3}^{(1)}=\frac{1}{\sin \chi}\left(\overrightarrow{\boldsymbol{e}}_{3}^{(2)}-\overrightarrow{\boldsymbol{e}}_{3}^{(1)} \cos \chi\right) \\
& \overrightarrow{\boldsymbol{e}}_{1}^{(2)}=\overrightarrow{\boldsymbol{e}}_{2} \times \overrightarrow{\boldsymbol{e}}_{3}^{(2)}=\frac{1}{\sin \chi}\left(\overrightarrow{\boldsymbol{e}}_{3}^{(1)} \times \overrightarrow{\boldsymbol{e}}_{3}^{(2)}\right) \times \overrightarrow{\boldsymbol{e}}_{3}^{(2)}=\frac{1}{\sin \chi}\left(-\overrightarrow{\boldsymbol{e}}_{3}^{(1)}+\overrightarrow{\boldsymbol{e}}_{3}^{(2)} \cos \chi\right) .
\end{aligned}
$$

With (see (5.2.7))

$$
\overrightarrow{\boldsymbol{e}}_{3}^{(I)}=\frac{1}{V} \boldsymbol{q}_{0}^{(I)}=\left(\begin{array}{c}
-\sin \vartheta_{I} \cos \varphi_{I} \\
\sin \vartheta_{I} \sin \varphi_{I} \\
\cos \vartheta_{I}
\end{array}\right)
$$




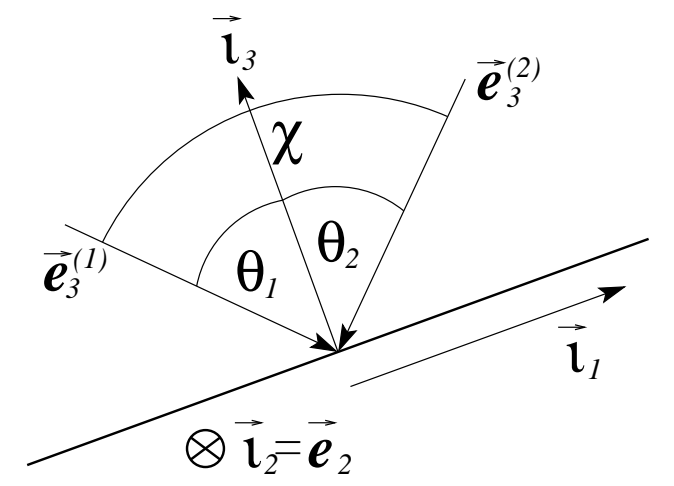

Figure E.1: Definition of the ray centred coordinate system associated with the reflected ray: $\overrightarrow{\boldsymbol{\imath}}_{3}$ is normal to the interface, $\overrightarrow{\boldsymbol{\imath}}_{1}$ lies in the propagation plane as well as in the tangent plane of the interface, and $\overrightarrow{\boldsymbol{\imath}}_{2}$ is given by $\overrightarrow{\boldsymbol{\imath}}_{3} \times \overrightarrow{\boldsymbol{\imath}}_{1}$. The base vectors of the ray centred systems, $\overrightarrow{\boldsymbol{e}}_{i}^{(I)}$ are defined such that $\overrightarrow{\boldsymbol{e}}_{2}^{(I)}=\overrightarrow{\boldsymbol{\imath}}_{2}$. The vectors $\overrightarrow{\boldsymbol{e}}_{3}^{(I)}=\overrightarrow{\boldsymbol{t}}^{(I)}$ are tangent to the rays. They are given by the directions of the slowness vectors. The angles $\theta_{I}$ are the incidence angles on the reflector, and the angle $\chi=\theta_{1}+\theta_{2}$.

the matrices $\underline{\mathrm{H}}_{I}^{-1}$ are

$$
\begin{aligned}
& \underline{\mathrm{H}}_{1}^{-1}=\frac{1}{\cos \vartheta_{1} \sin \chi} \times \\
& \left(\begin{array}{cc}
\sin \vartheta_{1} \cos \varphi_{1} \cos \vartheta_{2}-\sin \vartheta_{2} \cos \varphi_{2} \cos \vartheta_{1} & \sin \vartheta_{2} \sin \varphi_{2} \cos \vartheta_{1}-\sin \vartheta_{1} \sin \varphi_{1} \cos \vartheta_{2} \\
\sin \vartheta_{1} \sin \varphi_{1} \cos \chi-\sin \vartheta_{2} \sin \varphi_{2} & \sin \vartheta_{1} \cos \varphi_{1} \cos \chi-\sin \vartheta_{2} \cos \varphi_{2}
\end{array}\right) \\
& \underline{\mathrm{H}}_{2}^{-1}=\frac{1}{\cos \vartheta_{2} \sin \chi} \times \\
& \left(\begin{array}{cc}
\sin \vartheta_{1} \cos \varphi_{1} \cos \vartheta_{2}-\sin \vartheta_{2} \cos \varphi_{2} \cos \vartheta_{1} & \sin \vartheta_{2} \sin \varphi_{2} \cos \vartheta_{1}-\sin \vartheta_{1} \sin \varphi_{1} \cos \vartheta_{2} \\
-\sin \vartheta_{2} \sin \varphi_{2} \cos \chi+\sin \vartheta_{1} \sin \varphi_{1} & -\sin \vartheta_{2} \cos \varphi_{2} \cos \chi+\sin \vartheta_{1} \cos \varphi_{1}
\end{array}\right) .
\end{aligned}
$$

The matrices $\underline{\underline{G}}_{I}$ are given by (see Section 2.8 )

$$
G_{i j}=\overrightarrow{\boldsymbol{\imath}}_{i} \cdot \overrightarrow{\boldsymbol{e}}_{j}
$$

This leads to (see also Figure E.1)

$$
\underline{\mathrm{G}}_{1}=\underline{\mathrm{G}}_{1}^{\top}=\left(\begin{array}{cc}
-\cos \theta_{1} & 0 \\
0 & 1
\end{array}\right) \quad \text { and } \quad \underline{\mathrm{G}}_{2}=\underline{\mathrm{G}}_{2}^{\top}=\left(\begin{array}{cc}
-\cos \theta_{2} & 0 \\
0 & 1
\end{array}\right)
$$

The angles $\theta_{I}$ are the incidence angles on the interface. They cannot be computed without knowing the orientation of the interface. However, it is not necessary to know the angles $\theta_{I}$ themselves as I will now demonstrate. Inserting Equation (E.0.4) into the weight function 
(E.0.1) leads to

$$
\begin{aligned}
\frac{\left|\operatorname{det}\left(\underline{\overline{\mathrm{N}}}_{1}^{\top} \underline{\Sigma}+\underline{\overline{\mathrm{N}}}_{2}^{\top} \underline{\Gamma}\right)\right|}{\sqrt{\left|\operatorname{det} \underline{\bar{N}}_{1} \operatorname{det} \underline{\mathrm{N}}_{2}\right|}} & =\frac{\left|\operatorname{det}\left(\underline{\mathrm{G}}_{1}^{\top} \underline{\mathrm{H}}_{1}^{-\top} \underline{\mathrm{N}}_{1}^{\top} \underline{\Sigma}+\underline{\mathrm{G}}_{2}^{\top} \underline{\mathrm{H}}_{2}^{-\top} \underline{\mathrm{N}}_{2}^{\top} \underline{\Gamma}\right)\right|}{\sqrt{\left|\operatorname{det}\left(\underline{\mathrm{G}}_{1}^{\top} \underline{\mathrm{H}}_{1}^{-\top} \underline{\mathrm{N}}_{1}\right) \operatorname{det}\left(\underline{\mathrm{G}}_{2}^{\top} \underline{\mathrm{H}}_{2}^{-\top} \underline{\mathrm{N}}_{2}\right)\right|}} \\
& =\sqrt{\cos \vartheta_{1} \cos \vartheta_{2}} \sqrt{\frac{\cos \theta_{1}}{\cos \theta_{2}}} \frac{\left|\operatorname{det}\left(\underline{\mathrm{H}}_{1}^{-\top} \underline{\mathrm{N}}_{1}^{\top} \underline{\Sigma}+\underline{\mathrm{G}}_{1}^{-1} \underline{\mathrm{G}}_{2} \underline{\mathrm{H}}_{2}^{-\top} \underline{\mathrm{N}}_{2}^{\top} \underline{\Gamma}\right)\right|}{\sqrt{\left|\operatorname{det} \underline{\mathrm{N}}_{1} \operatorname{det} \underline{\mathrm{N}}_{2}\right|}},
\end{aligned}
$$

where $\left|\operatorname{det} \underline{H}_{I}\right|=\cos \vartheta_{I}$ and $\left|\operatorname{det} \underline{G}_{I}\right|=\cos \theta_{I}$ was used. With

$$
\underline{\mathrm{G}}_{I}^{-1}=\left(\begin{array}{cc}
-\frac{1}{\cos \theta_{I}} & 0 \\
0 & 1
\end{array}\right)
$$

the matrix product $\underline{G}_{1}^{-1} \underline{G}_{2}$ in (E.0.12) becomes

$$
\underline{\mathrm{G}}_{1}^{-1} \underline{\mathrm{G}}_{2}=\left(\begin{array}{cc}
-\frac{1}{\cos \theta_{1}} & 0 \\
0 & 1
\end{array}\right)\left(\begin{array}{cc}
-\cos \theta_{2} & 0 \\
0 & 1
\end{array}\right)=\left(\begin{array}{cc}
\frac{\cos \theta_{2}}{\cos \theta_{1}} & 0 \\
0 & 1
\end{array}\right) \text {. }
$$

This means that the angles $\theta_{I}$ themselves do not determine the weight function (E.0.1) but the ratio of their cosines does. Since for monotypic waves $\theta_{1}=\theta_{2}$, the new weight function in terms of matrices $\underline{\mathrm{N}}_{I}$ instead of $\underline{\bar{N}}_{I}$ reads

$$
W\left(\boldsymbol{\xi}^{*}, M\right)=\frac{1}{V_{s}} \sqrt{\cos \vartheta_{s} \cos \vartheta_{g}} \sqrt{\cos \vartheta_{1} \cos \vartheta_{2}} \frac{\left|\operatorname{det}\left(\underline{\mathrm{H}}_{1}^{-\top} \underline{\mathbf{N}}_{1}^{\top} \underline{\Sigma}+\underline{\mathrm{H}}_{2}^{-\top} \underline{\mathrm{N}}_{2}^{\top} \underline{\Gamma}\right)\right|}{\sqrt{\left|\operatorname{det} \underline{\mathrm{N}}_{1} \operatorname{det} \underline{\mathrm{N}}_{2}\right|}} e^{-i \frac{\pi}{2}\left(\kappa_{1}+\kappa_{2}\right)}
$$

To obtain a weight function suitable for migration of converted wave data, an expression for the ratio of the cosines of the angles $\theta_{I}$ is required which does not depend on a priori information about the reflector. This expression can be found with the transformation matrix $\underline{\text { Z }}$ between global and interface Cartesian coordinates

$$
\underline{\hat{\mathrm{Z}}}=\underline{\hat{\mathrm{H}}}_{1} \underline{\hat{\mathrm{G}}}_{1}^{-1}=\underline{\hat{\mathrm{H}}}_{2} \underline{\hat{\mathrm{G}}}_{2}^{-1}
$$

or, in index notation

$$
Z_{i k}=H_{i j}^{(1)} G_{k j}^{(1)}=H_{i j}^{(2)} G_{k j}^{(2)}
$$

where $\underline{\hat{\mathrm{G}}}^{-1}=\underline{\hat{\mathrm{G}}}^{\top}$ was used. With Snell's law, stating that

$$
p=\frac{\sin \theta_{1}}{V_{1}}=\frac{\sin \theta_{2}}{V_{2}}
$$

Equation (E.0.17) leads to the following system of equations:

$$
\begin{aligned}
Z_{i 3} & =-H_{i 3}^{(1)} \cos \theta_{1}+H_{i 1}^{(1)} \sin \theta_{1}=-H_{i 3}^{(2)} \cos \theta_{2}+H_{i 1}^{(2)} \sin \theta_{2} \\
& =-H_{i 3}^{(1)} \cos \theta_{1}+H_{i 1}^{(1)} V_{1} p=-H_{i 3}^{(2)} \cos \theta_{2}+H_{i 1}^{(2)} V_{2} p .
\end{aligned}
$$


Substituting the elements of $\underline{\hat{H}}_{I}$ into the system (E.0.19) and elimination of $p$ yields after some algebra

$$
\frac{\cos \theta_{1}}{\cos \theta_{2}}=\frac{V_{1} \cos \chi+V_{2}}{V_{2} \cos \chi+V_{1}}
$$

With this result the new weight function for the migration of converted waves is given by $W\left(\boldsymbol{\xi}^{*}, M\right)=\frac{1}{V_{s}} \sqrt{\cos \vartheta_{s} \cos \vartheta_{g}} \frac{V_{1} \cos \chi+V_{2}}{V_{2} \cos \chi+V_{1}} \frac{\left|\operatorname{det}\left(\underline{\mathrm{H}}_{1}^{-\top} \underline{\mathrm{N}}_{1}^{\top} \underline{\Sigma}+\underline{\mathrm{G}}_{1}^{-1} \underline{\mathrm{G}}_{2} \underline{\mathrm{H}}_{2}^{-\top} \underline{\mathrm{N}}_{2}^{\top} \underline{\Gamma}\right)\right|}{\sqrt{\left|\operatorname{det} \underline{\mathrm{N}}_{1} \operatorname{det} \underline{\mathrm{N}}_{2}\right|}} e^{-i \frac{\pi}{2}\left(\kappa_{1}+\kappa_{2}\right)}$

with

$$
\underline{\mathrm{G}}_{1}^{-1} \underline{\mathrm{G}}_{2}=\left(\begin{array}{cc}
\frac{V_{2} \cos \chi+V_{1}}{V_{1} \cos \chi+V_{2}} & 0 \\
0 & 1
\end{array}\right)
$$

For a 2.5-D symmetry, the situation simplifies as the non-diagonal elements of the matrices $\underline{\mathrm{H}}_{I}$ become zero (the angles $\varphi_{I}$ are either $0^{\circ}$ or $180^{\circ}$ ). Also, in $2-\mathrm{D}$ and $2.5-\mathrm{D}$ $\chi=\theta_{1}+\theta_{2}=\vartheta_{1}+\vartheta_{2}$. In 2-D, the matrices reduce to scalars with

$$
\begin{aligned}
G_{1} & =-\cos \theta_{1} \\
G_{2} & =-\cos \theta_{2} \\
\left|H_{1}\right| & =\cos \vartheta_{1} \\
\left|H_{2}\right| & =\cos \vartheta_{2}
\end{aligned}
$$

where the sign of $H_{I}$ depends on that of the $x$ component of the slowness vector. 


\section{Bibliography}

Bancroft, J. C., 1998, A practical understanding of pre- and poststack migrations, volume 2 (prestack): SEG, Tulsa.

Beylkin, G., 1985, Imaging of discontinuities in the inverse scattering problem by inversion of a causal generalized Radon transform: Journal of Mathematical Physics, 26, 99-108.

Bleistein, N., 1984, Mathematical methods for wave phenomena: Academic Press.

Bleistein, N., 1986, Two-and-one-half dimensional in-plane wave propagation: Geophysical Prospecting, 34, 686-703.

Bleistein, N., 1987, On the imaging of reflectors in the earth: Geophysics, 52, 931-942.

Bortfeld, R., 1982, Phänomene und Probleme beim Modellieren und Invertieren, in Modellverfahren bei der Interpretation seismischer Daten: DVGI, Fachausschuß Geophysik.

Bortfeld, R., 1989, Geometrical ray theory: rays and traveltimes in seismic systems (second-order approximations of the traveltimes): Geophysics, 54, 342-349.

Brandsberg-Dahl, S., deHoop, M. V., and Ursin, B., 2001, AVA analysis and compensation on common image gathers in the angle domain: 63th Ann. Internat. Mtg., Eur. Assn. Expl. Geophys., Expanded Abstracts.

Brokešová, J., Dekker, S., and Duijndam, A., 1994, Applicability of high-frequency asymptotic methods for the model PICROCOL: 64th Ann. Internat. Mtg., Soc. Expl. Geophys., Expanded Abstracts, 783-786.

Brokešová, J., 1996, Construction of ray synthetic seismograms using interpolation of traveltimes and ray amplitudes: Pure and Applied Geophysics, 148, 481-502.

Buske, S., 2000, Finite difference solution of the ray theoretical transport equation: Ph.D. thesis, Universität Frankfurt.

Červený, V., and deCastro, M. A., 1993, Application of dynamic ray tracing in the 3-D inversion of seismic reflection data: Geophysical Journal International, 113, 776-779.

Červený, V., and Hron, F., 1980, The ray series method and dynamic ray tracing systems for 3D inhomogeneous media: Bulletin of the Seismological Society of America, 70, $47-77$. 
Červený, V., and Pšenčík, I., 1972, Rays and travel-time curves in inhomogeneous anisotropic media: Journal of Geophysics, 38, 565-577.

Červený, V., and Pšenčík, I., 1984, Numerical modelling of seismic wave fields in 2-D laterally varying layered structures by the ray method in Engdahl, E., Ed., Documentation of Earthquake Algorithms: World Data Center for Solid Earth Geophysicists, Rep. SE-35, 36-40.

Červený, V., and Ravindra, R., 1971, Theory of seismic head waves: University of Toronto Press.

Cervený, V., and Soares, J. E. P., 1992, Fresnel volume ray tracing: Geophysics, 57, 902-911.

Červený, V., Molotkov, I. A., and Pšenčík, I., 1977, Ray method in seismology: Univerzita Karlova, Praha.

Červený, V., 1972, Seismic rays and ray intensities in inhomogeneous anisotropic media: Geophysical Journal of the Royal astronomical Society, 29, 1-13.

Červený, V., 1985, The application of ray tracing to the numerical modeling of seismic wavefields in complex structures, in Dohr, G., Ed., Seismic shear waves, Part A: Theory: Geophysical Press, London - Amsterdam, 1-124.

Červený, V., 2001, Seismic ray theory: Cambridge University Press.

Coman, R., and Gajewski, D., 2001, Estimation of multivalued arrivals in 3-D models using wavefront ray tracing: 71th Ann. Internat. Mtg., Soc. Expl. Geophys., Expanded Abstracts.

Courant, R., and Hilbert, D., 1962, Methods of mathematical physics, vol.2: Partial differential equations: Interscience, New York.

Daley, P. F., and Hron, F., 1979a, Reflection and transmission coefficients for seismic waves in ellipsoidally anisotropic media: Geophysics, 44, 27-38.

Daley, P. F., and Hron, F., 1979b, SH waves in layered transversely isotropic media - an asymptotic expansion approach: Bulletin of the Seismological Society of America, 69, 689-711.

deBazelaire, E., 1988, Normal moveout revisited - inhomogeneous media and curved interfaces: Geophysics, 53, 143-157.

deHoop, M., and Bleistein, N., 1997, Generalized Radon transform inversions for reflectivity in anisotropic elastic media: Inverse Problems, 13, 669-690.

deHoop, M., Spencer, C., and Burridge, R., 1999, The resolving power of seismic amplitude data: an anisotropic inversion/migration approach: Geophysics, 64, 852-873. 
Dellinger, J. A., Gray, S. H., Murphy, G. E., and Etgen, J. T., 2000, Efficient 2.5-D true-amplitude migration: Geophysics, 65, 943-950.

Ettrich, N., and Gajewski, D., 1996, Wave front construction in smooth media for prestack depth migration: Pure and Applied Geophysics, 148, 481-502.

Gajewski, D., and Pšenčík, I., 1987, Computation of high frequency wavefields in 3-D laterally inhomogeneous anisotropic media: Geophysical Journal of the Royal Astronomical Society, 91, 383-411.

Gajewski, D., and Pšenčík, I., 1990, Vertical seismic profile synthetics by dynamic ray tracing in laterally varying layered anisotropic structures: Journal of Geophysical Research, 95, 11,301-11,315.

Gajewski, D., and Vanelle, C., 2001, Extending the $T^{2}-X^{2}$ method to 3-D heterogeneous media: 71th Ann. Internat. Mtg., Soc. Expl. Geophys., Expanded Abstracts.

Gajewski, D., 1993, Radiation from point sources in general anisotropic media: Geophysical Journal International, 113, 299-317.

Gajewski, D., 1998, Determining the ray propagator from traveltimes: 68th Ann. Internat. Mtg., Soc. Expl. Geophys., Expanded Abstracts, 1900-1903.

Gardner, G. H. F., French, W. S., and Matzuk, T., 1974, Elements of migration and velocity analysis: Geophysics, 39, 811-825.

Gelchinsky, B., Berkovitch, A., and Keydar, S., 1999, Multifocusing homeomorphic imaging. Part 1: Basic concepts and formulas: Journal of Applied Geophysics, 42, 229-242.

Gerea, C., Nicoletis, L., and Granger, P.-Y., 2000, Multi component true amplitude anisotropic imaging: Anisotropy 2000: Fractures, converted waves and case studies (9. International Workshop on Seismic Anisotropy), Soc. Expl. Geophys., Expanded Abstracts.

Gjøystdal, H., Reinhardsen, J. E., and Ursin, B., 1984, Traveltime and wavefront curvature calculations in three-dimensional inhomogeneous layered media with curved interfaces: Geophysics, 49, 1466-1494.

Hanitzsch, C., Schleicher, J., and Hubral, P., 1994, True-amplitude migration of 2D synthetic data: Geophysical Prospecting, 42, 445-462.

Hanitzsch, C., Jin, S., Tura, A., and Audebert, F., 2001, Efficient amplitude-preserved prestack depth migration: 63th Ann. Internat. Mtg., Eur. Assn. Expl. Geophys., Expanded Abstracts, A-25.

Hanitzsch, C., 1995, Amplitude preserving prestack depth migration/inversion in laterally inhomogeneous media: Ph.D. thesis, Universität Karlsruhe.

Hanitzsch, C., 1997, Comparison of weights in prestack amplitude-preserving depth migration: Geophysics, 62, 1812-1816. 
Helbig, K., 1983, Elliptical anisotropy - its significance and meaning: Geophysics, 48, $825-832$.

Hubral, P., and Krey, T., 1980, Interval velocities from seismic reflection time measurements: SEG, Tulsa.

Hubral, P., Schleicher, J., and Tygel, M., 1992a, Three-dimensional paraxial ray properties, Part 1: Basic relations: Journal of Seismic Exploration, 1, 265-279.

Hubral, P., Schleicher, J., and Tygel, M., 1992b, Three-dimensional paraxial ray properties, Part 2: Applications: Journal of Seismic Exploration, 1, 347-362.

Hubral, P., Schleicher, J., and Tygel, M., 1993a, Three dimensional primary zero offset reflections: Geophysics, 58, 692-702.

Hubral, P., Schleicher, J., Tygel, M., and Hanitzsch, C., 1993b, Determination of Fresnel zones from traveltime measurements: Geophysics, 58, 703-712.

Hubral, P., 1983, Computing true amplitude reflections in a laterally inhomogeneous earth: Geophysics, 48, 1051-1062.

Jäger, R., Mann, J., Höcht, G., and Hubral, P., 2001, Common-reflection-surface stack: Image and attributes: Geophysics, 66, 97-109.

Jech, J., and Pšenčík, I., 1989, First order perturbation method for anisotropic media: Geophysical Journal International, 99, 369-376.

Kabir, N., and Verschuur, E., 1995, Reconstruction of missing offsets by parabolic Radon transform: Geophysical Prospecting, 43, 347-368.

Keho, T. H., and Beydoun, W. B., 1988, Paraxial Kirchhoff migration: Geophysics, 53, 1540-1546.

Leidenfrost, A., Kosloff, D., and Gajewski, D., 1998, A 3D FD eikonal solver for noncubical grids: 60th Ann. Internat. Mtg., Eur. Assn. Expl. Geophys., Expanded Abstracts.

Leidenfrost, A., Ettrich, N., Gajewski, D., and Kosloff, D., 1999, Comparison of six different methods for calculating traveltimes: Geophysical Prospecting, 47, 269-297.

Leidenfrost, A., 1998, Fast computation of traveltimes in two and three dimensions: Ph.D. thesis, University of Hamburg.

Martins, J. L., Schleicher, J., Tygel, M., and Santos, L., 1997, 2.5-D true-amplitude migration and demigration: Journal of Seismic Exploration, 6, 159-180.

Mendes, M., 2000, Green's function interpolation for prestack imaging: Geophysical Prospecting, 48, 49-62. 
Menyoli, E. M., and Gajewski, D., 2001, Kirchhoff migration of converted waves applying perturbation method for computing Greens functions: 63th Ann. Internat. Mtg., Eur. Assn. Expl. Geophys., Expanded Abstracts.

Peles, O., Kosloff, D., Koren, Z., and Tygel, M., 2001, A practical approach to amplitude preserving migration: WIT Workshop 2001 on Seismic True-Amplitude Reflections.

Popov, M. M., and Pšenčík, I., 1978, Ray amplitudes in inhomogeneous media with curved interfaces: Geofysikální Sborník, 24, 111-129.

Pusey, L. C., and Vidale, J. E., 1991, Accurate finite-difference calculation of WKBJ traveltimes and amplitudes: 61st Ann. Internat. Mtg., Soc. Expl. Geophys., Expanded Abstracts, 1513-1516.

Pšenčík, I., and Teles, T. N., 1996, Point source radiation in inhomogeneous anisotropic structures: Pure and Applied Geophysics, 148, 591-623.

Pšenčík, I., 1994, Introduction to seismic methods: Lecture notes, PPPG/UFBa.

Schleicher, J., Tygel, M., and Hubral, P., 1993a, 3D true-amplitude finite-offset migration: Geophysics, 58, 1112-1126.

Schleicher, J., Tygel, M., and Hubral, P., 1993b, Parabolic and hyperbolic paraxial twopoint traveltimes in 3D media: Geophysics, 41, 495-513.

Schleicher, J., Hubral, P., Tygel, M., and Jaya, M. S., 1997, Minimum apertures and Fresnel zones in migration and demigration: Geophysics, 62, 183-194.

Schleicher, J., Tygel, M., Ursin, B., and Bleistein, N., 2001, The Kirchhoff-Helmholtz integral for anisotropic elastic media: Wave Motion, pages 353-364.

Schleicher, J., 1993, Bestimmung von Reflexionskoeffizienten aus Reflexionsseismogrammen: Ph.D. thesis, Universität Karlsruhe.

Spitz, S., 1991, Seismic trace interpolation: Geophysics, 50, 785-794.

Sun, J., 1999, On the aperture effect in 3D Kirchhoff-type migration: Geophysical Prospecting, 47, 1045-1076.

Taner, M. T., and Koehler, F., 1969, Velocity spectra - digital computer derivation and application of velocity functions: Geophysics, 34, 859-881.

Ursin, B., 1982, Quadratic wavefront and traveltime approximations in inhomogeneous layered media with curved interfaces: Geophysics, 47, 1012-1021.

Ursin, B., 1990, Offset dependent geometrical spreading in a layered medium: Geophysics, 55, 492-496.

Vanelle, C., and Gajewski, D., 1999, Determining geometrical spreading from traveltimes: 61th Ann. Internat. Mtg., Eur. Assn. Expl. Geophys., Expanded Abstracts, 1.56. 
Vanelle, C., and Gajewski, D., 2001a, Determining the optimum migration aperture from traveltimes: Journal of Seismic Exploration, 10, 205-224.

Vanelle, C., and Gajewski, D., 2001b, True-amplitude migration of PS converted waves: 71th Ann. Internat. Mtg., Soc. Expl. Geophys., Expanded Abstracts.

Vanelle, C., and Gajewski, D., 2002a, Second-order interpolation of traveltimes: Geophysical Prospecting, 50, 73-83.

Vanelle, C., and Gajewski, D., 2002b, True amplitude migration weights from traveltimes: Pure and Applied Geophysics (in press).

VanTrier, J., and Symes, W. W., 1990, Upwind finite-difference calculation of seismic traveltimes: 70th Ann. Internat. Mtg., Soc. Expl. Geophys., Expanded Abstracts, 10001003.

Vermeer, G. J. O., 1995, Discussion on "3-D true-amplitude finite-offset migration" by J. Schleicher, M. Tygel and P. Hubral (Geophysics, 58, 1112-1126) with reply by the authors: Geophysics, 60, 921-923.

Versteeg, R., and Grau, G., 1991, The Marmousi experience: 1990 EAEG workshop on Practical Aspects of Seismic Data Inversion, EAEG, Zeist, The Netherlands, Proceedings.

Vidale, J., and Houston, H., 1990, Rapid calculation of seismic amplitudes: Geophysics, 55, 1504-1507.

Vidale, J., 1990, Finite-difference calculation of traveltimes in three dimensions: Geophysics, 55, 521-526.

Vinje, V., Iversen, E., and Gjøystdal, H., 1993, Traveltime and amplitude estimation using wavefront construction: Geophysics, 58, 1157-1166.

Zhang, Y., Gray, S., and Young, J., 2000, Exact and approximate weights for Kirchhoff migration: 70th Ann. Internat. Mtg., Soc. Expl. Geophys., Expanded Abstracts, 10361039.

Zhang, Y., Bergler, S., and Hubral, P., 2001, Common-reflection-surface (CRS) stack for common offset: Geophysical Prospecting, 49, 709-718. 


\section{Acknowledgements}

\section{True Amplitudes...}

I am grateful to

- Prof. Dr. Dirk Gajewski for the opportunity to work with him and his invitation to share and teach me his enthusiasm in science. His encouragement and trust gave the fledgling scientist the courage to fly.

- Dr. Christian Hanitzsch for his encouragement and valuable comments on my work, and for his willingness to be co-reviewer of my Ph.D. thesis.

- Prof. Dr. Ulrich Bismayer, Dr. Christian Hübscher, and Prof. Dr. Wilfried Zahel for spending a share of their precious time on my examination committee.

- Dr. Richard Cook, Prof. Dr. Ivan Pšenčík, Prof. Dr. Jörg Schleicher, Prof. Dr. Martin Tygel, and several anonymous reviewers who not only improved my papers but also the work published in this thesis.

- Prof. Dr. Peter Hubral for his inspiring work and support.

- The past and present members of the Applied Geophysics Group Hamburg for continuous discussion, specifically to Radu Coman and Dr. Tim Bergmann for proof-reading, and to Dr. Ekkehart Tessmer for the miraculously well maintained computer network.

- Dipl.-Geophys. Alexander Goertz for a very helpful discussion.

- Christel Mynarik for invaluable support with the administration.

- Financial support from

- the European Commission (JOF3-CT97-0029),

- the German-Israeli-Foundation (I-524-018.08/97),

- the German Research Foundation (DFG, Ga 350-10),

- the sponsors of the Wave Inversion Technology (WIT) consortium.

\section{True Friendship...}

Many thanks to

- Björn, Michaela, Lukas, and Niels Hoffmann for reminding me of "real life" outside the ivory tower.

- Peter Hüttemann and Christoph Pohl for their company through the darkness towards the light.

- Dirk Möller for simply being there.

\section{True Love...}

One does not thank love. But I love you and thank you for accepting me as I am: Heide and Gérard, my parents, and Benedict, light of my life. 